UNIVERSITY OF BRASILIA

INSTITUTE OF PHYSICS

Generation and Recombination of Excited States in Organic Semiconductors

Luiz Antonio Ribeiro Junior

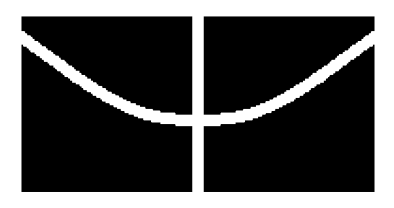

Brasilia, January of 2015 


\title{
Generation and Recombination of Excited States in Organic Semiconductors
}

\author{
By
}

\section{Luiz Antonio Ribeiro Junior}

Thesis submitted to the Institute of Physics at University of Brasilia as part of the requirements for the degree of doctor of physics.

Approved by:

Prof. Geraldo Magela e Silva

(Supervisor)

Prof. Paulo Eduardo de Brito (FUP/UnB)

Prof. Luiz Fernando Roncaratti Junior

$(\mathrm{IF} / \mathrm{UnB})$

Prof. Jonathan Fernando Teixeira

(IFB)

Prof. José Antonio Huamaní Coaquira $(\mathrm{IF} / \mathrm{UnB})$

Prof. Fernando Albuquerque de Oliveira

(Graduate Program Coordinator)

Brasilia, January 23 of 2015 
This thesis is dedicated to my wife, my children, my parents, and my brother. It is also dedicated in memory of my grandmother. 


\section{Acknowledgements}

I am deeply thankful to my parents Luiz Antonio and Zilda for guiding my steps until today with much love and wisdom. To my wife Barbara, source of love and encouragement, for always being by my side on all moments. To my children Lucas Gabriel, Luiz Eduardo, Luiza Beatriz, my brother Luiz Henrique and my cousin Danilo for making me a better person. To my uncles Fabio, Zenilda, Vagner, Eliane, Cleuber, and Joseane for all

the encouragement and affection. I cordially thank all my family (that includes my wife's family) for their affection and support.

I am sincerely thankful to my supervisor Prof. Geraldo M. e Silva for his, seemingly infinity, knowledge employed in the task of guiding me. To the researcher, co-supervisor, and also a good friend Prof. Wiliam F. da Cunha for having been a key and tireless collaborator in all the articles presented in this thesis, and I am sure that will be also in forthcoming works. To Profs. Ricardo Gargano, Pedro H. de Oliveira Neto, and Luiz Roncaratti by the help and encouragement. To Prof. Demétrio A. da Silva Filho for having built the bridge that has changed the course of my life. In particular, I would like to express my sincere thanks to my supervisor in the Linköping University, Prof. Sven Stafström (which has also a seemingly infinity knowledge), for the valuable opportunity to work under his insightful guidance. Not least, to my co-supervisor Prof. Mathieu Linares for his expertise and encouragement. Moreover, I am very thankful to Prof. Hukka Terttu of Tampere University of Technology for the promising and also fruitful collaboration.

I would also like to thank my great friends Luciano, Marcelo, Andrei, Makswel, Leander, Giovanni, Bruno, Rafael, Leonardo, and Bruno "Canudo" for the unconditional companionship. To the IF employees Sandra and Thales for all support. Finally, to the CAPES by the financial support. 
Abstract

Since the discovery of light emitting properties on phenyl-based organic semiconductors in the 90's, considerable efforts have been devoted to study the photophysical applications of conjugated polymers for development of new technologies in organic optoelectronic devices. The potential advantages in terms of ease of synthesis, flexibility, low cost, and large-area capability make the organic semiconductors attractive for the electronics industry, particularly when it comes to the promising development of a new display technology. There are many potential applications such as Organic Solar Cells, Thin-Film Transistors, and Organic Light Emitting Diodes (OLEDs). The recombination dynamics between quasi-particles, in order to generate excited species, has been identified as an important process in these applications. Furthermore, the products arising from the recombination mechanism as well as their final yields, have been shown to be directly related to the devices performance. One of the significant challenges in the science and technology of organic semiconductors is the characterization of the temperature and impurity effects on the generation and recombination of quasi-particles. Thus, understanding how such effects play the role of changing the formation dynamics of new products from the recombination process is of fundamental importance to the development of more efficient optoelectronic devices. In this thesis, the recombination dynamics between quasi-particles in organic semiconductors is numerically investigated under the influence of impurities, Coulomb interactions, temperature and an external electric field. The aim of this work is to give a physical picture of the products and their yields derived from the recombination of different kinds of quasi-particles in organic semiconductors, when the above-mentioned effects are considered and contribute to the understanding of these important processes, which may provide guidance to improve, for example, the electroluminescence yields in OLEDs. 


\section{Resumo}

Desde de a descoberta das propriedades de emissão de luz atribuídas aos semicondutores orgânicos baseados em grupos fenil na década de 90 , esforços consideráveis têm sido empregados no estudo de propriedades fotofísicas desses materiais. Vantagens interessantes como facilidade de síntese, flexibilidade, baixo custo de produção e vasta área de aplicação tornam os semicondutores orgânicos atrativos para a indústria de eletrônicos, particularmente quando a fabricação de uma nova tecnologia de telas é considerada. Existem várias aplicações em potencial tais como células solares orgânicas, transistores de filme-fino e diodos emissores de luz orgânicos (OLDEs). A dinâmica de recombinação entre quase-partículas, de maneira a formar espécies excitadas, tem sido identificada como um processo importante nessas aplicações. Um dos desafios relevantes na ciência e tecnologia de semicondutores orgânicos é a caracterização dos efeitos de temperatura e impureza sobre a geração e recombinação entre quase-partículas. Com isso, o entendimento de como tais efeitos desempenham o papel de alterar a dinâmica de formação de novos produtos é de fundamental importância para o desenvolvimento de dispositivos optoeletrônicos mais eficientes. Nesta tese, a dinâmica de recombinação entre quase-partículas em semicondutores orgânicos é investigada numericamente sob influência de impurezas, interações coulombianas, temperatura e campo elétrico externo. O objetivo desse trabalho é fornecer um panorama físico dos produtos e seus respectivos rendimentos resultantes da recombinação entre diferentes tipos de quase-partículas em semicondutores orgânicos, principalmente quando os efeitos mencionados acima são levados em consideração, contribuindo para a compreensão deste processo, que pode fornecer orientações para aprimorar, por exemplo, a eficiência de processos que envolvam eletroluminescência em dispositivos optoeletrônicos tais como OLEDs. 


\section{Contents}

1 Introduction $\quad 10$

1.1 Historical Background . . . . . . . . . . . . . . . . . . . . . . 10

1.2 The Polyacetylene . . . . . . . . . . . . . . . . . . . . . . . . 11

1.3 Applications . . . . . . . . . . . . . . . . . . . 13

1.3.1 Organic Light Emitting Diodes (OLEDs) . . . . . . . . . . 13

1.3.2 Organic Solar Cells (OSCs) . . . . . . . . . . . 15

1.4 The State of the Art . . . . . . . . . . . . . . . 16

1.5 Thesis Outline . . . . . . . . . . . . . . . . . . . . . . 21

2 Theoretical Background $\quad 27$

2.1 The Many-Body Problem . . . . . . . . . . . . . . . . . . 27

2.2 Antisymmetric Wavefunctions and the Slater determinant . . . . . . . 29

2.3 Second Quantization Formalism . . . . . . . . . . . . . . . . 32

2.4 The Tight-Binding Approach . . . . . . . . . . . . . . . . . . . . . . 35

2.5 The Langevin Equation . . . . . . . . . . . . . . . . . . . . . . . 38

3 The Su-Schrieffer-Heeger Model 41

3.1 The SSH-type Hamiltonian . . . . . . . . . . . . . . . . . . . . . . . . 41

3.2 The Dimerized Lattice . . . . . . . . . . . . . . . . . . . . . . . . 44

3.3 The Electric Field and the Symmetry Breaking Term . . . . . . . . . 47

3.4 Electron-Electron Interactions and Impurity Effects . . . . . . . . . . 48

3.5 The System Dynamics . . . . . . . . . . . . . . . . . . . . 48

3.6 Temperature Effects and Occupation Number . . . . . . . . . . 52

3.7 Charge Carriers and Excited States . . . . . . . . . . . . . . . . . 53

4 Conclusions and Perspectives $\quad 58$

$\begin{array}{ll}\text { Bibliography } & 65\end{array}$

$\begin{array}{ll}\text { A PAPER I } & 74\end{array}$

$\begin{array}{ll}\text { B PAPER II } & 80\end{array}$ 
$\begin{array}{llr}\text { C PAPER III } & 89\end{array}$

$\begin{array}{ll}\text { D PAPER IV } & 95\end{array}$

$\begin{array}{ll}\text { E PAPER V } & 103\end{array}$

$\begin{array}{llr}\text { F PAPER VI } & \mathbf{1 1 0}\end{array}$

G PAPER VII 122

$\begin{array}{ll}\text { H PAPER VIII } & 131\end{array}$

$\begin{array}{llr}\text { I PAPER IX } & 138\end{array}$

$\begin{array}{llr}\text { J PAPER X } & 148\end{array}$

$\begin{array}{lr}\text { K PAPER XI } & 154\end{array}$

$\begin{array}{llr}\text { L PAPER XII } & 163\end{array}$

$\begin{array}{lr}\text { M PAPER XIII } & 174\end{array}$ 


\section{List of Figures}

1.1 Schematic representation of three conjugated polymers: (a) transpolyacetylene, (b) cis-polyacetylene, and (c) poly ( $p$-phenylene vinylene). 12

1.2 A schematic representation of the working principle of an OLED. . . 14

1.3 A schematic representation of the working principle of an OSC. . . . 16

2.1 Molecular coordinate system for the many-body problem. . . . . . . . 28

3.1 Schematic representation of a dimerized trans-polyacetylene molecule with its displacement coordinate $u$, which denotes the unique degree of freedom. . . . . . . . . . . . . . . . . 42

3.2 Schematic representation of the electronic spectrum for a polyacetylene lattice containing (a) a positive polaron (hole-polaron) and (b) a negative polaron (electron-polaron). . . . . . . . . .

3.3 Schematic representation of the electronic spectrum for a polyacetylene lattice containing (a) a positive bipolaron (hole-bipolaron) and (b) a negative bipolaron (electron-bipolaron). . . . . . . . . . .

3.4 Schematic representation of the electronic spectrum for a polyacetylene lattice containing an exciton. . . . . . . . . . . 56 


\section{CHAPTER}

\section{Introduction}

In this chapter, a brief historical background regarding the discovery of conducting polymers is presented. An introduction about $\pi$-conjugated molecular systems, particularly polyacetylene, is provided. Furthermore, we briefly discuss the physical properties of some important applications to the recombination process between quasi-particles in organic-based optoelectronic devices. As a motivation, we present the state of the art referring to this issue from the framework of a tight-binding approach. Finally, the thesis outline is presented.

\subsection{Historical Background}

Since the discovery of conductivity in organic molecular crystals in the 60s, a huge amount of studies has been performed using this class of materials to figure out the mechanisms involved in the charge transport phenomena and in order to obtain new species of organic semiconductors [1-3]. Devoted to this research field, the group of Prof. Hideki Shirakawa was working on the synthesis of polymers from acetylene gas since the late 1960s [1]. During an experiment, in 1974, a PhD student under his guidance accidentally added thousands times more catalyst than it was usually required. As a result, it was obtained a silvery and shining film with elasticity similar to a plastic thin-sheet, named trans-polyacetylene [4-8].

Albeit the material obtained by Shirakawa and his student seemed a metallic-like film, they have found that it was not electrically conductive. In 1977, Shirakawa started a very fruitful collaboration with Alan Heeger and Alan MacDiarmid at the University of Pennsylvania that was aimed to obtain polyacetylene films with greater conductivity [9]. In the same year, they increased significantly the electrical conductivity of trans-polyacetylene by doping it with bromine [10]. Moreover, using well oriented samples of the resulting film, conductivity levels nearly as high as that to cooper were reached [11]. 
The results derived from the above-mentioned collaboration gave rise to a new generation of organic semiconductors, termed conjugated polymers. The interest about these materials increased dramatically since that decade. Thereafter, many other conjugated polymers have been both theoretically and experimentally studied. Particularly, after the discovery of the electroluminescence properties in $\operatorname{poly}(p-$ phenylene vinylene) films [12], conjugated polymers have shown a bright future as active materials in several technological applications such as Organic Light-Emitting Diodes (OLEDs) [13,14], Organic Solar Cells (OSCs) [15, 16], and Organic ThinFilm Transistors (OTFTs) [17,18]. Furthermore, the electronic and optical properties of conjugate polymers, together with their mechanical properties, processing advantages, versatility of chemical synthesis, and low cost make them attractive materials for the electronics industry, mainly when it comes to the promising development of a new display technology [19]. Due to the discoveries and advances made in the field of conducting polymers Heeger, MacDiarmid, and Shirakawa were awarded the Nobel Prize in Chemistry in the year 2000.

\subsection{The Polyacetylene}

The polyacetylene is one of the simplest conjugated polymers with an one-dimensional structure being formed from a long chain of carbon atoms [2,3]. The term conjugation represents the switching of double and single bonds between the carbon atoms that constitute the chain, where each carbon atom is bonded to a hydrogen atom and laterally connected to other two carbons in a linear arrangement $[2,3]$. During the 30 s and 40s, this polymer was subject of several controversial theoretical discussions regarding the role played by $\pi$-electrons. From a particular point of view, it was thought, by symmetry considerations, that if the polymer chain were conjugated, there would be a separation (gap) between the last occupied electronic state and the first empty one. Therefore, the material would have semiconducting properties. It was also thought that, if the electron cloud were delocalized throughout the system, the bond lengths would tend to posses the same size. Thus, the symmetry of the system would lead to a metallic-like band structure, i. e., with a solely half-filled band.

From the theoretical point of view, the controversy was solved understanding that, due to its polymeric structure, the polyacetylene would have one-dimensional properties, thus being subjected to the theorem enunciated in 1953 by Rudolph Peierls [20]: any one-dimensional conductor is unstable, subject to structural changes that 
may make it semiconducting. Therefore, this theorem was favorable to the point of view which pointed out that the conjugated polymer chain would be the more stable structure. In this kind of structure, the polyacetylene is weakly coupled to the neighboring chain; consequently, the resulting material is flexible. The bond conjugation produces two different distances between the $\mathrm{CH}$ groups: the carbons bonded by a $\pi$-type bond are in a smaller distance between them than those bonded by a $\sigma$-type bond. This process is termed dimerization. Experimental studies showed the existence of two isomers for the polyacetylene: the trans-polyacetylene (tPA) Fig. 1.1(a), more stable from the thermodynamic point of view, and the cis-polyacetylene (cPA) Fig. 1.1(b) [5-7]. For both configurations, experiments showed the existence of unpaired electrons whose mobility was higher in the tPA isomer. In addition, samples of the tPA isomer exhibited metallic levels for the conductivity when they were exposed to high concentrations of dopants [11]. On the other hand, samples of cPA, for similar dopant concentrations, presented quite lower conductivity [11]. Moreover, it was found that the doping process is reversible, i. e., the polyacetylene film regained its original properties by decreasing the impurities concentration [5]. This fact allowed the conductivity degree of the sample to be precisely controlled. $[12,13]$.

(a)<smiles>CC=CC=CC=CC(C)(C)C=CC</smiles><smiles>C/C=C\C=C/C=C\C=C/C=C\C</smiles>

(c)<smiles>CC=Cc1ccc(C)cc1</smiles>

Figure 1.1: Schematic representation of three conjugated polymers: (a) transpolyacetylene, (b) cis-polyacetylene, and (c) poly ( $p$-phenylene vinylene).

Thereafter, many conducting polymers, with more complex structures, were synthesized. However, one fundamental feature was common to all: the alternating 
between single and double bonds, i. e., these complex structures are also conjugated polymers. The poly $(p-$ phenylene vinylene) (PPV), which its structure is represented in Fig. 1.1(c), for example, is a conjugate polymer widely used in the fabrication of OLEDs recently, due to the high photoluminescence yield it possesses.

\subsection{Applications}

Organic semiconductors have attracted considerable interest as potential candidates for the development of optoelectronic devices due mainly to cheap and easy processability. This makes them more attractive materials for the electronics industry to provide a new display technology and benign environmental sources of power than their inorganic counterparts [21]. Very recently, they have been successfully implemented as the active component in applications such as OLEDs [22, 23] and OSCs [24,25]. In these devices, the charge carrier recombination and the generation of excited sates are the key steps behind the mechanism of electroluminescence in OLEDs and the power generation efficiency in OSCs. Following, we detail the working principles of these two major optoelectronic devices.

\subsubsection{Organic Light Emitting Diodes (OLEDs)}

Since the discovery of the generation of light upon an electrical excitation (electroluminescence process) in acridine films in early 60's [26-29], the research field of organic-based materials to the development of a new class of optoelectronic devices has experienced a significant growth. Also, it was found that organic molecular crystals, such as anthracene, requires electric field strengths significantly above of 1.0 $\mathrm{mV} / \AA$ to activate luminescence [30,31]. In the late 80's, the group of Prof. Richard Friend obtained the same phenomenon with a conjugated polymer (PPV), thus promoting a huge breakthrough in this research field [12]. From that moment on, the possibility of these applications to arrive in the market through large companies became reality and the development in the science and technology of OLEDs kept growing tremendously.

In contrast to the conventional inorganic systems, the fact that organic semiconductors are quasi-one-dimensional materials leads to the novel property of its lattice structure being easily distorted to form self-trapped elementary excitations [32]. This can be accomplished either by charge injections or by a photoexcitation mechanism, and results on the induction of self-localized electronic states, such as excitons [32]. In organic semiconductors, an exciton is a bound electron-hole pair state formed due 
to the strong electron-lattice interactions. It is known that the efficiency of OLEDs is related to the exciton dissociation mechanism [12,13]. An OLED normally consists on a luminescent and a conducting conjugated polymer layer, introduced between two metal electrodes. Electrons and holes are injected from the electrodes into the polymer layer; as a result, this process induce self-localized electronic states, which leads to the formation of excitons [12]. In these materials, excitons are generally considered to be more strongly localized than excitons in three-dimensional semiconductors, especially because in the former, the exciton is substantially confined to a single polymer chain [13].

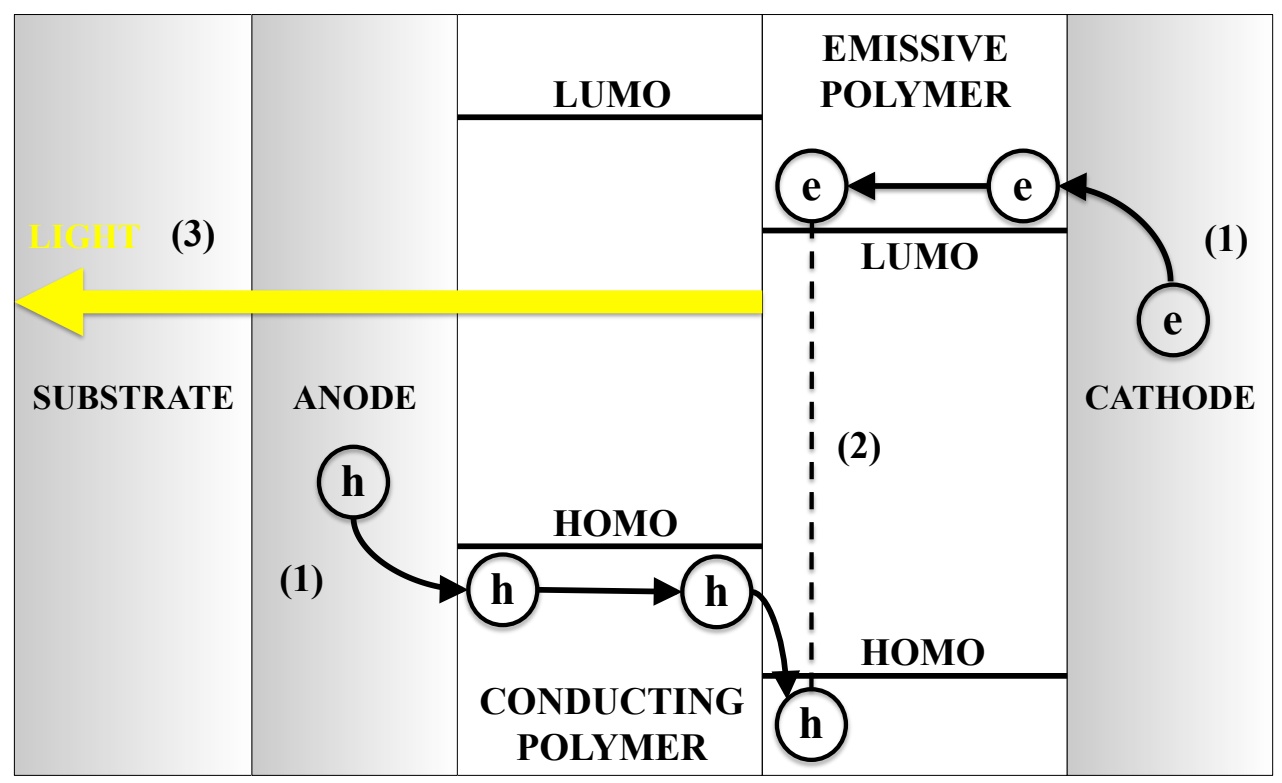

Figure 1.2: A schematic representation of the working principle of an OLED.

A schematic energy-level diagram for an OLED together with its working principle, that represents the exciton dissociation mechanism in this material, is shown in Figure 1.2. This phenomenon is accomplished as follows: first, electrons and holes are injected from negative (anode) and positive (cathode) electrodes respectively (step 1, in the figure). This process is facilitated by choosing a material with low work function for the cathode, similar in energy to the lowest unoccupied molecular orbital (LUMO) of the emissive layer, and by choosing a material with high work function material, similar in energy to the highest occupied molecular orbital (HOMO) of the conductive layer [33]. Following, electrons and holes capture one another within the emissive polymer film, and form neutral bound excited state (step 2). Since the electron mobility is usually slightly lower than the hole mobility in organic semiconductors, the 
recombination occurs closer to the cathode in the emissive layer [33]. Finally, a radiative decay of the excited electron-hole state produced by the recombination process take place (step 3).

The spin wavefunction of the exciton, formed from the two spin- $1 / 2$ electronic charges, can be either singlet $(S=0)$ or triplet $(S=1)$. The radiative emission (fluorescence) comes from the singlet only, and when the exchange energy is large, cross-over from triplet to singlet is unlikely to happen, so that triplet excitons do not produce light emission other than by indirect processes such as phosphorescence or by triplet-triplet annihilation [33]. In OLEDs, the emissive polymer layer generally is formed by a PPV film, which has high photoluminescence yields [13]. The hole transport layer (conducting polymer) widely used in this devices is the Polyaniline (PANI:PPS) [13]. Indium-tin Oxide (ITO) is used as the hole-injecting electrode and a magnesium-silver alloy plays the role of the electron-injecting electrode [13].

\subsubsection{Organic Solar Cells (OSCs)}

The photovoltaic effect also involves the generation of electron and hole pairs and their subsequent collection at opposite electrodes. A key aspect in the physics of organic compared to inorganic semiconductors is the difference on the nature of the optically excited states. Whereas in inorganic materials, the production of free charge is carried out directly, the absorption of a photon in organic materials leads to the formation of an exciton due to the strong electron-lattice interactions [34]. The organic exciton binding energy is naturally large, on the order of (or even larger than) $500 \mathrm{meV}$. This binding energy represents twenty or more times the thermal energy at room temperature, $k_{B} T(300 K)=26 \mathrm{meV}$ to be compared with a few $\mathrm{meV}$ in the case of inorganic semiconductors [35].

A schematic energy-level diagram for a OSC under illumination, that represents the exciton dissociation mechanism, is shown in Figure 1.3. As a first step, the photons are absorbed with an average photon energy larger than the optical band gap on both sides of the heterojunction (step1). Following, the thermalization and the formation of excitons takes place (step 2). The next stage is the excitons diffusion through the heterojunction (3). Finally (4) these structures are dissociated, therefore transferring an electron (hole) into the acceptor (donor) layer. The energy difference between the HOMO and LUMO levels of the donor and acceptor layers denotes, respectively, the energy offset between the ionization potential values (HOMO energies), of the donor molecular layer, and electron affinities (LUMO energies) of the acceptor molecular layer [34]. Figure 1.3 represents a OSC whose acceptor layer is formed by materials 
which have high electron affinity and favors the rapid exciton dissociation, such as $\mathrm{C}_{60}$ crystals, thus resulting in high-power conversion efficiencies [35]. The donor layer widely used in these devices is a Pentacene crystal [35]. The hole and electroninjecting electrodes are normally the same as used in the fabrication of OLED devices [34]. The working mechanism of OSCs shows that the optical absorption in materials based on organic semiconductors does not lead directly to free electron and hole carriers that could readily generate electrical current [34]. Consequently, to generate current in OSCs, the excitons must first dissociate into free charges.

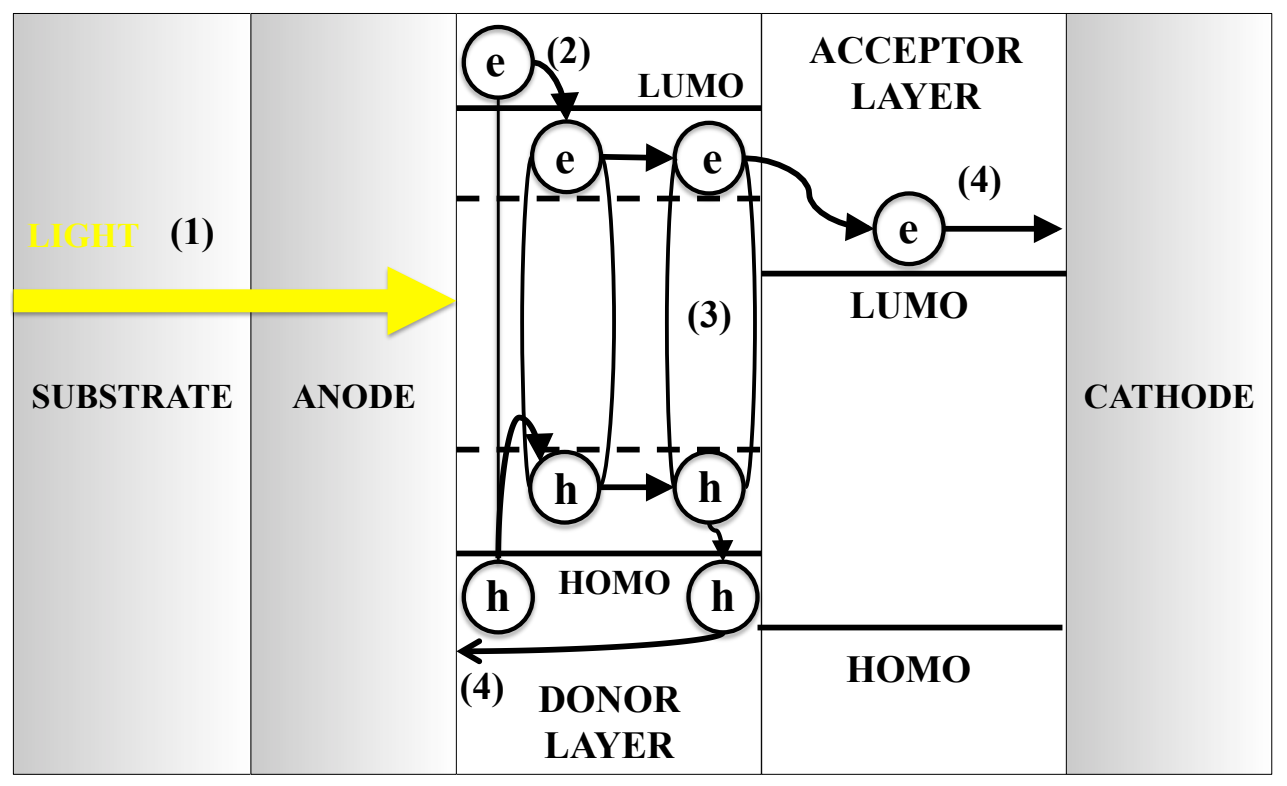

Figure 1.3: A schematic representation of the working principle of an OSC.

\subsection{The State of the Art}

As mentioned above, an OLED normally consists of a luminescent conjugate polymer layer, introduced between two metal electrodes. Electrons and holes are injected from the electrodes into the polymer layer and, as a result, this process also induces selflocalized electron states called polarons [32]. A polaron has spin $\pm 1 / 2$ and a charge \pm e. It is known that the injected electrons and holes forms electron-polarons and hole-polarons due to the strong electron-lattice interactions in these materials [13]. Bipolarons, that are spinless charge carriers and possess charge $\pm 2 \mathrm{e}$, can be created in OLEDs when the charge injection results in a large concentration of polarons [13]. For example, two acoustic polarons with the same charge and antiparallel spins can 
combine with each other to for an acoustic bipolaron [32]. More details about these structures will be provided in Chapter 3 .

When an electron-polaron (-bipolaron) meets a hole-polaron (-bipolaron), they may collide and recombine to form a mixed state composed by polarons (bipolarons) and excitons, in which the electron and the hole are bonded in a self-trapped lattice deformation known as exciton (biexciton), in analogy to conventional excitons in inorganic semiconductors [33]. The photon emission results from the radiative decay of the excitations. Thus, the yield of these excitations determines the electroluminescence efficiency in conjugated polymers [13]. It is known that the recombination process between oppositely charged carriers [36-44] and between charge carries and excitons $[45,46]$ plays an important role in the electroluminescence of OLEDs. Also, it has been generally accepted that the electron-electron interactions $[47,48]$, the temperature effects $[49,50]$, and the presence of impurities [51-53] in a conjugated polymer lattice critically affects the polaron and bipolaron dynamics. In this way, the recombination process of oppositely charged carriers, mainly in the presence of impurities and temperature effects, and the consequent yield of neutral excitations are believed to be of fundamental importance for OLEDs. Nevertheless, studies that take into account this physical picture remain not well described in the literature.

Indeed, one factor of major importance is the role played by Coulomb interactions in the recombination process between the quasi-particles. Lei et al. studied the dependence of exciton formation rate on the spin orientation of polarons by simulating the collision process of two oppositely charged quasiparticles, using a modified version of $\mathrm{Su}$-Schrieffer-Heeger (SSH) model [41]. The role played by the spin configuration in the collision dynamics of these charge carriers is a question of fundamental importance for electroluminescence processes in OLEDs. Their results show that the yield of singlet excitons from parallel spin or antiparallel spin polarons configuration is not influenced by the electron-electron (e-e) interactions. On the other hand, in the manufacture of spin OLEDs to improve the luminescent efficiency, the e-e interactions should be critical factors, whose role must be clarified. Particularly, understanding how internal effects such as e-e interactions and impurity effects can favor or unfavor the polaron-exciton formation via scattering of oppositely charged polarons is an issue that is believed to be crucial for the design of more efficient devices with respect to the electroluminescence. Thus, this point requires a better phenomenological description.

Some relevant theoretical studies carried out by An and co-workers have shown that external electric field has a significant influence on polaron-exciton formation via polaron-pair scattering in conjugated polymers [36]. The goal was to identify the 
generation mechanism of the self-trapped polaron-exciton. Their results show that, for the scattering processes of two polarons initially presented on a same polymer chain, three regimes of the applied electric field strength were identified: (1) at field strength smaller than $0.2 \mathrm{mV} / \AA$, the polaron-pair scatter into an exciton state after $1300 \mathrm{fs}$; (2) for an electric field strength between $0.2 \mathrm{mV} / \AA$ and $1.2 \mathrm{mV} / \AA$, the polaron-pair scatters into a pair of independent particles and each of them is a mix of polarons and excitons (in this case, the yield of the neutral exciton depends sensitively on the electric field strength); and (3) at electric field strength greater than $1.2 \mathrm{mV} / \AA$, the two polarons break into irregular lattice vibrations after their collision. When two polymer chains are taken into account, the two polarons will combine together to form a self-trapped exciton in one of the chains when they lie initially on different polymer chains. These results indicate that the interchain interaction favors the formation of polaron-excitons.

Still considering the recombination dynamics between two oppositely charged polarons, Li and colleagues expanded the process as in studies carried out by An and collaborators [36], by adding Hubbard type e-e interactions to the SSH Hamiltonian [44]. The simulations have pointed out that two polarons can recombine directly and efficiently in a full exciton, a process that is strongly dependent on the field strength. Sun and Stafström have used the SSH model to investigate the formation of excited states through the recombination process between two oppositely charged polarons in a system composed of two coupled conjugated polymer chains [37]. In this case it is reported that, depending on the interchain distance and the electric field strength, the two polarons can either recombine into an exciton, become bound together forming a polaron pair or pass each other. Furthermore, it was found that the singlet states are always more easily formed than the triplet ones. These facts indicate that in OLEDs, the electroluminescence quantum efficiency can exceed the statistical limitation value of $25 \%$.

Recently, it was experimentally reported that the capture of a positive bipolaron by a deeply trapped negative polaron is one of the mechanism of trion formation in a PPV derivative [54]. Sun and collaborators investigated theoretically the recombination process between an oppositely charged polaron-bipolaron pair in conjugated polymers using a modified version of the SSH model [39]. They have pointed out that below a critical electric field, the polaron and bipolaron can scatter into an excited polaron with high yield. Above the critical electric field, the polaron and bipolaron will pass through each other and continue moving as isolated quasi-particles. Through the use of a similar approach, the results obtained by Onodera showed that polarons and 
bipolarons can freely pass through each other without attractive or repulsive interactions [55]. Theoretical studies carried out by Silva have shown that the presence of a bipolaron limits the polaron movement in such a way that the polaron cannot pass through the bipolaron keeping its integrity after the nonreactive collisional process, thus resulting in a physical picture where the polaron mobility is compromised [56].

Studies carried out by Di and co-workers have shown that external electric field strength has a significant influence on the results of bipolaron-bipolaron and bipolaronpolaron interactions via scattering processes in conjugated polymers $[42,43]$. By using a modified version of the SSH model to include the Brazoviskii-Kirova symmetry breaking term, e-e interactions, and an external electric field they have investigated the scattering and combination of oppositely charged bipolarons in a conjugated polymer monolayer and in OLED heterojunction systems. Their results show that two bipolarons can scatter into singlet biexciton state in monolayer and/or multiple-layer polymeric materials. The yield of biexcitons in the collision of bipolarons can reach values as high as about $75 \%$. The biexciton state can decay to an exciton state, which can subsequently decay to the ground state, allowing the overall electroluminescent quantum efficiency in OLEDs from all sources to reach values as high as about $75 \%$. These results contributed to the understanding of the experimental fact that the internal quantum efficiency can reach $60 \%$. Recently, Sun and Stafström have simulated the same process depicting the effects of the e-e interactions for a system composed of two coupled conjugated polymer chains [38]. Their findings indicate that there are four channels for the bipolaron recombination: (1) forming a biexciton, (2) forming an excited negative polaron and a free hole, (3) forming an excited positive polaron and a free electron, and (4) forming an exciton, a free electron and a free hole.

The dynamics of generation and recombination of charge carriers in polymer films of poly-para-phenylene (m-LPPP) by means of femtosecond electromodulated transient absorption spectroscopy were examined in Ref. [57]. Using this method, two modes of polaron formation from relaxed exited states (excitons) with different behaviors were identified: (i) impurity induced in the absence of an external electric field and (ii) electric field induced in a pristine film. It was also observed that only a small part of excitons generates charge carriers at zero applied field. The main portion of polarons recombines faster, but a fraction survives up to microseconds and form fluorescent excited states. This fact shows the difficulty in keeping free charge carriers stable enough to generate useful electrical current. Moses and colleagues analyzed the charge carrier generation and relaxation dynamics using transient excited state absorption (photo-induced absorption) measurements in a prototypical 
luminescent polymer (PPV), probed with 100 fs temporal resolution in the $6-10 \mathrm{~mm}$ spectral region. They spanned the infrared active vibrational modes to propose the direct polaron creation, and also to indicate that the charge carrier dissociation rate depends on the strength of the interchain interaction. This result suggests that direct polaron creation can be an alternative route to the generation of stable free charge carriers.

Theoretical studies on the effects of interchain interaction on the recombination processes of charge carrier and on the generation dynamics in polymer chains have been extensively carried out recently $[58,59]$. In these studies, a nonadiabatic evolution method was used to investigate the relaxation dynamics of the charge carriers in the presence of an external electric field. The inclusion of electron repulsion terms has provided very interesting results concerning excitons with a SSH type model for parallel chains. Considering a pair of interacting chains, the results showed that there are not only intrachain excitons, but also interchain excitons. By an intrachain exciton we mean a structure that contains, in a single chain, an electron coupled to a hole, while an interchain exciton consists of a bound state of a positive polaron on one chain and a negative polaron on the neighboring chain. Also, the results suggest that the creation efficiency of interchain excitons increases by increasing the interchain coupling, while that of intrachain excitons decreases.

It is important to emphasize here that all above-mentioned studies have been focused on specific cases with idealized conditions. A theory that widely holds true for real materials needs further verifying by addressing some realistic effects, for example, the order degree of molecules, temperature and impurity effects. From these works, we can see that all the results for the polaron-pair interaction are not fully described, so that further investigations that take into account some of these effects are needed. Particularly, a feature specially not appropriately described in the literature is the temperature influence on the recombination process of charge carriers. Although thermal effects are known to be important to the charge carriers creation mechanism, recent theoretical and experimental studies suggest that the photogeneration creation time is temperature-independent. Besides, experimental evidence suggests that the dynamical decay of transient photoconductivity is temperature-independent [60-62]. These results suggest that the contribution of the localized charge carriers (polarons) to the photocurrent is predominant at higher time scales. It is worth to mentioning, also, that a consistent study about the products of the recombination processes between quasi-particles, particularly concerning features that might increase the yield 
on the formation of excited states, had not been performed, so that further investigations are needed.

A final remark concerning the system investigated in this work must be performed. Our work report mainly results concerning polyacetylene species in the cis configuration. One, however, should have noticed the great amount of different compounds that present high expectation of applicability in the organic electronic field. As we will soon discuss, the model applied to treat conjugated polymers is a parametrical semi-empirical tight-binding with lattice relaxation. Therefore, although we are, in principle, dealing with cis-polyacetylene, a simple change of parameters obtained experimentally would be enough to address any kind of $\pi$-conjugated polymer with the cis symmetry (which, incidentally, is the dominating symmetry as far as applicability is concerned). By not doing so, however, we can expect our results to have an important generality feature, typical of those models applies to standard systems. In order words, we developed a general model to be used for any particular kind of system, provided the correct parameters are included. Experience shows that the qualitative behavior of the electronic transport differs very little between different conjugated polymers, because the alternating pattern is pretty much the same and the coupling between $\pi$ electrons and lattice is responsible for the transport. Therefore, our results are expected to be qualitatively valid for conjugated polymers in general.

\subsection{Thesis Outline}

The present and the following chapters are intended to serve as a further introduction to the papers included in this thesis. A short historical review regarding the discovery of conducting polymers were presented in the present chapter. An introduction about $\pi$-conjugated molecular systems, particularly the polyacetylene, was provided as well as a list of some important applications of charge recombination in organic semiconductors. As a motivation, we have just discussed the state of the art referring to this issue from the framework of a tight-binding approach. In Chapter 2, the theoretical background referring to the approaches used here are provided. Finally, in Chapter 3 the Su-Schrieffer-Heeger (SSH) model and its extensions to include an external electric field, coulombic interactions, impurities, and temperature effects are extensively described. The scientific papers constitute the second part of this thesis and are presented in the following together with a short summary of their goals. In blue, are the links to the websites of the papers for the online version of the thesis: 


\section{Paper I}

The purpose of this paper was to propose a new method, using the genetic algorithm optimization technique (GAOT), designed to obtain the lattice geometry parameters and the electronic structure of $\pi$-conjugated polymer systems. Particularly, we have obtained the ground state structure of a completely dimerized trans-polyacetylene. Moreover, we have used this approach to determine the polaron and soliton solutions. These solutions were then compared to the traditional self-consistent-field (SCF) solutions. We have concluded that the optimization technique proposed here generates solutions as those obtained using traditional (SCF) techniques, with the important advantage of reducing significantly the number of iterations needed to reach convergence.

\section{Paper II}

The stability of charge carriers in conjugated polymers was investigated in terms of a nonadiabatic evolution method by using an extended version of the SSH model that includes the effects of an external electric field and temperature. On the basis of this physical picture, different patterns of applied electric field and temperature dependence of polaron and bipolaron kinematics as well as the transitions between different regimes are found. Phase transitions from subsonic to supersonic velocities are also discussed in terms of the system conditions. We were able to describe at which thermal regime each quasi-particle loses its stability and also to determine under which circumstances do the electric field and temperature rise or dampen its motion. These results are particularly important in order to enlighten the understanding of the temperature-included recombination mechanism between oppositely charged carriers.

\section{Paper III}

The third paper turned out to be the first of a series of ten papers, in which the recombination processes between charge carriers and excited states were taken into account. For this paper, the exciton dissociation and charge recombination processes in organic semiconductors, considering the influence of a thermal bath, are described. Here, we analyzed the mechanisms of polaron-excitons dissociation into free charge carriers and the consequent recombination of those carriers under thermal effects on 
two parallel $\pi$-conjugated polymers chains electronically coupled. Our findings suggest that exciton dissociation in a single molecule gives rise to localized, polaron-like charge carrier. Besides, we concluded that in the case of interchain processes, the bimolecular polaron recombination does not lead to an usual exciton state. Rather, this type of recombination leads to an oscillating dipole between the two chains. Finally, our results show that temperature effects are essential to the relaxation process leading to polaron formation in a single chain, as in the absence of temperature, this process was not observed. In the case of two chains, we conclude that temperature effects also favors the bimolecular recombination process, as observed experimentally.

\section{Paper IV}

This paper deals with the temperature influence on the exciton dissociation dynamics in conjugated polymer systems. Using a modified version of the SSH model, the dissociation is studied under the influence of impurity effects with a nonadiabatic evolution method. Our findings have shown that temperature effects reduces the critical electric field necessary for the exciton dissociation.

\section{Paper V}

In the fifth paper, we focus on the collision between two oppositely charged polarons under the influence of impurity effects combining the SSH model and the extended Hubbard model. Our findings show that e-e interactions have direct influence on the charge distribution coupled to the polaron-exciton lattice defect. Additionally, the presence of an impurity in the collisional process reduces the critical electric field for the polaron-exciton formation. In the small electric field regime, the impurity effects open three channels and are of fundamental importance to favor the polaron-exciton creation.

\section{Paper VI}

For this paper, the scattering of two oppositely charged bipolarons and of a bipolaronpolaron pair was investigated under the influence of impurity effects using a modified version of the SSH model that includes e-e interactions. The novel results for bipolarons show that these oppositely charged quasi-particles can scatter into a mixed state composed of bipolarons and excitons. The excitation yield depends sensitively 
on the strength of the applied electric field. In the presence of an impurity, the critical electric field regime for formation of a state composed by bipolarons and excitons is increased. Additionally, we were able to obtain critical values of electric fields that played the role of drastically modifying the system dynamics.

\section{Paper VII}

Using a modified version of the SSH model combined with the extended Hubbard model (EHB), the recombination between a singlet exciton pair was investigated under the influence of an external electric field, e-e interactions, and temperature effects. The excitons were positioned very close to each other in a way to mimic a high-density region in monomolecular conjugated polymer systems. Results show that there are mainly three possible channels resulting from singlet-singlet exciton recombination: (1) forming an excited negative polaron and an excited positive bipolaron, (2) forming two free and excited oppositely charged polarons, and (3) forming a biexciton.

\section{Paper VIII}

The scattering process between an electron-polaron and a hole-bipolaron has been simulated using a version of the SSH model modified to include an external electric field, Coulomb interactions, and temperature effects in the scope of nonadiabatic molecular dynamics. The simulations reveal remarkable details concerning the polaron-bipolaron recombination reaction. It is found that there exists a critical temperature regime below which a hole-bipolaron and a mixed state composed by an electron-polaron and an exciton are formed and a hole-bipolaron and a free electron are the resulting products of the collisional process, if the temperature is higher than the critical value. In addition, it is obtained that both channels depend sensitively on the strength of the applied electric field.

\section{Paper IX}

The intrachain recombination dynamics between oppositely charged polarons is theoretically investigated through the use of a version of the SSH model modified to include an external electric field, an extended Hubbard model, Coulomb interactions, and temperature effects in the framework of a nonadiabatic evolution method. Our results indicate notable characteristics concerning the polaron recombination: (1) it 
is found that there exists a critical temperature regime, below which an exciton is formed directly and (2) a pristine lattice is the resulting product of the recombination process, if the temperature is higher than the critical value. Additionally, it is found that the critical electric field regime plays the role of drastically modifying the system dynamics.

\section{Paper X}

The temperature influence over the intrachain recombination dynamics between oppositely charged bipolarons has been theoretically investigated using a version of the SSH model. The results show that, below a critical temperature regime, these charge carriers can recombine into a biexciton. This excited state is a light emissive specie that can emit one photon and turn into an exciton state. This specie can thereafter decay to the ground state by other photon emission.

\section{Paper XI}

The temperature influence on the scattering process between an exciton and a positively charged carrier (polaron or bipolaron) is theoretically investigated using a version of the SSH model modified to include temperature, Coulomb interactions, and an external electric field. In general, it is observed that the products of the reactive scattering are spin independent when thermal effects are taken into account. For the interaction between a polaron and an exciton, the polaron can be annihilated, when subjected to temperatures higher than a critical value, or pass through the exciton maintaining both their consistencies, if a lower temperature regime is considered. Regarding to the recombination between a bipolaron and an exciton, it is observed that the bipolaron can be annihilated whereas the exciton dissociates into two trions or into one polaron and one trion. In all cases, the recombination mechanisms depend upon of a suitable balance between temperature and electric field.

\section{Paper XII}

The impact of the charge carrier density on the recombination dynamics between oppositely charged polarons is numerically investigated using a modified version of the SSH model that includes an external electric field, and e-e interactions. Our findings 
show that the interplay between polaron density and electric field can avoid the formation of excited states (polaron-exciton and neutral excitation) leading the system to a pristine lattice. On the other hand, depending on a suitable balance between these two properties, the recombination mechanism can form stable polaron-excitons or neutral excitations. Interestingly, the polaron density plays the role to define the products formed from the recombination process, in which these two kind of excited states hardly coexist.

\section{Paper XIII}

In the last paper, the field induced dynamics of quasi-polarons in armchair graphene nanoribbons (GNRs) is theoretically investigated in the framework of a tight-binding approach with lattice relaxation. Our findings show that the semiconductor band gap behavior, which is fundamental to the quasi-polaron transport to take place, depends upon of a suitable balance between the GNR width and the electron-phonon (e-ph) coupling strength. Moreover, it is found that the space parameter for which the quasi-polaron is dynamically stable is quite limited to variations in the e-ph coupling value and strongly dependent on the GNR width. Interestingly, the interplay between the external electric field and the e-ph coupling plays the role to define a phase transition from subsonic to supersonic velocities for the quasi-polaron structure. 


\section{CHAPTER}

\section{Theoretical Background}

The present chapter is intended to serve as an introduction to the theoretical methods referring to the approach used to perform the simulations. The chapter begins by considering the many-body problem. Following, it is presented the form to treat the states according the antisymmetry of the wavefunction. Moreover, a brief overview about the second quantization formalism as well as the tight-binding model is provided in order to present the most convenient treatment for the system. Finally, for the sake of clarity, the Langevin formalism is presented to show how the temperature effects are included in our model.

\subsection{The Many-Body Problem}

Conducting polymers are molecules formed from smaller structural units (monomers), such as a CH group [63]. These monomers, in their turn, are composed by electrons and cores, so characterizing a many-body system [63,64]. In this way, the quantum treatment of this system is performed using the time-dependent Schrödinger equation,

$$
i \hbar \frac{\partial}{\partial t}|\Psi\rangle=\mathcal{H}|\Psi\rangle
$$

The system may be represented as shown in Figure 2.1, where the distance between two electrons, $i$ and $j$, is given by $r_{i, j}=\left|r_{i}-r_{j}\right|$, whereas the distance between two cores, $A$ and $B$, can be defined as $R_{A B}=\left|R_{A}-R_{B}\right|$ [65]. In its turn, the distance between an electron and a core is represented, for example, as $r_{i A}=\left|r_{i}-R_{A}\right|$ [65]. Thereby, the electronic and nuclear degrees of freedom are described by a many-body Hamiltonian $(\mathcal{H})$,

$$
\mathcal{H}=\mathcal{H}_{c-c}(\{\mathbf{R}\})+\mathcal{H}_{e-e}(\{\mathbf{R}\})+\mathcal{H}_{e-c}(\{\mathbf{r}, \mathbf{R}\}) .
$$

$\mathcal{H}_{c-c}$ denotes the interaction energy between the cores, i. e., the core kinetic energy and its potential energy from the Coulomb interactions. The term $\mathcal{H}_{e-e}$ establishes 


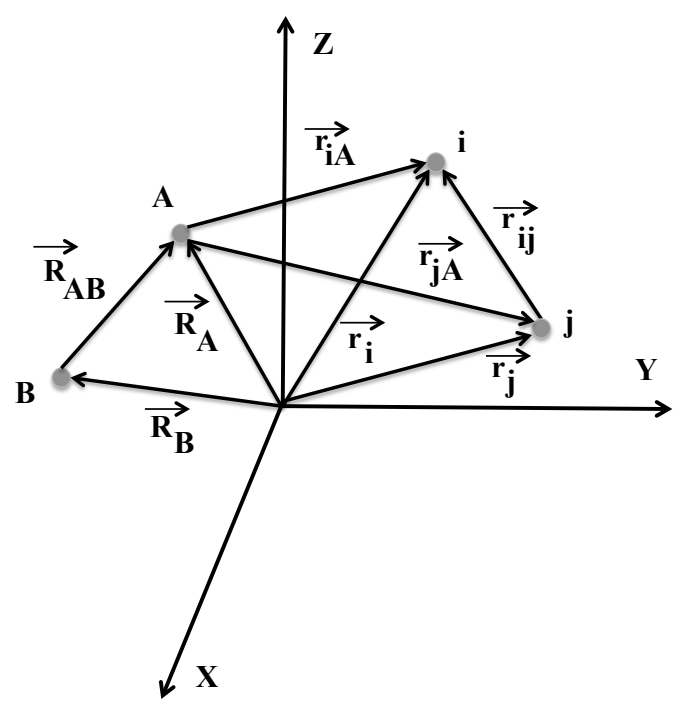

Figure 2.1: Molecular coordinate system for the many-body problem.

the energy from the electron-electron interactions, including also its kinetic energy. Finally, the term $\mathcal{H}_{e-c}$ describes the potential energy due to the Coulomb interactions between electrons and cores. Thus, $\mathcal{H}$ can be placed, using atomic units, in the following form

$$
\mathcal{H}=-\sum_{i=1}^{N} \frac{1}{2} \nabla_{i}^{2}-\sum_{A=1}^{M} \frac{1}{2 M_{A}} \nabla_{A}^{2}-\sum_{i=1}^{N} \sum_{A=1}^{M} \frac{Z_{A}}{r_{i A}}+\sum_{i=1}^{N} \sum_{j>i}^{N} \frac{1}{r_{i j}}+\sum_{A=1}^{M} \sum_{B>A}^{M} \frac{Z_{A} Z_{B}}{R_{A B}}
$$

where $M_{A}$ is the mass for the core $A$ and $Z_{A}$ is its atomic number [65].

The first of these operators represents a sum of one-particle operators, which has the form

$$
O_{1}=\sum_{i=1}^{N} h(i)
$$

where $h(i)$ is any operator involving only variables of the $i$-th particle. In this case, this operator can be placed in the form

$$
\mathcal{H}_{1}=-\sum_{i=1}^{N} \frac{1}{2} \nabla_{i}^{2}-\sum_{A=1}^{M} \frac{1}{2 M_{A}} \nabla_{A}^{2}
$$

In other words, this kind of operator contains dynamical variables that depend only on the position or momentum of the particle [65]. The first term in this operator describes the kinetic energy of the electrons whereas the the second one refers to the kinetic energy for the cores. 
The second kind of operator considered in the scope of the many-body theory can be obtained by summing of two-particles operators as

$$
O_{2}=\sum_{i=1}^{N} \sum_{j>i} v(i, j)
$$

which $v(i, j)$ represents any operator that depends on the position or momentum of the $i$-th and $j$-th particles [65]. Naturally, this class of operators can be represented using the equation

$$
\mathcal{H}_{2}=\sum_{i=1}^{N} \sum_{A=1}^{M} \frac{Z_{A}}{r_{i A}}+\sum_{i=1}^{N} \sum_{j>i}^{N} \frac{1}{r_{i j}}+\sum_{A=1}^{M} \sum_{B>A}^{M} \frac{Z_{A} Z_{B}}{R_{A B}},
$$

which is composed by terms that establishes the Coulomb interactions. The first part in Eq. 2.7 refers to the electron-core attraction whereas the two last terms describe the electron-electron and core-core repulsions, respectively.

\subsection{Antisymmetric Wavefunctions and the Slater de- terminant}

The principle of indistinguishability of a system of fermions requires the wavefunction, that describes the many-electrons system, to be antisymmetric regarding an inversion in the $\mathrm{x}$-coordinates (position and spin) of two electrons [65], in order that

$$
\Psi\left(x_{1}, \ldots x_{i}, \ldots x_{j} \ldots x_{N}\right)=-\Psi\left(x_{1}, \ldots x_{j}, \ldots x_{i}, \ldots x_{N}\right) .
$$

As the antisymmetry is an intrinsic feature of the determinants, a convenient manner of express the electronic wavefunction, for a many-body system, is to expand it in terms of a complete set of functions, named Slater Determinants [65]. These determinants may be defined as mathematical tools used in quantum mechanics to generate antisymmetric wavefunctions that describe the collective states of several fermions [66].

For a complete description of the electron, it is mandatory to specify its spacial and spin degrees of freedom. In order to do so, two orthonormal functions $\alpha(\omega)$ and $\beta(\omega)$, i. e., spin-up and spin-down functions, respectively are defined. The one-electron wavefunction that simultaneously describes both spacial $(\psi(\mathrm{x}))$ and spin $(\alpha(\omega)$ or $\beta(\omega))$ distributions is known as spin orbital $\chi(\mathrm{x})$, $\mathrm{x}$ standing for a set with both coordinates (spacial and spin). Thus, a spin orbital my be characterized as

$$
\chi(x)=\left\{\begin{array}{l}
\psi(r) \alpha(\omega) \\
\text { or } \\
\psi^{\prime}(r) \beta(\omega) .
\end{array}\right.
$$


The spacial orbital is a function of the position vector $r$. In this way, such coordinate describes the spacial distribution of an electron, with the probability of finding it in a certain volume element $d r$, around $r$, given by $\left|\psi_{i}(r)^{2}\right|$. As the spin and spacial orbitals are orthonormal, the following relation can be written

$$
\begin{aligned}
& \int \psi_{i}^{*}(r) \psi_{j}(r) d r=\delta_{i j} \\
& \int \chi_{i}^{*}(\mathrm{x}) \chi_{j}(\mathrm{x}) d r=\delta_{i j} .
\end{aligned}
$$

Knowing that the spin orbital is a convenient wavefunction to the description of an electron, we can now turn to the case where wavefunctions for a set of electrons are considered. For a N-electrons wavefunction, where the mutual interaction is not considered initially, the Hamiltonian $\left(\mathcal{H}_{1}\right)$ assumes the form of Eq. 2.4. Therefore, the operator $h(i)$ has now a set of eigenfunctions that can be identified as a set of spin orbitals $\left\{\chi_{j}\right\}$,

$$
h(i) \chi_{j}\left(\mathrm{x}_{i}\right)=\epsilon_{j} \chi_{j}\left(\mathrm{x}_{j}\right) .
$$

Due to the fact that $\mathcal{H}_{1}$ is a sum of one-electron Hamiltonians, the $\mathrm{N}$-electrons wavefunction is given by the product of the spatial and orbital wavefunctions for each electron, where

$$
\Psi_{P H}\left(\mathrm{x}_{1}, \ldots \mathrm{x}_{i}, \ldots \mathrm{x}_{j} \ldots \mathrm{x}_{N}\right)=\chi_{i}\left(\mathrm{x}_{1}\right) \chi_{j}\left(\mathrm{x}_{2}\right) \ldots \chi_{k}\left(\mathrm{x}_{N}\right)
$$

The electronic problem can be solved assuming that the electrons do not interact. Another solution for this problem would be to define that $h_{i}$ can be the one-electron Hamiltonian, that includes the electron-electron interactions in average form, once that a constant summed to the Hamiltonian does not alter the solution [65]. The $\Psi_{P H}$ is known as Hartree Product, where $\Psi_{P H}$ is an eigenfunction of $\mathcal{H}_{1}$ [65]. Thus,

$$
\mathcal{H}_{1} \Psi_{P H}=E \Psi_{P H}
$$

The eigenvalue $E$ is the sum of the spin orbital energies for each one of them contained in $\mathcal{H}_{1}$,

$$
E=\epsilon_{i}+\epsilon_{j}+\ldots+\epsilon_{k}
$$

It is noted that the Hartree Products do not satisfies the antisymmetry principle. However, it is possible to obtain antisymmetric wavefunctions according with the following discussion. Initially, it is considered a system containing two electrons which 
occupy the orbitals $\chi_{i}$ and $\chi_{j}$. The wavefunction that describes the configuration with the first electron being in the $\chi_{i}$ orbital and the other one in $\chi_{j}$, can be described as

$$
\Psi_{12}^{P H}\left(\mathrm{x}_{1}, \mathrm{x}_{2}\right)=\chi_{i}\left(\mathrm{x}_{1}\right) \chi_{j}\left(\mathrm{x}_{2}\right)
$$

Analogously, the wavefunction that represents the configuration in which the second electron is in the orbital $\chi_{i}$, whereas the first electron is in $\chi_{j}$, is given by

$$
\Psi_{21}^{P H}\left(\mathrm{x}_{1}, \mathrm{x}_{2}\right)=\chi_{i}\left(\mathrm{x}_{2}\right) \chi_{j}\left(\mathrm{x}_{1}\right)
$$

It is possible to note that in the Hartree Products $\Psi_{12}^{P H}$ and $\Psi_{21}^{P H}$ there is an distinction between the electrons. The main goal of this procedure is to obtain a wavefunction for which the electrons are indistinguishable and, moreover, satisfies the antisymmetry principle. One function that contemplates such requirements can be built through the linear combination of these Hartree Products [65],

$$
\Phi\left(\mathrm{x}_{1}, \mathrm{x}_{2}\right)=\xi\left[\Psi_{12}^{P H}-\Psi_{21}^{P H}\right]
$$

where

$$
\Phi\left(\mathrm{x}_{1}, \mathrm{x}_{2}\right)=\xi\left[\chi_{1}\left(\mathrm{x}_{1}\right) \chi_{2}\left(\mathrm{x}_{2}\right)-\chi_{1}\left(\mathrm{x}_{2}\right) \chi_{2}\left(\mathrm{x}_{1}\right)\right] .
$$

Also, it is known that

$$
\int \Phi^{*}\left(\mathrm{x}_{1}, \mathrm{x}_{2}\right) \Phi\left(\mathrm{x}_{1}, \mathrm{x}_{2}\right) d \mathrm{x}_{1} d \mathrm{x}_{2}=1
$$

Due to the orthonormality conditions showed in Eq. 2.10, besides of

$$
\begin{aligned}
& \langle\alpha(\omega) \mid \alpha(\omega)\rangle=\langle\beta(\omega) \mid \beta(\omega)\rangle=1 \\
& \langle\alpha(\omega) \mid \beta(\omega)\rangle=\langle\beta(\omega) \mid \alpha(\omega)\rangle=0 .
\end{aligned}
$$

Using the Eq. 2.9, it is obtained that

$$
\Phi\left(\mathrm{x}_{1}, \mathrm{x}_{2}\right)=\frac{1}{\sqrt{2}}\left[\chi_{i}\left(\mathrm{x}_{1}\right) \chi_{j}\left(\mathrm{x}_{2}\right)-\chi_{i}\left(\mathrm{x}_{2}\right) \chi_{j}\left(\mathrm{x}_{1}\right)\right] .
$$

Furthermore, one can see that the wavefunction obtained through the linear combination of Hartree Products is antisymmetric and normalized, where

$$
\Phi\left(\mathrm{x}_{1}, \mathrm{x}_{2}\right)=-\Phi\left(\mathrm{x}_{2}, \mathrm{x}_{1}\right)
$$

Another feature that can be noted is that, if $i=j$ in Eq. 2.21, then the wave function vanishes, i. e., two identical fermions cannot occupy the same quantum state simultaneously, thus agreeing with the Pauli Exclusion Principle. 
The wavefunction expressed in Eq. 2.21 can be written in a determinant form, known as Slater Determinant [65]

$$
\Phi\left(\mathrm{x}_{1}, \mathrm{x}_{2}\right)=\frac{1}{\sqrt{2}}\left|\begin{array}{ll}
\chi_{i}\left(\mathrm{x}_{1}\right) & \chi_{j}\left(\mathrm{x}_{1}\right) \\
\chi_{i}\left(\mathrm{x}_{2}\right) & \chi_{j}\left(\mathrm{x}_{2}\right)
\end{array}\right|
$$

Considering a N-electron system, the generalized form for Eq. 2.23 is

$$
\Phi\left(\mathrm{x}_{1}, \mathrm{x}_{2}, \ldots, \mathrm{x}_{N}\right)=\frac{1}{\sqrt{N !}}\left|\begin{array}{cccc}
\chi_{i}\left(\mathrm{x}_{1}\right) & \chi_{j}\left(\mathrm{x}_{1}\right) & \ldots & \chi_{k}\left(\mathrm{x}_{1}\right) \\
\chi_{i}\left(\mathrm{x}_{2}\right) & \chi_{j}\left(\mathrm{x}_{2}\right) & \cdots & \chi_{k}\left(\mathrm{x}_{2}\right) \\
\vdots & \vdots & & \vdots \\
\chi_{i}\left(\mathrm{x}_{N}\right) & \chi_{j}\left(\mathrm{x}_{N}\right) & \cdots & \chi_{k}\left(\mathrm{x}_{N}\right)
\end{array}\right|
$$

in which $1 / \sqrt{N \text { ! }}$ is a normalization factor. Normally, the Eq. 2.24 can be placed in a compact form in order to highlight the principal diagonal of the determinant [65],

$$
\Phi\left(\mathrm{x}_{1}, \mathrm{x}_{2}, \ldots, \mathrm{x}_{N}\right)=\left|\chi_{i}\left(\mathrm{x}_{1}\right) \chi_{j}\left(\mathrm{x}_{2}\right) \ldots \chi_{k}\left(\mathrm{x}_{N}\right)\right\rangle .
$$

The motivation to use this compact version (which allows to treat more efficiently the many-body problem) will become clear in the next section, where the Second Quantization formalism is introduced.

\subsection{Second Quantization Formalism}

In the second quantization formalism, the antisymmetry property of the wavefunction is related to algebraic features of certain operators, thus being unnecessary to use the explicit form of the determinants [65]. Such formalism is frequently applied to fermionic systems and constitutes a convenient way to treat many-body systems.

Initially, within this approach it is defined a reference state in the Hilbert space, termed "vacuum state" $|\quad\rangle$. This state represents a system without electrons. Therefore, the annihilation operator $a_{i}$ can be defined with respect to its acting on the vacuum state,

$$
a_{i}|\quad\rangle=0
$$

The creation operator, in its turn, which is the adjoint operator of $a_{i}$, is defined by its action on an arbitrary Slater Determinant as

$$
a_{i}^{\dagger}\left|\chi_{j} \chi_{k} \ldots \chi_{l}\right\rangle=\left|\chi_{i} \chi_{j} \chi_{k} \ldots \chi_{l}\right\rangle
$$

Thus, $a_{i}^{\dagger}$ creates an electron in the orbital $\chi_{i}$. It is important to note that the order which the creation and annihilation operators are applied is fundamental, because

$$
a_{i}^{\dagger} a_{j}^{\dagger}\left|\chi_{k} \ldots \chi_{l}\right\rangle=a_{i}^{\dagger}\left|\chi_{j} \chi_{k} \ldots \chi_{l}\right\rangle=\left|\chi_{i} \chi_{j} \chi_{k} \ldots \chi_{l}\right\rangle
$$


and differently,

$$
a_{j}^{\dagger} a_{i}^{\dagger}\left|\chi_{k} \ldots \chi_{l}\right\rangle=a_{j}^{\dagger}\left|\chi_{i} \chi_{k} \ldots \chi_{l}\right\rangle=-\left|\chi_{i} \chi_{j} \chi_{k} \ldots \chi_{l}\right\rangle
$$

For which was employed the antisymmetry principle of the Slater determinant itself. Considering now a sum of the Eqs. 2.28 and 2.29, it is obtained that

$$
\left(a_{j}^{\dagger} a_{i}^{\dagger}+a_{i}^{\dagger} a_{j}^{\dagger}\right)\left|\chi_{k} \ldots \chi_{l}\right\rangle=0
$$

By definition, the Slater Determinant is arbitrary. Therefore,

$$
\left\{a_{j}^{\dagger}, a_{i}^{\dagger}\right\}=a_{j}^{\dagger} a_{i}^{\dagger}+a_{i}^{\dagger} a_{j}^{\dagger}=0
$$

i. e., the anticommutator of any two creation operators is null. Thus,

$$
a_{j}^{\dagger} a_{i}^{\dagger}=-a_{i}^{\dagger} a_{j}^{\dagger}
$$

where to exchange the application order of the operators, it is only necessary to change the signal of the operator $a_{i}^{\dagger} a_{j}^{\dagger}$. Particularly, if the indexes are equal, then

$$
a_{i}^{\dagger} a_{i}^{\dagger}=-a_{i}^{\dagger} a_{i}^{\dagger}=0
$$

This shows that it is not possible to create two electrons in the same orbital. Eq. 2.33 is the form of the Pauli Exclusion Principle in the second quantization formalism [65].

Consider now any state $|Z\rangle$, so that

$$
|Z\rangle=\left|\chi_{i} \chi_{j}\right\rangle=a_{i}^{\dagger}\left|\chi_{j}\right\rangle
$$

The adjoint of this state must be

$$
(|Z\rangle)^{\dagger}=\left(a_{i}^{\dagger}\left|\chi_{j}\right\rangle\right)^{\dagger}=\left\langle\chi_{j}\right|\left(a_{i}^{\dagger}\right)^{\dagger} \equiv\left\langle\chi_{j}\right| a_{i}=\langle Z|
$$

where $|Z\rangle$ is a normalized state

$$
\langle Z \mid Z\rangle=\left\langle\chi_{j}\left|a_{i}\right| \chi_{i} \chi_{j}\right\rangle=1
$$

As $\left\langle\chi_{j} \mid \chi_{j}\right\rangle=1$, in order to keep the formulation coherent, it is necessary to define here

$$
a_{i}\left|\chi_{i} \chi_{j}\right\rangle=\left|\chi_{j}\right\rangle
$$

Thus, we have just the annihilation operator $a_{i}$ as the adjoint of the creation operator $\left(a_{i}^{\dagger}\right)^{\dagger}$. In this way, the annihilation operator annihilates an electron in the orbital $\chi_{i}$. 
We shall now analyze the situation in which the two operators act as an applied product in the same state, a Slater Determinant without the orbital $\chi_{i}$. Initially, it is considered a state $\left|\chi_{k} \ldots \chi_{l}\right\rangle$ which suffers the action of the operator $a_{i} a_{i}^{\dagger}+a_{i}^{\dagger} a_{i}$ as follows:

$$
\left(a_{i} a_{i}^{\dagger}+a_{i}^{\dagger} a_{i}\right)\left|\chi_{k} \ldots \chi_{l}\right\rangle=a_{i} a_{i}^{\dagger}\left|\chi_{k} \ldots \chi_{l}\right\rangle=a_{i}\left|\chi_{i} \chi_{k} \ldots \chi_{l}\right\rangle=\left|\chi_{k} \ldots \chi_{l}\right\rangle .
$$

On the other hand, if the state $\left|\chi_{i}\right\rangle$ is already occupied, then

$$
\begin{aligned}
\left(a_{i} a_{i}^{\dagger}+a_{i}^{\dagger} a_{i}\right)\left|\chi_{k} \ldots \chi_{i} \ldots \chi_{l}\right\rangle & =a_{i}^{\dagger} a_{i}\left|\chi_{k} \ldots \chi_{i} \ldots \chi_{l}\right\rangle=-a_{i}^{\dagger} a_{i}\left|\chi_{i} \ldots \chi_{k} \ldots \chi_{l}\right\rangle \\
& =-a_{i}^{\dagger}\left|\ldots \chi_{k} \ldots \chi_{l}\right\rangle=-\left|\chi_{i} \ldots \chi_{k} \ldots \chi_{l}\right\rangle \\
& =\left|\chi_{k} \ldots \chi_{i} \ldots \chi_{l}\right\rangle .
\end{aligned}
$$

In order that, in the Eqs. 2.38 and 2.39 there were obtained the same determinants. Thus, it is possible to write

$$
a_{i} a_{i}^{\dagger}+a_{i}^{\dagger} a_{i} \equiv\left\{a_{i}, a_{i}^{\dagger}\right\}=1
$$

Generally, it is considered the case in which the operator $a_{j} a_{i}^{\dagger}+a_{j}^{\dagger} a_{i}$ is applied for $i \neq j$. Unlike the above-mentioned case, the action of this operator on a generic Slater Determinant can only be not null if the $\chi_{i}$ orbital is occupied whereas the $\chi_{j}$ orbital remains empty. In this way,

$$
\begin{aligned}
\left(a_{i} a_{j}^{\dagger}+a_{j}^{\dagger} a_{i}\right)\left|\chi_{k} \ldots \chi_{i} \ldots \chi_{l}\right\rangle & =-\left(a_{i} a_{j}^{\dagger}+a_{j}^{\dagger} a_{i}\right)\left|\chi_{i} \ldots \chi_{k} \ldots \chi_{l}\right\rangle \\
& =-a_{i}\left|\chi_{j} \chi_{i} \ldots \chi_{k} \ldots \chi_{l}\right\rangle-a_{j}^{\dagger}\left|\ldots \chi_{k} \ldots \chi_{l}\right\rangle \\
& =a_{i}\left|\chi_{i} \chi_{j} \ldots \chi_{k} \ldots \chi_{l}\right\rangle-\left|\chi_{j} \ldots \chi_{k} \ldots \chi_{l}\right\rangle \\
& =\left|\chi_{j} \ldots \chi_{k} \ldots \chi_{l}\right\rangle-\left|\chi_{j} \ldots \chi_{k} \ldots \chi_{l}\right\rangle \\
& =0
\end{aligned}
$$

Therefore, it is possible to conclude that

$$
a_{i} a_{j}^{\dagger}+a_{j} a_{i}^{\dagger} \equiv\left\{a_{i}, a_{j}^{\dagger}\right\}=0
$$

if $i \neq j$. From the Eqs. 2.42 and 2.43, it is obtained that

$$
a_{i} a_{j}^{\dagger}+a_{j} a_{i}^{\dagger} \equiv\left\{a_{i}, a_{j}^{\dagger}\right\}=\delta_{i j}
$$

According to what was discussed before, the Slater Determinants can be represented using the creation and annihilation operators. Moreover, the anticommutation relations provide a representation by which the many-body wavefunction satisfies the 
antisymmetry principle. However, to a correct approach of the many-body theory without the use the determinants, the one- and two-electron operators $\left(O_{1}\right.$ and $O_{2}$ respectively) should be addressed by means of the creation and annihilation operators. Thereby, it is possible to express the matrix elements of the operators $O_{1}$ and $\mathrm{O}_{2}$ using only the algebraic properties of the creation and annihilation operators, so that $O_{1}$ and $O_{2}$ can be placed in the forms

$$
O_{1}=\sum_{i j}\langle i|h| j\rangle a_{i}^{\dagger} a_{j}
$$

and

$$
O_{2}=\sum_{i j k l}\langle i j|h| k l\rangle a_{i}^{\dagger} a_{j}^{\dagger} a_{k} a_{l}
$$

where

$$
\langle i j|h| k l\rangle=\left\langle i j\left|\frac{1}{r_{i j}}\right| k l\right\rangle .
$$

The operators $O_{1}$ and $O_{2}$ are independent of the number of electrons [65]. The advantage to use the second quantization formalism is due to the fact that this approach can treat, in the same way, sytems with different number of electrons, which is absolutely required when systems with a large number of particles, such as solids, are taken into account.

\subsection{The Tight-Binding Approach}

The translational invariance is a kind of discrete symmetry operation. Such symmetry possesses important applications in the solid state physics, i.e., for systems with spacial periodicity. Thereby, this consideration has proven to be fundamental to study conjugated polymers. The tight-binding approximation consists in considering a combination of the site atomic potentials as the potential of a crystalline lattice, i.e., it is assumes that these potentials are weakly superimposed [67]. In other words, this approximation adopts interactions between neighboring sites only. Considering onedimensional conductors, the tight-binding approximation may be performed through using the periodicity of the lattice. Such periodicity is, in fact, a discrete symmetry of the system named translational symmetry.

Generally, by considering a lattice with an one-dimensional periodic potential $a$, which $V(x \pm a)=V(x)$, it is possible to analyze the electronic motion in a lattice with equally spaced ions [67]. The translational operator, represented by $\tau(l)$, in which $l$ is arbitrary, has the following property

$$
\tau^{\dagger}(l) x \tau(l)=x+l .
$$


However, when $l$ coincides with the lattice spacing $a$, i. e., with the period of the potential, it should be noted that

$$
\tau^{\dagger}(l) V(x) \tau(l)=V(x+a)=V(x)
$$

Thus, due to the invariance under translations of the kinetic energy, for any displacement, and also to $\tau$ being an unitary operator, the Hamiltonian satisfies the equation

$$
\tau^{\dagger}(a) \mathcal{H} \tau(a)=\mathcal{H}
$$

that can be written as,

$$
[\mathcal{H}, \tau(a)]=0 .
$$

Therefore, the Hamiltonian and the translational operator can be diagonalized simultaneously [67]. Albeit the translational operator is unitary, it is not necessarily hermitian, and therefore it is expected its eigenvalue to be a complex number with unitary module.

It should now be discussed the case which the periodic potential is described by an infinity potential barrier between neighboring sites. Considering a state $|n\rangle$ given by $C_{n}^{\dagger}|\quad\rangle$, where $|n\rangle$ represents an electron localized in the $i$-th lattice site, it is possible to note that the wavefunction $\langle x \mid n\rangle$ is finite only inside the site $n$. This fact shows that $|n\rangle$ is an eigenstate with eigenvalue $E_{0}$, i. e., $\mathcal{H}|n\rangle=E_{0}|n\rangle$. Moreover, one can see that similar states, at some other site of the lattice, may possess the same energy $E_{0}$. Thus, a linear combination of eigenstates $|n\rangle$ is also an eigenstate of the Hamiltonian.

It is possible to note that $|n\rangle$ is not an eigenstate of the translational operator, due to the fact that when this operator is applied in $|n\rangle$ it is obtained that

$$
\tau(a)|n\rangle=|n+1\rangle
$$

Thereby, it becomes necessary to define an eigenstate that is, simultaneously, an eigenstate of $\mathcal{H}$ and $\tau(a)$. In this way, it is defined an eigenstate $|\theta\rangle$ that is a linear combination of eigenstates $|n\rangle$, which presents the following form

$$
|\theta\rangle=\sum_{n} e^{i \theta n}|n\rangle,
$$

where $\theta$ is a real parameter raging in the interval $[-\pi, \pi][67]$. Now, applying the translational operator $\tau(a)$ on $|\theta\rangle$, we arrive at

$$
\tau(a)|\theta\rangle=\sum_{n} e^{i \theta n} \tau(a)|n\rangle=\sum_{n} e^{i \theta n}|n+1\rangle=\sum_{n} e^{i \theta(n-1)}|n\rangle=e^{-i \theta}|\theta\rangle .
$$


Thus, considering an one-dimensional lattice with an infinity potential barrier between neighboring sites, the state $|\theta\rangle$ is an eigenstate of the translational operator $\tau(a)$ with eigenvalue $e^{-i \theta}$ and also eigenstate of the Hamiltonian operator having as eigenvalue $E_{0}$.

For real cases, i. e., when a finite potential barrier between neighboring sites is taken into account, the wavefunction $\langle x \mid \theta\rangle$ is not localized. In other words, the wavefunction $\left\langle x^{\prime} \mid \theta\right\rangle$ has its extremity extended through the other sites until the $\mathrm{n}$-th lattice site. The diagonal elements of $\mathcal{H}$ on the basis $|n\rangle$ are all the same due to the translational invariance, which means that

$$
\langle n|\mathcal{H}| n\rangle=E_{0}
$$

for any $n$. Assuming, for this moment, that the potential barriers between neighboring sites are high but not infinity, it is expected that the matrix elements of $\mathcal{H}$ between distant sites are null. Thus, it is assumed that only the outer elements of the diagonal are important, as shown in Eq. 2.55. In solid state physics, this affirmation is named Tight-Binding Approximation [67,68], where

$$
\left\langle n|\mathcal{H}| n^{\prime}\right\rangle=\left\{\begin{array}{l}
E_{0} \rightarrow n=n^{\prime} \\
-\Delta \rightarrow n=n^{\prime} \pm 1 \\
0 \rightarrow \forall n \neq n^{\prime}, \forall n \neq n^{\prime} \pm 1
\end{array}\right.
$$

Then, it is possible to write $\mathcal{H}$ as follows:

$$
\mathcal{H}=\sum_{n^{\prime}, n^{\prime \prime}}\left\langle n^{\prime}|\mathcal{H}| n^{\prime \prime}\right\rangle\left|n^{\prime}\right\rangle\left\langle n^{\prime \prime}\right|
$$

Therefore, the acting of $\mathcal{H}$ on the basis $\{|n\rangle\}$ results in

$$
\mathcal{H}|n\rangle=\sum_{n^{\prime}, n^{\prime \prime}}\left(\left\langle n^{\prime}|\mathcal{H}| n^{\prime \prime}\right)\left|n^{\prime}\right\rangle\left\langle n^{\prime \prime} \mid n\right\rangle=\sum_{n^{\prime}}\left(\left\langle n^{\prime}|\mathcal{H}| n\right)\left|n^{\prime}\right\rangle=E_{0}|n\rangle-\Delta|n+1\rangle-\Delta|n-1\rangle .\right.\right.
$$

One can see that $|n\rangle$ is not an eigenstate of $\mathcal{H}$. Now, acting $\mathcal{H}$ on $|\theta\rangle$, it is obtained that

$$
\mathcal{H}|\theta\rangle=\left(E_{0}-2 \Delta \cos \theta\right)|\theta\rangle .
$$

Thus, in summary, considering a lattice with finite potential barriers between the lattice sites, the basis set states $\{|\theta\rangle\}$ are degenerate and are eigenstates of the Hamiltonian operator with the eigenvalue ranging from $E_{0}-2 \Delta$ to $E_{0}+2 \Delta$ [67]. 


\subsection{The Langevin Equation}

The phenomenon that characterizes the motion of particles immersed in a certain fluid, moving under influence of random forces resulting from the collisions which are induced by thermal fluctuations is known as Brownian Motion [69]. In statistical mechanics, the Langevin Equation is defined as a stochastic differential equation that describes the brownian motion in a certain potential [70].

The simplest Langevin Equation is that which presents an constant potential. Thus, the brownian particle acceleration can be expressed is terms of its mass $m$ subjected to a viscous force, that is considered to be proportional to the velocity (Stokes Law), a noise term $\zeta(t)$ which is used in stochastic processes as a timedependent random variable that denotes the effect of several continuos collisions, and a force field $F(x)$ derived from intra and inter-molecular interactions. Thus, the Langevin Equation which describes this kind of system can be placed in the form [71]

$$
m \frac{d v(t)}{d t}=-m \gamma v(t)+F(x)+\zeta(t) .
$$

For a generic potential, the usual form of the Langevin Equation is written as

$$
m \frac{d v(t)}{d t}=-m \gamma v(t)+\zeta(t) .
$$

The random force $\zeta(t)$ has the following preperties: $\zeta(t)$ is a gaussian and stochastic noise with $\langle\zeta(t)\rangle=0$; the time-correlation between $\zeta(t)$ and $\zeta(0)$ is infinitely small, in order that $\langle\zeta(t) \zeta(0)\rangle=\beta \delta(t)$; and, finally, the particle motion is occasioned due to thermal bath fluctuations where $\langle v(0) \zeta(t)\rangle=0$ [71].

The generic solution to Eq. 2.60 may be obtained defining $v(t)=u(t) e^{-\gamma t}$, which $u(t)$ is a function to be determined. Thereby,

$$
\frac{d u}{d t}=e^{\gamma t} \zeta(t)
$$

which the solution is

$$
u=u_{0}+\int_{0}^{t} e^{\gamma t^{\prime}} \zeta\left(t^{\prime}\right) d t^{\prime}
$$

Thus,

$$
v=v_{0} e^{\gamma t^{\prime}}+e^{\gamma t^{\prime}} \int_{0}^{t} e^{\gamma t^{\prime}} \zeta\left(t^{\prime}\right) d t^{\prime}
$$

where $v_{0}$ is the particle's velocity at $t=0$. Such solution is valid for any function $\zeta(t)$. Using the above-mentioned noise and $\zeta(t)$ properties, it is possible to calculate the variance for the velocity

$$
\langle v\rangle=v_{0} e^{-\gamma t}
$$


and

$$
v-\langle v\rangle=e^{-\gamma t} \int_{0}^{t} e^{\gamma t^{\prime}} \zeta\left(t^{\prime}\right) d t^{\prime}
$$

Considering that $\langle v(0) \zeta(t)\rangle=0$, the Eq. 2.63 can be multiplied by $v(0)$, taking the average of all ensemble. Thus,

$$
\langle v(0) v(t)\rangle=\left\langle v^{2}(0)\right\rangle e^{-\gamma t}
$$

shows the loss of memory of the system regarding to its initial conditions and is consistent with the fact of considering the system to be markovian, i. e., a system in which the future event depends only on the immediately previous event [72]. Taking the square of the Eq. 2.63 and calculating, again, the average of all ensemble, considering the conditon $\langle\zeta(t) \zeta(0)\rangle=\beta \delta(t)$, we obtain

$$
\begin{aligned}
\left\langle v^{2}(t)\right\rangle & =\left\langle v^{2}(0)\right\rangle e^{-2 \gamma t}+\frac{e^{-2 \gamma t}}{m^{2}} \int_{0}^{t} \int_{0}^{t^{\prime}} e^{\gamma\left(t^{\prime}+t^{\prime \prime}\right)}\left\langle\zeta\left(t^{\prime}\right) \zeta\left(t^{\prime \prime}\right)\right\rangle d t^{\prime} d t^{\prime \prime} \\
& =\left\langle v^{2}(0)\right\rangle e^{-2 \gamma t}+\frac{\beta}{2 \gamma m^{2}}\left(1-e^{-2 \gamma t}\right) .
\end{aligned}
$$

For longer periods of time, i. e., in the stationary regime where $\langle v\rangle=0$, it is possible to obtain the $\beta$ value. Therefore, taking $t \rightarrow \infty$, we obtain

$$
\lim _{t \rightarrow \infty}\left\langle v^{2}(t)\right\rangle=\frac{\beta}{2 \gamma m^{2}}
$$

As it is known the energy equipartition theorem, where $(1 / 2) m\left\langle v^{2}(t)\right\rangle=(1 / 2) k_{B} T$. Thus, using this theorem, we can arrive at the following relation

$$
\beta=2 \gamma m K_{B} T
$$

Considering that the velocities distribution satisfies the Fokker-Plank equation [73],

$$
\frac{\partial}{\partial t} P(v, t)=\frac{\partial}{\partial v}\left(\gamma v+\frac{D_{v}}{2} \frac{\partial}{\partial v}\right) P(v, t)
$$

in which $D_{v}$ is related with the noise as follows

$$
D_{v}=\frac{1}{m^{2}} \int_{0}^{\infty}\langle\zeta(t) \zeta(0)\rangle d t=\frac{\beta}{m^{2}}
$$

Assuming, by hypothesis that $v(t)$ is a gaussian process, the stationary solution of the Eq. 2.70 assumes the form,

$$
P(v)=\left(\frac{m}{2 \pi K_{B} T}\right)^{\frac{1}{2}} e^{-\frac{m v^{2}}{2 K_{B} T}}
$$


Placing this equation in Eq. 2.70, is it possible to obtain that,

$$
D_{v}=\frac{2 K_{B} T}{m} \gamma=\frac{\beta}{m^{2}}
$$

and

$$
\gamma=\frac{1}{2 m K_{B} T} \int_{0}^{\infty}\langle\zeta(t) \zeta(0)\rangle d t
$$

where

$$
\langle\zeta(t) \zeta(0)\rangle=2 m \gamma K_{B} T \delta(t)
$$

which is the form of the Fluctuation-Dissipation theorem used in this work in order to include the temperature effects.

So far, we have introduced the theoretical formalism which is enough to discuss in detail the $\mathrm{Su}$-Schrieffer-Heeger model together with the modifications implemented. The next chapter presents this model, highlighting its fundamentals aspects. 


\section{The Su-Schrieffer-Heeger Model}

In this chapter, it is presented a version of the $\mathrm{Su}-\mathrm{Sch}$ rieffer-Heeger (SSH) model modified to include an external electric field, electron-electron interactions, a symmetrybreaking term, impurities and temperature effects. Particularly, a detailed treatment regarding the quasi-particle dynamics in organic semiconductors, from the framework of a tight-binding approach, is provided in order to better analyze the mechanisms involved on the generation and recombination dynamics of excited states in these materials. Moreover, an overview of the quasi-particles (as agents as well as products) included in the aforementioned mechanisms is provided in order to clarify the understanding of the results discussed in the papers that constitute this thesis.

\subsection{The SSH-type Hamiltonian}

An extremely convenient treatment of the conductivity in polyacetylene molecules was first presented in pioneering papers by Su, Schrieffer, and Heeger in 1979 [74-76]. The proposed model showed that the conductivity in this material is related to topological defects in its structure, which could arise easily when a sample of the material is exposed to doping agents $[10,77,78]$. Furthermore, it was shown that these charged defects could move through the lattice when an external electric field was applied.

Concerning the $\pi$-electrons theory, it is known that for an ion (a $\mathrm{CH}$ group for the polyacetylene) there exists six degrees of freedom per unity cell [32]. For the treatment of a polyacetylene molecule, in the scope of the SSH model, an important simplification is introduced: it is considered (from the electronic point of view) only the normal vibration modes that couple, predominantly, the $\pi$-electrons [76]. For the polyacetylene, this degree of freedom denotes the bond between the carbon atoms which can stretch and shrink alternately. Thus, projecting the ionic coordinates in a horizontal axis that extends through the lattice, the problem becomes onedimensional, according represented in Figure 3.1. 


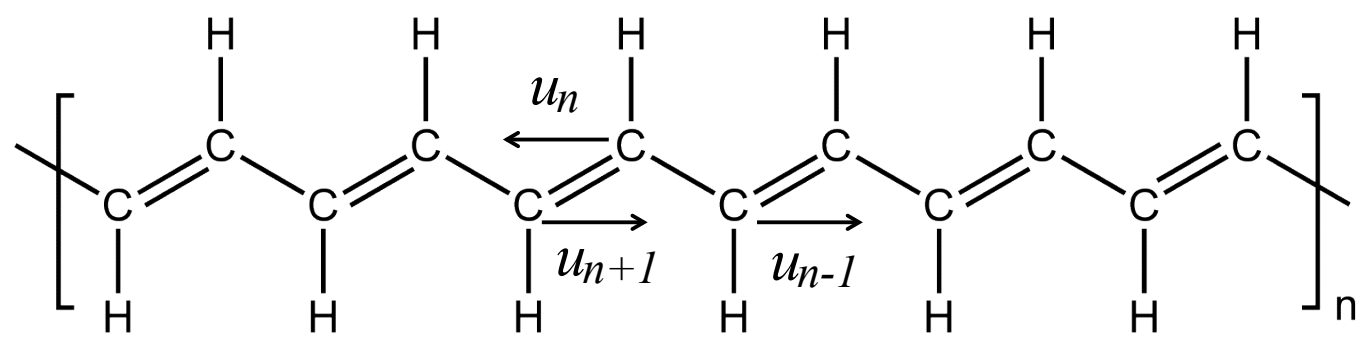

Figure 3.1: Schematic representation of a dimerized trans-polyacetylene molecule with its displacement coordinate $u$, which denotes the unique degree of freedom.

For the polyacetylene molecule, the $\sigma$-electrons represent the strong covalent bonds between carbon-carbon and carbon-hydrogen atoms, of approximately $3 \mathrm{eV}$ $[79,80]$. This type of bond is the main responsible for the lattice backbone structure of polyacetylene. The $\pi$-electrons, in their turn, tend to form less localized bonds, of about $1 \mathrm{eV}$ [80]. These bonds are formed by the overlap of two adjacent atomic orbitals $\left(2 \mathrm{P}_{z}\right)$ which are perpendicular to the plane of the polyacetylene molecule. Moreover, the $\pi$-bonds are considered weak if compared to the $\sigma$-bonds. The $\pi-$ bonds are responsible for the alternation between the single and double bonds, i. e., these bonds are responsible by the dimers formation (the lattice dimerization) [79].

The $u_{n}$ coordinates, as shown in Figure 3.1, are the displacements of the $\mathrm{CH}$ groups projected in the $x$-axis for the case where the lattice is not dimerized [74]. Considering the ground state, which is dimerized, if $u_{n}>0$ then, necessarily, $u_{n}+1<0$. It occurs due to the consideration of the tigh-binding approach (where only the first next neighbors can interact), as mentioned in the previous chapter, and also by considering that the system is one-dimensional, i. e., the coordinate $u$ is the only necessary to describe the system [75]. For a dimerized lattice, $\left|u_{n+1}-u_{n}\right| \cong 0.08 \AA$ and the lattice parameter $a=1.40 \times \sqrt{3} / 2 \AA \cong 1.22 \AA[75]$.

In summary, the SSH model is an extension of the tight-binding approach. Thus, the interchain interaction is neglected (one-dimensional system). Moreover, the coupling between the $\sigma$ - and $\pi$-electrons is also neglected. The $\mathrm{CH}$ group, which has six degrees of freedom, is described by only its translations in the lattice direction by means of the $u$ coordinate [32]. The other five degrees of freedom are ignored, since that in a first order approximation they are not related to the lattice dimerization [32]. Finally, the coupling between the $\pi$-electrons is considered in the scope of a mean-field approximation, i. e., the electronic correlation effects are not considered. In the basis of the atomic orbitals, for the $\pi$-electrons, the matrix elements are unknown, except for the first neighbors [76]. 
From the introduction about some important features discussed just above, we now can define the SSH Hamiltonian. In order to do so, it is initially considered that the displacements $u_{n}$ are smaller than the bond between the carbon atoms, $u_{n} \cong 0.04 \AA$. The potential energy of the $\sigma$-electrons can be expanded in a Taylor series truncated in second order as follows

$$
E_{\sigma}=E_{\sigma}(0)+\sum_{n} \frac{\partial E_{\sigma}}{\partial\left(u_{n+1}-u_{n}\right)}\left(u_{n+1}-u_{n}\right)+\sum_{n} \frac{1}{2 !} \frac{\partial^{2} E_{\sigma}}{\partial\left(u_{n+1}-u_{n}\right)^{2}}\left(u_{n+1}-u_{n}\right)^{2}+\ldots
$$

One can see that the first term of the expansion is a constant. Thereby, it can be defined as zero. The second term is also null, once the expansion is carried out considering a point that has vanishing first derivative. Due to the symmetry shown by the coordinates $u_{n}$, the coefficient of the second order terms could be defined as equal to a constant $K$ [32]. Thus, approximating this potential to a harmonic oscillator potential, it can be written as

$$
\frac{1}{2} \sum_{n} K\left(u_{n+1}-u_{n}\right)^{2}
$$

The $\pi$-electrons, in their turn, are treated by the hopping term, in an expansion of the tight-binding approximation which considers coupling between the electronic and lattice parts of the system. They are described by an hopping integral according to the following equation

$$
t_{n+1, n}=t_{0}-\alpha\left(u_{n+1}-u_{n}\right),
$$

where $t_{0}$ denotes the hopping integral of a $\pi$-electron between neighboring sites in an evenly spaced lattice and $\alpha$ is the electron-phonon coupling constant [76,81-83]. Due to the fact that the bond lengths can not suffer larger variations, the first order expansion to the hopping integral is a reasonable approximation.

The kinetic energy of the sites, i. e., $\mathrm{CH}$ groups, is written as

$$
E=\frac{1}{2} \sum_{n} M \dot{u}_{n}^{2}
$$

where $M$ is their masses. Therefore, the SSH Hamiltonian, written in the second quantization formalism, assumes the form

$$
H=-\sum_{n, s}\left(t_{n, n+1} C_{n+1, s}^{\dagger} C_{n, s}+h . c\right)+\frac{1}{2} \sum_{n} K\left(u_{n+1}-u_{n}^{2}\right)+\sum_{n} \frac{p_{n}^{2}}{2 M},
$$

where $n$ indexes the sites of the chain $[74,75]$. The operator $C_{n, s}^{\dagger}\left(C_{n, s}\right)$ creates (annihilates) a $\pi$-electron state at the nth site with spin $s$ [74]. 


\subsection{The Dimerized Lattice}

The dimerized lattice is the simplest model to a semiconducting polymer and, particularly, characterizes the trans-polyacetylene structure. A semiconducting polymer chain is usually extensive and may present around 3000 sites. In the SSH model, such system can be represented by a $\mathrm{N}$-size finite lattice with periodic boundary conditions. The stationary solution for a perfectly dimerized lattice was firstly investigated by $\mathrm{Su}$, Schrieffer, and Heeger in the pioneering work which describes the SSH model [75]. In this solution, the term that describes the kinetic energy of the $\mathrm{CH}$ groups is naturally null. In order to describe this system, initially it is considered that a dimerized lattice can be treated in the framework of the Born-Oppenheimer approximation [75], where the $u_{n}$ coordinates has the form

$$
u_{n}=(-1)^{n} u
$$

which make the lattice perfectly dimerized by considering $u$ as a constant. Rewriting the hopping term, given by the Eq. 3.3, it is obtained that

$$
t_{n, n+1}=t_{0}+2 \alpha u(-1)^{n}
$$

Thus, the Hamiltonian can be written as

$$
H(u)=-\sum\left(t_{0}+2 \alpha u(-1)^{n}\right)\left[C_{n+1, s}^{\dagger} C_{n, s}+C_{n, s}^{\dagger} C_{n+1, s}\right]+2 K N u^{2} .
$$

Is it possible to diagonalize analytically such Hamiltonian to obtain the electronic spectrum. In order to do so, the following transforms are used [75]

$$
\begin{aligned}
C_{k s}^{c \dagger} & =\frac{-i}{\sqrt{N}} \sum_{n}(-1)^{n} e^{-i k n a} C_{n s}^{\dagger}, \\
C_{k s}^{v \dagger} & =\frac{1}{\sqrt{N}} \sum_{n} e^{-i k n a} C_{n s}^{\dagger} .
\end{aligned}
$$

Such transforms describe the electronic spectrum at the first Brillouin zone, $\pi / 2 a \leq$ $k<-\pi / 2 a$. Thus,

$$
C_{n, s}=\frac{1}{\sqrt{N}} \sum_{k}\left[e^{-i k a n}\left(C_{k s}^{v}+i(-1)^{n} C_{k s}^{c}\right)\right]
$$

Performing the sum for the index $n$, taking into consideration that

$$
\frac{1}{N} \sum_{n} e^{i a n\left(k-k^{\prime}\right)}=\delta_{k k^{\prime}}
$$


and

$$
\sum_{n}(-1)^{n} e^{i a n\left(k-k^{\prime}\right)}=\sum_{n} e^{i a n\left(k-k^{\prime}+\pi / a\right)},
$$

the Hamiltonian can assume the form

$$
\begin{aligned}
H^{d}(u) & =\sum_{k s}\left[2 t_{0} \cos (k a)\left(C_{k s}^{c \dagger} C_{k s}^{c}-C_{k s}^{v \dagger} C_{k s}^{v}\right)\right. \\
& \left.+4 \alpha \operatorname{sen}(k a)\left(C_{k s}^{c \dagger} C_{k s}^{v}+C_{k s}^{v \dagger} C_{k s}^{c}\right)\right]+2 N K u^{2} .
\end{aligned}
$$

Finally, in order to represent $H^{d}(u)$ in the diagonal form, it is important to define the new operators $a_{k s}^{c}$ and $a_{k s}^{v}$ that can be written as follows [75]

$$
\left(\begin{array}{l}
a_{k s}^{v} \\
a_{k s}^{c}
\end{array}\right)=\left(\begin{array}{cc}
\alpha_{k} & -\beta_{k} \\
\beta_{k}^{*} & \alpha_{k}^{*}
\end{array}\right)\left(\begin{array}{l}
c_{k s}^{v} \\
c_{k s}^{c}
\end{array}\right)
$$

where

$$
\left|\alpha_{k}\right|^{2}+\left|\beta_{k}\right|^{2}=1
$$

By calculating the parameters $\alpha_{k}$ and $\beta_{k}$ which diagonalize the Hamiltonian, we obtain

$$
\alpha_{k}=\left[\frac{1}{2}\left(1+\frac{\epsilon_{k}}{E_{k}}\right)\right]^{\frac{1}{2}}
$$

and

$$
\beta_{k}=\left[\frac{1}{2}\left(1-\frac{\epsilon_{k}}{E_{k}}\right)\right]^{\frac{1}{2}} \operatorname{signal}(k),
$$

where

$$
\begin{gathered}
\epsilon_{k}=2 t_{0} \cos (k a), \\
\Delta_{k}=4 \alpha u \sin (k a),
\end{gathered}
$$

and

$$
E_{k}=\sqrt{\epsilon_{k}^{2}+\Delta_{k}^{2}}
$$

Thus, the Hamiltonian becomes diagonal in the representation of the operators $a_{k s}^{c}$ and $a_{k s}^{v}$

$$
H^{d}(u)=\sum_{k s} E_{k}\left(a_{k s}^{c \dagger} a_{k s}^{c}-a_{k s}^{v \dagger} a_{k s}^{v}\right)+2 N K u^{2} .
$$

In this equation, $E_{k}>0$ represents the energy values for the conduction band, whereas $E_{k}<0$ represents the valence band energies. Therefore, the gap is given by 
the difference between the energies of the conduction and valence bands presenting the value of $8 \alpha u$ for the Fermi wave vector $\vec{k}_{F}=\pi / 2 a$. The ground state energy is given by the sum over all the occupied states

$$
\begin{aligned}
E_{0}(u) & =\sum_{k s}{ }^{\prime} E_{k}+2 N K u^{2} \\
& =\sum_{k s} ' \sqrt{\left(2 t_{0} \cos (k a)\right)^{2}+(4 \alpha u \operatorname{sen}(k a))^{2}}+2 N K u^{2},
\end{aligned}
$$

where the prime represents the sum over occupied states only. Considering that the system has a large number of particles, it is possible to approximate the discrete energy in a continuous form as

$$
\begin{aligned}
E_{0}(u) & =\frac{-2 L}{\pi} \int_{0}^{\frac{\pi}{2 a}} \sqrt{\left(2 t_{0} \cos (k a)\right)^{2}+(4 \alpha u \operatorname{sen}(k a))^{2}} d k+2 N K u^{2} \\
& =\frac{4 N t_{0}}{\pi} E\left(1-z^{2}\right)+\frac{N K t_{0}^{2} z^{2}}{2 \alpha^{2}} .
\end{aligned}
$$

In this equation, $E\left(1-z^{2}\right)$ is an elliptic integral of second specie, with $z=2 \alpha u / t_{0}$, and $L=N a$, the lattice length. The expansion of the elliptic integral for a small value for $z$ can be written as,

$$
E_{0}(z)=\frac{-4 N t_{0}}{\pi}\left[1+\frac{1}{2}\left(\frac{\ln (4)}{|z|}-\frac{1}{2}\right) z^{2}+\ldots\right]+\frac{N K t_{0}^{2} z^{2}}{2 \alpha^{2}} .
$$

The energy $E_{0}(u)$ has a local maximum for $u=0$ (which denotes an undimerized lattice). Truncating the expansion of the elliptic integral, for a small value of $z$, we can obtain an $u_{0}$ value which minimizes the energy. It is also possible to write the density of states per spin to the perfectly dimerized lattice as,

$$
\rho(E)=\frac{L}{2 \pi\left|\frac{d E_{k}}{d_{k}}\right|}=\left\{\begin{array}{ccc}
\frac{N}{\pi} \frac{|E|}{\sqrt{\left(4 t_{0}-E^{2}\right)\left(E^{2}-\Delta_{0}^{2}\right)}} & \text { se } & \Delta_{0} \leq|E| \leq 2 t_{0}, \\
0 & \text { for the other cases. }
\end{array}\right.
$$

Related to the ground state degeneracy, there exists an elementary excitation which corresponds to a domain wall (termed soliton) [74-76]. The equations presented so far in this chapter summarize the theory of the SSH model. The original work presents a stationary solution for a perfectly dimerized lattice and, also, the formulation of the solitons theory for polyacetylene lattices. This model is the most simple to describe the conductivity in polyacetylene lattices. However, this model should be extended in order to analyze the charge carrier dynamics in these materials under influence of an external electric field, Coulomb interactions, impurities, and temperature effects. The following sections are devoted to explain how such effects are taken into account in our model. 


\subsection{The Electric Field and the Symmetry Breaking Term}

The first modification implemented in the standard SSH model was made in order to include the effects of an external electric field. The field is included in the Hamiltonian as a vector potential A modifying the Eq. 3.3 as follows,

$$
t_{n, n+1}=e^{-i \gamma \mathbf{A}(t)}\left[t_{0}-\alpha y_{n}\right]
$$

where $y_{n}$ is defined as $y_{n}=u_{n+1}-u_{n} \cdot \gamma=e a / \hbar c$, with $e$ being the absolute value of the electronic charge, $a$ is the lattice constant, and $c$ is the speed of light [84-87]. The relation between the time-dependent vector potential $\mathbf{A}$ and the uniform electric field $\mathbf{E}$ is given by

$$
\mathbf{E}(t)=-\frac{1}{c} \dot{\mathbf{A}}(t)
$$

The implementation of the electric field through the potential vector is convenient due to the fact that the system has periodic boundary conditions. Another point that is worthy of attention is that the abrupt inclusion of the electric field in the simulations may cause numerical errors. In many cases the charge carriers could be artificially annihilated in a few femtoseconds. Such problem is solved by turning on the field adiabatically according to the following equations

$$
\mathbf{A}(t)=\left\{\begin{array}{cll}
0 & \text { if } & t<0, \\
\frac{-1}{2} c E\left[t-\frac{\tau}{\pi} \operatorname{sen}\left(\frac{\pi t}{\tau}\right)\right] & \text { if } & 0 \leq t<\tau, \\
-c\left(t-\frac{\tau}{2}\right) & \text { if } & \tau \leq t<t_{f}, \\
-c E t_{f} & \text { if } & t_{f} \leq t<t_{f}+\tau, \\
\left.\frac{-1}{2} c E\left[t+t_{f}-\tau+\frac{\tau}{\pi} \operatorname{sen}\left(t-t_{f}+\pi\right)\right)\right] & \text { if } & t \geq \tau .
\end{array}\right.
$$

where the parameters $\tau$ represents the time period for which the field acts on the system, whereas $t_{f}$ denotes the time where the field is at its maximum.

In order to consider cis-polyacetylene chains, it should be included a symmetry breaking term in the hopping integral $t_{n+1, n}$, which is named Brazovskii-Kirova symmetry breaking term, represented here as $\delta_{0}$. To include this term in the model, Eq. 3.26 should be rewritten as follows [86, 88, 89],

$$
t_{n, n+1}=e^{-i \gamma \mathbf{A}(t)}\left[\left(1+(-1)^{n} \delta_{0}\right) t_{0}-\alpha y_{n}\right]
$$




\subsection{Electron-Electron Interactions and Impurity Ef- fects}

The doping processes in conjugated polymers are usually not performed by replacing atoms in the materials for metals or semimetals dopants, as in the case of inorganic semiconductors. In organic semiconductors, such processes are carried out by means sorption, i. e., the impurity is localized closer to the conjugated polymer lattice and the interaction between them affects only the eigen energies of the lattice site where it is adjacent. Consequently, this kind of doping process does not affect the nature of the bonds. In order to taken into account the impurity effects, a new term $H_{i m p}$ should be added to the SSH Hamiltonian presented in Eq. 3.5, henceforward named $H_{S S H}$. The new overall Hamiltonian $\left(H_{\text {total }}\right)$ assumes the form $H_{\text {total }}=H_{S S H}+H_{\text {imp }}$, with the last term denoting the inclusion of impurity effects in a particular site of the lattice. This term is written as

$$
H_{i m p}=I_{j} C_{j, s}^{\dagger} C_{j, s}
$$

The electron-electron interactions are descried by the extended Hubbard Model. In this way, the overall Hamiltonian has now the form $H_{\text {total }}=H_{S S H}+H_{i m p}+H_{e-e}$, where the last term is written as [90-92]

$$
H_{e e}=U \sum_{i}\left(C_{i, \uparrow}^{\dagger} C_{i, \uparrow}-\frac{1}{2}\right)\left(C_{i, \downarrow}^{\dagger} C_{i, \downarrow}-\frac{1}{2}\right)+V \sum_{i}\left(n_{i}-1\right)\left(n_{i+1}-1\right),
$$

where $n_{i}=C_{i, \uparrow}^{\dagger} C_{i, \uparrow}+C_{i, \downarrow}^{\dagger} C_{i, \downarrow}, U$ is the onsite electron-electron coulombian interaction, and $V$ is the neighboring sites electron-electron interactions. It should be noted that the present form of the Hubbard interactions maintain the electron-hole symmetry of the system.

\subsection{The System Dynamics}

In order to perform the time evolution of the system, initially it is considered a selfconsistent state regarding to the degrees of freedom for electrons and phonons. This point will be further discussed latter. After that, the system dynamics is performed in the framework of the Ehrenfest Molecular Dynamics [86]. The lattice backbone dynamics is carried out in a classical approach by means of the Euler-Lagrange equations

$$
\frac{d}{d t}\left(\frac{\partial\langle L\rangle}{\partial \dot{u}_{n}}\right)-\frac{\partial\langle L\rangle}{\partial u_{n}}=0
$$


The expectation value for the Lagrangian can be obtained from the terms of the Hamiltonian Eq. 3.5. Thus,

$$
\begin{aligned}
\langle L\rangle & =\langle T\rangle-\langle V\rangle \\
& =\sum_{n} \frac{M}{2} \dot{u}_{n}^{2}\langle\psi \mid \psi\rangle-\sum_{n} \frac{K}{2}\left(u_{n+1}-u_{n}\right)\langle\psi \mid \psi\rangle \\
& -\sum_{n s}\left[t_{0}-\alpha\left(u_{n+1}-u_{n}\right)\right]\left\langle\psi\left|\left(C_{n+1, s}^{\dagger} C_{n, s}+C_{n s}^{\dagger} C_{n+1, s}\right)\right| \psi\right\rangle,
\end{aligned}
$$

where the last term represents the expectation value to the electronic Hamiltonian.

The representation of the expectation value of the electronic Hamiltonian can be written in terms of

$$
B_{n, n^{\prime}} \equiv \sum_{k s}{ }^{\prime} \psi_{k s}^{*}(n, t) \psi_{k s}\left(n^{\prime}, t\right)
$$

as

$$
\left\langle H_{e}\right\rangle=-\sum^{n}\left[t_{0}-\alpha\left(u_{n+1}-u_{n}\right)\right]\left(B_{n, n+1}+B_{n, n+1}^{*}\right) .
$$

This permits to rewrite the expected value to the Lagrangian as,

$$
\begin{aligned}
\langle L\rangle & =\sum_{n} \frac{M}{2} \dot{u}_{n}^{2}-\sum_{n} \frac{K}{2}\left(u_{n+1}-u_{n}\right) \\
& +\sum_{n}\left[t_{0}-\alpha\left(u_{n+1}-u_{n}\right)\right]\left(B_{n, n+1}+B_{n, n+1}^{*}\right) .
\end{aligned}
$$

The lattice dynamics is given by the solution of Eq. 3.32 for the expectation value of the Lagrangian $\langle L\rangle$ expressed in Eq. 3.36. From this, we obtain [81]

$$
M \ddot{u}_{n}=F_{n}(t),
$$

where

$$
\begin{aligned}
F_{n}(t) & =-K\left[2 u_{n}(t)-u_{n+1}(t)-u_{n-1}(t)\right] \\
& +\alpha\left[\left(B_{n, n+1}+B_{n-1, n}\right)+\left(B_{n+1, n}+B_{n, n-1}\right)\right] .
\end{aligned}
$$

Using the definition of the time-derivative for $u_{n}$, i. e.,

$$
\dot{u}=\frac{u_{n}(t+d t)-u_{n}(t)}{d t},
$$

we can integrate the equations of motion using the Verlet Algorithm [86,87]

$$
u_{n}(t+d t)=u_{n}(t)+\dot{u}_{n}(t) d t
$$


and

$$
\dot{u}_{n}(t+d t)=\dot{u}_{n}(t)+\frac{F_{n}(t)}{M} d t .
$$

In its turn, the electronic dynamics is governed by the time-dependent Schrödinger equation,

$$
i \hbar \frac{\partial \psi_{k}}{\partial t}=H_{e} \psi_{k}
$$

which can be formally solved using

$$
\psi_{k}(t)=\exp \left[\int_{0}^{t} \frac{H_{e}\left(t^{\prime}\right)}{\hbar} d t^{\prime}\right] \psi_{k}(0)
$$

where $H_{e}\left(t^{\prime}\right)$ is the electronic Hamiltonian at a given time $t^{\prime}$. Especifically,

$$
\psi_{k}(t+d t)=\exp \left[\frac{-i}{\hbar} H_{e}(t) d t\right] \psi_{k}(t)
$$

We can expand $\psi_{k}(t)$ as follows,

$$
\psi_{k}(t)=\sum_{l} C_{l k} \phi_{l}(t)
$$

where $C_{l k}=\left\langle\phi_{l} \mid \psi_{k}\right\rangle$, with $\left\{\phi_{l}\right\}$ and $\left\{\varepsilon_{l}\right\}$ being the eigenfunctions and eigenvalues of the electronic Hamiltonian at a given time $t$. In this way, placing $\psi_{k}(t)$ in Eq. 3.44, we obtain [81]

$$
\psi_{n, k}(t+d t)=\sum_{l}\left[\sum_{m} \phi^{*}(m, t) \psi_{k}(m, t)\right] \exp \left[\frac{-i}{\hbar} \varepsilon_{l} d t\right] \phi_{l}(n, t) .
$$

Thus, by knowing the set of eigenstates $\left\{\psi_{k}\right\}$ at a given time $t$, it is possible to calculate $\left\{\psi_{k}\right\}$ at $t+d t$.

We now turn our attention to the preparation of the initial self-consistent state regarding the degrees of freedom of electrons and phonons. For the construction of the initial state (stationary state), where $d u_{n} / d t=0$, the Lagrangian can be written as

$$
\begin{aligned}
\langle L\rangle= & -\sum_{n} \frac{K}{2}\left(u_{n+1}-u_{n}\right)^{2}\langle\psi \mid \psi\rangle \\
& +\sum_{n s}\left[t_{0}-\alpha\left(u_{n+1}-u_{n}\right)\right]\left\langle\psi\left|\left(C_{n+1, s}^{\dagger} C_{n, s}+C_{n s}^{\dagger} C_{n+1, s}\right)\right| \psi\right\rangle .
\end{aligned}
$$

Using the definition $y_{n} \equiv u_{n+1}-u_{n}$, and Eq. 3.34, we have

$$
\langle L\rangle=-\sum_{n} \frac{K}{2} y_{n}^{2}+\sum_{n s}\left(t_{0}-\alpha_{n}\right)\left(B_{n+1, n}+B_{n+1, n}^{*}\right) .
$$


For this case, the Euler-Lagrange equations are written as

$$
\frac{\partial\langle L\rangle}{\partial y_{n}}=0
$$

which leads to

$$
y_{n}=-\frac{\alpha}{K}\left(B_{n, n+1}+B_{n, n+1}^{*}\right) .
$$

However, due to the fact that the system has periodic boundary conditions, the following condition should be satisfied

$$
\sum_{n} y_{n}=0
$$

Therefore, it is necessary to add one term in Eq. 3.50 as follows

$$
y_{n}=-\frac{\alpha}{K}\left(B_{n, n+1}+B_{n, n+1}^{*}\right)+\frac{\alpha}{N K}\left[\sum_{n}\left(B_{n, n+1}+B_{n, n+1}^{*}\right)\right] .
$$

In summary, in order to solve these equations numerically, first a stationary state that is self-consistent with all degrees of freedom of the system (lattice and electrons) needs to be obtained. The initial bond configuration and the electronic structure of a polymer chain containing a quasi-particle can be achieved by solving the following self-consistent equations of the bond configuration $\left\{u_{n}\right\}$ and the electronic wavefunctions $\left\{\phi_{n}\right\}[36]$ :

$$
\begin{gathered}
u_{n-1}-u_{n}=-\frac{2 \alpha}{K} \sum_{\mu} \phi_{\mu}(n) \phi_{\mu}(n+1)+\frac{2 \alpha}{N K} \sum_{\mu, n} \phi_{\mu}(n) \phi_{\mu}(n+1), \\
\varepsilon_{\mu} \phi_{\mu}(n)=-\left[t_{0}-\alpha\left(u_{n-1}-u_{n}\right)\right] \phi_{\mu}(n+1)-\left[t_{0}-\alpha\left(u_{n}-u_{n-1}\right)\right] \phi_{\mu}(n-1)
\end{gathered}
$$

where $\varepsilon_{\mu}$ is the eigenvalue of the $\mu$-th energy level. We begin by constructing the Hamiltonian from a $\left\{y_{n}\right\}$ set of conveniently closer positions. By solving the timeindependent Scrhödinger equation

$$
H_{\text {ele }}\left|\psi_{k}\right\rangle=E_{\text {ele }}\left|\psi_{k}\right\rangle,
$$

a new set of eingenfunctions $\left\{\psi_{k}\right\}$ is obtained, and from that a new set of coordinates $\left\{y_{n}\right\}$ using Eq. 3.53. Iterative repetitions of this procedure yields a self-consistent initial state when $\left\{y_{n}\right\}$ is close enough to the previous step. After that, from the self-consistent initial state, we can evolve the system determining the eigenstates and eigenvalues of the electronic hamiltonian at each step. The equations 3.40, 3.41, and 3.46 govern the system dynamics. 


\subsection{Temperature Effects and Occupation Number}

In order to take into account the temperature effects, the SSH model should be further modified. The temperature is included in the equations that describe the lattice backbone dynamics (classical part of the Hamiltonian). Hence, the temperature influence on the electronic part of the system is only considered indirectly by means of coupling terms, through the term expressed in Eq. 3.34. It should be mentioned, however, that the contribution of the electronic part to thermal properties is usually considered much smaller than that of the cores.

The temperature effects on the lattice are simulated using the Langevin Equation, as mentioned in the previous chapter. Here, it is used the stochastic signal $\zeta_{n}(t)$, generally known as "white noise". Such nomenclature is due to the fact that the spectral intensity of a signal is defined as a Fourier transform of the eigen-correlation function. The noise $\zeta_{n}(t)$ adopted in this work has as the correlation function a Dirac delta function. As the Fourier transform of a delta function is constant, it is understood that all the frequencies are present with similar intensity, thus the analogy with the white light.

Here, the temperature effects are simulated by adding thermal gaussian random forces with zero mean value $\left\langle\zeta_{n}(t)\right\rangle \equiv 0$ and variance $\left\langle\zeta_{n}(t) \zeta_{n}\left(t^{\prime}\right)\right\rangle=2 k_{B} T \gamma M \delta\left(t-t^{\prime}\right)$ $[71,93]$. We adopted a white stochastic signal $\zeta_{n}(t)$ as the fluctuation term. Also, in order to keep the temperature constant at its initial value after a transient period (named thermalization), it is necessary to introduce a damping factor, $\gamma$. Therefore, Eq. (3.37) is modified to

$$
M \ddot{u}=-\gamma \dot{u}+\zeta_{n}(t)+F_{n}(t) .
$$

The modified equations no longer defines a set of ordinary differential equations (ODEs); rather, in this formalism we deal with a set of stochastical differential equations (SDEs). Therefore, it is important to find a proper integrator for solving SDEs. Since our model assumes a classical treatment for the lattice, it is possible to use the regular Langevin-type approach to take thermal effects into account. Several discretizations technics for the modified equation have been suggested by the literature. We have used the velocity-verlet (Eqs. 3.41 and 3.46) that is very similar to the popular BBK integrator [93]. Furthermore, we have introduced both the dissipative force and the gaussian random force in such a way to possess the power spectral density given by the fluctuation-dissipation theorem. In this way, the fluctuations can be 
obtained by using

$$
\zeta_{n}(t)=\sqrt{\left(2 k_{B} T \gamma M\right) / \Delta t} \times Z^{n},
$$

where $Z^{n}$ is a random number [93]. The damping constant can be determined by low temperature lattice thermal conductivity measurements. The $\gamma$ value used here has the same order of magnitude as expected from experimental data of Raman spectral line width in polydiacetylene $\left(\gamma=0.01 \omega_{Q}\right)$ [71]. It should be emphasized that this procedure of including temperature effects by means of a Langevin formalism has been extensively used in the literature and is known to yield excellent qualitative results.

As discussed above, the electronic wave functions and the lattice displacements at the $(j+1)$ th time step are obtained from the $j$ th time step. At time $t_{j}$ the wavefunctions $\left\{\psi_{k, s}\left(i, t_{j}\right)\right\}$ are expressed as an expansion of the eigenfunctions $\left\{\phi_{l, s}\right\}$ of the electronic Hamiltonian at that moment:

$$
\psi_{k, s}\left(i, t_{j}\right)=\sum_{l=1}^{N} C_{l, k}^{s} \phi_{l, s}(i),
$$

where $C_{l, k}^{s}$ are the expansion coefficients. The occupation number for each eigenstate $\phi_{l, s}$ is

$$
\eta_{l, s}\left(t_{j}\right)=\sum_{k}{ }^{\prime}\left|C_{l, k}^{s}\left(t_{j}\right)\right|^{2}
$$

$\eta_{l, s}\left(t_{j}\right)$ contains information concerning the redistribution of electrons among the energy levels, thus being of fundamental importance to analyze the products formed after the recombination mechanism as well as their yields.

\subsection{Charge Carriers and Excited States}

Due to the diversity of experimental results regarding the conductivity in conjugated polymers, a need to propose different conduction mechanisms from those used to describe the charge transport in inorganic materials arises naturally. As mentioned in the first chapter, the first conduction model in organic semiconductors was proposed by $\mathrm{Su}$, Schrieffer and Heeger (the SSH model). In this model, the organic semiconductor is formed by long chains, for which the occurrence of topological defects in the polymerization process becomes natural. These structural defects could also be created upon doping or photoexcitation processes forming radicals. These defects may change the dimerization shape generating specific forms to the $\mathrm{CH}$ groups configuration. The high conductivity of the conjugated polymers is due to the presence 
of these defects in the lattice. When an external electric field is applied, if the defect has charge, it can move through the lattice. Thus, these defects behave as charge carriers. This collective behavior of the lattice and electronic system is characterized as quasi-particles.

The conventional charge carrier in organics semiconductors par excellence is the polaron [55,94-100]. This quasi-particle can be understood as a rearrangement of the $\pi$-electrons, which polarizes the lattice locally, resulting in a short range modification to the spacial configuration of the carbon atoms. In organic semiconductors, the polaron could also be understood as a bond state of solitons (the original solution for the PA chain, previously mentioned) pair. In other words, in organic conductors, the polaron is a quasi-particle arising from the electron-phonon interactions manifested in the form of a lattice distortion.

Regarding its electronic structure, the topological defects represents a symmetry breaking in the lattice. As a consequence, one electronic state arises in the band gap. The electronic spectrum of the polaron presents two energy levels inside the band gap: one is closer to the conduction band whereas the other one closer to the valence band, as shown in Figure 3.2. A polaron has spin $\pm 1 / 2$ and a charge $\pm e$. Thus, polarons can respond, simultaneously, to the action of electrical and magnetic fields.

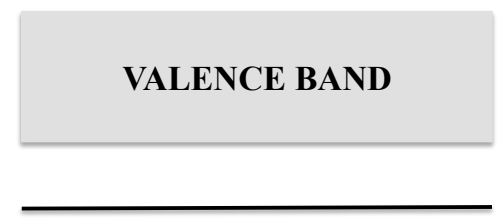

(a)
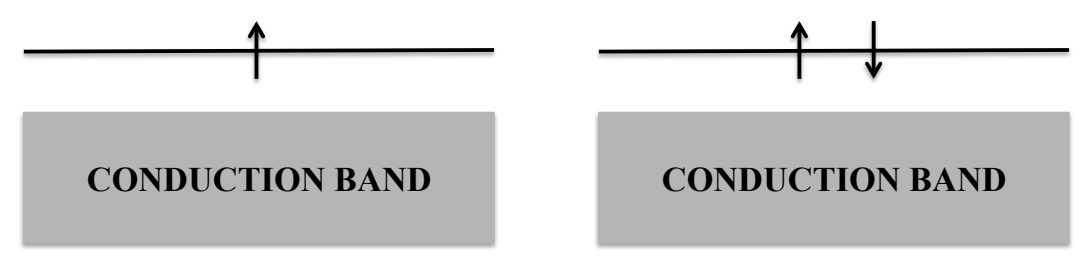

Figure 3.2: Schematic representation of the electronic spectrum for a polyacetylene lattice containing (a) a positive polaron (hole-polaron) and (b) a negative polaron (electron-polaron).

Another type of topological defects can be generated due to the strong electronphonon interaction presented by conjugated polymers. Bipolarons, for instance, which 
are spinless charge carriers possessing charge $\pm 2 e$, may be created in organic semiconductors due to a large concentration of polarons [55,94,98,99,101,102]. For example, two acoustic polarons with the same charge and antiparallel spins can combine with each other to form an acoustic bipolaron. In this way, bipolarons are similar to polarons by presenting similar dimerization pattern and also two localized electronic states inside the band gap. However, regarding the energy levels profile, a bipolaron can be identified by a two states deeper inside the gap when compared to those of a polaron, as shown in Figure 3.3.

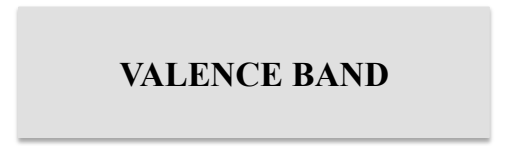

(a)

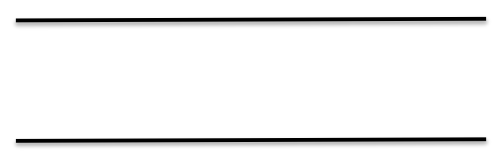

CONDUCTION BAND
VALENCE BAND

(b)

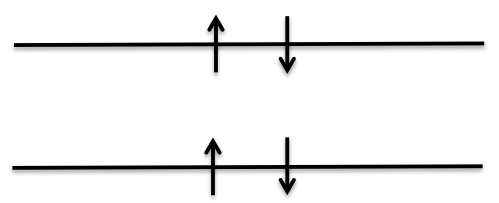

CONDUCTION BAND

Figure 3.3: Schematic representation of the electronic spectrum for a polyacetylene lattice containing (a) a positive bipolaron (hole-bipolaron) and (b) a negative bipolaron (electron-bipolaron).

In contrast to the conventional inorganic conductors, the fact that conjugated polymer are quasi-one-dimensional materials leads to the property of its lattice structure being easily distorted to form self-trapped elementary excitations. Another type of self-localized electronic state are the excitons [35]. In conjugated polymers, an exciton is a bonded state of an electron-hole pair formed due to the strong electron-lattice interactions. As the charge carriers in organic conductors are mainly quasiparticles, the most typical exciton in these material is the one composed of the bound state between a hole (positively charged) polaron and an electron (negatively charged) polaron. Moreover, in these materials the excitons are generally considered to be more strongly localized than excitons in three-dimensional semiconductors, especially because in the former the exciton is substantially confined to a single polymer chain [35].

The spin wavefunction of the exciton, formed from the two spin- $1 / 2$ electronic charges, can be either singlet $(S=0)$ or triplet $(S=1)$. The radiative emission 
(fluorescence) is from the singlet only. The triplet exciton, in its turn, do not produce light emission other than by indirect processes such as phosphorescence or by triplettriplet annihilation [13]. The energy levels configuration for system with an exciton present two localized electronic states inside the band gap, similarly to an organic semiconductor lattice which contains a bipolaron structure, as shown in Figure 3.4.

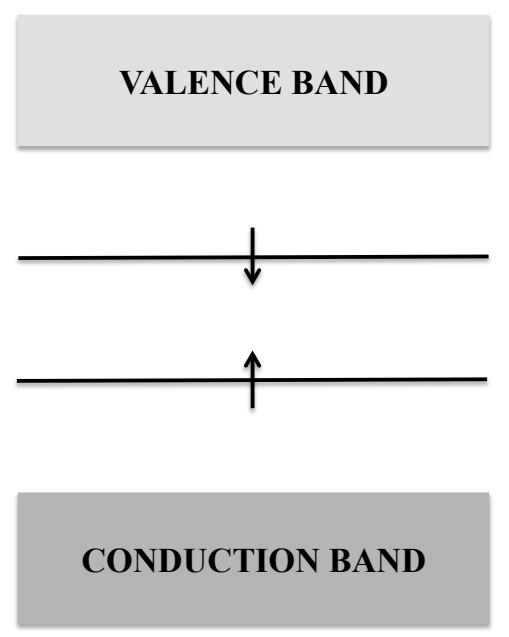

Figure 3.4: Schematic representation of the electronic spectrum for a polyacetylene lattice containing an exciton.

A key aspect in the physics of organic compared to inorganic semiconductors is the difference on the nature of the optically excited states. Whereas in inorganic materials, the production of free charge is carried out directly, the absorption of a photon in organic materials causes a delocalization of these states, which leads to the formation of an exciton due to the strong electron-lattice interactions, as mentioned before $[35,88]$. The organic exciton binding energy is naturally large, on the order of or larger than $500 \mathrm{meV}$. This binding energy represent twenty times or more the thermal energy at room temperature, $k_{B} T(300 \mathrm{~K})=26 \mathrm{meV}$, compared with a few $\mathrm{meV}$ in the case of inorganic semiconductors [35].

Here, is worth to mention some important aspects regarding the most common product found in our studies (a charged excited state), which is generated from the recombination mechanism between charge carriers. As mentioned in the first chapter, a OLED normally consists of a luminescent conjugate polymer layer, introduced between two metal electrodes. Electrons and holes are injected from the electrodes into the polymer layer and, as a result, this process induce self-localized electron states (polarons). It is known that the injected electrons and holes forms electron-polarons and hole-polarons due to the strong electron-lattice interactions in these materials. 
Bipolarons, in their turn, can be created in OLEDs when the charge injection results in a large concentration of polarons. When an electron-polaron (-bipolaron) meets a hole-polaron (-bipolaron), they may collide and recombine to form a mixed state composed by polarons (bipolarons) and excitons (biexcitons), in which the electron and the hole are bonded in a self-trapped lattice deformation known as exciton (biexciton or polaron-exciton (bipolaron-exciton), in analogy to conventional excitons in inorganic semiconductors. The photon emission results from the radiative decay of the charged and neutral excited states. Thus, the yield of these excitations determines the electroluminescence efficiency in conjugated polymers. 


\section{СНАРTER 4}

\section{Conclusions and Perspectives}

The present chapter is intended to serve as an overview about the results presented in the papers which constitute this thesis. Moreover, we discuss an extension of the SSH model performed to analyze the charge transport mechanism in graphene nanoribbons as further perspectives.

In this work, a systematic numerical investigations about the influence of electric field, electron-electron interactions, impurity, and temperature on the recombination mechanism between different kinds of quasi-particles were performed using a polyacetylene chain in the scope of a nonadiabatic evolution method. An Ehrenfest molecular dynamics was performed by using an one-dimensional tight-binding model including lattice relaxation. Combined with the extended Hubbard model (EHM), an extended version of the SSH model was used to include external electric fields, the Brazoviskii-Kirova symmetry breaking term, impurity and temperature effects.

The first two cases analyzed were the intrachain recombination between a mixed state composed by two polarons and one exciton and the interchain recombination of a polaron pair, i. e., two polarons with opposite charges which lie initially in two different chains (see PAPER III). Our findings show that, in the case of two coupled chains (interchain recombination), we observed that polaron recombination does not generate an usual exciton. Rather, this type of recombination leads to an exciton associated with an oscillating dipole between the two chains. Besides, the polaron recombination happens immediately after the coupling between chains is turned on. For single chains (intrachain recombination), our results show that in the absence of temperature, the mixed state composed by two polarons and one exciton remains stable, not generating free carriers. This fact can be attributed to the high level of symmetry that systems, not thermally perturbed, present. When temperature effects are considered, we observed that on a single chain, a polaron-exciton suffers spontaneous dissociation forming a free polaron. These results are in good agreements with experimental data [103] and previous theoretical simulations [49] performed for weakly 
coupled chains bearing a polaron-exciton. We concluded that temperature effects are important in the processes of recombination, particularly in the single-molecule polaron-exciton dissociation process, which does not occur without the influence of temperature. The term "polaron-exciton" is used to denote a quasi-particle which is composed by a mixed state between a polaron and an exciton. It was also observed that the increase of temperature does not influence the time interval between creation and decay of charge carriers. This suggests that the efficiency of organic semiconductors is independent of temperature, which is also in agreement with experimental data [35].

An important recombination mechanism which may contribute to the understanding of electroluminescence process in PLEDs is the intrachain interaction between a polaron pair. In our studies, the interaction intrachain interaction of an electronpolaron (negative structure) and a hole-polaron (positive structure) was investigated, mainly, under the influence of impurities (PAPER V), temperature (PAPER IX), and concentration effects (PAPER XII). The aim of these studies was to investigate the formation of neutral excitations or polaron-exciton states, and their respective yields, from the recombination dynamics between an oppositely charged polaron pair. When the impurity effects are taken into account in the recombination mechanism of an oppositely charged polaron pair, the critical electric field to polaron-exciton formation is lower than in the absence of these effects. Also, the results indicated that the presence of impurities in a conjugated polymer chain, for all electric fields regimes, improves the excitation yield and facilitates the polaron-exciton formation in electric field regimes smaller than $0.7 \mathrm{mV} / \AA$.

Regarding the temperature influence, the results show that there are two channels resulting from the recombination between the polaron pair: channel 1, where a critical temperature regime exists, below which neutral excitation directly forms channel 2, where a dimerized lattice is the resulting product of the collisional process for temperatures higher than the critical value. Furthermore, it is found that both channels depend sensitively on the strength of the applied electric field.

Interesting results arose when the recombination dynamics of more than one polaron pair was investigated. Considering a polymer chain with concentration of polarons varying between 2 and 10 polarons, we were able to determine different regimes in which polaron-excitons or neutral excitations were mainly formed from the collisional processes. These regimes were observed to be dependent on both the electric field strength and on the concentration of the initial charge carriers. Moreover, it was found that, the higher the concentration of charge carriers the more probable it 
is to the recombination process to yield polaron-excitons rather than excitons. Furthermore, higher values of electric field also favor the formation of polaron-excitons until the critical value of $1.0 \mathrm{mV} / \AA$, above which a dimerized chain is achieved. As actual electronic devices present high concentration of charge carriers, this study is of fundamental importance to the understanding of the particular conditions in which the desired kind of quasi-particle is to be formed.

As discussed before, a PLED normally consists of a luminescent conjugate polymer layer, introduced between two metal electrodes. Electrons and holes are injected from the electrodes into the polymer layer and, as a result, this process induce self-localized electron states called polarons. A polaron has spin $\pm 1 / 2$ and a charge $\pm e$. It is know that the injected electrons and holes forms electron-polarons and hole-polarons due to the strong electron-lattice interactions in these materials. Bipolarons, that are spinless charge carriers and possess charge $\pm 2 e$, can be created in PLEDs when the charge injection results in a large concentration of polarons. For example, two acoustic polarons with the same charge and antiparallel spins can combine with each other to for an acoustic bipolaron.

When an electron-bipolaron (negative structure) meets a hole-bipolaron (positive structure), they may collide and recombine to form a mixed state composed by bipolarons and excitons, in which the electron and the hole are bonded in a self-trapped lattice deformation known as biexciton or bipolaron-exciton, in analogy to conventional excitons in inorganic semiconductors. The photon emission results from the radiative decay of the excitations. The yield of these excitations determines the electroluminescence efficiency in conjugated polymers. In this way, a systematic study about the biexciton or bipolaron-exciton formation from the recombination dynamics between oppositely charged bipolarons is of major interest.

In our studies, the intrachain interaction of an electron-bipolaron and a holebipolaron was investigated under impurity and Coulombian interactions; and temperature effects, in PAPER VI and PAPER X, respectively. It is found that the electron-electron interactions play an important role on the yield of excitations after the scattering between the charge carriers in both configurations. Our results show that the charge coupled to the lattice deformation after the scattering is sensitive to on-site an nearest-neighbor Coulomb interactions in the sense that, for higher values, less charge remains coupled to the mixed state composed by bipolarons and excitons. When impurity effects are taken into account, the maximum critical electric field for the formation of states composed of bipolarons and excitons increases compared to a system without impurity effects. Also, the results indicate that the presence of 
impurities in a conjugated polymer lattice, for all electric fields regimes, improves the excitation yield and favors the bipolaron-exciton formation. When temperature effects are taken into account, there are two different channels resulting from the recombination between charged bipolarons. For a given regime, there exists a critical temperature value, below which a biexcitonic state is directly formed. Differently, a dimerized lattice is the resulting product of the collisional process, when the temperature is higher than the critical value. Furthermore, it is found that both channels depend sensitively on the strength of the applied electric field. Obtaining such different regimes and the critical temperature values needed to reach one or the other channel is crucial to fully control the charge carrier density in conductive polymers.

Experiments have shown, through optical and magnetic data, that some doped polymers showed signals involving polarons and bipolarons, which proves the coexistence of these charge carriers in conjugated polymers. Another important excitation in the context of PLEDs is the exciton. Analogously to the conventional charge carriers in these materials, exciton are also structures associated with the strong coupling between charge and phonons and it is linked to a structural deformation. An exciton is created via absorption of a photon but can also be the result of the interaction between two polarons with opposite charges, as discussed before, thus resulting in zero net charge. Since polarons, bipolarons, and excitons coexist in PLEDs, there exists a high possibility of collision between these excitations in order to form new excited states. Studies concerning the interaction between charge carriers and excitons can provide a deeper understanding of the properties of conjugated polymers in general. For the recombination dynamics between a polaron and a bipolaron, the impurity effects and the electron-electron interactions play an important role on the yield of excitations after the interaction between them. In the presence of impurities and for values of on-site Coulomb interactions higher than $0.3 \mathrm{eV}$, less charge remains on the the bipolaron and polaron structures, see PAPER VI. Considering the temperature effects, as discussed in PAPER VIII, the results show that there are two channels resulting from the scattering process between the bipolaron-polaron pair: channel 1 , below the critical temperature regime, where the positive bipolaron and a mixed stated composed of the negative polaron and an exciton are formed, and channel 2, where a positive bipolaron and a free electron are the resulting products of the collisional process, when the temperature is higher than the critical value. Both channels are depend on the strength of the applied electric field.

We have also investigated the intrachain recombination process between two different charge carriers (polaron and bipolaron) and an exciton in conjugated polymers, 
see PAPER XI. The main goal was to find critical values of temperature that induced different products from the collisional process between the quasi-particles. It is shown that, unlike recently proposed at absolute zero temperature, in the case of polarons colliding with excitons, a spin-independent mechanism is obtained when temperature effects are included. This fact demonstrates the importance of considering such effects when describing the recombination process in a more realistic fashion. We performed a thorough description of the polaron exciton collision and determined the critical temperature for obtaining the integrity of the original polaron. We have also found this critical temperature value to be strongly field strength dependent. Regarding the intrachain recombination between a bipolaron and an exciton, two different regimes were also found. For low electric fields and temperatures, the products of the collision were two trions. Otherwise, a final state composed of a polaron and a polaron-exciton is observed.These results on obtaining critical values of controlled parameters for yielding different final states are crucial for mastering the technique of obtaining devices with the desired type and density of charge carriers. Therefore, this knowledge can be useful to improve the performance of organic based electronic devices such as polymer light-emitting diodes.

Although some possible mechanisms for recombination processes between excitons and charge carriers that considered electron-electron interactions and an external electric field have been proposed (see PAPER XI), no detailed theoretical investigations of how two excitons recombine, when these effects are taken into account, have been reported. Furthermore, it has been generally accepted that temperature effects are one of fundamental importance on monomolecular recombination of mixed states composed of polarons and excitons (see PAPER VII).Thus, the exciton recombination in the presence of thermal effects is believed to be essential for OPVs devices. Understanding how the aforementioned effects acts on the monolayer exciton recombination process, which leads to free charge carriers generation, is crucial for the design of more efficient devices and requires a deeper phenomenological description. In this way, the intrachain recombination dynamics of an exciton pair was investigated in our studies, (see PAPER VII). In the simulations the excitons were positioned very close to each other in a way to mimic a high-density region in monomolecular systems. Considering this physical picture, one finds that there are three possible channels resulting from singlet-singlet exciton recombination: (1) formation of an excited negative polaron and an excited positive bipolaron, in the absence of temperature, when the effects over the system of an external electric field and Coulomb interaction are taken into account; (2) generation of two free and excited oppositely charged polarons, when 
thermal effects are considered together with an moderate external electric field regime and electron-electron interactions; and (3) creation of a biexciton in the absence of an external electric field. These results suggest that temperature effects act on the system in a way to decrease the free charge carrier formation when a region with high-density of excitons is considered, whereas electric field effects are of fundamental importance and favors the free charge carrier generation. Moreover, the second channel represents the principal result from the simulations reported in this work, which suggests that there may be an alternative route to creating free charge carriers (hole-polaron and electron-polaron) when a appropriate relation between the density of excitons, electric field, and temperature is considered.

We also turned our attention to an extension of the SSH model performed in our studies to treat the charge transport mechanism in graphene nanoribbons, which may serve as a perspective of forthcoming works.

Graphene-based materials have emerged in the past few years as promising solutions to the design of a novel class of electronic devices. This carbon-based technology is expected to present greater efficiency, lower cost, as well as smaller environmental impact when compared to its inorganic counterpart for photovoltaic and energy storage applications. The strongly covalently bound two-dimensional structure of graphene results in unique properties such as high thermal and electrical conductivities, excellent mechanical strength, and reasonable transparency. Graphene nanoribbons (GNRs) are a special kind of structure derived from specific cuts of a graphene sheet. Besides sharing several of the interesting properties of the two-dimensional original material system, GNRs have, depending on the system symmetry, the possibility of presenting a finite band gap. This feature is of crucial importance for electronics applications. Altogether, this makes GNRs of large interest with regards to both fundamental aspects, such as the basic mechanisms behind charge transport, and more applied studies related to device properties.

It is well-established that low-dimensional carbon-based conductors, in particular, the one-dimensional polymeric system, present the novel property that its lattice structure can be locally distorted to form self-trapped charged states such as polarons. Moreover, it is known from studies of molecular crystals that both electron-lattice interactions and the system dimensionality are of fundamental importance to define the polaron characteristics. The charge associated with the polaron is distributed over the local deformation. It was both theoretically and experimentally reported that the charge carriers in GNRs are formed by more than one nonlocal lattice structure, which 
are symmetric regarding the charge density distribution and the lattice distortion degree, (see PAPER XIII). Interestingly, this structure presents a collective behavior for the charge transport in response of the applied electric field. Because all of these properties are common to regular polarons of other types of carbon-based conductors, we have referred throughout this work to these quasi-particles as polarons.

In one of our studies (see PAPER XIII), the transport of polarons in armchair GNRs was theoretically investigated in the framework of a two-dimensional tightbinding model in which the electron transfer (or hopping) integral includes the electronphonon (e-ph) coupling as well as the force originating from an external electric field. Ehrenfest-like electron-lattice dynamics simulations were carried out in order to investigate the polaron behavior under different conditions of electric fields, GNR widths, and e-ph coupling strengths. Our methodology was able to accurately predict the band gap dependence on the width of the GNRs as well as their values (results are reported in the Supporting Information of PAPER XIII). A careful investigation on the velocity regimes of polarons was also performed. We observed that electric fields favor the occurrence of supersonic polarons. Although the electric field is not able to favor their formation, a role played by e-ph coupling and width, a considerable gain in stability was implemented in the polarons. It was also observed that wider nanoribbons give rise to faster polarons. Their velocities were determined, and an investigation concerning their distribution was performed. Finally, the movement of polarons is followed by different phonons that can, in turn, interact with the polarons, thus affecting their dynamics. 


\section{Bibliography}

[1] Twenty-five years of conducting polymers. Chemical Communications, pages 1-4, 2003, doi: 10.1039/B210718J.

[2] C. P. de Melo. Polímeros condutores. Ciência Hoje, 36:38-42, 1987.

[3] J. A. Epstein and J. S. Miller. Linear-chain conductors. Scientific American, 241:52-61, 1979.

[4] Hideki Shirakawa and S. Ikeda. Preparation and morphology of as-prepared and highly stretch-aligned polyacetylene. Shyntetic Metals, 80:175-184, 1979.

[5] H. Shirakawa. Nobel lecture: The discovery of polyacetylene film - the dawning of an era of conducting polymers. Reviews of Modern Physics, 73:713, 2001.

[6] H. Shirakawa and S. Ikeda. Infrared spectra of poly(acetylene). Polymer Journal, 2:231-244, 1971.

[7] H. Shirakawa, T. Ito, and S. Ikea. Electrical-properties of polyacetylene with various cis-trans compositions. Macromolecular Chemistry and Physics, 179:1565-1573, 1978.

[8] T. Yamabe, K. Akagi, H. Shirakawa, K. Ohzeki, and K. Fukui. Electronicstructure of doped polyacetylene - mechanism of isomerization from cis to trans form. Chemica Scripta, 17:157-158, 1981.

[9] C. K. Chiang, Y. W. Park, A.J. Heeger, H. Shirakawa, E. J. Louis, and A. G. MacDiarmid. Conducting polymers: Halogen doped polyacetylene. Journal of Chemical Physics, 69:5098, 1978.

[10] C.K. Chiang, C.B. Fincher, Y. W. Park, A. J. Heeger, H. Shirakawa, E. J. Louis, S. C. Gau, and A. G. MacDiarmid. Electrical conductivity in doped polyacetylene. Physical Review Letters, 39:1098, 1977.

[11] A. G. MacDiarmid. "shyntetic metals": A novel role for organic polymers. Angewandte Chemie International Edition, 40:2581-2590, 2001. 
[12] J. H. Burroughes, D. D. C. Bradley, A. R. Brown, R. N. Marks, K. Mackay, R. H. Friend, P. L. Burns, and A. B. Holmes. Light-emitting diodes based on conjugated polymers. Nature, 347:539-541, 1990.

[13] R. H. Friend, R. W. Gymer, A. B. Holmes, J. H. Burroughes, R. N. Marks, C. Taliani, D. D. C. Bradley, D. A. Dos Santos, J. L. Brédas, M. Lögdlund, and W. R. Salaneck. Electroluminescence in conjugated polymers. Nature, 397:121-128, 1999.

[14] N. C. Greenham, S. C. Moratti, D. D. C. Bradley, R. H. Friend, and A. B. Holmes. Efficient light-emitting diodes based on polymers with high electron affinities. Nature, 365:628-630, 1993.

[15] G. Li, V. Shrotriya, J. Huang, Y. Yao, T. Moriarty, K. Emery, and Y. Yang. High-efficiency solution processable polymer photovoltaic cells by self-organization of polymer blends. Nature Materials, 4:864-868, 2005.

[16] W. Ma, C. Yang, X. Gong, K. Lee, and A. J. Heeger. Thermally stable, efficient polymer solar cells with nanoscale control of the interpenetrating network morphology. Advanced Functional Meterials, 15:1617-1622, 2005.

[17] H. Sirringhaus, P. J. Brown, R. H. Friend, M. M. Nielsen, K. Bechgaard, B. M. W. Langeveld-Voss, A. J. H. Spiering, R. A. J. Janssen, E. W. Meijer, P. Herwig, and D. M. de Leeuw. Two-dimensional charge transport in self-organized, high-mobility conjugated polymers. Nature, 401:685-688, 1999.

[18] H. Sirringhaus, N. Tessler, and R. H. Friend. Integrated optoelectronic devices based on conjugated polymers. Science, 280:1741-1744, 1998.

[19] H. Sirringhaus, N. Tessler, and R. H. Friend. The path to ubiquitous and low-cost organic electronic appliances on plastic. Nature, 428:911-918, 2004.

[20] R. E: Peierls. Quantum Theory of Solids. Oxford, 1955.

[21] D. J. Burke and D. J. Lipomi. Green chemistry for organic solar cells. Energy E Environmental Science, 6:2053-2066, 2013.

[22] Q. Wang, I. W. H. Oswald, M. R. Perez, H. Jia, A. A. Shahub, Q. Qiao, B. E. Gnade, and M. A. Omary. Doping-free organic light-emitting diodes with very high power efficiency, simple device structure, and superior spectral performance. Advanced Functional Materials, 24:4746-4752, 2014. 
[23] J. H. wan Lee, S. Lee, S. J. Yoo, K. H. Kim, and J. J. Kim. Langevin and trap-assisted recombination in phosphorescent organic light emitting diodes. Advanced Functional Materials, 24:4681-4688, 2014.

[24] R. Shivanna, S. Shoaee, S. Dimitrov, S. Kumar, Kandappa, S. Rajaram, J. R. Durrant, and K. S. Narayan. Charge generation and transport in efficient organic bulk heterojunction solar cells with a perylene acceptor. Energy $E_{6}$ Environmental Science, 7:435-441, 2014.

[25] F. Machui, M. Hosel, N. Li, G. D. Spyropoulos, T. Ameri, R. R. Sondergaard, M. Jorgensen, A. Scheel, D. Gaiser, K. Kreul, D. Lenssen, M. Legros, N. Lemaitre, M. Vilkman, S. Nordman, C. J. Brabeca, and F. C. Krebsb. Cost analysis of roll-to-roll fabricated ito free single and tandem organic solar modules based on data from manufacture. Energy \& Environmental Science, 7:2792$2802,2014$.

[26] A. Bernanose, M. Comte, and P. Vouaux. A new method of emission of light by certain organic compounds. Journal de Chimie Physique, 50:64-68, 1953.

[27] A. Bernanose and P. Vouaux. Organic electroluminescence: Study of transmission mode. Journal de Chimie Physique, 50:261, 1953.

[28] A. Bernanose. The mechanism of organic electroluminescence. Journal de Chimie Physique, 52:396-400, 1955.

[29] A. Bernanose and P. Vouaux. Relationship between the organic electroluminescence device and concentration of active ingredient. Journal de Chimie Physique, 52:509, 1955.

[30] M. Pope, H. K. Kallmann, and P. Magnante. Electroluminescence in organic crystals. Journal Chemical Physics, 38:2042, 1963.

[31] W. Helfrich and W. Schneider. Recombination radiation in anthracene. Physical Review Letters, 14:229-231, 1965.

[32] Y. Lu. Solitons $\&$ Polarons in Conducting Polymers. World Scientifc, 1965.

[33] A. Buckley. Organic light-emitting diodes (OLEDs): materials, devices and applications. Woodhead Publishing, 2013. 
[34] B. P. Rand and H. Richter. Organic Solar Cells: fundamentals, devices, and upscaling. Pan Stanford Publishing, 2014.

[35] B. Kipplen and J. L. Brédas. Organic photovoltaics. Energy E Environmental Science, 2009, pages $=251-261$, volume $=2$.

[36] Z. An, B. Di, and C. Q. Wu. Inelastic scattering of oppositely charged polarons in conjugated polymers. European Physical Journal B, 63:71-77, 2008.

[37] Z. Sun and S. Stafström. Spin-dependent polaron recombination in conjugated polymers. Journal of Chemical Physics, 136:244901-5, 2012.

[38] Zhen Sun and S. Stafström. Bipolaron recombination in conjugated polymers. Journal of Chemical Physics, 135:074902-7, 2011.

[39] Z. Sun, Y. Li, K. Gao, D. S. Liu, Z. An, and S. J. Xie. Dynamical study of polaronâĂŞbipolaron scattering in conjugated polymers. Organic Electronics, 11:279-284, 2010.

[40] Q. Lu, H. Zhao, Y. Chen, and Y. Yan. Dynamics study of the recombination of polaron pairs in polymer chain with impurities. Physica B., 412:13-17, 2013.

[41] J. Lei, Z. Sun, Y. Zhang, and S. Xie. Effect of spin polarization on exciton formation in conjugated polymers. Organic Electronics, 10:1489-1494, 2011.

[42] B. Di, Y. Meng, Y. D. Wang, X. J. Liu, and Z. An. Electroluminescence enhancement in polymer light-emitting diodes through inelastic scattering of oppositely charged bipolarons. Journal of Physical Chemistry B, 115:93399344, 2011.

[43] B. Di, Y. Meng, Y. D. Wang, X. J. Liu, and Z. An. Formation and evolution dynamics of bipolarons in conjugated polymers. Journal of Physical Chemistry B, 115:964-971, 2011.

[44] Yuan Li, Kun Gao, Zhen Sun, Sun Yin, De sheng Liu, and Shi jie Xie. Intrachain polaron motion and geminate combination in donor-acceptor copolymers: Effects of level offset and interfacial coupling. Physical Review B, 78:014304-13, 2008.

[45] Z. Sun, D. Liu, S. Stafstöm, and Z. An. Scattering process between polaron and exciton in conjugated polymers. Journal of Chemical Physics, 134:044906$5,2011$. 
[46] Zhen Sun, Y. Li, D. S. Li, Z. An, and S. Xie. Scattering process between bipolaron and exciton in conjugated polymers. Physical Review B, 134:201310(R), 2009 .

[47] B. Di, Z. An, Y. C. Li, and C. Q. Wu. Effects of e-e interactions on the dynamics of polarons in conjugated polymers. European Physics Letters, 79:17002-5, 2007.

[48] H. Zhao, Y. G. Chen., X. M. Zhang, Z. An, and C. Q. Wu. Correlation effects on the dynamics of bipolarons in nondegenerate conjugated polymers. Journal of Chemical Physics, 130, 2009.

[49] P. H. de Oliveira Neto, W. F. da Cunha, and G. M. e Silva. Charge carrier untrapping by temperature effects in conjugated polymers. European Physics Letters, 88:67006, 2009.

[50] L. F. Roncaratti, R. Gargano, and G. M. e Silva. Theoretical temperature dependence of the charge-carrier mobility in semiconducting polymers. J. Phys. Chem. A., 113:14591-14594, 2009.

[51] Y. H. Yan, Z. An, and C. Q. Wu. Dynamics of polaron in a polymer chain with impurities. European Physical Journal B, 63:71-77, 2004.

[52] K. Harigaya and A. Terai. Dynamics of polaron in a polymer chain with impurities. Physical Review B, 44:7835-7843, 1991.

[53] K. Harigaya, Y. Wada, and K. Fesser. Impurity distribution and electronic states in doped conjugated polymers in the coherent- potential approximation. Physical Review B, 42:11303-11309, 1990.

[54] A. Kadashchuk, V. I. Arkhipov, C. H. Kim, J. Shinar, D. W. Lee, Y. R. Hong, J-II Jin, P. Heremans, and H. Bässler. Localized trions in conjugated polymers. Physical Review B, 76:235205-10, 2007.

[55] Y. Onodera. Polarons, bipolarons, and their interactions in cis-polyacetylene. Physical Review B, 30:775-785, 1984.

[56] M. P. Lima and G. M. e Silva. Polaron stability under collision with different defects in conjugated polymers. International Journal of Quantum Chemistry, 106:2603-2608, 2006. 
[57] V. Gulbinas, D. Hertel, A. Yartsev, and V. Sundström. Charge carrier photogeneration and recombination in ladder-type poly(para-phenylene): Interplay between impurities and external electric field. Physical Review B, 76:235203, 2007.

[58] Y. Meng, B. Di, X. J. Liu, Z. An, and C. Q. Wu. Interchain coupling effects on dynamics of photoexcitations in conjugated polymers. Journal of Chemical Physics, 128:184903-7, 2008.

[59] Y. Meng, X. J. Liu, B. Di, and Z. An. Recombination of polaron and exciton in conjugated polymers. Journal of Chemical Physics, 131, 2009.

[60] D. Moses, A. Dogariu, and A. J. Heeger. Mechanism of carrier generation and recombination in conjugated polymers. Shyntetic Metals, 116, 2001.

[61] C. Soci, D. Moses, and A. J. Heeger. Effects of bimolecular recombination and charge-trapping on the transient photoconductivity of poly(p-phenylene vinylene). Shyntetic Metals, 153, 2005.

[62] C. Soci, D. Moses, Q. H. Xu, and A. J. Heeger. Charge-carrier relaxation dynamics in highly ordered poly(p-phenylene vinylene): Effects of carrier bimolecular recombination and trapping. Physical Review B, 72, 2005.

[63] D. I. Bower. An Introduction to Polymer Physics. Cambridge, 2002.

[64] G. Strobl. The Physics of Polymers. Springer, 1997.

[65] A. Szabo and N. S. Ostlund. Modern Quantum Chemistry. Dover Publications, 1996.

[66] P. Atkins and R. Friedman. Molecular Quantum Mechanics. Oxford, 2005.

[67] J. J. Sakurai. Modern Quantum Mechanics. Pearson, 2014.

[68] J. M. Ziman. Principles of the Theory of Solids. Cambridge, 1972.

[69] A. Einstein. Investigations on the Theory of Brownian Movement. Dover Publications, 1956.

[70] D. S. Lemos. An Introductions to Stochastic Processes in Physics. The Johns Hopkins University Press, 2002. 
[71] G. W. Ford and M. Kac. On the quantum lavegevin equation. Journal of Statistical Physics, 46, 1986.

[72] J. Luczka. Non-markovian stochastic processes: Colored noise. Chaos, 15, 2005.

[73] T. Tomé and M. J. Oliveira. Dinâmica Estocástica e Irreversibilidade. Editora da Univerisade de São Paulo, 2001.

[74] W. P. Su, J. R. Schrieffer, and A. J. Heeger. Solitons in polyacetylene. Physical Review Letters, 42:1698-1701, 1979.

[75] W. P. Su, J. R. Schrieffer, and A. J. Heeger. Soliton excitations in polyacetylene. Physical Review B, 22:2099-2111, 1980.

[76] A. J. Heeger, S. Kivelson, J. R. Schrieffer, and W. P. Su. Soliton in conducting polymers. Reviews of Modern Physics, 60:781, 1988.

[77] J. C. Chiang and A. G. MacDiarmid. Polyaniline - protonic acid doping of the emeraldine form to the metallic regime. Shyntetic Metals, 13:193-205, 1986.

[78] H. Shirakawa, E. J. Louis, A. G. MacDiarmid, C. K. Chiang, and A. J. Heeger. Synthesis of electrically conducting organic polymers - halogen derivatives of polyacetylene chx. Journal of the Chemical Society - Chemical Communications, 16:578-580, 1977.

[79] A. J. Heeger. Semiconducting and metallic polymers : The fourth generation of polymeric materials (nobel lecture). Angewandte Chemie, 40:2591-2611, 2001.

[80] A. J. Heeger. Semiconducting polymers: the third generation. Chemical Society Reviews, 39:2354, 2010.

[81] S. Stafström. Electron localization and the transition from adiabatic to nonadiabatic charge transport in organic conductors. Chem. Soc. Rev., 39:2484-2499, 2010 .

[82] C. S. Pinheiro and G. M e Silva. Use of polarons and bipolarons in logical switches based on conjugated polymers. Phys. Rev. B., 65:094304-5, 2002.

[83] A. Johansson and S. Stafström. Nonadiabatic simulations of polaron dynamics. Phys. Rev. B., 69:235205-7, 2004. 
[84] A. Johansson and S. Stafström. Polaron dynamics in a system of coupled conjugated polymer chains. Phys. Rev. Lett., 86:3602-3605, 2001.

[85] G. M. e Silva and A. Terai. Dynamics of solitons in polyacetylene with interchain coupling. Phys. Rev. B., 47:12568-12577, 1993.

[86] G. M. e Silva. Electric-field effects on the competition between polarons and bipolarons in conjugated polymers. Phys. Rev. B., 61:10777-10781, 2000.

[87] M. P Lima and G. M. e Silva. Dynamical evolution of polaron to bipolaron in conjugated polymers. Phys. Rev. B., 74:224303-6, 2006.

[88] Serguei Brazovskii and Natasha Kirova. Physical theory of excitons in conducting polymers. Chem. Soc. Rev., 39:2453-2465, 2010.

[89] Z. An, C. Q. Wu, and X. Sun. Dynamics of photogenerated polarons in conjugated polymers. Phys. Rev. Lett., 93:216407-4, 2004.

[90] Z. G. Yu, M. W. Wu, X. S. Rao, and A. R. Bishop. Excitons in two coupled conjugated polymer chains. J. Phys. Condes. Matter, 8:8847-8857, 1996.

[91] William Barford. Excitons in conjugated polymers: A tale of two particles. J. Phys. Chem. A., 25:2665-2671, 2013.

[92] William Barford. Theory of singlet exciton yield in light-emitting polymers. Phys. Rev. B., 70:205204-205212, 2004.

[93] J. A. Izaguaire, D. P. Catarello, J. M. Wozniak, and R. D. Skell. Langevin stabilization of molecular dynamics. J. Chem. Phys., 114:2090-2098, 2001.

[94] S. A. Brazoviskii and N. Kirova. Excitons, polarons and bipolarons in conducting polymers. Soviet Physics: Journal of Experimental and Theoretical Physics (JETP), 33:4-8, 1981.

[95] D. S. Brodeaux, R. R. Chance, J. L. Brédas, and R. Silbey. Solitons and polarons in polyacetylene: Self-consistent-filed calculations of the effect neutral and charged defects on molecular geometry. Physical Review B, 28:6927-6936, 1983.

[96] Y. R. Liu and K. Maki. Two-soliton interaction energy and the soliton lattice in polyacetylene. Physical Review B, 22:5754-5758, 1980. 
[97] Y. Onodera and S. Okuno. Two-polaron solution and its stability in the continuum model of polyacetylne. Journal of Physics Society of Japan, 52:2478-2484, 1983.

[98] S. Stafström and K. A. Chao. Polaron-bipolaron - soliton doping in polyacetylene. Physical Review B, 30:2098-2103, 1984.

[99] J. L. Brédas and G. B. Street. Polarons, bipolarons, and solitons in conducting polymers. Accounts of Chemical Research, 18:309-315, 1985.

[100] C. Khun. Solitons, polarons, and excitons in polyacetylene. Physical Review B, 40:7776, 1989.

[101] D. K. Campbell and A. R. Bishop. Solitons in polyacetylene and the relativistic field theory model. Physical Review B, 24:4859-4862, 1981.

[102] C. W. Lang, Z. B. Su, and F. Martino. Bipolaron dynamics in nearly degenerate quasi-one-dimensional polymers. Physical Review B, 33:1512-1515, 1986.

[103] S. H. Kim, T. Zyung, H. Y. Chu, L. M. Do, and D. H. Hwang. Charge transport in a pi-conjugated polymer: Generalized langevin equation analysis. Phys. Rev. B., 61:15854, 2000. 


\title{
appendix $A$ PAPER I
}

\section{Predicting the Equilibrium Structure of Organic Semiconductors with Genetic Algorithms}

\author{
Luiz Antonio Ribeiro Junior \\ Pedro Henrique de Oliveira Neto \\ Wiliam Ferreira da Cunha \\ Ricardo Gargano \\ Geraldo Magela e Silva
}

Chem. Phys. Lett., 555, 168-172 (2013) 


\title{
Predicting the equilibrium structure of organic semiconductors with genetic algorithms
}

\author{
Luiz Antonio Ribeiro Jr., Pedro Henrique de Oliveira Neto, Wiliam Ferreira da Cunha, Ricardo Gargano*, \\ Geraldo Magela e Silva
}

Institute of Physics, University of Brasilia, Brasilia 70.919-970, Brazil

\section{A R T I C L E I N F O}

\section{Article history:}

Received 5 September 2012

In final form 24 October 2012

Available online 1 November 2012

\begin{abstract}
A B S T R A C T
We propose a genetic algorithm optimization technique (GAOT) designed to obtain the lattice geometry parameters and the electronic structure of $\pi$-conjugated polymer systems. Particularly, we obtain the ground state structure of a completely dimerized trans-polyacetylene chain $(\mathrm{CH})_{x}$ using this approach. We also use this approach to determine the polaron and soliton solutions. These solutions are then compared to the traditional self-consistent-field (SCF) solutions. We conclude that the optimization technique proposed here generates solutions as those obtained using traditional (SCF) techniques, with the important advantage of reducing significantly the number of iterations needed to reach convergence. Besides, the convergence of the GAOT technique is more robust, reaching solutions independently of initial guesses.
\end{abstract}

(c) 2012 Elsevier B.V. All rights reserved.

\section{Introduction}

The potential of organic semiconductors in the development of new optoelectronic devices has attracted the interest of the scientific community in recent years [1,2]. Applications of these materials include light emitting diodes (OLEDs) [3,4], thin film transistors $[5,6]$, photodiodes and photovoltaics (OPVs) [7,8]. A significant challenge to make efficient use of these new materials is the accurate prediction of their geometrical and electronic properties. It is known that, in $\pi$-conjugated polymers, the low-energy structure displays a pattern of alternate single and double bonds forming a dimerized polymeric chain with two phases [9]. The determination of this ground state structure is crucial to the analysis of different optical and electronic properties in these materials.

Genetic algorithm optimization techniques (GAOTs) are a class of computational methods based on evolution to quickly determine a potential solution for a specific problem. These algorithms allow the development of generalized methods of search and optimization that attempts to solve by trial and error, complex problems. The underline idea is to simulate the natural processes of evolution without human intervention. Genetic algorithms have been applied successfully in the description of a variety of global minimization and optimization problems [10-17], geometrical structures predictions [18], and fitting of the potential energy surfaces [19-23]. Theoretical studies about ground state structures and low-energy configuration predictions have been extensively performed recently [24-28].

\footnotetext{
* Corresponding author. Fax: +55 6133072363 .

E-mail addresses: gargano@unb.br, gargano@fis.unb.br (R. Gargano).
}

Another interesting feature of genetic algorithms is that they allow mapping of the phase space. This feature allows for the location not only of global minimum but also of all local minima that describes the system. In this sense, Xiang et al. analyzed the structure and the electronic properties of metallic and organic systems using a genetic algorithm to search the global lowest-energy structures [29,30]. In the case of organic systems, a genetic algorithm was used in conjunction with DFT to search for the global minimum structures of oxidized graphene in different oxidation states [29]. Initially, they consider only an arrangement of epoxide groups on single-layer graphene. Using this starting point, they found two new low-energy semiconducting phases of the fully oxidized graphene.

In the present work we developed a program endowed with the ability to self-adapt as it searches for the ground state of both neutral and charged trans-polyacetylene molecules. Specifically, a systematic numerical investigation is performed to find the positions of each monomer describing the minimal energy (equilibrium) configuration within the Su-Schrieffer-Heeger (SSH) model. In these materials, solitons and polarons are the typical structures responsible for charge transport (free charge carriers) [31-33] All the properties of the charge carrier including dynamics [34,35], photogeneration mechanisms [36], processes of excitons dissociation, and charge carriers recombination [36-38] use ground state structures as a starting point. The advantage of our method is that GA convergence is reached in a considerably smaller number of iterations when compared with the usual SCF.

We show that results obtained using GA are equivalent to those obtained using conventional SCF models. To do this comparison we used polaron and soliton type solutions. In some cases, SCF-based 
methods has convergence difficulties because these methods walk through the phase space based on the initial configuration guess. On the other hand, GAOT is designed to search the global minimum of the system regardless of the initial conditions. Due to its robustness, GAOT always converges to the global minimum avoiding the premature convergence to a local minima. These results show that GAOT combined with the SSH model can be successfully applied to find the ground state structure of organic semiconductors reducing the number of iterations needed to reach convergence.

\section{Methodology}

We choose a polyacetylene chain in trans configuration to demonstrate the prediction of the ground state structure of a conjugated polymer chain of finite length, using the methodology developed here. An SSH-type Hamiltonian [39,40] is used to describe the polymer.

$H=-\sum_{n, S} t_{n, n+1}\left(C_{n+1, s}^{\dagger} C_{n, s}+\right.$ h.c $)+\sum_{n} \frac{K}{2} y_{n}^{2}+\sum_{n} \frac{p_{n}^{2}}{2 M}$

Here $n$ indexes the sites. The operator $C_{n+1 s}^{\dagger}$ creates and the operator $C_{n, s}$ annihilates a $\pi$-electron at the $n$-th site with spin $s . K$ is the harmonic constant due to the $\sigma$ bonds. $M$ is the mass of a $\mathrm{CH}$ group. $y_{n} \equiv u_{n+1}-u_{n}$, where $u_{n}$ is the lattice displacement of the atom at the $n$-th site along the chain. $p_{n}$ is the momentum conjugated to $u_{n}$ and $t_{n, n+1}$ is the hopping integral, given by

$t_{n, n+1}=\left(t_{0}-\alpha y_{n}\right)$

with $t_{0}$ being the hopping integral of a $\pi$-electron between nearest neighbor sites in the undimerized chain, and $\alpha$ is the electronlattice coupling constant. The parameters chosen in this work are $t_{0}=2.5 \mathrm{eV}, M=1349.14 \mathrm{eV} \times \mathrm{fs}^{2} / \AA^{2}, K=21 \mathrm{eV \AA} \AA^{-2}$ and $\alpha=$ $4.1 \mathrm{eVA}^{-1}$. These values have been used elsewhere [34-38] with a good track record and the results are expected to be valid for other conjugated polymers. Nevertheless, it should be pointed out that the use of the GAOT in three-dimensional models of organic semiconductors is straightforward. One should just substitutes the SSH by the chosen model and takes $y_{n}$ as the distance between atoms.
The GAOT finds the set of $u_{n}$ 's that provides the lowest energy starting from a randomly generated set of $u_{n}$ 's. The population is composed of $N_{\text {pop }}$ individuals. Each individual has a genetic code with $N$ genes $\left(u_{n}\right)$. These genes are represented by real numbers. The initial population genes are randomly generated. Figure 1 presents a diagrammatic representation explaining how the GAOT works.

Our GAOT uses common genetic algorithm operations: selection, matching with recombination and mutation. The probability of each individual to be selected for recombination takes place by a roulette spinning depending on its fitness [34]. We selected $N_{\text {pop }} / 2$ individuals (parents) that will generate, through the recombination operation, $N_{\text {pop }} / 2$ new individuals (offspring). The new generation, with $N_{\text {pop }} / 2$ parents and $N_{\text {pop }} / 2$ new strings (offsprings) maintains the population with a fixed number $N_{\text {pop }}$.

The original aspect of our genetic algorithm concerns the crossing of genes. Since each gene $\left(u_{n}\right)$ is represented by a real number, whenever two corresponding genes of two parents are chosen to recombine, the offspring genes are determined as follows: each pair of real numbers generates two new numbers following a GAusSIAN probability distribution centered at their mean value and with a dispersion proportional to their difference. This procedure warrants the similarity between the genes of the parents and offsprings, but it also enables the possibility of the offspring genes being higher or lower than their parent values.

The mutation operation is also performed following a GAUSSIAN probability distribution. This time it is centered at a given gene, $u_{n}$. The program selects randomly one gene belonging to a given individual to mutate. The probability of mutation of a given gene equals $0.01 \%$ per generation.

It is quite convenient that the global minimum solution should be found only by a group of individuals in the population. When the entire population converges prematurely to a single solution, this solution may be a local minimum. This is called premature convergence and it can be avoided by the linear scaling. This procedure enhances the probability that several minima will coexists in the population [41]. It introduces a parameter to control the selection pressure, defined as the degree to which the better

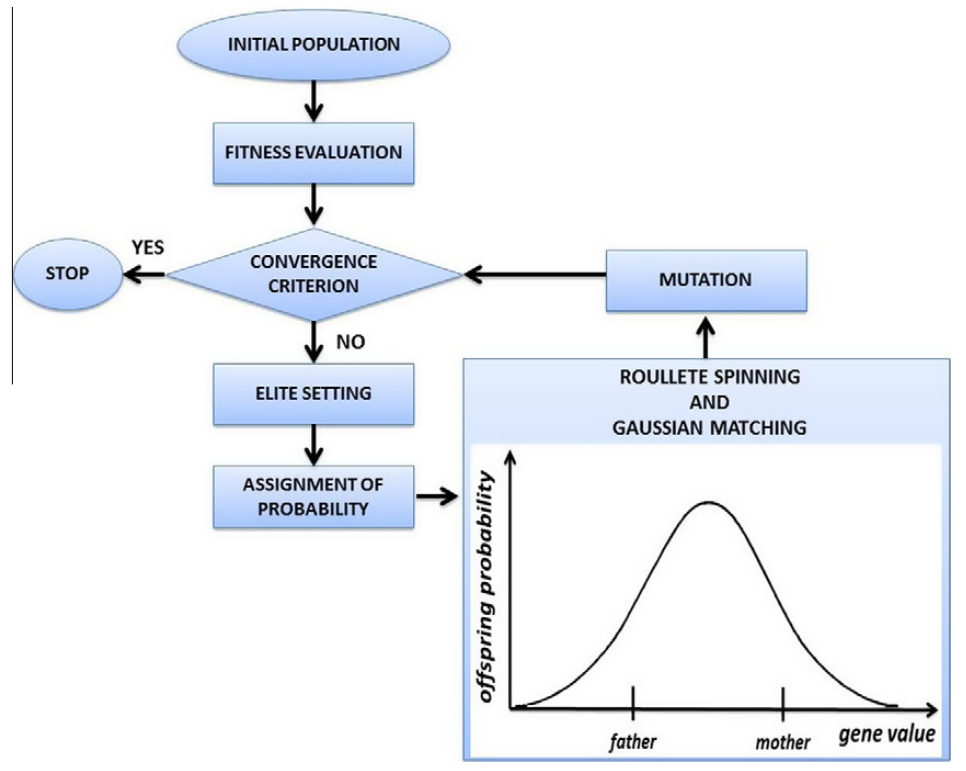

Figure 1. A diagrammatic representation explaining how the GAOT works. 
individuals are favored. Using this approach and varying the mutation rate, we maintain the variety of the population and avoid premature convergence.

Another important procedure implemented in our GAOT is the elitist strategy. This procedure consists in copying an arbitrary number of the best individuals belonging to the population to the next generation. It guarantees that these individuals will not be extinguished. In our GAOT $10 \%$ of the population is represented by the best individuals of the previous generation.

Since we are also interested in excited states, as solitons and polarons, the lowest energy criterion is not very convenient. We found that the mean square difference between the input values $y_{n}$ and the calculated values is a good criterion.

To compare the solutions of GAOT and SCF methods, we use the SCF convergence criterion based in the difference between the bond lengths of successive iterations which is called the Convergence Error.

\section{Results}

In this Letter we present the results obtained for a oligomeric chain of 60 sites. The dimerized configuration generates a bond length variable $y_{n}$ of alternating signs. To provide a good visualization of the results, we defined the order parameter for the charge density $\bar{\rho}(e)$, in units of electron charge $(e)$, and bond length $\bar{y}(\AA)$, as follows:

$$
\begin{aligned}
& \bar{\rho}(e)=1-\frac{\rho_{n-1}+2 \rho_{n}+\rho_{n+1}}{4} \\
& \bar{y}(\AA)=(-1)^{n} \frac{-y_{n-1}+2 y_{n}-y_{n+1}}{4} .
\end{aligned}
$$

Figure 2a shows the convergence for the solution of the best individual for 10 different initial populations randomly generated within the same initial interval. In the beginning (up to the 10th generation) the convergence of the solution is essentially identical for all populations. The crossover and mutation operators then begin to make a difference in the convergence which is characterized by a small separation between these curves. The efficiency of the elitist strategy is easily seen in Figure 1 where we note that the convergence of the error never suffers deterioration in value, i.e., the value of the Convergence Error is always less or equal than the value obtained in the previous generation.

Figure $2 \mathrm{~b}$ zooms-in the region related to the last 10 generations showing the pattern of convergence that is characteristic of the use of the elitist strategy. One can also observe that the curves do not change much in the last five generations. This is due to the fact that all individuals have very close fitness and that the GAOT has found the global minimum of the system, which is in our case the ground state structure.

In Figure 3 we present a comparison between the order parameters for the charge density $\bar{\rho}(e)$ and bond length $\bar{y}(\AA)$ for the polaron and soliton static type solutions obtained with the SCF and GAOT methods. This comparison demonstrates the reach of the GAOT to obtain the ground state configurations of organic semiconductors, since the solutions generated by these methods are almost identical.

An important result obtained here is that the GAOT significantly reduces the number of iterations necessary to reach convergence. To compare the number of iterations needed to reach convergence, we performed simulations with polyacetylene chains containing $20,40,80,160,320,640$ and 1280 sites. Figure 4 shows the comparison between the number of iterations needed to reach a solution when GAOT and SCF methods are used. Our results show that the number of iterations is considerably smaller when the GAOT method is used to find the ground state of organic semiconductors.

It should be pointed out that the use of the GAOT does not necessarily translate in saving of CPU time. Its advantage comes from the enhanced possibility of effectively finding the ground state as well as excited state solutions.

\section{Conclusions}

In this work we studied the application of a genetic algorithm optimization technique to investigate the electronic structure of $\pi$-conjugated polymer systems. Specifically, we obtained the ground state structure of a fully dimerized trans-polyacetylene chain, and the polaron and soliton type solutions using the GAOT and SCF methods for comparison purposes. Our results show that GAOT and SCF generate equivalent solutions. However, unlike the $\mathrm{SCF}$, the GAOT is independent of the initial guess. Also, our results suggests that the GAOT significantly reduces the number of iterations needed to reach convergence. From these results, we can conclude that GAOT is a useful minimization tool to predict the ground state and excited states of organic semiconductors.

It is also noted that crossover and mutation operations play an important role in the solution of the problem. These operations make the GAOT go through the phase space maintaining the variety of the individuals in the population. We can also note that the profile of the population in the GAOT gives important information about the studied systems, specifically when we find the distribution of local minima. Understanding this profile and developing tools and methods to map them, like elitist strategy and linear scaling, is important for employing the GAOT in several classes of
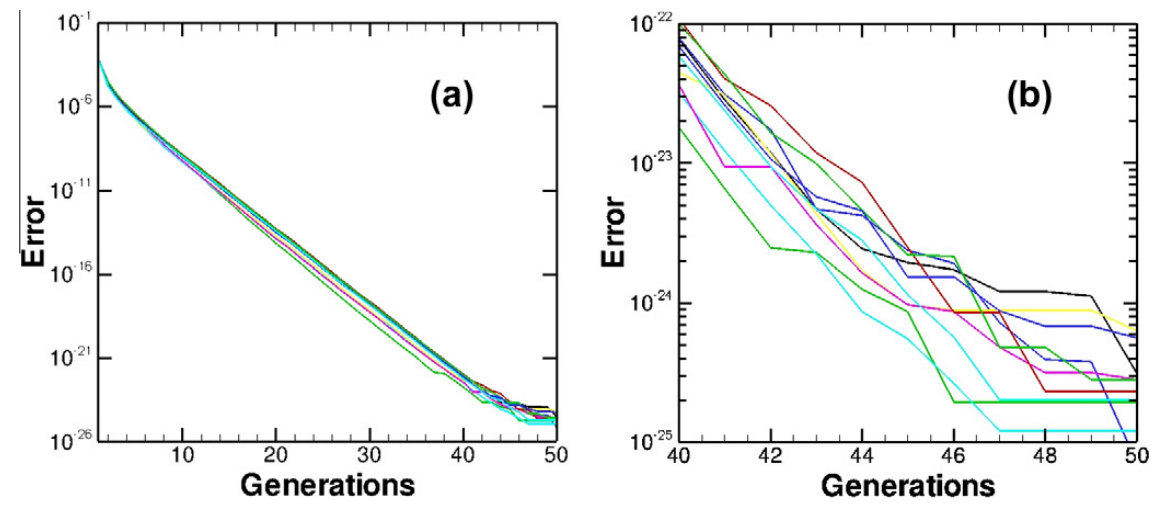

Figure 2. (a) Convergence error for 10 different initial populations. (b) Inset of the convergence for the last 11 generations. 

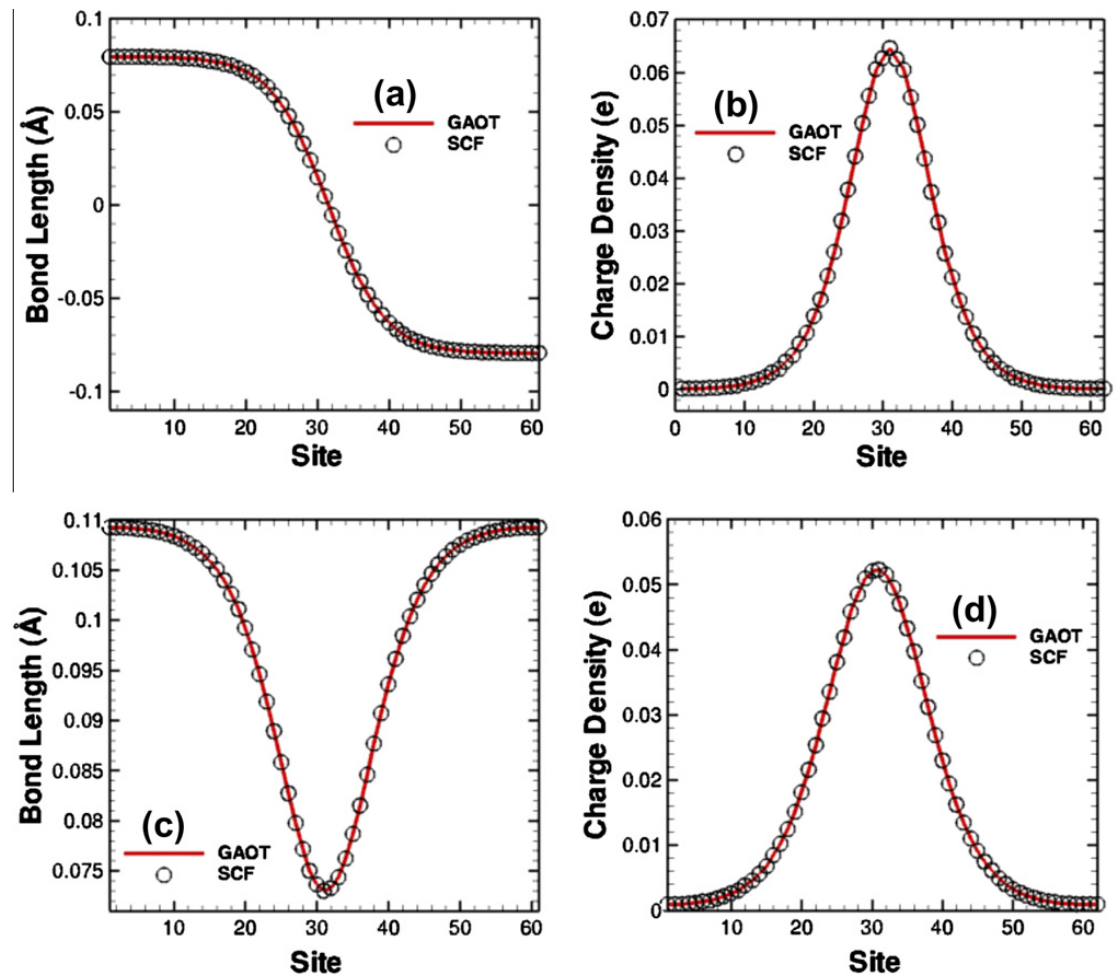

Figure 3. Bond length $\bar{y}(\AA)$ and charge density $\bar{\rho}(e)$ comparison for the soliton (a and b) and polaron (c and d) solutions obtained with the SCF (circle), and GAOT (line) methods.

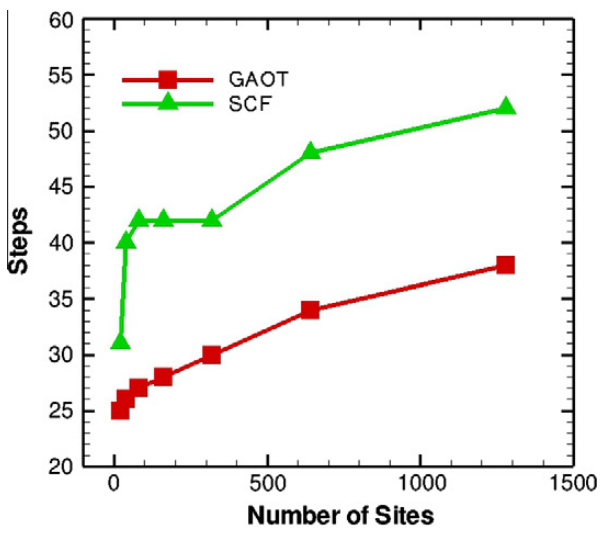

Figure 4. Number of iterations to find the solution versus the chain lengths.

chemical and physical problems. Our numerical results for the ground state and excited states of the organic semiconductors, to the extent that the SSH model could be taken as the standard model for these systems, indicate that this technique could be used to obtain the low-energy configurations in a fast and accurate way.

\section{Acknowledgements}

The authors gratefully acknowledge the financial support from the Brazilian Research Councils CNPq, CAPES and FINATEC.

\section{References}

[1] J.W. Yoo, C.Y. Chen, H.W. Jang, C.W. Bark, V.N. Prigodin, C.B. Eom, A.J. Epstein, Nat. Mater. 9 (2010) 638.

[2] T. Sakanoue, H. Sirringhaus, Nat. Mater. 9 (2010) 737.

[3] S. Reineke, F. Lindner, G. Schwartz, N. Seidler, K. Walzer, B. Lüssem, K. Leo, Nature 459 (2009) 234.

[4] T.D. Nguyen, G. Hukic-Markosian, F. Wang, L. Wojcik, X.G. Li, E. Ehrenfreund, Z.V. Vardeny, Nat. Mater. 9 (2010) 345

[5] B.N. Pal, B.M. Dhar, K.C. See, H.E. Katz, Nat. Mater. 8 (2009) 898

[6] H. Yan et al., Nature 457 (2009) 679

[7] Y. Zhang, G. Hukic-Markosian, D. Mascaro, Z.V. Vardeny, Synth. Metals 160 (2010) 262

[8] S. Singha, Y. Zhang, Z.V. Vardeny, Synth. Metals 160 (2010) 311.

[9] W.P. Su, J.R. Schrieffer, A.J. Heeger, Phys. Rev. Lett. 43 (1979) 1698.

[10] N.L. Abraham, M.IJ. Probert, Phys. Rev. B 77 (2008) 134117.

[11] S. Sharma, H. Singh, G.G. Balint-Kurti, J. Chem. Phys. 132 (2010) 064108.

[12] S. Mallakpour, M. Hatami, H. Golmohammadi, Polymer 51 (2010) 3568.

[13] Q. Feng, Y.Z. Zhang, H.O. Jeschke, Phys. Rev. B 79 (2009) 235112.

[13] Q. Feng, Y.Z. Zhang, H.O. Jeschke, Phys. Rev. B 79

[14] C. Lazo, F.J. Keil, Phys. Rev. B 79 (2000) 245418.

[15] J. Roslund, O.M. Shir, T. Bäck, H. Rabitz, Phys. Rev. A 80

[16] J. Roslund, H. Rabitz, Phys. Rev. A 79 (2009) 053417.
[17] E. Lier, D.H. Werner, C.P. Scarborough, Q. Wu, J.A. Bossard, Nat. Mat. 10 (2011) 7] E. Lier, D.

18] S. Nandy, P. Chaudhury, S.P. Bhattacharyy, J. Chem. Phys. 132 (2010) 234104.

[19] W.M. Brown, A.P. Thompson, P.A. Schultz, J. Chem. Phys. 132 (2010) 024108.

$20]$ J. Xu, H.L. Zhou, Z. Chen, C.D. Lin, Phys. Rev. A 79 (2009) 052508.

[21] L.F. Roncaratti, R. Gargano, G.M. e Silva, Theo. Chem. 769 (2006) 47.

[22] W.F. Cunha, L.F. Roncaratti, R. Gargano, G.M. e Silva, Int. J. Quant. Chem. 106 (2006) 2650.

[23] E. Vitali, M. Rossi, L. Reatto, D.E. Galli, Phys. Rev. B 82 (2010) 174510.

[24] A.L.S. Chua, N.A. Benedek, L. Chen, M. Finnis, A.P. Sutton, Nat. Mat. 9 (2010) 418.

[25] A. Kaur, A.K. Bakhshi, Chem. Phys. 122 (2010) 369.

[26] M. Kahn, J. Weis, C. Kahl J. Chem. Phys. 133 (2010) 224504.

[27] B. Huber, M. Moseler, Phys. Rev. B 80 (2009) 235425.

[28] J. Hooper, A. Ismail, J.B. Giorgi, T.K. Woo, Phys. Rev. B 81 (2010) 224104.

[29] H.J. Xiang, S.H. Wei, X.G. Gong, Phys. Rev. B 82 (2010) 035416.

[30] H.J. Xiang, S.H. Wei, X.G. Gong, J. Am. Chem. Soc. 132 (2010) 7355.

[30] H.J. Xiang, S.H. Wei, X.G. Gong, J. Am. Chem. Soc. 132 (2010) 7355. 
[32] C. Kuhn, Phys. Rev. B 40 (1989) 7776.

[33] G.M. e Silva, Phys. Rev. B 61 (2000) 10777.

[34] P. de Oliveira Neto, W. da Cunha, R. Gargano, G.M. e Silva, J. Phys. Chem. A 113 (2009) 14975

[35] P. de Oliveira Neto, W. da Cunha, G.M. e Silva, Eur. Phys. Lett. 88 (2009) 67006 [36] P. de Oliveira Neto, W. da Cunha, L. Roncaratti, R. Gargano, G. e Silva, Chem. Phys. Lett. 493 (2010) 283
37] Y. Meng, X. Liu, B. Di, Z. An, J. Chem. Phys. 131 (2009) 244502

[38] Y. Meng, B. Di, X. Liu, Z. An, C. Wu, J. Chem. Phys. 128 (2008) 198903.

[39] W.P. Su, J.R. Schrieffer, A.J. Heeger, Phys. Rev. Lett. 43 (1979) 1698.

[40] W. Su, J. Schrieffer, A. Hegger, Phys. Rev. B 22 (1980) 2099

[41] D. Goldberg, Genetic Algorithms in Search, Optimization and Machine Learning, Addison-Wesley, 1989. 


\title{
APPENDIX
APER II
}

Effects of Temperature and Electric Field Induced Phase Transitions on the Dynamics of Polarons and Bipolarons

\author{
Luiz Antonio Ribeiro Junior \\ Wiliam Ferreira da Cunha \\ Pedro Henrique de Oliveira Neto \\ Ricardo Gargano \\ Geraldo Magela e Silva
}

New. J. Chem., 37, 2829-2836 (2013) 


\title{
Effects of temperature and electric field induced phase transitions on the dynamics of polarons
and bipolarons
}

Cite this: NewJ.Chem., 2013 37, 2829

Luiz Antonio Ribeiro, William Ferreira da Cunha, Pedro Henrique de Oliveria Neto, Ricardo Gargano and Geraldo Magela e Silva

\begin{abstract}
The stability of charge carriers in conjugated polymers is investigated in terms of a nonadiabatic evolution method by using an extended version of the Su-Schrieffer-Heeger (SSH) model that includes the effects of an external electric field and temperature. On the basis of this physical picture, different patterns of applied electric field and temperature dependence of polaron and bipolaron kinematics as well as the transitions between different regimes are found. Phase transitions from subsonic to supersonic velocities are also discussed in terms of the system conditions. We were able to describe at which thermal regime each quasi-particle loses its stability and also to determine under which circumstances do the electric field and temperature rise or dampen its motion. The results indicate that thermal effects on polaron and bipolaron stability may provide guidance for improving the charge carrier conduction in organic optoelectronic devices.
\end{abstract}

\section{Introduction}

Conjugated polymers are currently being used as active materials in various organic electronic devices, ranging from disposable radio-frequency tags to organic photovoltaics, ${ }^{1,2}$ thin-film transistors $^{3,4}$ and displays. ${ }^{5,6}$ Organic photovoltaics are particularly attractive due to their potential low cost, light weight, and flexibility of the resulting device. The charge carrier mobility has proven to be directly related to device performance, thus playing an essential role in organic electronics. ${ }^{7,8}$ It is well known that charges added by doping, injection, or photoexcitation will induce self-localized electron states called polarons or bipolarons. ${ }^{9}$ An organic light emitting diode (OLED), for example, normally consists of a luminescent conjugate polymer layer, introduced between two metal electrodes. Electrons and holes are injected from the electrodes into the polymer layer and, as a result, this process induces self-localized electron states called polarons. A polaron has a spin $\pm 1 / 2$ and a charge $\pm e$. It is known that the injected electrons and holes form electronpolarons and hole-polarons due to the strong electron-lattice interactions in these materials. Bipolarons, which are spinless charge carriers and possess charge $\pm 2 e$, can be created in OLEDs when the charge injection results in a large concentration of polarons. For example, two acoustic polarons with the same

Institute of Physics, University of Brasilia, 70.919-970, Brasilia, Brazil charge and antiparallel spins can combine with each other to form an acoustic bipolaron. ${ }^{10}$

A central aspect of the science and technology of these materials is the dynamic behavior of these quasi-particles subjected to certain regimes of electric field and temperature. The understanding of the critical temperature and electric field regimes for the charge carrier stability in these materials, as well as of the transition between these regimes, is crucial for the design of more efficient devices and requires an accurate phenomenological description.

Some relevant theoretical studies carried out by Stafström, ${ }^{11-13}$ e Silva, ${ }^{14,15}$ and Conwell ${ }^{16,17}$ and their coworkers have shown that the external electric field has a significant influence on polaron stability in conjugated polymers. In these works it was noted that, in the presence of strong electric fields, the polaron dissociates due to the fact that the lattice cannot further follow the charge motion. Conwell and coworkers have also investigated the polaron stability and mobility under high electric field regimes from the theoretical point of view. ${ }^{18,19}$ In these works the motion of a polaron in a PPV lattice in the presence of a high electric field was investigated using the SSH model. It was observed that the polaron loses its stability when subjected to an electric field as high as $2 \mathrm{mV} \AA^{-1}$ while moving at supersonic velocities. Using a similar approach, Stafström and coworkers analyzed the polaron dynamics in a system composed of coupled conjugated polymer chains in the 
presence of a high external electric field. ${ }^{13,20}$ Their results show that the polaron becomes totally delocalized for an electric field strength higher than $3 \mathrm{mV} \AA^{-1}$. Typical electric field magnitudes for organic light-emitting diodes (OLEDs) are about $1 \mathrm{mV}^{-1}$; for higher electric fields, such as those applied by Stafström, the excess energy in the system creates too much disturbance in the form of lattice vibrations (phonons) which inhibits the polaron formation. In this context, the critical electric field strength for the charge carrier stability plays an important role in the charge transport efficiency of these materials.

The stability of bipolarons has also been analyzed with respect to collisional processes between these charge carriers with other defects present in a conjugated polymer chain ${ }^{21-25}$ and also when one-site Coulomb interaction was taken into account. ${ }^{26}$ In these studies it was found that there is a regime in which the bipolaron dissociates into a polaron and an excited-polaron. Nevertheless, all the above mentioned discussions take into account the charge carriers dissociation mechanism only in the presence of a high electric field and the collision process with other defects. In the context of a real physical system, in which temperature affects the charge transport efficiency, the process of quasi-particles losing stability is still not fully described. In a real physical system such as an OLED, for instance, the energy excess may, to some extent, be transported away in the form of heat. That might lead to a stabilization of the charge carriers even at field strengths of about $3 \mathrm{mV} \AA^{-1}$. Depending on the temperature, however, a competition between thermal and electric field effects on the stability are of major importance. We expect definite changes in the kinematic behavior of charge carriers before losing their stability. It is the goal of the scientific community of this field to explore these changes in favor of an improved charge transport. In this sense a systematic study of the phase transitions related to the stability of charge carriers is highly desired.

In this work, a systematic numerical investigation of the stability of charge carriers in conjugated polymers is performed in a cispolyacetylene chain in terms of a nonadiabatic evolution method. Polaron and bipolaron stability was investigated in a conjugated polymer chain subjected to different thermal regimes. An Ehrenfest Molecular Dynamics was performed by using a one-dimensional tight-binding model including lattice relaxation; the effects of temperature were included by means of a canonical Langevin equation. Since the goal is to investigate polaron and bipolaron stability, as well as the phase transitions observed in the process of thermal and electric field excitations in cis-polyacetylene chains, an extended version of the SSH model was used to include external electric fields and the Brazovskii-Kirova symmetry breaking term. The aim of this paper is to give a microscopic picture of the loss of polaron and bipolaron stability in conjugated polymers, when the thermal effects are taken into account, and contribute to the understanding of the important processes of charge transport in organic conductors.

\section{Methodology}

A polyacetylene chain in cis configuration ${ }^{28}$ was used to study the charge carrier stability under thermal effects in conjugated polymers. The SSH-type Hamiltonian ${ }^{29}$ modified to include an external electric field and the Brazovskii-Kirova symmetry-breaking term used in this work has the following form:

$$
H=-\sum_{n, s}\left(t_{n, n+1} C_{n+1, s}^{\dagger} C_{n, s}+\text { h.c. }\right)+\sum_{n} \frac{K}{2} y_{n}{ }^{2}+\sum_{n} \frac{p_{n}{ }^{2}}{2 M},
$$

where $n$ indicates the sites of the chain. The operator $C_{n, s}^{\dagger}\left(C_{n, s}\right)$ creates (annihilates) a $\pi$-electron state at the $n$th site with spin $s ; K$ is the harmonic constant that describes a $\sigma$ bond and $M$ is the mass of a $\mathrm{CH}$ group. The parameter $y_{n}$ is defined as $y_{n} \equiv u_{n+1}-u_{n}$ where $u_{n}$ is the lattice displacement of an atom at the $n$th site. $p_{n}$ is the conjugated momentum to $u_{n}$ and $t_{n, n+1}$ is the hopping integral, given by

$$
t_{n, n+1}=\mathrm{e}^{-i \gamma A(t)}\left[\left(1+(-1)^{n} \delta_{0}\right) t_{0}-\alpha y_{n}\right],
$$

where $t_{0}$ is the hopping integral of a $\pi$-electron between nearest neighbor sites in the undimerized chain, $\alpha$ is the electronphonon coupling, and $\delta_{0}$ is the Brazovskii-Kirova symmetrybreaking term, which is used to take the cis symmetry of the polymer into account. $\gamma \equiv e a /(\hbar c)$, with $e$ being the absolute value of the electronic charge, $a$ is the lattice constant, and $c$ is the speed of light. The relation between the time-dependent vector potential $\boldsymbol{A}$ and the uniform electric field $\boldsymbol{E}$ is given by $\boldsymbol{E}=-(1 / c) \boldsymbol{A}(t)$. The parameters used here are $t_{0}=2.5 \mathrm{eV}$, $M=1349.14 \mathrm{eV} \mathrm{fs}^{2} \AA^{-2}, K=21 \mathrm{eV} \AA^{-2}, \delta_{0}=0.05, \alpha=4.1 \mathrm{eV} \AA^{-1}$ and a bare optical phonon energy $\hbar \omega_{Q}=\hbar \sqrt{4 K / M}=0.16 \mathrm{eV}$. These values have been used in previous simulations and are expected to be valid for conjugated polymers in general. ${ }^{30-32}$

In order to solve these equations numerically, first a stationary state that is self-consistent with all degrees of freedom of the system (the lattice and electrons) is obtained. Then, the time evolution of the system is described by the equations of motion. The electronic wave function is the solution of the time-dependent Scrhödinger equation:

$$
i \hbar \dot{\psi}_{k, s}(n, t)=-t_{n, n+1} \psi_{k, s}(n+1, t)-t_{n-1, n}^{*} \psi_{k, s}(n-1, t)
$$

where $k$ is the quantum number that specifies an electronic state. The equation of motion that describes the site displacement and provides the temporal evolution of the lattice is obtained in a classical approach. ${ }^{28,29}$ This equation is written as

$$
\begin{aligned}
F_{n}(t)=M \ddot{u}_{n}= & -K\left[2 u_{n}(t)-u_{n+1}(t)-u_{n-1}(t)\right] \\
& +\alpha\left[B_{n, n+1}-B_{n-1, n}+B_{n+1, n}-B_{n, n-1}\right],
\end{aligned}
$$

where $F_{n}(t)$ represents the force on the $n$th site. Here,

$$
B_{n, n^{\prime}}=\sum_{k, s}^{\prime} \psi_{k, s}^{*}(n, t) \psi_{k, s}\left(n^{\prime}, t\right) C_{M_{1}}
$$

is the term that couples the electronic and lattice solutions. The primed summation represents a sum over the occupied states. By introducing instantaneous eigenstates, the solutions of the time-dependent Scrhödinger equation can be expressed as ${ }^{14}$

$$
\psi_{k, s}\left(n, t_{j+1}\right)=\sum_{l}\left[\sum_{m} \phi_{l, s}^{*}\left(m, t_{j}\right) \psi_{k, s}\left(m, t_{j}\right)\right] \times \mathrm{e}^{\left(-i \varepsilon_{l} \Delta t / \hbar\right)} \phi_{l, s}\left(n, t_{j}\right) .
$$


$\left\{\phi_{l}(n)\right\}$ and $\left\{\varepsilon_{l}\right\}$ are the eigenfunctions and the eigenvalues of the electronic part for the Hamiltonian at a given time $t_{j}$. Eqn (4), which defines the evolution of system, may be numerically integrated as ${ }^{14,27,28}$

$$
\begin{gathered}
u_{n}\left(t_{j+1}\right)=u_{n}\left(t_{j}\right)+\dot{u}_{n}\left(t_{j}\right) \Delta t, \\
\dot{u}_{n}\left(t_{j+1}\right)=\dot{u}_{n}\left(t_{j}\right)+\frac{F_{n}\left(t_{j}\right)}{M} \Delta t .
\end{gathered}
$$

Hence, the electronic wave functions and the lattice displacements at the $(j+1)$ th time step are obtained from the $j$ th time step.

It is possible to make use of the Langevin approach to take thermal effects into account ${ }^{33-35}$ by using a white stochastic signal $\zeta(t)$ as the fluctuation term, i.e., a signal with the following properties: $\langle\zeta(t)\rangle \equiv 0$ and $\left\langle\zeta(t) \zeta\left(t^{\prime}\right)\right\rangle=2 M k_{\mathrm{B}} T \Gamma \delta\left(t-t^{\prime}\right)$. A Stokeslike dissipation term is also included in this formalism. Adding the stochastic and the damping force and rewriting the equation of motion, the following equation is obtained ${ }^{15,30}$
In all cases the dynamics of the system is investigated during 500 fs. The considered polymeric chain has 300 sites with periodic bound conditions, and initially contains a single quasi-particle at the 35 th site in all simulations. To analyze the simulations and provide a good visualization of the results, the following mean charge density $\bar{\rho}(t)$, derived from the charge density $\rho(t)=\sum_{k, s}{ }^{\prime} \psi_{k, s}^{*}(n, t) \psi_{k, s}(n, t)$, and the order parameter $\bar{y}(t)$ were used:

$$
\begin{aligned}
& \bar{\rho}(t)=1-\left[\rho_{n-1}(t)+2 \rho_{n}(t)+\rho_{n+1}(t)\right] / 4, \\
& \bar{y}(t)=(-1)^{n}\left[y_{n-1}(t)-2 y_{n}(t)+y_{n+1}(t)\right] / 4 .
\end{aligned}
$$

The electric field is introduced quasi-adiabatically to avoid undesirable lattice vibrations in the process of accelerating the charge carries through a fast transfer of momentum to the polaron or bipolaron. This was carried out by applying a smooth change of the potential vector strength as:

$$
\boldsymbol{A}(t)= \begin{cases}0 & \text { if } t<0 \\ \left(-c E\left[t_{\mathrm{s}}-\left(\frac{t_{\mathrm{s}}}{\pi}\right) \sin \left(\frac{\pi t}{t_{\mathrm{s}}}\right)\right]\right) / 2 & \text { if } 0 \leq t<t_{\mathrm{off}}, \\ -c\left(t-\frac{t_{\mathrm{s}}}{2}\right) & \text { if } t_{\mathrm{s}} \leq t<t_{\mathrm{off}}, \\ \left(-c E\left[t+t_{\mathrm{off}}-t_{\mathrm{s}}+\left(\frac{t_{\mathrm{s}}}{\pi}\right) \sin \left(\frac{t_{\mathrm{s}}}{\pi}\left(t-t_{\mathrm{off}}+\pi\right)\right)\right]\right) & \text { if } t_{\mathrm{w}} \leq t<t_{\mathrm{off}}+t_{\mathrm{s}} \\ -c E t_{\mathrm{off}} & \text { if }-c E t_{\mathrm{off}}\end{cases}
$$

$$
M \ddot{u}_{n}=-\Gamma \dot{u}_{n}+\zeta_{n}(t)+F_{n}(t) \equiv \tilde{F}_{n}(t) .
$$

The relationship between $\zeta_{n}(t), \Gamma$, and the temperature $T$ of the system is given by the fluctuation dissipation theorem. The damping constant can be determined by low temperature lattice thermal conductivity measurements. The $\Gamma$ value used here has the same order of magnitude as expected from experimental data of Raman spectral line width in polydiacetylene $\left(\Gamma=0.01 \omega_{Q}\right) \cdot{ }^{36}$ The basic idea of the quantum thermal bath (QTB) is to use a Langevin-type approach, where the interatomic forces are calculated quantum mechanically but the nuclei dynamics is described using standard molecular dynamics (MD). A technique proposed by Dammak and collaborators uses a QTB to show that the thermal expansion and heat capacity of a solid can be successfully predicted at low temperatures. ${ }^{37}$

\section{Results and discussion}

In order to study the effect of an external electric field and the thermal bath on the polaron and bipolaron stability, we performed a systematical variation of these properties. For the electric field, the following values were taken into account: $0,0.65,1.3,1.95$, and $2.6 \mathrm{mV} \AA^{-1}$, whereas the temperature values considered were $0,10,20,30,40,50,100,150,200,250$ and $300 \mathrm{~K}$. The temperature of the lattice is obtained through the equipartition theorem by using the kinetic energy of a $\mathrm{CH}$ group, which assumes the form $M\left\langle v_{n}^{2}\right\rangle / 2=k_{\mathrm{B}} T / 2$. with $t_{\mathrm{w}}=10 \mathrm{fs}$ being the width, $t_{\mathrm{s}}=1 \mathrm{fs}$ the electric field turn-on period, and $t_{\text {off }}=500 \mathrm{fs}$ the time of the field being turned off. Thus, after 20 fs the electric field becomes constant in time, in accordance with experiments.

When it comes to charge transport, the drift given to quasiparticles by electric fields play a fundamental role, regardless of the actual conducting polymer application considered, such as organic transistors, organic solar cells or other types of organic photovoltaics. Polarons are defined as non-linear structures that move through the lattice of a conducting polymer polarizing the neighborhood and changing bond lengths at the same time. The investigation of the stability of a polaron is carried out by obtaining the polaron lifetime when it is subjected solely to an electric field. In the simulations performed here it was observed that, for any given temperature, a polaron loses its stability for electric field regimes larger than $3.5 \mathrm{mV} \AA^{-1}$, a fact corroborated by other theoretical results. ${ }^{11,13,17,18}$

It is well known, however, that the application of conducting polymers in optoelectronic devices demands a treatment comprising other features that tend to affect the charge carrier stability in these materials. Among them, the temperature stands out as a property closely related to charge transport both in inorganic and in organic semiconductors, albeit in different senses. Thus, the next natural step is the consideration of both thermal and electric field excitations. Fig. 1 shows the energy levels of the system composed of a polaron at $0 \mathrm{~K}$ (Fig. 2(a)) and $200 \mathrm{~K}$ (Fig. 2(b)) in the presence of an electric field of $2.6 \mathrm{mV} \AA^{-1}$. At $0 \mathrm{~K}$, the presence of typical polaron 


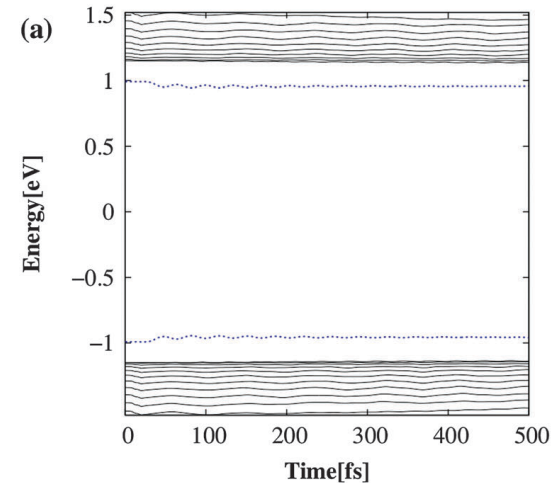

(b)
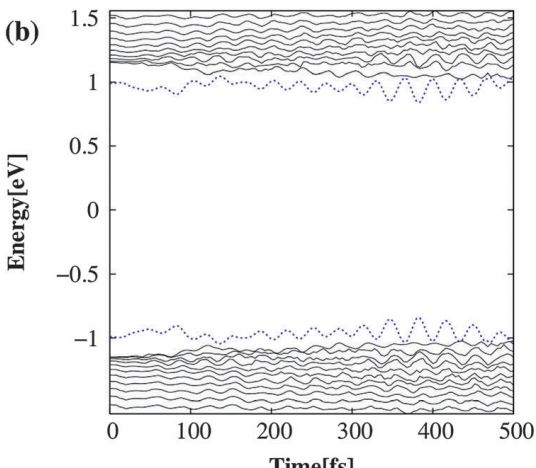

Fig. 1 Time evolution of the energy levels for a polaron subjected to $0 \mathrm{~K}(\mathrm{a})$ and $200 \mathrm{~K}(\mathrm{~b})$

steady states inside the gap can be noted. When considering thermal oscillations, however, we observe the oscillations of the energy levels resulting from the motion of the carbons cores of the lattice, in Fig. 1(b). Initially these oscillations do not give rise to a complete destabilization of the quasi-particle, as can be inferred from the presence of the levels inside the gap. As time evolves, however, the oscillation amplitude increases until the energy levels return to those of conduction and valence bands, a fact that indicates the vanishing of the quasi-particle.

Fig. 2 presents the study performed for the polaron subjected to a constant electric field of $2.6 \mathrm{mV}^{-1}$. The goal is to focus on the effects of temperature on the stability of a moving polaron. In Fig. 2(a) we show the $0 \mathrm{~K}$ simulation of the time evolution of the order parameter in which the smooth trajectory is disturbed only by the accompanying phonons generated by the polaron drift. The linear trajectory presented is directly related to the response of the carrier to the applied field. A completely different pattern is observed in Fig. 2(b), in which thermal effects are taken into account when the temperature regime considered is $150 \mathrm{~K}$. Initially, the polaron moves linearly with time, as in the previous case. However, after about $100 \mathrm{fs}$ the adding up of thermal energy begins to become visible through the blurring of the figure. After about $110 \mathrm{fs}$, the lattice oscillations are of such amplitudes that the polaron can be said
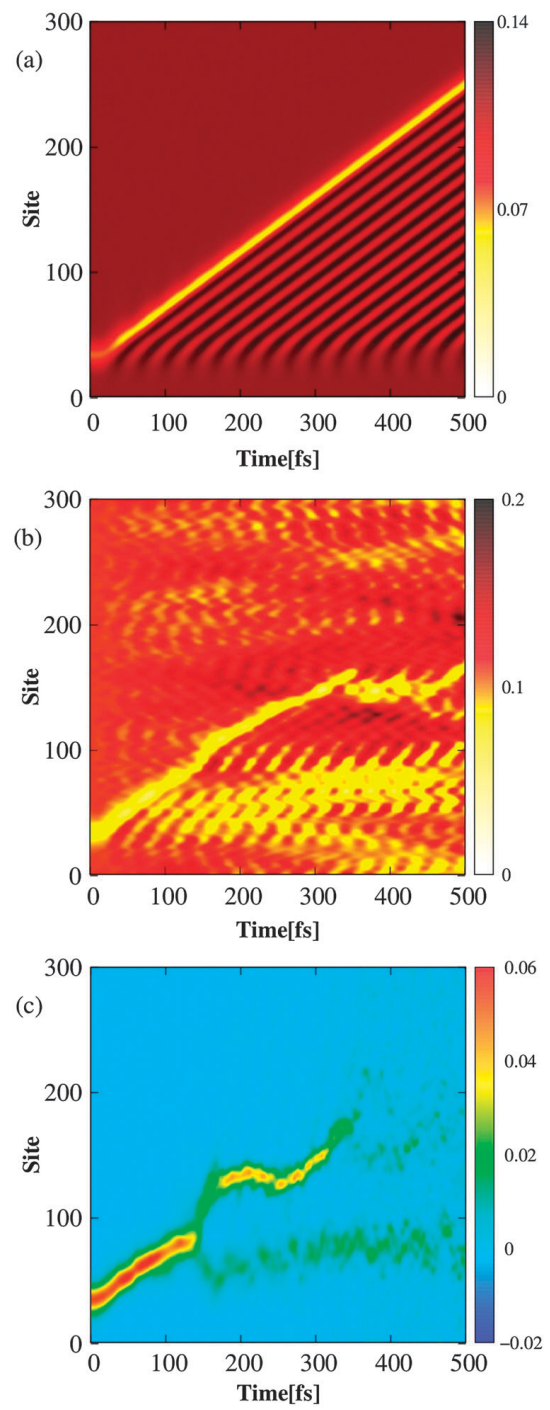

Fig. 2 Time evolution of the order parameter for a polaron subjected to $0 \mathrm{~K}$ (a) and $150 \mathrm{~K}$ (b) and of the charge density of the polaron over $200 \mathrm{~K}$ (c).

to be of reduced stability, although we can still identify such carriers. The disordered pattern of the order parameter shows that the stability reduction of the polaron is indeed caused by the large amplitude of the lattice vibration induced by the temperature. This fact can be confirmed by analyzing Fig. 2(c) which represents the time evolution of the charge density of the system subjected to the temperature regime of $200 \mathrm{~K}$. One can see that once localized charge begins to spread over the lattice until it is completely delocalized at around 350 fs. This delocalization both in geometry and of charge is the typical signature of the absence of the polaron in the system. Thus, Fig. 2(c) presents the annihilation of the polaron when the critical 
(a)

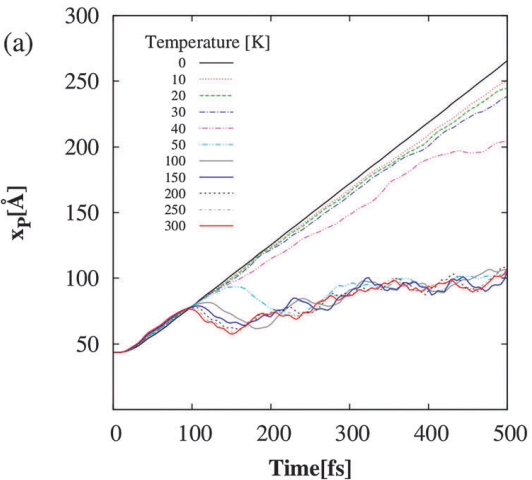

(b)

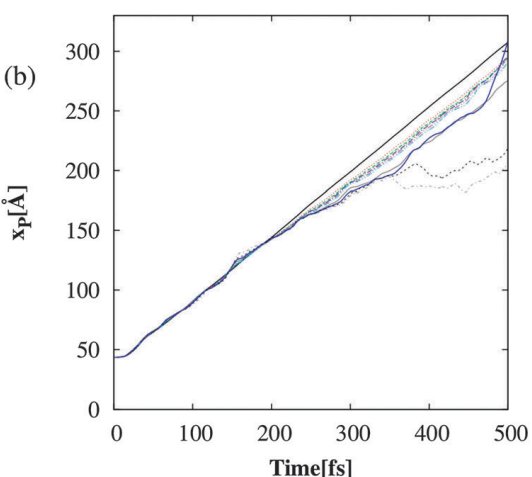

Fig. 3 Trajectory of a polaron subjected to several temperature regimes. (a) Electric field of $1.3 \mathrm{mV} \AA^{-1}$ applied. (b) Electric field of $2.6 \mathrm{mV} \AA^{-1}$ applied.

temperature regime of $200 \mathrm{~K}$ is considered. In summary, a loss of stability of the polaron was observed in this temperature regime, when subjected to an electric field of $2.6 \mathrm{mV} \AA^{-1}$. This result is to be compared to that of a bipolaron later on.

Fig. 3 presents the results for the trajectory of the quasiparticle center of charge on the lattice. The polaron and the bipolaron center positions at a given time $\langle p\rangle(t)$ are calculated from a cyclic mean value of the mean charge density: ${ }^{38}$

$$
\langle p\rangle(t)=\frac{N}{2 \pi} \arg \left\{\left[\sum_{n=1}^{N} \bar{\rho}(t) \mathrm{e}^{i(2 \pi / N) n}\right]\right\} .
$$

The mean velocity $\bar{v}_{p}$ for the polaron and $\bar{v}_{b}$ to the bipolaron is calculated using the center position $\langle p\rangle(t)$ as

$$
\bar{v}=\frac{\langle p\rangle\left(t_{2}\right)-\langle p\rangle\left(t_{1}\right)}{t_{2}-t_{1}}
$$

where for a given time step $t_{1}, t_{2}$ is the next time step.

Each line in Fig. 3(a) corresponds to the simulation of the system at a given temperature subjected to the electric field of $1.3 \mathrm{mV} \AA^{-1}$, whereas in Fig. 3(b) the applied field is of $2.6 \mathrm{mV} \AA^{-1}$. The absence of the $300 \mathrm{~K}$ curve in Fig. 3 (b) is due to the fact that no polaron is stable in this regime. By analyzing the spreading of the curves one can readily note that, the higher the electric field, the less influence the temperature has over the trajectory. An interesting result that takes place in our simulations is the pattern change observed from $40 \mathrm{~K}$ to $50 \mathrm{~K}$. One can see in Fig. 3(a) that for all temperatures lower than $40 \mathrm{~K}$, the trajectories are closer to that of $0 \mathrm{~K}$ when compared to the higher temperatures. The trajectories for temperatures equal to and higher than $50 \mathrm{~K}$ present the same pattern and a much slower mean velocity, which can be measured by the slope of the curves. This fact is attributed to the lattice vibration that disturbs the system to such a level that the electric field is not able to drive a considerable velocity for the already destabilized charge carrier. This consists on a phase transition on the dynamical behavior of the carriers. In the 0 to $40 \mathrm{~K}$ regime, it is possible to note that the higher is the temperature, the greater is the carrier velocity, a property explained by the reasonable delocalization of the charge carrier. At this point it is important to distinguish the delocalization of the polaron, a feature that tends to slightly stretch the quasiparticle over the chain thus increasing its mobility and completely spreading the charge over the system, which as a result, loses the collective behavior that characterizes charge carriers. The former phenomenon is responsible for the positive effect of temperature on charge transport (from $0 \mathrm{~K}$ to $40 \mathrm{~K}$ ) whereas the latter tends to negatively affect this mechanism. This negative influence can be readily noted in Fig. 3(a) through the low velocity exhibited by the quasi-particles of the system subjected to higher temperatures. Fig. 3(b), on the other hand, shows how the application of a higher electric field can be used to stabilize the system. In this case, the system is less sensitive to the effects of temperature due to the fact that the high electric field drives stronger velocities to the carriers. A similar phase transition is observed but this time between 150 and $200 \mathrm{~K}$. In other words, for a higher electric field it takes a greater deal of effort by thermal excitations to destabilize the polaron. For $200 \mathrm{~K}$ and $250 \mathrm{~K}$, a charge carrier annihilation process is observed as the field cannot further implement a collective movement on the charge center after $350 \mathrm{fs}$.

We now turn our attention to bipolarons as charge carriers in organic conductors. In the energy levels profile, a bipolaron can be identified by a couple of states deeper inside the gap when compared to those of a polaron, as can be seen in Fig. 4 for $0 \mathrm{~K}(\mathrm{a})$, and $300 \mathrm{~K}(\mathrm{~b})$. A remarkable result obtained through these simulations is that although the oscillations of the energy levels varied according to the temperature applied, the bipolaron levels were always present, indicating that this particle remained stable despite the high electric field applied and regardless of the temperature considered, as shown in Fig. 4. This result points towards the fact that bipolarons are more stable than polarons. In order to confirm this, a set of simulations analogous to those performed for a polaron was carried out. Fig. 5 presents the time evolution of the order parameter of a bipolaron subjected to an electric field of $2.6 \mathrm{mV} \AA^{-1}$ under the thermal regimes of $0 \mathrm{~K}$ (a) and $150 \mathrm{~K}$ (b) together with the charge density at $200 \mathrm{~K}$ temperature (c). The consistency of the bipolaron is clear in this simulation. Fig. 5(a) represents the smooth trajectory of the bipolaron in the $0 \mathrm{~K}$ regime. 

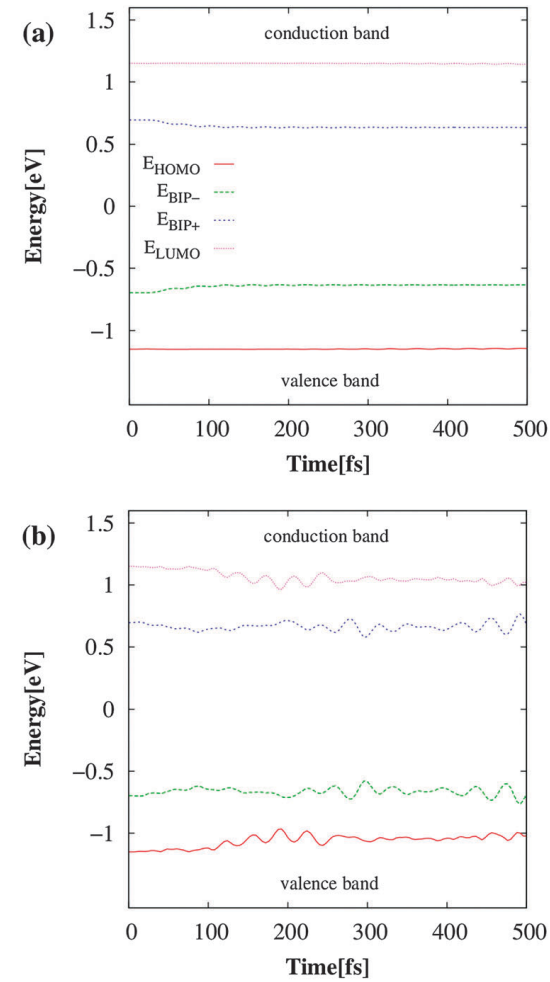

Fig. 4 Time evolution of the energy levels for a bipolaron subjected to $0 \mathrm{~K}$ (a) and $300 \mathrm{~K}(\mathrm{~b})$.

Again, when no thermal excitations are provided, the present phonons are due to the quasi-particle movement and tend to neatly follow the bipolaron. The blurring observed in Fig. 5(b) is a reflection of the presence of thermal phonons that, nevertheless, do not give rise to any bipolaron destabilization, as can be seen by the blue path, which is nothing but the bipolaron distortion, present all through the simulation. Fig. 5(c) presents the time evolution of the charge density order parameter of the system subjected to $200 \mathrm{~K}$. Again, it is possible note the integrity of the quasi-particle until the end of the simulation even for this higher temperature, indicating that, indeed, the bipolaron is fairly stable despite the critical conditions simulated. The trajectory of the bipolarons center of charge is presented in Fig. 6. It is interesting to note that the behavior of this quasi-particle is less sensible to temperature change when compared with the polaron, which can be measured by the closeness between the curves. This feature is closely related to the high stability of bipolarons with respect to the electric field and temperature. As previously discussed, the phase transition has to do with the delocalization of the charge at some point. As for the bipolaron, the charge responding to the applied field is observed for all temperatures and all the fields in Fig. 6(a) and (b), a manifestation of the presence of the charge carrier throughout the whole simulation. Although the
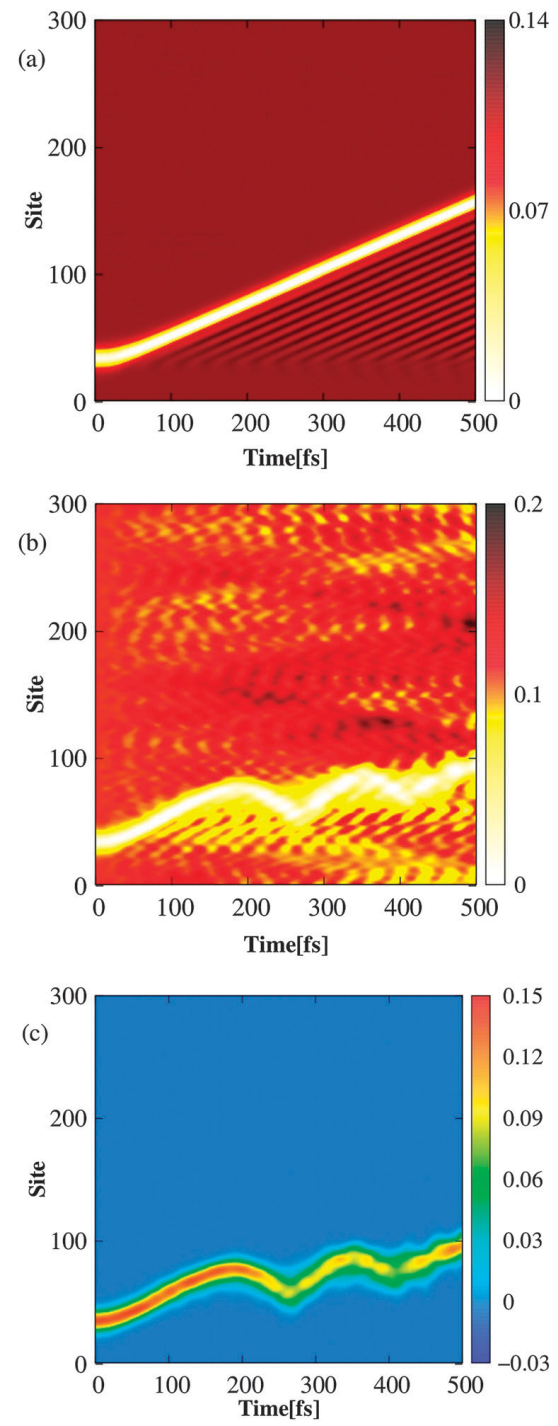

Fig. 5 Time evolution of the order parameter for a bipolaron subjected to $0 \mathrm{~K}$ (a) and $150 \mathrm{~K}$ (b) and of the charge density of the bipolaron under $200 \mathrm{~K}$ (c).

slope of the curves in Fig. 6(a) decreases for temperatures higher than $40 \mathrm{~K}$, it is possible note that all the overall slopes are positive. Naturally as our external conditions of field and temperature increase, as in Fig. 6(b) in which $2.6 \mathrm{mV} \AA^{-1}$ is considered, the pattern tends to look similar to the one of the polaron, particularly for the 250 and $300 \mathrm{~K}$ simulations - the ones that were not even present in the polaron case. Even after this 200-250 K transition, however, an accurate analysis shows that, again, it has positive slopes for all cases. In Fig. 6(b) an even tighter set of curves is observed indicating that the electric field indeed plays the role of stabilizing the system subject to thermal effects. Throughout this work the observed phase 
NJC

$$
\text { (a) }
$$
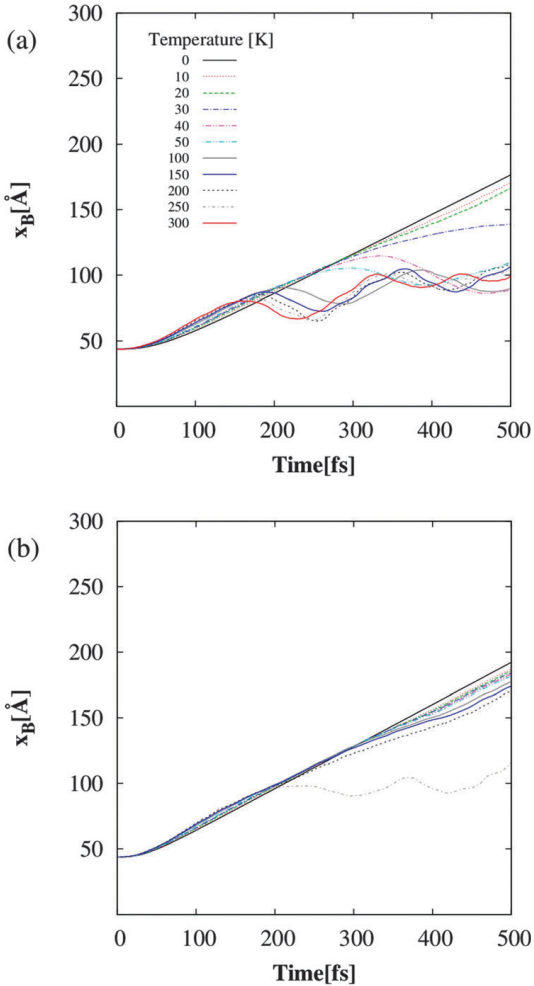

Fig. 6 Trajectory of a bipolaron subjected to several temperature regimes. (a) Electric field of $1.3 \mathrm{mV} \AA^{-1}$ applied. (b) Electric field of $2.6 \mathrm{mV} \AA^{-1}$ applied.

transitions in terms of qualitative slopes of the trajectory plots have been discussed. In Fig. 7, we present a quantitative investigation of the charge carriers velocity. Fig. 7(a) presents the bipolaron velocities plots; Fig. 7(b) is related to polarons and Fig. 7(c) shows a comparison between the two quasi particles. The quasi-particle velocity is plotted as a function of the electric field and each curve represents a given temperature. The sound velocity in the material is represented by the solid black line for comparison purposes, thus allowing to identify transitions between subsonic and supersonic regimes. A direct comparison between Fig. 7(a) and (b) leads us to reiterate the conclusion that polarons are structures more sensitive to temperature variations, and the bipolaron curves exhibit a much more uniform pattern. Also, an important property that can be noted in the figure is the saturation of bipolaron velocity, which is much slower than the polaron's. This fact is also expected due to the larger inertia presented by the former. The most interesting feature of Fig. 7, however, is that it provides the regimes in which the quasi-particle moves in the material at a given velocity. Sound velocity is of particular interest and it is observed that at $1.95 \mathrm{mV} \AA^{-1}$, both polarons and bipolarons move in the polymer chain at approximately the speed of sound. Some relevant theoretical works investigated the velocity phase transitions of polarons in trans-polyacetylene ${ }^{12}$
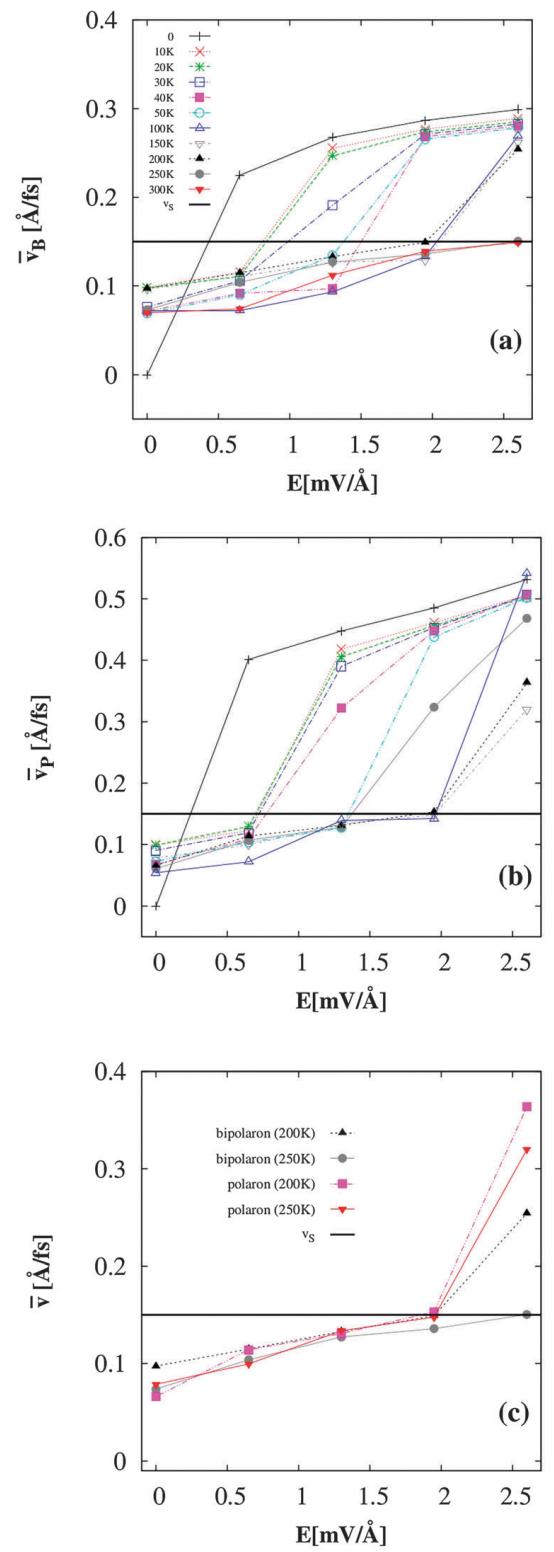

Fig. 7 Velocities of (a) bipolarons, (b) polarons and (c) comparison between them as a function of the applied electric field.

and cis-polyacetylene ${ }^{39}$ chains in the absence of temperature. The results obtained in both works showed a phase transition for the velocity, from the subsonic to the supersonic regime, for electric fields smaller than $0.5 \mathrm{mV}^{-1}$. Also, for this electric field regime, the polaron velocity value reaches $0.35 \AA \mathrm{fs}^{-1}$. In Fig. 7 it is possible to see that the polaron velocity behavior, reported in ref. 12 and 39, changes dramatically when the thermal effects are taken into account. 


\section{Conclusions}

In summary, a modified version of the SSH model was developed to include an external electric field, the Brazovskii-Kirova symmetry breaking term, and temperature effects, in order to investigate the effects of these properties on dynamic phase transitions related to the stability of polarons and bipolarons in cis-symmetry conducting polymers chains. We were able to obtain temperature regimes at which the quasi-particles lose their stability. Furthermore, it was found that bipolarons are more stable than polarons for all regimes of electric field and temperature, remaining stable even at $350 \mathrm{~K}$ and when subjected to an electric field of $2.6 \mathrm{mV} \AA^{-1}$. Depending on the regime considered, the electric field plays the role of stabilizing or destabilizing the quasi-particles. We traced different patterns of electric field and temperature dependence of polaron and bipolaron kinematics, by observing their transitions. Finally, it was possible to predict the velocities of the quasiparticle through the lattice as a function of temperature and electric field and to observe that both polarons and bipolarons move at approximately the speed of sound when subjected to $200 \mathrm{~K}$ or $250 \mathrm{~K}$ at an electric field of $1.95 \mathrm{mV} \AA^{-1}$. The discussion performed here on the behavior of charge carriers and the transitions from one regime to another should help to shed light on the important and exciting field of organic conduction, by providing guidance for the improvement of the charge carrier mobility in organic optoelectronic devices.

\section{Acknowledgements}

The authors gratefully acknowledge the financial support from the Brazilian Research Councils CNPq, CAPES and FINATEC.

\section{References}

1 Y. Sun, G. C. Welch, W. L. Leong, C. J. Takacs, G. C. Bazan and A. J. Heeger, Nat. Mater., 2012, 11, 44.

2 M. T. Greiner, M. G. Helander, W. M. Tang, Z. B. Wang, J. Qiu and Z. H. Lu, Nat. Mater., 2012, 11, 76.

3 D. Braga, N. C. Erickson, M. J. Renn, R. J. Holmes and C. D. Frisbie, Adv. Funct. Mater., 2012, 22, 1623.

4 Y. Qin, D. H. Turkenburg, I. Barbu, W. T. T. Smaal, K. Myny, W. Y. Lin, G. H. Gelinck, P. Heremans, J. Liu and E. R. Meinders, Adv. Funct. Mater., 2012, 22, 1209.

5 A. Perumal, M. Fröbel, S. Gorantla, T. Gemming, B. Lüssem, J. Eckert and K. Leo, Adv. Funct. Mater., 2012, 22, 210.

6 Y. Jin, J. Feng, X. L. Zhang, Y. G. Bi, Y. Bai, L. Chen, T. Lan, Y. F. Liu, Q. D. Chen and H. B. Sun, Adv. Mater., 2012, 24, 1187.

7 V. Coropceanu, J. Cornil, D. A. da Silva Filho, Y. Olivier, R. Silbey and J. L. Brédas, Chem. Rev., 2007, 107, 926.

8 S. Stafström, Chem. Soc. Rev., 2010, 39, 2484.

9 Z. An, C. Wu and X. Sun, Phys. Rev. Lett., 2004, 93, 19.

10 B. Di, Y. Meng, Y. D. Wang, X. J. Liu and Z. An, J. Phys. Chem. B, 2011, 115, 964.
11 Å. Johansson and S. Stafström, Phys. Rev. B: Condens. Matter Mater. Phys., 2002, 65, 045207.

12 Å. Johansson and S. Stafström, Phys. Rev. B: Condens. Matter Mater. Phys., 2004, 69, 235205.

13 Å. Johansson and S. Stafström, Phys. Rev. Lett., 2001, 86, 3602 .

14 G. M. e Silva, Phys. Rev. B: Condens. Matter Mater. Phys., 2000, 61, 10777.

15 L. F. Roncaratti, R. Gargano and G. M. e Silva, J. Phys. Chem. A, 2009, 113, 14591.

16 H. M. Mizes and E. M. Conwell, Synth. Met., 1995, 68, 145.

17 H. M. Mizes and E. M. Conwell, Phys. Rev. Lett., 1993, 70, 1505.

18 D. M. Basko and E. M. Conwell, Phys. Rev. Lett., 2002 , 88, 056401.

19 S. V. Rakhmanova and E. M. Conwell, Appl. Phys. Lett., 1999 , 75, 1518.

20 Å. Johansson and S. Stafström, Synth. Met., 2001, 119, 235.

21 Z. Sun and S. Stafström, J. Chem. Phys., 2011, 135, 074902.

22 Z. Sun, Y. Li, D. S. Liu, Z. An and S. Xie, Org. Electron., 2010, 11, 279.

23 B. Di, Y. Meng, Y. D. Wang, X. J. Liu and Z. An, J. Phys. Chem. $B, 2011,115,9339$.

24 Z. Sun, Y. Li, D. S. Li, Z. An and S. Xie, Phys. Rev. B: Condens. Matter Mater. Phys., 2009, 79, 201310(R).

25 B. Di, Y. Meng, Y. D. Wang, X. J. Liu and Z. An, J. Phys. Chem. $B, 2011,115,964$.

26 H. Zhao, Y. G. Chen, X. M. Zhang, Z. An and C. O. Wu, J. Chem. Phys., 2009, 130, 234908.

27 Y. Ono and A. Terai, J. Phys. Soc. Jpn., 1990, 59, 2893.

28 A. J. Hegger, Angew. Chem., Int. Ed., 2001, 40, 2591.

29 A. J. Hegger, Rev. Mod. Phys., 2001, 73, 681.

30 L. A. Ribeiro, P. H. de Oliveira Neto, W. F. da, L. F. Roncaratti, R. Gargano, D. A. da Silva Filho and G. M. e Silva, J. Chem. Phys., 2011, 135, 224901.

31 L. A. Ribeiro, P. H. de Oliveira Neto, W. F. da Cunha and G. M. e Silva, Phys. Procedia, 2012, 28, 112.

32 L. A. Ribeiro, P. H. de Oliveira Neto, W. F. da Cunha, R. Gargano and G. M. e Silva, Chem. Phys. Lett., 2013, 555, 168.

33 G. W. Ford and M. Kac, J. Stat. Phys, 1987, 46, 803.

34 D. Roy, Phys. Rev. E: Stat. Phys., Plasmas, Fluids, Relat. Interdiscip. Top., 2008, 77, 062102.

35 D. Banerjee, B. C. Bag, S. K. Banika and D. S. Rayb, J. Chem. Phys., 2004, 120, 8960.

36 G. Cerullo, G. Lanzani, M. Z. S. S. de Silvestri, S. D. Comoretto, I. Moggio and G. Dellepiane, Synth. Met., 2001, 116, 57.

37 H. Dammak, Y. Chalopin, M. Laroche, M. Hayoun and J. J. Greffet, Phys. Rev. Lett., 2009, 103, 190601.

38 M. P. Lima and G. M. e Silva, Int. J. Quantum Chem., 2006, 106, 2597.

39 M. V. A. da Silva, P. H. de Oliveira Neto, W. F. da Cunha, R. Gargano and G. M. e Silva, Chem. Phys. Lett., 2012, 550, 146. 


\section{PAPER III}

\section{Exciton Dissociation and Charge Carrier Recombination Processes in Organic Semiconductors}

Luiz Antonio Ribeiro Junior

Pedro Henrique de Oliveira Neto

Wiliam Ferreira da Cunha

Luiz Fernando Roncaratti

Demétrio Antonio da Silva Filho

Ricardo Gargano

Geraldo Magela e Silva

J. Chem. Phys., 135, 224901-6 (2011) 


\title{
Exciton dissociation and charge carrier recombination processes in organic semiconductors
}

\author{
Luiz A. Ribeiro, Pedro H. Oliveira Neto, Wiliam F. da Cunha, Luiz F. Roncaratti, \\ Ricardo Gargano, Demétrio A. da Silva Filho, ${ }^{\text {a) }}$ and Geraldo M. e Silva \\ Institute of Physics, University of Brasilia, Brasilia, 70.919-970, Brazil
}

(Received 13 July 2011; accepted 10 November 2011; published online 9 December 2011)

\begin{abstract}
Exciton dissociation and charge recombination processes in organic semiconductors, with thermal effects taken into account, are described in this paper. Here, we analyzed the mechanisms of polaronexcitons dissociation into free charge carriers and the consequent recombination of those carriers under thermal effects on two parallel $\pi$-conjugated polymers chains electronically coupled. Our results suggest that exciton dissociation in a single molecule give rise to localized, polaron-like charge carrier. Besides, we concluded that in the case of interchain processes, the bimolecular polaron recombination does not lead to an usual exciton state. Rather, this type of recombination leads to an oscillating dipole between the two chains. The recombination time obtained here for these processes are in agreement with the experimental results. Finally, our results show that temperature effects are essential to the relaxation process leading to polaron formation in a single chain, as in the absence of temperature, this process was not observed. In the case of two chains, we conclude that temperature effects also help the bimolecular recombination process, as observed experimentally. (๑) 2011 American Institute of Physics. [doi:10.1063/1.3665392]
\end{abstract}

\section{INTRODUCTION}

The potential of organic semiconductors in the development of new technologies for optoelectronic devices has attracted the interest of both academia and industry in recent years. ${ }^{1-6}$ Among the applications of these materials are light emitting diodes (OLEDs), ${ }^{7-11}$ thin film transistors (OFETs), ${ }^{12-14}$ photodiodes and photovoltaics (OPVs)..$^{15-19}$ Organic photovoltaics are particularly attractive due to its potential low cost, light weight, and flexibility of the resulting device. However, when comparing with conventional solar cells, the underlying science of an organic solar cells is less known. The exciton binding energy in OPVs is $\sim 500 \mathrm{meV}^{20}$ This makes the process of exciton dissociation into free charge carriers much slower, since the thermal energy usually involved in this type of system is $\sim 20 \mathrm{meV}$. Besides, almost all charge carriers in organic semiconductors are generated and suffers recombination on the femtosecond time scale. ${ }^{21}$ The understanding of the generation and decay processes of charge carriers in these materials is crucial for the design of more efficient devices and requires a better phenomenological description.

The dynamics of generation and decay of charge carriers in polymer films of poly-para-phenylene (m-LPPP) by means of femtosecond electromodulated transient absorption spectroscopy were examined in Ref. 22. Using this method, two modes of polaron formation from relaxed exited states (excitons) with different behaviors were identified: (i) impurity induced in the absence of an external electric field and (ii) electric field induced in a pristine film. It was also observed

\footnotetext{
a) Author to whom correspondence should be addressed. Electronic mail: dasf@unb.br.
}

that only a small part of excitons generates charge carriers at zero applied field. The main portion of polarons recombines faster, but a fraction survives up to microseconds and form fluorescent excited states. This fact shows the difficulty in keeping free charge carriers stable enough to generate useful electrical current. Moses et al. analyzed the charge carrier generation and relaxation dynamics using transient excitedstate absorption (photo-induced absorption) measurements in a prototypical luminescent polymer, the poly(phenylene vinylene), probed with 100 fs temporal resolution in the 6-10 mm spectral region. They spanned the infrared active vibrational modes to propose the direct polaron creation, and also to indicate that the charge carrier dissociation rate depends on the strength of the interchain interaction. ${ }^{23-25}$ This result suggests that direct polaron creation can be an alternative route to the generation of stable free charge carriers.

Theoretical studies of the effects of interchain interaction on the recombination processes of charge carrier and on the generation dynamics in polymer chains ${ }^{26-28}$ have been extensively carried out recently. In these studies, a nonadiabatic evolution method was used to investigate the relaxation dynamics of the charge carriers in the presence of an external electric field. The inclusion of electron repulsion terms has provided very interesting results concerning excitons with a Su-Schrieffer-Heeger (SSH) type model for parallel chains. ${ }^{29}$ Considering a pair of interacting chains, the results showed that there are not only intrachain excitons, but also interchain excitons. By an intrachain exciton we mean a structure that contains, in a single chain, an electron coupled to a hole, while an interchain exciton consists of a bound state of a positive polaron on one chain and a negative polaron on the neighboring chain. Also, the results suggest that the creation efficiency of interchain excitons increases by increasing the 
interchain coupling, while that of intrachain excitons decreases. However, these studies were performed considering weak interchain coupling even in the case of simulations of interchain excitons. Here, we describe the dissociation process in a single chain, leading to polaron formation; and the recombination process in two neighboring chains, which is explained as a recombination of polarons generating interchain excitons. The above-mentioned discussions in the literature show that these processes are still not fully understood. The dependence of the recombination process in a single chain and on two neighboring chains on the interchain coupling and the difference between these two process must be further clarified.

A feature usually not appropriately described in the literature is the temperature influence on the decay process of charge carriers. Although thermal effects are known to be important to the charge carriers creation mechanism, recent theoretical and experimental studies suggest that the photogeneration creation time is temperatureindependent. ${ }^{25-28,30-32}$ Besides, experimental evidence suggests that the dynamical decay of transient photoconductivity is temperature-independent. ${ }^{33}$ These results suggest that the contribution of the localized charge carriers (polarons) to the photocurrent is predominant at higher time scales. ${ }^{34}$

In the present work, we performed a systematic numerical investigation of charge carrier recombination as well as exciton dissociation in organic semiconductors. Both the exciton dissociation and charge carrier creation mechanisms were analyzed in $\pi$-conjugated polymer chains subjected to different thermal regimes. Beginning with a polaron-exciton initial state, under the influence of thermal effects, we observed a relaxation process that gives rise to a localized, polaron-type charge carrier. In the case of interchain excitons, it was obtained that polaron recombination process in two neighboring chains does not generate an usual exciton state. The importance of these results lies in the possibility of increasing the efficiency of intrachain exciton creation leading to an improvement of the photocurrent on the femtosecond time scale.

An Ehrenfest molecular dynamic simulation was performed by using a tight binding with a lattice relaxation taken into account in first-order approximation. Temperature effects were included by means of a canonical Langevin equation. Calculations considering terms of electron-electron repulsion as Hubbard and extended Hubbard terms were also performed here. Nevertheless, the addition of these terms did not qualitatively change the results of the cases studied. We interpret this as an indication that the relaxation brought by the electron-lattice coupling and the temperature inclusion contain the most important effects to describe these systems.

Since our goal is to investigate the intrachain polaronexciton relaxation and subsequent interchain polaron recombination, we considered two different cases: (i) a single chain and (ii) two coupled chains. In the latter, a hopping integral term was added in order to take interchain effects into account. In the former case, it was simulated the dynamics of a polaron-exciton in a cis-polyacetylene chain under the presence of different thermal regimes in order to observe the monomolecular exciton dissociation. To simulate recombination in two chains, it is necessary to consider a oppositely charged carrier on each chain. We considered a system in which a chain with a positive polaron is parallel to a second chain containing a negative polaron. At a certain point, the interaction between chains is turned on, thus allowing the recombination process to occur.

\section{METHODOLOGY}

We choose a polyacetylene chain in cis configuration ${ }^{35,36}$ to study the effect of temperature on a conjugated polymer chain of finite length. The Hamiltonian model that we use in this paper is $H=H_{1}+H_{2}+H_{\text {int }}$, where $H_{1}$ and $H_{2}$ are SSHtype Hamiltonians ${ }^{37}$ and have the following form:

$$
\begin{aligned}
H_{j}= & -\sum_{n, s} t_{j n, n+1}\left(C_{j n+1, s}^{\dagger} C_{j n, s}+H . c\right) \\
& +\sum_{n} \frac{K}{2} y_{j n}^{2}+\sum_{n} \frac{p_{j n}^{2}}{2 M} .
\end{aligned}
$$

Here, $j$ indexes the chains and $n$ indexes their sites. The operator $C_{j n+1, s}^{\dagger}$ creates and the operator $C_{j n, s}$ annihilates a $\pi$ electron at the $n$th site with spin $s$ in the $j$ th chain; $K$ is the harmonic constant due to the $\sigma$ bonds. $M$ is the mass of a $\mathrm{CH}$ group. $y_{j n} \equiv u_{j n+1}-u_{j n}$, where $u_{j n}$ is the lattice displacement of the atom at $n$th site along the $j$ th chain. $p_{j n}$ is the momentum conjugated to $u_{j n}$, and the quantity $t_{j n, n+1}$ is the hopping integral, given by

$$
t_{j n, n+1}=\left[1+(-1)^{n} \delta_{0}\right]\left(t_{0}-\alpha y_{j n}\right),
$$

with $t_{0}$ being the hopping integral of a $\pi$-electron between nearest neighbor sites in the undimerized chain and $\delta_{0}$ is the Brazovskii-Kirova symmetry breaking term, used to take the cis symmetry of the polymers into account.

The coupling between the two chains is due the interchain interaction described by $H_{\text {int }},{ }^{30}$

$$
H_{i n t}=-\sum_{n, s}^{q} t_{\perp}\left(C_{1 n, s}^{\dagger} C_{2 n, s}+C_{2 n, s}^{\dagger} C_{1 n, s}\right),
$$

where $t_{\perp}$ is the transfer integral between sites labeled by the same $n$ on different chains. The parameters chosen in this letter are $t_{0}=2.5 \mathrm{eV}, t_{\perp}=0.2 \mathrm{eV}, M=1349.14 \mathrm{eV}$ $\times \mathrm{fs}^{2} / \AA^{2}, K=21 \mathrm{eV} \AA^{-2}, \delta_{0}=0.05$ and $\alpha=4.1 \mathrm{eV} \AA^{-1}$. These values have a good track record ${ }^{27,28,30-32,34}$ and the results obtained using these parameters are expected to be valid for conjugated polymers, in general.

In order to solve this problem numerically, we must first obtain an initial solution for the polymer chains, i.e., we must obtain a stationary state fully self-consistent with the degrees of freedom of the system formed by the combination of all electrons and the lattice. The time evolution of the lattice configuration is then determined by the equations of motion. The equation of motion for the electronic wave function $\psi_{k, s}$ is the solution of the time-dependent Schrödinger equation,

$$
\begin{aligned}
i \hbar \dot{\psi}_{j k, s}(n, t)= & -t_{j n, n+1} \psi_{j k, s}(n+1, t) \\
& -t_{j n-1, n}^{*} \psi_{j k, s}(n-1, t)-t_{\perp} \psi_{\hat{j} k, s}(n, t),
\end{aligned}
$$


where $j$ and $\hat{j}$ index the chain running from 2 to 1 and 1 to 2 , respectively.

The equation of motion that describes the site displacement used in the temporal evolution of the lattice is determined within a classical approach, ${ }^{35}$

$$
\begin{aligned}
F_{n}(t)= & M \ddot{u}_{n}=-K\left[2 u_{n}(t)-u_{n+1}(t)-u_{n-1}(t)\right] \\
& +\alpha\left[B_{n, n+1}-B_{n-1, n}+B_{n+1, n}-B_{n, n-1}\right],
\end{aligned}
$$

in which we removed the $j$-index to simplify the notation. Here,

$$
B_{n, n^{\prime}}=\sum_{k, s}{ }^{\prime} \psi_{k, s}^{*}(n, t) \psi_{k, s}\left(n^{\prime}, t\right)
$$

is the term that couples the electronic and lattice problems. The primed summation represents a sum over the occupied states only. In the context of Koopmans' theorem, we simulate an excitation from the highest occupied molecular orbital (HOMO) to the lowest unoccupied molecular orbitals (LUMO) by changing the occupation number in the above sum.

We introduce the expansion,

$$
\psi_{k}\left(t_{j}\right)=\sum_{l} C_{l k} \phi_{l}\left(t_{j}\right)
$$

with $C_{l k}=\left\langle\phi_{l} \mid \psi_{k}\right\rangle$, where $\left\{\phi_{l}\right\}$ is the set of eigenfunction of the electronic Hamiltonian at a given time $t_{j}$. The obtained solution of the time-dependent Schrödinger equation is written as $^{32}$

$$
\begin{aligned}
\psi_{k, s}\left(n, t_{j+1}\right)= & \sum_{l}\left[\sum_{m} \phi_{l, s}^{*}\left(m, t_{j}\right) \psi_{k, s}\left(m, t_{j}\right)\right] \\
& \times \exp \left(-i \frac{\varepsilon_{l} \Delta t}{\hbar}\right) \phi_{l, s}\left(n, t_{j}\right),
\end{aligned}
$$

where $\left\{\varepsilon_{l}\right\}$ represents the eigenvalues of the electronic part of the Hamiltonian at a given time $t_{j}$.

Since our model assumes a classical treatment of the lattice part of the system, it is possible to make use of the Langevin approach to consider thermal effects. We choose a white stochastic signal $\zeta(t)$ as the fluctuation term, i.e., a signal with the following properties: $\langle\zeta(t)\rangle \equiv 0$ and $\left\langle\zeta(t) \zeta\left(t^{\prime}\right)\right\rangle$ $=B \delta\left(t-t^{\prime}\right)$. A Stokes-like dissipation term is also included in this formalism. Therefore, Eq. (5) is modified to

$$
M \ddot{u}_{n}=-\gamma \dot{u}_{n}+\zeta(t)+F_{n} \equiv \tilde{F}_{n}(t) .
$$

The relationship between $\zeta, \gamma$, and the temperature $T$ of the system is given by the fluctuation-dissipation theorem,

$$
B=2 k_{B} T \gamma M \text {. }
$$

It is important to note that this approach of considering thermal effects in conducting polymers has been used before in the literature ${ }^{31,32}$ and has good track record.

\section{RESULTS}

Here, we present results obtained for temperatures ranging from $0 \mathrm{~K}$ to $50 \mathrm{~K}$. For all simulations we considered 100 site chains, subjected to periodic boundary conditions. To pro- vide a better visualization of the charge density results, we define an order parameter as follows:

$$
\bar{\rho}(t)=1-\frac{\rho_{n-1}(t)+2 \rho_{n}(t)+\rho_{n+1}(t)}{4} .
$$

In order to simulate excitons and polarons in the systems, we proceeded analogously to other studies of quasi-particle formation in this type of systems, ${ }^{26-28}$ i.e., we consider that an intrachain polaron-exciton arises from a HOMO to LUMO+1 photoexcitation in the single molecule system, whereas an interchain polarons emerges from a HOMO to LUMO excitation in a system formed by two chains. ${ }^{32}$ These choices match the spatial distribution of the molecular orbital associated with each level in the electronic energy structure. That means the LUMO +1 orbital is mainly the first chain in the former case, and the LUMO orbital is in the second chain in the other case. We considered only singlet excitons in the present simulations through changing the occupation number of spin up states in the unrestricted spin-orbital method used. Actually, spin triplet excitations could display different behaviors, as seen in Ref. 38.

Figure 1 shows the time evolution of charge density of a single chain at $0 \mathrm{~K}$ (Fig. 1(a)) and $50 \mathrm{~K}$ (Fig. 1(b)). In both cases, the system initially finds itself in a bound state of halfpolarons-polaron-excitons-for both temperature regimes. It should be noted that, associated with Figure 1, there is a second parallel chain (not shown) that presents analogous

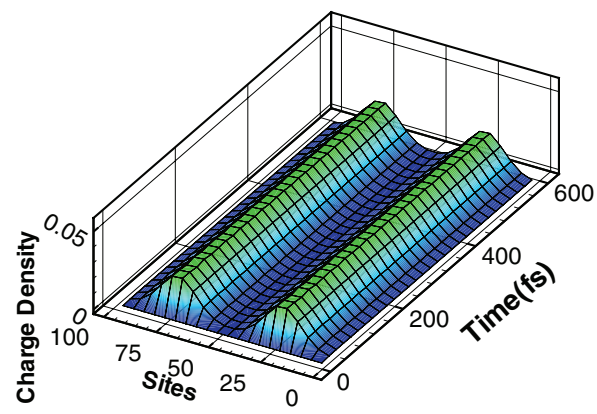

(a)

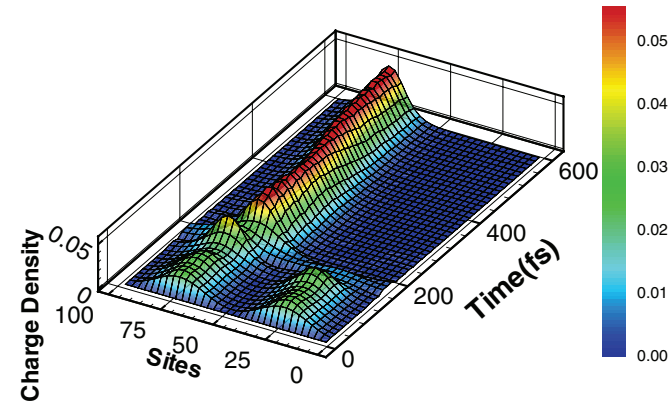

(b)

FIG. 1. The time evolution for single-chain charge density of the hole polaron at two different temperatures. In (a) there is no recombination, and in (b) the exciton dissociation occurs at $50 \mathrm{fs}$. 
behavior with the complementary electric charge. In Fig. 1(a), a polaron-exciton remains separated throughout the entire simulation. In the presence of temperature, the degeneracy is broken and a dissociation of a polaron-exciton into a free electron polaron and a free hole polaron is observed at $\sim 200$ fs (Fig. 1(b)). In other words, without temperature the system has no reason whatsoever to choose a particular site where to create the polaron. With temperature, even the tiny random variation on the site positions furnish the spontaneous symmetry breaking mechanism. This process might be responsible for the creation of free charge carriers in organic semiconductors in this time scale. It is important to note that the charge carrier creation mechanism does not occur in the absence of temperature, thus suggesting that temperature effects have an important role in the processes of exciton dissociation. We performed simulations for other values of temperature and observed the same pattern of polaron-exciton dissociating into free polarons. The recombination time was not altered by changes in temperature.

In Figure 2, we show the time evolution of the charge density for a system composed of two parallel chains at $50 \mathrm{~K}$ : a chain bearing a negative polaron (chain 1) in Fig. 2(a) and a chain bearing a positive polaron (chain 2) in Fig. 2(b). Through the photoexcitation process described above, a polaron is formed in chain 1 , and, since there is a small coupling between these chains, chain 2 relaxes generating a symmetrical polaron of charge $-e$ at the same time. Notice that charge neutrality is preserved upon photoexcitation. In Figure 2, we can observe that there is a relaxation time

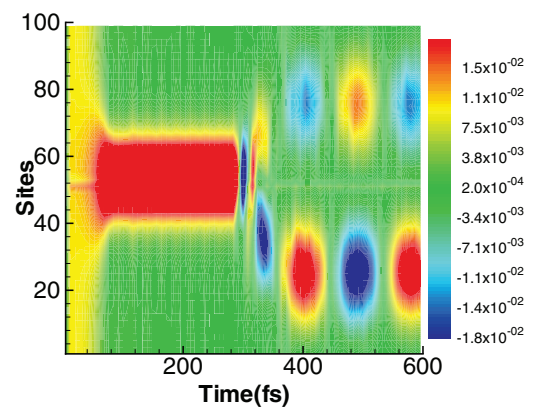

(a)

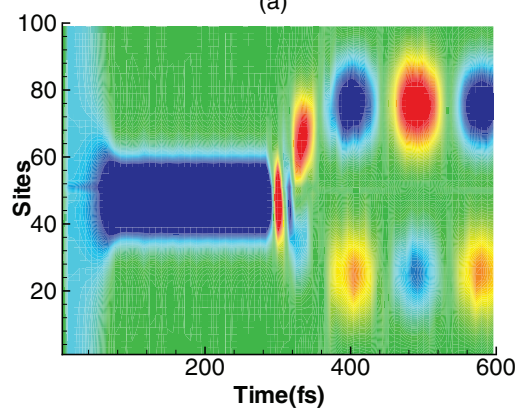

(b)

FIG. 2. Charge density time evolution at $50 \mathrm{~K}$. In (a), a polaron is formed with charge $(-e)$, and in (b) a polaron is formed with charge $e$. Notice that charge neutrality is preserved upon photoexcitation. of about $80 \mathrm{fs}$ before a polaron is formed. Note that the difference in the charge relaxation time presented in Figures 1 (b) and 2 is due to the differences in the photoexcitation process: the former is a HOMO to LUMO+1 and the later is a HOMO to LUMO excitation. This creation process is well discussed in Ref. 30 and our results are in agreement with both experimental and theoretical evidences..$^{24,25}$

The interaction between chains is turned on $280 \mathrm{fs}$ after the beginning of the simulation. Immediately after the coupling between chains is set, the polaron located in chain 1 combines with the other polaron located in chain 2 , characterizing an intermolecular recombination. In Figure 2, we can see that after the dissociation of the negative polaron, chain 1 has predominantly positive charge spread all over it. In the case of chain 2, that started bearing a positive polaron, a negative charge is found to be delocalized over this chain. Figure 2 shows that, after the annihilation of polarons in the two chains, there is a formation of an oscillating dipole between the two chains associated with two oscillating excitons. These two alternating excitons are coupled to phonon modes. ${ }^{39}$ The charge density oscillates at a frequency in the infrared region $\left(\sim 10^{13} \mathrm{~Hz}\right)$.

The yield of intrachain exciton formation decreases with the increase of interchain coupling, as suggested in Ref. 28. Moses et al. ${ }^{24}$ also suggested that bimolecular recombination is sensitive to the strength of the interaction and that charge carrier decay rate is weakly dependent on temperature. ${ }^{21}$ In summary, the single chain exciton dissociation process is responsible for the creation of free charge carriers that are annihilated in a fast bimolecular recombination process. The description of the charge-carrier dynamics of generation and decay in organic semiconductors cannot be observed in the absence of temperature.

In Figure 3, we present the polaron energy levels as a function of simulation time. This result corresponds to the simulation performed for two coupled chains shown in Figure 2. The transient state observed until $200 \mathrm{fs}$ corresponds to the formation of two initial polarons. After this time, characteristic levels of a polaron are formed inside the gap and remains there until 280 fs. Note the double degeneracy of the energy levels. At this time, the coupling between is set and bimolecular recombination process occurs. Polarons

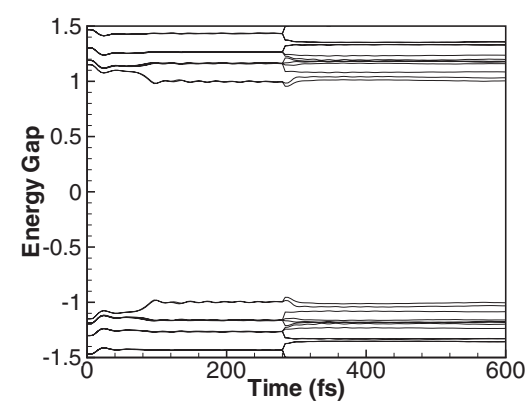

FIG. 3. The band structure of the system at the gap with the valence and conduction bands energy levels at $50 \mathrm{~K}$. Note the typical polaron energy level formation inside the gap at $\sim 100 \mathrm{fs}$. 
are annihilated and their corresponding energy levels that are inside the gap changes. As a consequence, a band structure of an organic semiconductor without these quasi-particles is obtained. The relaxation time obtained here is in agreement with the results by Soci et al. ${ }^{25}$ An energy level fluctuation can also be observed in Figure 3. This fluctuation is due to thermal effects, since this fluctuation was not observed for the $0 \mathrm{~K}$ case.

\section{CONCLUSIONS}

In this paper we studied the processes of intrachain exciton dissociation and interchain charge carrier recombination in organic semiconductor polymers. Particularly, we analyze the process of single-molecule polaron-exciton dissociation and polaron recombination in two parallel chains in these materials, under different temperature regimes. We performed simulations using a semi-empirical tight binding model with relaxation in a first order expansion, modified to include temperature effects. In order to investigate the intrachain polaronexciton dissociation and subsequent interchain polaron recombination, we considered two different cases: (i) a single chain and (ii) two coupled chains. In the case of two coupled chains, we observed that polaron recombination does not generate an usual exciton. Rather, this type of recombination leads to an exciton associated with an oscillating dipole between the two chains. Besides, the polaron recombination happens immediately after the coupling between chains is turned on. For single chains, our results show that in the absence of temperature, the excitons remain stable, not generating free carriers. This fact can be attributed to the high level of symmetry that systems, not thermally perturbated, present. When temperature effects are considered, we observed that on single chain, a polaron-exciton suffers spontaneous dissociation forming a free polaron. These results are in good agreements with experimental data ${ }^{33}$ and previous theoretical simulations performed for weakly coupled chains bearing a polaron-exciton. ${ }^{32}$ We concluded that temperature effects are important in the processes of recombination, particularly in the single-molecule polaron-exciton dissociation process, which does not occur without the influence of temperature. It was also observed that the increase of temperature does not influence the time interval between creation and decay of charge carriers. This suggests that the efficiency of organic semiconductors is independent of temperature, which is also in agreement with the experimental data. ${ }^{21,23}$

\section{ACKNOWLEDGMENTS}

The authors gratefully acknowledge the financial support from the Brazilian Research Councils CNPq, CAPES, and FINATEC.
${ }^{1}$ J. W. Yoo, C. Y. Chen, H. W. Jang, C. W. Bark, V. N. Prigodin, C. B. Eom, and A. J. Epstein, Nature Mater. 9, 638 (2010).

${ }^{2}$ V. A. Dediu, L. E. Hueso, I. Bergenti, and C. Talian, Nature Mater. 8, 707 (2009).

${ }^{3}$ P. Matyba, K. Maturova, M. Kemerink, N. D. Robinson, and L. Edman, Nature Mater. 8, 672 (2009).

${ }^{4}$ P. A. Bobbert, T. D. Nguyen, W. Wagemans, F. W. A. van Oost, B Koopmans, and M. Wohlgenannt, Synth. Met. 120, 223 (2010).

${ }^{5}$ V. N. Prigodin and A. J. Epstein, Synth. Met. 160, 244 (2010).

${ }^{6}$ T. Sakanoue and H. Sirringhaus, Nature Mater. 9, 737 (2010).

${ }^{7}$ S. Reineke, F. Lindner, G. Schwartz, N. Seidler, K. Walzer, B. Lüssem, and K. Leo, Nature (London) 459, 234 (2009).

${ }^{8}$ T. D. Nguyen, G. Hukic-Markosian, F. Wang, L. Wojcik, X. Li, E. Ehrenfreund, and Z. V. Vardeny, Nature Mater. 9, 345 (2010).

${ }^{9}$ H. J. Kim, M. J. Kim, H. D. Park, J. H. Lee, S. T. Nohd, Y. C. Lee, and J. J. Kim, Synth. Met. 160, 1994 (2010).

${ }^{10}$ T. D. Nguyen, Y. Sheng, J. Rybicki, G. Veeraraghavan, and M. Wohlgenannt, Synth. Met. 160, 320 (2010).

${ }^{11}$ F. Wang, C. G. Yang, E. Ehrenfreund, and Z. V. Vardeny, Synth. Met. 160, 297 (2010).

${ }^{12}$ B. N. Pal, B. M. Dhar, K. C. See, and H. E. Katz, Nature Mater. 8, 898 (2009).

${ }^{13}$ J. Rivnay, L. H. Jimison, J. E. Northrup, M. F. Toney, R. Noriega, S. F. Lu, T. J. Marks, A. Facchetti, and A. Salleo, Nature Mater. 8, 952 (2009).

${ }^{14}$ H. Yan, Z. Chen, Y. Zheng, C. Newman, J. R. Quinn, F. Dotz, M. Kastler, and A. Facchetti, Nature (London) 457, 679 (2009)

${ }^{15}$ M. R. Lee, R. D. Eckert, K. Forberich, G. Dennler, C. J. Brabec, and R. A. Gaudiana, Science 324, 232 (2009).

${ }^{16}$ R. A. Afre, Y. Hayashi, and T. Soga, Synth. Met. 160, 779 (2010)

${ }^{17}$ M. Skompska, Synth. Met. 160, 1 (2010).

${ }^{18}$ Y. Zhang, G. Hukic-Markosian, D. Mascaro, and Z. V. Vardeny, Synth. Met. 160, $262(2010)$

${ }^{19}$ S. Singha, Y. Zhang, and Z. V. Vardeny, Synth. Met. 60, 311 (2010).

${ }^{20}$ W. P. Su, J. R. Schrieffer, and A. J. Hegger, Phys. Rev. B 22, 2099 (1980).

${ }^{21}$ B. Kippelen and J. L. Brdas, Energy Environ. Sci. 2, 251 (2009).

${ }^{22}$ D. Moses, H. Okumoto, C. H. Lee, and A. J. Heeger, Phys. Rev. B 54, 4748 (1996)

${ }^{23}$ V. Gulbinas, D. Hertel, A. Yartsev, and V. Sundstrom, Phys. Rev. B 76, 235203 (2007).

${ }^{24}$ D. Moses, A. Dogariu, and A. J. Heeger, Synth. Met. 116, 19 (2001).

${ }^{25}$ C. Soci, D. Moses, and A. J. Heeger, Synth. Met. 153, 145 (2005).

${ }^{26}$ C. Soci, D. Moses, Q. Xu, and A. Heeger, Phys. Rev. B 72, 245204 (2005)

${ }^{27}$ Y. Meng, X. J. Liu, B. Di, and Z. An, J. Chem. Phys. 131, 244502 (2009).

${ }^{28}$ Y. Meng, B. Di, X. J. Liu, Z. An, and C. Q. Wu, J. Chem. Phys. 128, 184903 (2008).

${ }^{29}$ Z. G. Yu, M. W. Wu, X. S. Rao, X. Sun, and A. R. Bishop, J. Phys. Condens. Matter 8, 8847 (1996).

${ }^{30}$ Y. Meng and Z. An, Eur. Phys. J. B 74, 313 (2010)

${ }^{31}$ P. H. de Oliveira Neto, W. F. da Cunha, R. Gargano, and G. M. e Silva, J. Phys. Chem. A 113, 14975 (2009).

${ }^{32}$ P. H. de Oliveira Neto, W. F. da Cunha, and G. M. e Silva, Eur. Phys. Lett. 88, 67006 (2009)

${ }^{33}$ S. H. Kim, T. Zyung, H. Y. Chu, L. M. Do, and D. H. Wang, Phys. Rev. B 61, 15854 (2000)

${ }^{34}$ P. H. de Oliveira Neto, W. F. da Cunha, L. F. Roncaratti, R. Gargano, and G. M. e Silva, Chem. Phys. Lett. 493, 283 (2010).

${ }^{35}$ X. C.D. Moses, C. Soci, and A. P. Ramirez, Phys. Rev. Lett. 97, 067401 (2006).

${ }^{36}$ J. Day, S. Subramanian, J. E. Anthony, Z. Lu, R. J. Twieg, and O. Ostroverkhova, J. Appl. Phys. 103, 123715 (2008).

${ }^{37}$ W. P. Su, J. R. Schrieffer, and A. J. Hegger, Phys. Rev. Lett. 33, 1698 (1979).

${ }^{38}$ Z. Sun, D. Liu, S. Stafstrn, and Z. An, J. Chem. Phys. 134, 044906 (2011).

${ }^{39}$ Z. An, C. Q. Wu, and X. Sun, Phys. Rev. Lett. 93, 216407 (2004). 


\section{APPENDIX \\ PAPER IV}

\section{Impurity Effects and Temperature Influence on the Exciton Dissociation Dynamics in Conjugated Polymers}

Luiz Antonio Ribeiro Junior

Wiliam Ferreira da Cunha

Pedro Henrique de Oliveira Neto

Ricardo Gargano

Geraldo Magela e Silva

Chem. Phys. Lett., 580, 108-114 (2013) 


\title{
Impurity effects and temperature influence on the exciton dissociation dynamics in conjugated polymers
}

\author{
Luiz Antonio Ribeiro, Wiliam F. da Cunha, Pedro H. Oliveira Neto, R. Gargano*, Geraldo M. e Silva \\ Institute of Physics, University of Brasília, Brasília, Brazil
}

\section{A R T I C L E I N F O}

\section{Article history:}

Received 10 May 2013

In final form 20 June 2013

Available online 5 July 2013

\begin{abstract}
A B S T R A C T
We investigate temperature effects on exciton dissociation dynamics in conjugated polymer systems. Using a modified version of the tight-binding Su-Schrieffer-Heeger model, the dissociation is studied under the influence of impurity effects with a nonadiabatic evolution method. Our results show that temperature effects reduce the critical electric field for the exciton dissociation. In the small temperature regime, the exciton is not trapped by the impurity and it is observed to perform a random walk, a fact not observed in the absence of temperature. This letter might enlighten the description of electroluminescence yields and charge transport efficiency in organic based electronic devices.
\end{abstract}

(c) 2013 Elsevier B.V. All rights reserved.

\section{Introduction}

Conjugated polymers are currently being used as active materials in various optoelectronic devices such as Organic Solar Cells (OSCs) [1], Thin-Film Transistors (TFTs) [2], and Polymer LightEmitting Diodes (PLEDs) [3]. Singular characteristics, such as light weight, flexibility, versatility of chemical synthesis, and low cost, make them commercially interesting and thus lead to considerable effort in the understanding of how to develop better materials and more efficient devices for the aforementioned class of systems.

In contrast to the conventional inorganic conductors, the fact that conjugated polymer are quasi-one-dimensional materials leads to the novel property of its lattice structure being easily distorted to form self-trapped elementary excitations. This can be accomplished either by charge injections or by a photoexcitation mechanism, and results on the induction of self-localized electron states, such as excitons [4]. In conjugate polymers, an exciton is a bound electron-hole pair state formed due to the strong electronlattice interactions. It is known that the efficiency of PLDEs and OSCs is related to the exciton dissociation mechanism. A PLED normally consists on a luminescent and a conducting conjugate polymer layer, introduced between two metal electrodes. Electrons and holes are injected from the electrodes into the polymer layer; as a result, this process induce self-localized electron states, which leads to the exciton formation.

In these materials excitons are generally considered to be more strongly localized than excitons in three-dimensional semiconductors, especially because in the former the exciton is substantially confined to a single polymer chain [5]. A schematic energy-level

\footnotetext{
* Corresponding author. Fax: +55 (61) 33072363

E-mail address: gargano@unb.br (R. Gargano).
}

diagram for a PLED under forward bias, that represents the exciton dissociation mechanism in this material, is shown in Fig. 1(a). This phenomenon is accomplished as follows: First, electrons and holes are injected from negative (anode) and positive (cathode) electrodes, respectively (step 1 , in the figure). Following, electrons and holes capture one another within the emissive polymer film, and form neutral bound excited state (step 2). Finally, a radiative decay of the excited electron-hole state produced by the recombination process take place (step 3 ). The spin wavefunction of the exciton, formed from the two spin- $\frac{1}{2}$ electronic charges, can be either singlet $(S=0)$ or triplet $(S=1)$. The radiative emission (fluorescence) is from the singlet only, and when the exchange energy is large, cross-over from triplet to singlet is unlikely to happen, so that triplet excitons do not produce light emission other than by indirect processes such as phosphorescence or by triplet-triplet annihilation [5,6]. In PLEDs The emissive polymer layer generally is formed by a Poly ( $p$-phenylenevinylene) (PPV) film, which have high photoluminescence yields $[7,8]$. The hole transport layer (conducting polymer) widely used in this devices is the Polyaniline (PANI:PPS). Indium-tin Oxide (ITO) is used as the hole-injecting electrode and a magnesium-silver alloy plays the role of the electron-injecting electrode. Considering the OSCs devices, the photovoltaic effect also involves the generation of electron and hole pairs and their subsequent collection at the opposite electrodes [9].

A key aspect in the physics of organic compared to inorganic semiconductors is the difference on the nature of the optically excited states. Whereas in inorganic materials, the production of free charge is carried out directly, the absorption of a photon in organic materials causes a delocalization of these sates, which leads to the formation of an exciton due to the strong electron-lattice interactions. The organic exciton binding energy is naturally large, on the order of or larger than $500 \mathrm{meV}$. This binding energy represent 

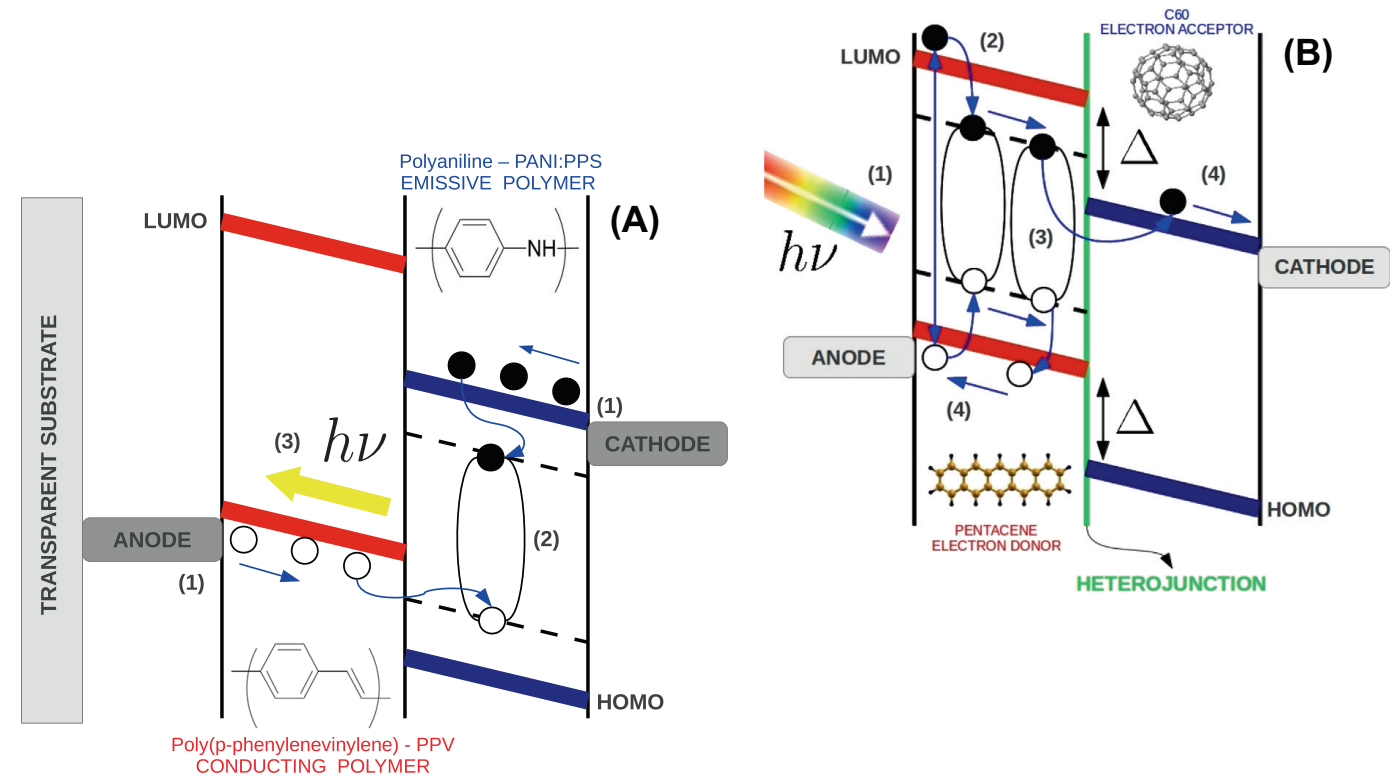

Fig. 1. Schematic energy-level diagram (a) for a PLED and (b) an OSC.

twenty times or more the thermal energy at room temperature, $k_{B} T(300 K)=26 \mathrm{meV}$ to be compared with a few $\mathrm{meV}$ in the case of inorganic semiconductors [9].

A schematic energy-level diagram for a OSC under illumination, that represents the exciton dissociation mechanism, is shown in Figure 1(b). As a first step (1) the photons are absorbed with an average photon energy larger than the optical band gap on both sides of the heterojunction. Following, occurs the thermalization and the formation of excitons (step 2). The next stage is the excitons diffusion to the heterojunction (3). Finally (4) these structures are dissociated and transfer an electron (hole) into the acceptor (donor) layer. The $\Delta$ arrows denote the energy offsets between the ionization potential values (HOMO energies), of the donor molecular layer, and electron affinities (LUMO energies) of the acceptor molecular layer. Figure 1(b) presents a OSC whose conducting polymer layer is formed by a $C_{60}$ film, which have high electron affinity molecules and favors the rapid exciton dissociation, thus resulting in high-power conversion efficiencies [10]. The hole transport layer widely used in these devices is a Pentacene film. The hole-injecting electrode and the electron-injecting electrode are the same in the PLEDs devices.

The above discussions shows that the optical absorption in materials based on conjugated polymers does not lead directly to free electron and hole carriers that could readily generate electrical current. Consequently, to generate current in OSCs, the excitons must first dissociate into free charges. Also, excitons are of great importance in conjugated polymers especially in photoluminescence (PL) emission resultant from the radiative decay of the singlet excitons in PLEDs [11-16]. Furthermore, it has been generally accepted that the temperature effcts are of fundamental importance on monomolecular recombination of mixed states composed by polarons and excitons $[17,18]$. Thus, the exciton dissociation in the presence of thermal effects are believed to be of fundamental importance for PLEDs. However, studies that take into account this physical picture remains not well described in the literature.

Another question of major importance is the role played by impurity effects on exciton dissociation in conjugate polymers.
Zhao et al., using an extended version of SSH model modified to include impurity interactions and the Brazoviski-Kirova symmetry breaking term, investigated the dynamical process of exciton dissociation in the presence of an external electric field [19]. Their results have shown that, under the action of impurities, the stability as well as the effective mass of the exciton is reduced. Also, the field required to dissociate the excitons depends sensitively on the strength of the impurity potential. As the impurity potential strength increases, the dissociation field effectively decreases. In the absence of an impurity in the polymer lattice, the exciton dissociation occurs with an electric field regime of $7.9 \mathrm{mV} / \mathrm{A}$. Using an impurity strength of $0.25 \mathrm{eV}$, the critical electrical field decreases to $5.7 \mathrm{mV} / \AA$. Particularly, understanding the effects of the impurity interactions in the presence of thermal effects on exciton dissociation in conjugated polymer systems, is an issue that is believed to be crucial for the design of more efficient devices with respect to the electroluminescence. Thus, this point requires a better phenomenological description.

A relevant theoretical study carried out by Stafström have shown that the exciton dissociation depends crucially on the strength of the applied electric field [20]. An extended version of the SSH model which describes three-dimensional structures was applied to dynamical studies of excited system consisting of two coupled PPV chains. The results showed that the exciton dissociation were not accomplished for field strength lower than $2.0 \mathrm{mV} /$ Åwhereas at $2.8 \mathrm{mV} /$ Åcharge separation was observed to occur within $250 \mathrm{fs}$. The process of charge separation involves a redistribution of the electrons among the energy levels. This process is field induced but is also strongly dependent on the lattice energy (temperature) and the excitation energy. An increase in these two types of energies both lead to a shortening of the separation time. It is important to remark that all these studies have focused on specific cases with idealized conditions. A theory that widely holds for real materials needs further elements by addressing some realistic effects such as temperature and impurity effects. Also, from these works, we can see that all the results for the exciton dissociation were not fully described, so that further investigation, that take into account temperature effects is needed. 
In this letter, a systematic numerical investigation of exciton dissociation is performed in a cis-polyacetylene chain in terms of a nonadiabatic evolution method $[21,22]$. The charge separation process of the exciton is investigated on a conjugated polymer chain subjected to different field strengths, impurity interactions, and temperature effects. An Ehrenfest Molecular Dynamics is performed by using a one-dimensional tight-binding model including lattice relaxation. This extended version of the SSH model is used to include external electric fields and Brazoviski-Kirova symmetry breaking terms. The aim of this letter is to give a physical picture of the singlet exciton dissociation in conjugated polymers, when temperature and impurity effects are taken together into account, and contribute to the understanding of these important processes, that may provide guidance for improving the electroluminescence yield in PLDEs and charge transport efficiency in OSCs.

\section{Methodology}

A polyacetylene chain in cis configuration [23] was used to study the charge carrier stability under thermal effects in conjugated polymers. The SSH-type Hamiltonian [24] modified to include an external electric field and the Brazovskii-Kirova symmetry-breaking term used in this work has the following form:

$H=-\sum_{n, s}\left(t_{n, n+1} C_{n+1, s}^{\dagger} C_{n, s}+\right.$ h.c. $)+\sum_{n} \frac{K}{2} y_{n}^{2}+\sum_{n} \frac{p 2_{n}}{2 M}$,

were $n$ indexes the sites of the chain. The operator $C_{n, s}^{\dagger}\left(C_{n, s}\right)$ creates (annihilates) a $\pi$-electron state at the $n$th site with spin $s ; K$ is the harmonic constant that describes a $\sigma$ bond and $M$ is the mass of a $\mathrm{CH}$ group. The parameter $y_{n}$ is defined as $y_{n} \equiv u_{n+1}-u_{n}$ where $u_{n}$ is the lattice displacement of an atom at the $n$th site. $p_{n}$ is the conjugated momentum to $u_{n}$ and $t_{n, n+1}$ is the hopping integral, given by

$t_{n, n+1}=e^{-i \gamma \mathbf{A}(t)}\left[\left(1+(-1)^{n} \delta_{0}\right) t_{0}-\alpha y_{n}\right]$,

where $t_{0}$ is the hopping integral of a $\pi$-electron between nearest neighbor sites in the undimerized chain, $\alpha$ is the electron-phonon coupling, and $\delta_{0}$ is the Brazovskii-Kirova symmetry-breaking term, which is used to take the cis symmetry of the polymer into account. $\gamma \equiv e a /(h c)$, with $e$ being the absolute value of the electronic charge, $a$ is the lattice constant, and $c$ is the speed of light. The relation between the time-dependent vector potential $\mathbf{A}$ and the uniform electric field $\mathbf{E}$ is given by $\mathbf{E}=-(1 / c) \dot{\mathbf{A}}$. The parameters used here are $t_{0}=2.5 \mathrm{eV}, M=1349.14 \mathrm{eV} \times f \mathrm{~s}^{2} / \AA^{2}, K=21 \mathrm{eV} \AA^{-2}, \delta_{0}=0.05, \alpha=$ $4.1 \mathrm{eVA}^{-1}$ and a bare optical phonon energy $h \omega_{Q}=$ $h \sqrt{4 K / M}=0.16 \mathrm{eV}$. These values have been used in previous simulations and are expected to be valid for conjugated polymers in general $[17,25,26]$.

In order to solve these equations numerically, first a stationary state that is self-consistent with all degrees of freedom of the system (the lattice and electrons) is obtained. We begin by constructing the hamiltonian from an arbitrary $\left\{\mathrm{y}_{n}\right\}$ set of positions. By solving the time dependent Schrödinger equation a new set of coordinates $\left\{\mathrm{y}_{n}^{\prime}\right\}$ is obtained. Iterative repetitions of this procedure yields a self consistent initial state when $\left\{\mathrm{y}_{n}\right\}$ is close enough to the solution.

The next step is to perform the time evolution of the system through the use of the equations of motion. The electronic wave function is the solution of the time-dependent Schrödinger equation:

$i h \dot{\psi}_{k, s}(n, t)=-t_{n, n+1} \psi_{k, s}(n+1, t)-t_{n-1, n}^{*} \psi_{k, s}(n-1, t)$

where $k$ is the quantum number that specifies an electronic state. The equation of motion that describes the site displacement and provides the temporal evolution of the lattice is obtained in a classical approach $[23,24]$. This equation is written as

$$
\begin{aligned}
F_{n}(t)= & M \ddot{u}_{n} \\
= & -K\left[2 u_{n}(t)-u_{n+1}(t)-u_{n-1}(t)\right] \\
& +\alpha\left[B_{n, n+1}-B_{n-1, n}+B_{n+1, n}-B_{n, n-1}\right],
\end{aligned}
$$

where $F_{n}(t)$ represents the force on the $n$th site. Here,

$B_{n, n^{\prime}}=\sum_{k, s}^{\prime} \psi_{k, s}^{*}(n, t) \psi_{k, s}\left(n^{\prime}, t\right)$

is the term that couples the electronic and lattice solutions. The primed summation represents a sum over the occupied states. By introducing instantaneous eigenstates, the solutions of the timedependent Schrödinger equation can be expressed as [27]

$\psi_{k, S}\left(n, t_{j+1}\right)=\sum_{l}\left[\sum_{m} \phi_{l, s}^{*}\left(m, t_{j}\right) \psi_{k, s}\left(m, t_{j}\right)\right] \times e^{\left(-i \varepsilon_{l} \Delta t / h\right)} \phi_{l, S}\left(n, t_{j}\right)$.

$\left\{\phi_{l}(n)\right\}$ and $\left\{\varepsilon_{l}\right\}$ are the eigenfunctions and the eigenvalues of the electronic part for the hamiltonian at a given time $t_{j}$. Eq. 4 , which defines the evolution of system, may be numerically integrated as $[27,28,23]$

$u_{n}\left(t_{j+1}\right)=u_{n}\left(t_{j}\right)+\dot{u}_{n}\left(t_{j}\right) \Delta t$,

$\dot{u}_{n}\left(t_{j+1}\right)=\dot{u}_{n}\left(t_{j}\right)+\frac{F_{n}\left(t_{j}\right)}{M} \Delta t$

Hence, the electronic wave functions and the lattice displacements at the $(j+1)$ th time step are obtained from the $j$ th time step. At time $t_{j}$ the wave functions $\left\{\psi_{k, s}\left(i, t_{j}\right)\right\}$ can be expressed as a series expansion of the eigenfunctions $\left\{\phi_{l, s}\right\}$ at that moment:

$\psi_{k, s}\left(i, t_{j}\right)=\sum_{l=1}^{N} C_{l, k}^{s} \phi_{l, s}(i)$,

where $C_{l, k}^{s}$ are the expansion coefficients. The occupation number for eigenstate $\phi_{l, s}$ is

$\eta_{l, s}\left(t_{j}\right)=\sum_{k}{ }^{\prime}\left|C_{l, k}^{S}\left(t_{j}\right)\right|^{2}$.

$\eta_{l, s}\left(t_{j}\right)$ describes the redistribution of electrons among the energy levels.

It is possible to make use of the Langevin approach to take thermal effects into account $[29,30]$ by using a white stochastic signal $\zeta(t)$ as the fluctuation term, i.e., a signal with the following properties: $\langle\zeta(t)\rangle \equiv 0$ and $\left\langle\zeta(t) \zeta\left(t^{\prime}\right)\right\rangle=2 M k_{B} T \Gamma \delta\left(t-t^{\prime}\right)$. A Stokes-like dissipation term is also included in this formalism. Adding the stochastic and the damping force and rewriting the equation of motion, the following equation is obtained [17,31]

$M \ddot{u}_{n}=-\Gamma \dot{u}_{n}+\zeta_{n}(t)+F_{n}(t) \equiv \tilde{F}_{n}(t)$.

The relationship between $\zeta_{n}(t), \Gamma$, and the temperature $T$ of the system is given by the fluctuation dissipation theorem. The damping constant can be determined by low temperature lattice thermal conductivity measurements. The $\Gamma$ value used here have the same order of magnitude as expected from experimental data of Raman spectral line width in polydiacetylene $\left(\Gamma=0.01 \omega_{Q}\right)[32]$.

\section{Results and discussion}

One of the most fundamental steps, and yet the greatest challenge in the field of organic electronics is the generation of free charge carriers (electrons and holes) through the dissociation of excitons. The usual method for doing it is the application of external electric fields, but topological effects, temperature and other interactions obviously affects the process. The main interest of the present study is to discuss the interaction between temperature and its effect on the dissociation of excitons, particularly in what concerns 
critical electric fields. In order to do so, we considered 100 sites (monomers) cis-polyacetylene chains with and without impurities, so that the effect of this symmetry breaking could also be investigated. It should be noted that the relative symmetry of the polymer plays an important role over the charge transport mechanism. Nevertheless, since the most used polymers are of cis symmetry, we decided to carry out our calculations with this kind of systems. The reason to perform this kind of simulation is to achieve a good compromise between the simplicity of the model and the realistic physical conditions that affects the behavior of organic electronic devices.

In this sense, the first step on performing a specific study on temperature and electric field influence over excitons dissociation is to isolate the effects of these properties from the side effects the applications of these excitations usually has over the systems dynamics. For instance, a sudden application of an electric field over the chain might lead to a lattice disturbance that, on its turn, might destabilize the quasi-particles. In order to mitigate this kind of effect, the electric field is turned on by applying a smooth change of the potential vector strength:

$$
\mathbf{A}(t)= \begin{cases}0 & \text { if } t<0, \\ \left(-c E\left[t_{s}-\left(\frac{t_{s}}{\pi}\right) \sin \left(\frac{\pi t}{t_{s}}\right)\right]\right) / 2 & \text { if } 0 \leqslant t<t_{\text {off }}, \\ -c\left(t-\frac{t_{s}}{2}\right) & \text { if } t_{s} \leqslant t<t_{\text {off }}, \\ \left(-c E\left[t+t_{\text {off }}-t_{s}+\left(\frac{t_{s}}{\pi}\right) \sin \left(\frac{t_{s}}{\pi}\left(t-t_{\text {off }}+\pi\right)\right)\right]\right) & \text { if } t_{w} \leqslant t<t_{\text {off }}+t_{s}, \\ -c E t_{\text {off }} & \text { if }-c E t_{\text {off }}\end{cases}
$$

with $t_{w}=10 \mathrm{fs}$ being the width, $t_{\mathrm{s}}=1 \mathrm{fs}$ the electric field turn-on period, and $t_{\text {off }}=1000 \mathrm{fs}$ the time of the field being turned off. Figure 2 presents the quasi-adiabatic approach used to apply the several different electric fields in our chain. One can see that the transient necessary to obtain the final value is about $40 \mathrm{fs}$, a period after which the electric fields of 4.0, 4.5, 5.0, 5.5 and $6.0 \mathrm{mV} /$ Åare achieved. By adopting this procedure we can safely conduct our analysis after times greater than $40 \mathrm{fs}$ without incurring in errors due to effect of the application itself. A somewhat different analysis should be performed on the temperature effects. As already mentioned, we introduce the temperature in our model by means of the phenomenological approach of addressing a Langevin equation to simulate the sites random motion. Thus, the measuring of temperature is obtained through the classical equivalence $k_{B} T=M\left\langle v_{n}^{2}\right\rangle$. However, a special care must be taken on relating a given temperature value to a simulation at different times because the process of thermalization is not carried out instantaneously.
Figure 3 presents the thermalization curves for the target final temperatures of $50,100,150,200,250$ and $300 \mathrm{~K}$. It is clear, from the figure, that the higher is the desired temperature, the more time it takes for the system to thermalize into this regime. Despite this fact, and since we seek to perform a qualitative investigation on the temperature influence over the excitons dynamics, by analyzing Figure 3 we can use the practical value of $500 \mathrm{fs}$ in order to carry out our analysis. In other words, we believe that $500 \mathrm{fs}$ is a time that, in average, all the temperatures remains somewhat near the desired values. We can see that, by considering that we are missing by at most $20 \mathrm{~K}$, i.e., no more than $10 \%$. We are now in the position to effectively handle the main subject of the letter, i.e., the exciton dissociation in conjugate polymer systems. Here, an investigation of the exciton dissociation behavior as a function of temperature and electric fields as well as of whether or not a $0.25 \mathrm{eV}$ impurity is present is developed. It is important to note that this impurity value is chosen in order to perform a reasonable comparison with a recent theoretical study carried out without temperature effects [19]. We study the effect of all the electric fields of Figure 2 and all the temperatures of Figure 3, but choose to present only the most significant results in Figure 4.

Through all the simulations, an exciton is initially created at the 50th site and performs a random walk due to the temperature applied to the system. All the simulations lasts for $1000 \mathrm{fs}$, so that the aforementioned problems of considering times greater than $40 \mathrm{fs}$ due to electric field application and greater than 500 fs due to thermalization is not an issue, provided we focus on the suitable region of the figures. An interesting property common to all the simulations is the effective presence of the exciton structure since the beginning of the simulation, a fact that can be readily noted by simultaneously studying Figures 4(a) and (b): it is observed that the two oppositely charged structures in Figure $4-$ a hole-polaron and an electron-polaron - yield a single lattice distortion in Figure 4(a). Overall we have a neutral particle corresponding to a single lattice distortion presenting the collective behavior that characterizes itself as an exciton quasi-particle. Naturally, the time that each structure lasts (as well as its dynamic behavior) is highly dependent on the temperature regime, electric fields and impurities properties. This can be inferred from the differences of the Sections $1-3$ of Figure 4. As a matter of fact, what is done is to systematically vary our desired quantities, i.e., temperature and electric field, until the point of exciton dissociation. This way, we are able to obtain critical temperatures and, most important, critical electric fields to achieve charge separation under certain circumstances.

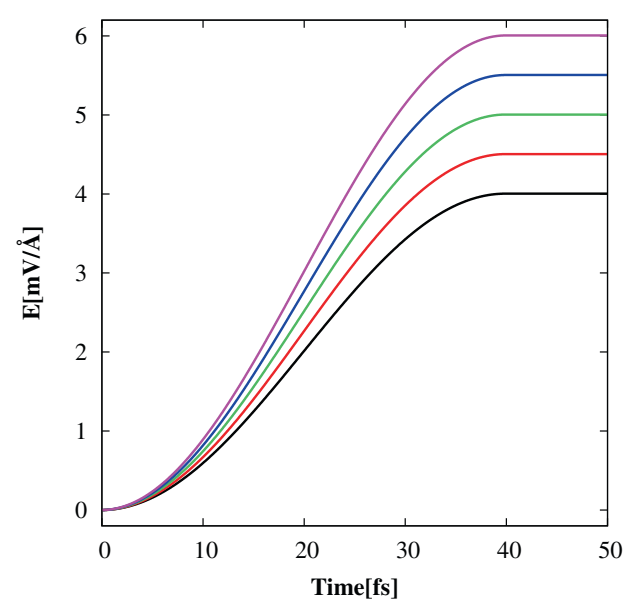

Fig. 2. Quasi-adiabatic application of 4.0, 4.5, 5.0, 5.5 and $6.0 \mathrm{mV} /$ Åelectric fields.

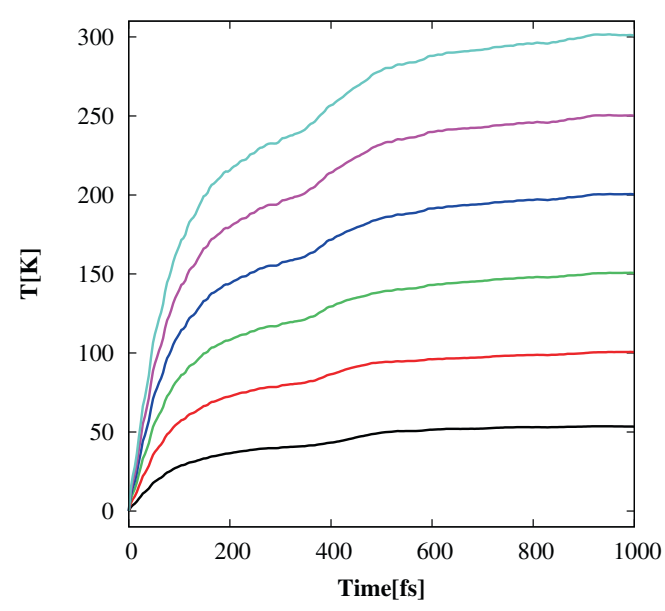

Fig. 3. Thermalization curves for $50,100,150,200,250$ and $300 \mathrm{~K}$. 

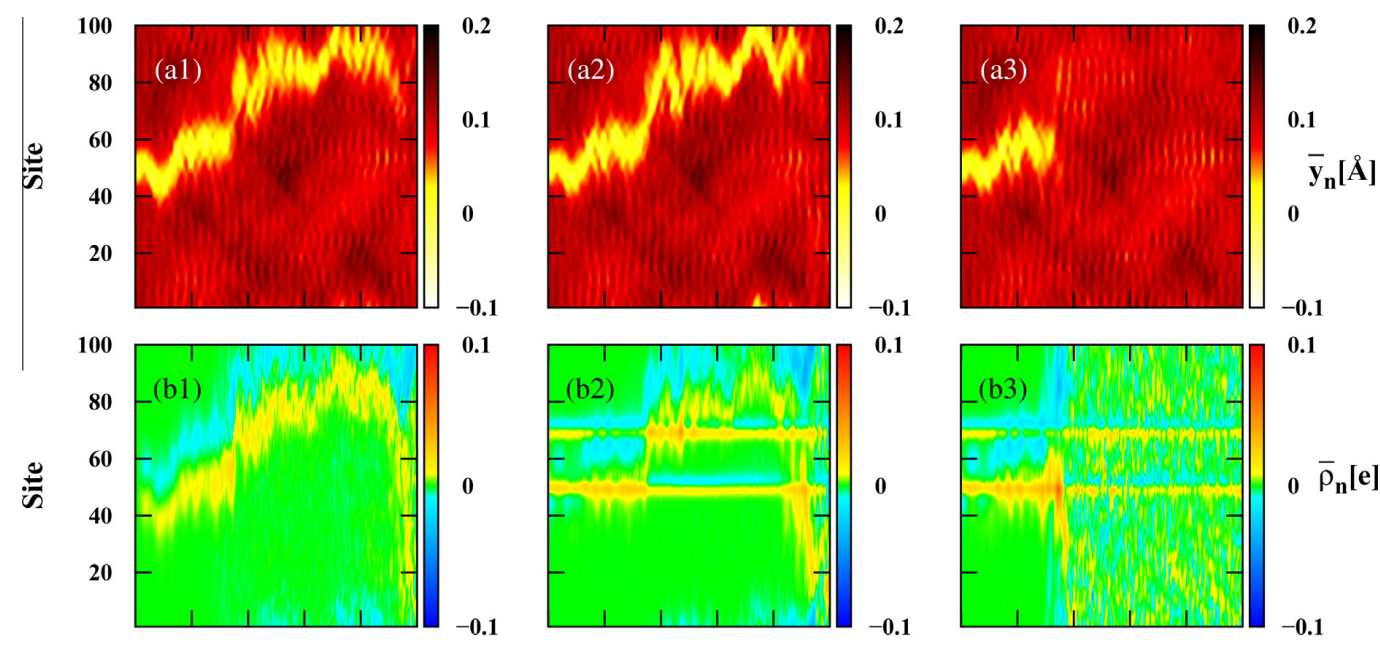

0.1

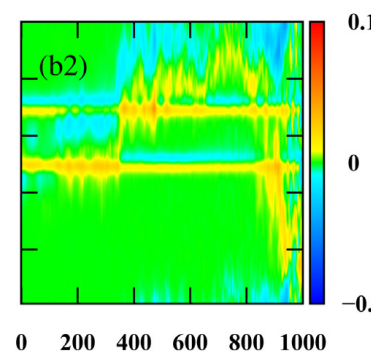

Time[fs]

Time[fs]

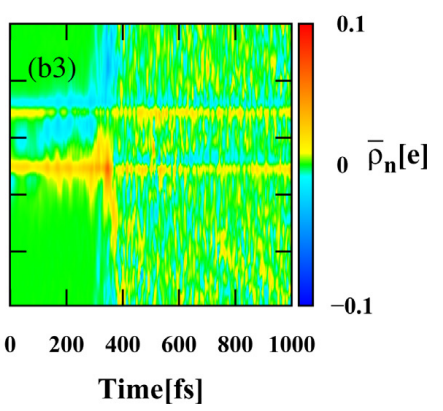

Fig. 4. (a) Lattice order paramete; (b) time evolution for the charge density for: 1 - Pristine chain at $300 \mathrm{~K}$ subjected to a $5.0 \mathrm{mV} /$ Åelectric field; $2-0.25 \mathrm{eV}$ impurity doped chain at $100 \mathrm{~K}$ subjected to a $6.0 \mathrm{mV} /$ Åelectric field and $3-0.25 \mathrm{eV}$ impurity doped chain at $300 \mathrm{~K}$ subjected to a $3.9 \mathrm{mV} /$ Åelectric field.

Figure 4 presents the results of the simulations whose parameters are those critical values. We begin by analyzing the first Section 1 of Figure 4. This section reports the results of an initially exciton placed in a $300 \mathrm{~K}$ polymer chain free of impurities and subjected to an electric field of $5.0 \mathrm{mV} / \AA$. One can note the exciton random walk owing to thermal effects throughout the simulation. The consistency of the polaron-exciton as a quasi-particle can be observed again by comparing Figures 4(a1) to (b1), where we can see that the movement of the lattice distortion corresponds to the movement of the two charged particles and thus of the neutral entity composed of this interaction. In other terms, the collective behavior that the system presents is preserved until around $900 \mathrm{fs}$. After this time, the electric field excitations as well those from the phonon modes created in the lattice, add up in the sense of leading to a destruction of the coupling between electron-polaron and hole-polaron. This is the desired phenomenon of exciton dissociation, that separates positive from negative charges, thus providing net charge for conduction and use in organic based devices. In this case, we observed a critical dissociation electric field of $5.0 \mathrm{mV} /$ Åfor a dissociation time of around $900 \mathrm{fs}$. Through the consideration of temperature we are able to observe the exciton dissociation about twice as fast as when no temperature is considered [20].

Besides obtaining the exciton dissociation in a shorter time, the inclusion of temperature plays the role of reducing the critical electric field necessary to this very same dissociation. Considering experimental data, a quantitative comparison with them is difficult to make since there is no information concerning how the field is distributed over microscopic scales [20] However, literature data reports a threshold value of around $10 \mathrm{mV} /$ Åin order to accomplish the exciton dissociation, in MEH-PPV systems Ref. [33], and $5 \mathrm{mV} /$ Åfor considering PPVs systems Ref. [34], which is in quite good agreement with the results of our simulation. Thus, thermal effects roughly halved the necessary electric field to separate a hole-polaron from an electron's. Adopting a phenomenological point of view, these results are expected, due to the fact that temperature induces the appearance of phonons that work towards the destabilization of the neutral quasi-particle.
Although interesting, the results previously discussed are somewhat idealized in the sense that no other topological defects, rather than the quasi-particles are present in the chain. It is well known that doping is a fundamental procedure in obtaining suitable materials for the organic electronic technology. The impurities act as providing an preferred region in which charge can accumulate to the formation of charge carriers, and also disturbs the dynamics of an already existing particle. In this sense, it is only natural to perform simulation with an impurity endowed chain and to compare these results to those of the pristine chain that have just been presented. The second Section 2 of Figure 4 reports the results of a doped chain with two $0.25 \mathrm{eV}$ impurities at $100 \mathrm{~K}$ and subjected to a $6.0 \mathrm{mV} /$ Åelectric field. The reason for using two impurities is the fact that the excitons perform a random walk dynamics, when temperature effects are taken into account. Thus, the exciton is not trapped by the fist impurity and, with in the presence of only one impurity, it is not possible observe the contribution of these effects on charge separation process. We observe that, even at this lower $(100 \mathrm{~K})$ temperature regime, the complete exciton dissociation is achieved at approximately the same time, when we can finally observe the separated charges. This is true, despite the transition phenomena that takes place from around $300 \mathrm{fs}$, when the splitting seems to begin to $900 \mathrm{fs}$ when it is completed. This transition consists on a mixed state between the coupled polarons and the desired condition of free charge. We shall return to this discussion shortly, when analyzing the same simulation from another point of view. Regardless of this difference on the charge separation mechanism, the final separation in the same total time of the previous case consists on a sort of trade-off between temperature and impurity interaction as far as dissociation time is concerned. As for the critical electric field, one can see that it is slightly increased in this case. Again, the effect of impurity partially accounts for the lower distortion provided by the thermal effects, thus overriding the low temperature considered.

The most interesting result, however, is obtained when we consider both the higher temperature regime of $300 \mathrm{~K}$ and the effect of the impurities. A strikingly difference is observed by a mere glimpse of the third Section 3 of Figure 4 . First of all, the dissociation times is shorter than half of those of the previous cases (1) and 

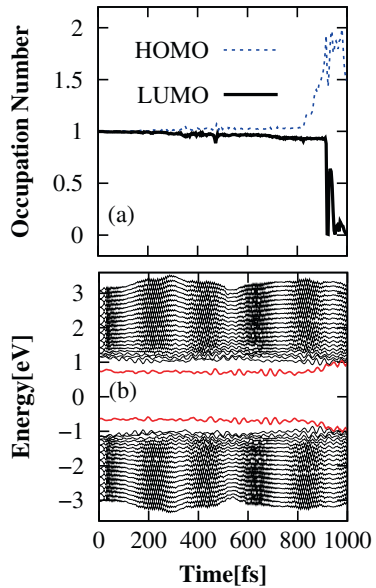

Fig. 5. Occupation numbers (a) and energy levels (b) time evolution for a pristine chain at $300 \mathrm{~K}$ subjected to an electric field of $5.0 \mathrm{mV} / \AA$.

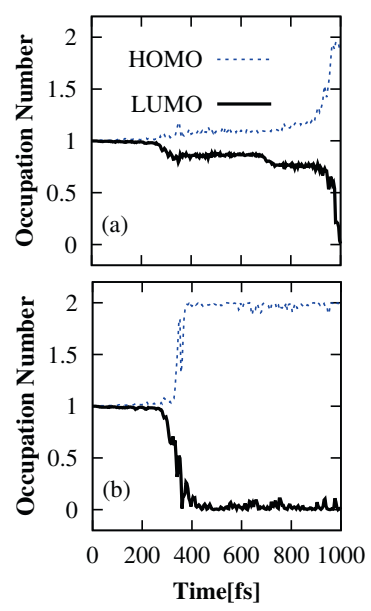

Fig. 6. Occupation numbers time evolution for the doped systems subjected to: (a) $100 \mathrm{~K}$ and $6.0 \mathrm{mV} / \mathrm{A}$ and (b) $300 \mathrm{~K}$ and $3.9 \mathrm{mV} / \AA . \AA$

(2) of Figure 4. While the temperature provides highly excited phonons that contributes to the separation of charge, the region of the placed impurity serves as a center of disturbance for the exciton, thus facilitating its dissociation into free charges. Another important feature is the decrease of the critical electric field to this dissociation. In this case, the interaction of thermal phonons together with the symmetry breaking impurities yielded as a critical field the value of $3.9 \mathrm{mV} / \AA$, which turned out to be the lowest ever reported for this kind of system [19,33,34]. We conclude that, when numerically describing the process of exciton dissociation in organic conductors, care must be taken in order to not underestimate the phenomena rate due to eventually not considering temperature and impurities effects. Our results suggest that the dissociation rate is expected to be higher than the previously predicted and can be further increased by thermalizing and by doping the real samples.

Another useful approach for discussing these results is the investigation of the occupation numbers. Concerning the last discussed point, namely the impurity effects, Figure 5 is very instructive in that it compares the occupation numbers - Figure $5(a)$ - with the energy levels time evolution Figure 5(b) for the pristine chain at $300 \mathrm{~K}$ and subjected to a $5.0 \mathrm{mV} /$ Åelectric field, i.e., the simulation presented in Figure 4(a1) and (b1). One can see that the returning of the excited electron to HOMO in Figure 5(a) coincides to the vanishing of the polaron levels in Figure 5(b) at about $900 \mathrm{fs}$. The inclusion of the energy levels profile is useful to confirm that this dynamics refers to the exciton dissociation, for after $900 \mathrm{fs}$ no polaron pair interaction is possible due to the destabilization of these quasi-particles. Obviously, the same pattern of time matching between the polarons levels reaching the conduction and valence band and the returning of the once populating LUMO electron to HOMO level is achieved for all the simulations, for these are merely two different ways of observing the same phenomena. Due to this fact, the energy levels time evolution profile are omitted from now on. We focus only in the occupation number analysis. Figure 6 presents the results for the occupation numbers for the doped systems simulations, previously presented in Figures 4(a2) and (a3). By investigating the upper part (a) of the figure, that regards the simulation at $100 \mathrm{~K}$ and $6.0 \mathrm{mV} / \AA \AA \AA$, the previously discussed same dissociation time as in Figure 5 is clearly noted. The most useful feature of this figure, however, is that it allow us to address the aforementioned transition structure (from exciton state to separated charges) to a partial charge transference from LUMO in the direction of HOMO, but without being completed until around $900 \mathrm{fs}$, when the dissociation is completely carried out. This comes from the partial splitting of the levels at around $300 \mathrm{fs}$ and the conclusion at around $900 \mathrm{fs}$ - the same 900 fs previously reported.

Finally, Figure 6(b) presents the case where the impurity acts together with the high thermal regime of $300 \mathrm{~K}$ in the sense of promoting the exciton dissociation in the shorter reported time. One can see that, in this case the dissociation takes place before the thermalization is accomplished. In other words, when both a high temperature and an impurity are present in the system, the dissociation occurs even before that particular thermal regime is stabilized. Additionally, one can conclude that the virtual lack of oscillation in the occupation levels is associated to a high dissociation yield and is partially due to the fact that, in this case, a lower critical electric field of $3.9 \mathrm{mV} /$ Åis necessary to accomplish the charge dissociation.

\section{Conclusions}

In summary, it was developed a modified version of the SSH model to include an external electric field, the Brazovskii-Kirova symmetry breaking term, impurities, and temperature, in order to investigate the effects of these properties over the dynamical process of singlet exciton dissociation in cis-symmetry conducting polymers chains. We were able to obtain at which temperature and electric field regimes the excitons lose their stability. The temperature effects reduce the critical electric field for the exciton dissociation to $5.0 \mathrm{mV} / \AA$. In the absence of this effect, the exciton is dissociated within an electric field regime of $7.9 \mathrm{mV} / \AA$, as reported in another theoretical work. Additionally, the presence of an impurity in the conjugated polymer lattice, favors the exciton dissociation mechanism. When both, impurity and temperature effects are taken into account, the electric field regime for the singlet exciton dissociation reduces to $3.9 \mathrm{mV} /$ Åat $300 \mathrm{~K}$. When only impurity effects are considered, the critical electric field is $5.7 \mathrm{mV} / \AA$, as investigate also in another theoretical study. In the low temperature regimes, the exciton is not trapped by the impurity and perform a random walk dynamics, a fact that is not observed in the absence of temperature. For a thermal regime of around $100 \mathrm{~K}$, in the presence of an impurity, the critical electric field for the exciton 
dissociation reduces to $6.0 \mathrm{mV} / \AA$. This is the minimal temperature in which the exciton dissociation occurs. The results indicate that when thermal effects are taken into account the singlet exciton dissociation dynamics in conjugated polymer systems is modified. This understanding can shine a light on the description of electroluminescence yields in PLDEs. Also, the discussion performed here on the behavior of the exciton dissociation is crucial for the field of organic conduction, thus providing guidance in the improvement of the charge carrier separation and transport efficiency in OSCs.

\section{Acknowledgements}

The authors gratefully acknowledge the financial support from the Brazilian Research Councils CNPq, CAPES and FINATEC.

\section{References}

[1] Y. Sun, G.C. Welch, W.L. Leong, C.J. Takacs, G.C. Bazan, A.J. Heeger, Nat. Mat. 11 (2012) 44

[2] D. Braga, N.C. Erickson, M.J. Renn, R.J. Holmes, C.D. Frisbie, Adv. Func. Mat. 22 (2012) 1623.

[3] L.P. Lu, D. Kabra, R.H. Friend, Adv. Func. Mat. 22 (2012) 4171

[4] Z. An, B. Di, C.Q. Wu, Wu. Eur. Phys. J. B. 63 (2008) 71.

[5] R.H. Friend, R.W. Gymer, A.B. Holmes, J.H. Burroughes, R.N. Marks, C. Taliani, D.D.C. Bradley, M. Lo, W.R. Salaneck, D.A. dos Santos, J.L. Brédas, Nature 397 (1999) 121.

[6] Y. Meng, B. Di, Y.D. Wang, X.J. Liu, Z. An, Eur. Phys. J. B. 85 (2012) 415.

[7] J.H. Burroughes, D.D.C. Bradley, A.R. Brown, R.N. Marks, K. Mackay, R.H. Friend, P. Burns, A B. Holmes, Nature 347 (1990) 539

[8] P.K.H. Ho, J.S. Kim, J.H. Burroughes, H. Becker, S.F.Y. Li, T.M. Brown, F. Cacialli, R.H. Friend, Nature 404 (2000) 481.
R.K.H.
[9] B. Kipplen, J.L. Brédas, Energy Environ. Sci. 251 (2009) 251.

[10] S.E. Shaheen, C.J. Brabec, N.S. Sariciftci, F. Padinger, T. Fromherz, J.C. Hummelen, Appl. Phys. Lett. 78 (2001) 841.

[11] D. Braun, A.J. Heeger, Appl. Phys. Lett. 58 (1991) 18

[12] A. Köhler, J.S. Wilson, R.H. Friend, Adv. Mater. 14 (2002) 791.

[12] A. Köhler, J.S. Wilson, R.H. Friend, Adv. Mater. 14 (2002)

[14] Q. Song, C. Li, M.C. Park, M. Lu, H. Yang, X. Hou. Phys. Rev. Lett. 98 (2207) 176403.

[15] Z. Shuai, D. Beljonne, R.J. Silbey, J.L. Brédas, Phys. Rev. Lett. 84 (2000) 131.

16] N.T. Harrison, G.R. Hayes, R.T. Phillips, R.H. Friend, Phys. Rev. Lett. 77 (1996) 1881

[17] L.A. Ribeiro, P.H. de Oliveira Neto, W.F. da Cunha, L.F. Roncaratti, R. Gargano, D.A. da Silva Filho, G.M. e Silva, J. Chem. Phys. 135 (2011) 224901

[18] P.H. de Oliveira Neto, W.F. da Cunha, L.F. Roncaratti, R. Gargano, G.M. e Silva, Chem. Phys. Lett. 493 (2010) 283.

[19] Z. Hong-Xia, Z. Hui, C. Yu-Guang, Chin. Phys. Lett. 28 (2011) 097201; Z. Hong-Xia, Z Hui, C. Yu-Guang, Chin. Phys. Lett. 116 (2001) 57.

[20] S. Stafström, J. Lumin. 112 (2005) 357.

[21] A.J. Heeger, S. Kivelson, J.R. Schrieffer, W.P. Su, Rev. Mod. Phys. 60 (1988) 781

[22] W.P. Su, J.R. Schrieffer, A.J. Heeger, Phys. Rev. B 22 (1980) 2099.

23] A.J. Hegger, Angew. Chem. Int. 40 (2001) 2591.

[24] A.J. Hegger, Rev. Mod. Phys. 73 (2001) 681.

[25] L.A. Ribeiro, P.H. de Oliveira Neto, W.F. da Cunha, G.M. e Silva, Phys. Proc. 28 (2012) 112 .

[26] L.A. Ribeiro, P.H. de Oliveira Neto, W.F. da Cunha, R. Gargano, G.M. e Silva, Chem. Phys. Lett. 555 (2013) 168.

27] G.M. e Silva, Phys. Rev. B 61 (2000) 10777.

28] Y. Ono, A. Terai, J. Phys. Soc. Jpn. 59 (1990) 2893.

29] D. Roy, Phys. Rev. E 77 (2008) 062102.

[30] D. Banerjee, B.C. Bag, S.K. Banika, D.S. Rayb, J. Chem. Phys. 120 (2004) 8960.

[31] L.F. Roncaratti, R. Gargano, G.M. e Silva, J. Phys. Chem. A. 113 (2009) 14591.

[32] G. Cerullo, G. Lanzani, M.Z.S.S. de Silvestri, S.D. Comoretto, I. Moggio, G. Dellepiane, Synth. Met. 116 (2001) 57.

[33] M.I. Khan, G.C. Bazan, Z.D. Popovic, Chem. Phys. Lett. 298 (1998) 309.

[34] R. Kersting, U. Lemmer, M. Deussen, H.J. Bakker, R.F. Mahrt, H. Kurz, V.I Arkhipov, H. Bassler, Phys. Rev. Lett. 73 (1994) 1440 


\title{
APPENDIX \\ PAPER V
}

\section{Impurity Effects on Polaron-Exciton Formation in Conjugated Polymers}

\author{
Luiz Antonio Ribeiro Junior \\ Wiliam Ferreira da Cunha \\ Pedro Henrique de Oliveira Neto \\ Ricardo Gargano \\ Geraldo Magela e Silva
}

J. Chem. Phys., 189, 174903-6 (2013) 


\title{
Impurity effects on polaron-exciton formation in conjugated polymers
}

\author{
Luiz Antonio Ribeiro, Jr., ${ }^{\text {a) }}$ Wiliam Ferreira da Cunha, Pedro Henrique de Oliveira Neto, \\ Ricardo Gargano, and Geraldo Magela e Silva \\ Institute of Physics, University of Brasilia, 70.919-970 Brasilia, Brazil
}

(Received 18 July 2013; accepted 19 October 2013; published online 5 November 2013)

\begin{abstract}
Combining the one-dimensional tight-binding Su-Schrieffer-Heeger model and the extended Hubbard model, the collision of two oppositely charged polarons is investigated under the influence of impurity effects using a non-adiabatic evolution method. Results show that electron-electron interactions have direct influence on the charge distribution coupled to the polaron-exciton lattice defect. Additionally, the presence of an impurity in the collisional process reduces the critical electric field for the polaron-exciton formation. In the small electric field regime, the impurity effects open three channels and are of fundamental importance to favor the polaron-exciton creation. The results indicate that the scattering between polarons in the presence of impurities can throw a new light on the description of electroluminescence in conjugated polymer systems. (C) 2013 AIP Publishing LLC. [http://dx.doi.org/10.1063/1.4828726]
\end{abstract}

\section{INTRODUCTION}

Conjugated polymers have attracted considerable interest from the scientific community since the discovery of electroluminescence properties on phenyl-based organic semiconductors. Their optoelectronic features combined with ease of synthesis, low temperature processing, tunability via synthetic chemistry, and low cost, make them attractive materials for the electronics industry, particularly concerning the development of a new display technology. Among the several possible application are Organic Photovoltaics devices (OPVs) $)^{1-3}$ and Polymer Light Emitting Diodes (PLEDs). ${ }^{4-6}$ In these devices, the generation of excited states is one of the most fundamental physical process. ${ }^{7-11}$ Lei et al. ${ }^{12}$ studied the dependence of exciton formation rate on the spin orientation of polarons by simulating the collision process of two oppositely charged polarons, using a modified version of $\mathrm{Su}-$ Schrieffer-Heeger (SSH) model. The role played by the spin configuration in the collision dynamics of these charge carriers is a question of major importance for electroluminescence process in PLEDs. Their results show that the yield of singlet excitons from parallel spin or antiparallel spin polarons configuration is not influenced by the $e-e$ interactions. On the other hand, in the manufacture of spin PLEDs to improve the luminescent efficiency, the $e-e$ interactions should be critical factors, whose role must be clarified. Particularly, understanding how internal effects such as $e-e$ interactions and impurity effects can favor or unfavor the polaron-exciton formation via scattering of oppositely charged polarons is an issue that is believed to be crucial for the design of more efficient devices with respect to the electroluminescence. Thus, this point requires a better phenomenological description.

Some relevant theoretical studies carried out by An and co-workers ${ }^{13}$ have shown that external electric field has a significant influence on polaron-exciton formation via

$\overline{\text { a) Electronic mail: ribeirojr@ fis.unb.br }}$ polaron-pair scattering in conjugated polymers. The goal was to identify the generation mechanism of the self-trapped polaron-exciton. Their results show that, for the scattering processes of the two polarons initially presented on a same polymer chain, three regimes of the applied electric field strength were identified: (1) at field strength smaller than $0.2 \mathrm{mV} / \AA$, the polaron-pair scatter into an exciton state after $1300 \mathrm{fs}$; (2) for an electric field strength between $0.2 \mathrm{mV} / \AA$ and $1.2 \mathrm{mV} / \AA$, the polaron-pair scatters into a pair of independent particles and each of them is a mix of polarons and excitons (in this case, the yield of the neutral exciton depends sensitively on the electric field strength); and (3) at electric field strength greater than $1.2 \mathrm{mV} / \AA$, the two polarons break into irregular lattice vibrations after their collision. When two polymer chains are taken into account, the two polarons will combine together to form a self-trapped exciton in one of the two coupled polymer chains when they lie initially on the different polymer chains. The results indicate that the interchain interaction favors the formation of the polaron-exciton. It is important to remark that these studies have been focused on specific cases with idealized conditions. A theory that widely holds true for real materials needs further verifying by addressing some realistic effects, for example, the order degree or molecules, temperature and impurity effects. Also, from these works, we can see that all the results for the polaron-pair interaction are not fully described, so that further investigations that take into account some of these effects are needed.

In this paper, a systematic numerical investigation of polaron-exciton formation was performed in a cispolyacetylene chain in terms of a non-adiabatic evolution method. The collision of oppositely charged polarons was investigated on a conjugated polymer chain subjected to different field strengths, one-site, and nearest-neighbor Coulomb interactions. We carried out the molecular dynamics by using an one-dimensional tight-binding model including lattice relaxation. Combined with the extended Hubbard model 
(EHM), an extended version of the SSH model was used to include external electric fields and Brazoviskii-Kirova symmetry breaking term. The aim of this paper is to give a physical picture of the polaron-exciton formation in conjugated polymers, when the $e-e$ interactions and impurity effects are taken into account, and contribute to the understanding of these important processes that may provide guidance for improving the electroluminescence efficiency in PLEDs.

\section{METHODOLOGY}

A polyacetylene chain in cis configuration ${ }^{14}$ was used to study collision between oppositely charged polarons under the influence of impurity effects in conjugated polymers. The overall Hamiltonian is given by

$$
H_{\text {total }}=H_{S S H}+H_{e e}+H_{i m p} .
$$

The first term in Eq. (1) is the SSH-type Hamiltonian modified to include an external electric field and the BrazovskiiKirova symmetry-breaking, which has the following form:

$$
\begin{aligned}
H_{S S H}= & -\sum_{n, s}\left(t_{n, n+1} C_{n+1, s}^{\dagger} C_{n, s}+\text { h.c. }\right) \\
& +\sum_{n} \frac{K}{2} y_{n}^{2}+\sum_{n} \frac{p_{n}^{2}}{2 M},
\end{aligned}
$$

where $n$ indexes the sites of the chain. The operator $C_{n, s}^{\dagger}\left(C_{n, s}\right)$ creates (annihilates) a $\pi$-electron state at the $n$th site with spin $s ; K$ is the harmonic constant that describes a $\sigma$ bond and $M$ is the mass of a $\mathrm{CH}$ group. The parameter $y_{n}$ is defined as $y_{n} \equiv u_{n+1}-u_{n}$ where $u_{n}$ is the lattice displacement of an atom at the $n$th site. $p_{n}$ is the momentum conjugated to $u_{n}$ and $t_{n, n+1}$ is the hopping integral, given by

$$
t_{n, n+1}=e^{-i \gamma \mathbf{A} t}\left[\left(1+(-1)^{n} \delta_{0}\right) t_{0}-\alpha y_{n}\right],
$$

where $t_{0}$ is the hopping integral of a $\pi$-electron between nearest-neighbor sites in the undimerized chain, $\alpha$ is the electron-phonon coupling, and $\delta_{0}$ is the Brazovskii-Kirova symmetry-breaking term, which is used to take the cis symmetry of the polymer into account. $\gamma \equiv e a /(\hbar c)$, with $e$ being the absolute value of the electronic charge, $a$ is the lattice constant, and $c$ is the speed of light. The relation between the time-dependent vector potential $\mathbf{A}$ and the uniform electric field $\mathbf{E}$ is given by $\mathbf{E}=-(1 / c) \dot{\mathbf{A}}$.

The second contribution in Eq. (1) denotes $e$ - $e$ interactions and can be written as

$$
\begin{aligned}
H_{e e}= & U \sum_{i}\left(C_{i, \uparrow}^{\dagger} C_{i, \uparrow}-\frac{1}{2}\right)\left(C_{i, \downarrow}^{\dagger} C_{i, \downarrow}-\frac{1}{2}\right) \\
& +V \sum_{i}\left(n_{i}-1\right)\left(n_{i+1}-1\right),
\end{aligned}
$$

where $U$ and $V$ are the on-site and nearest-neighbor Coulomb repulsion strengths, respectively, and $n_{i}=C_{i, \uparrow}^{\dagger} C_{i, \uparrow}+C_{i, \downarrow}^{\dagger} C_{i, \downarrow}$. It should be noted that the inclusion of the additional constant factors (related to the conventional description of the Hubbard model) is necessary in order to maintain the electron hole symmetry of the Hamiltonian.
The last contribution in Eq. (1) represents the on-site impurity effects and can be written in the form

$$
H_{i m p}=Z_{j} C_{j, s}^{\dagger} C_{j, s} \text {. }
$$

$Z_{j}$ is the strength of an impurity, which is located in $j$ th site. The parameters used here are $t_{0}=2.5 \mathrm{eV}$, $M=1349.14 \mathrm{eV} \times \mathrm{fs}^{2} / \AA^{2}, K=21 \mathrm{eV}^{-2}, \quad \delta_{0}=0.05$, $\alpha=4.1 \mathrm{eV} \AA^{-1}, a=1.22 \AA$, and a bare optical phonon energy $\hbar \omega_{Q}=\hbar \sqrt{4 K / M}=0.16 \mathrm{eV}$. These values have been used in previous simulations and are expected to be valid for conjugated polymers in general. ${ }^{15-19}$

In order to solve these equations numerically, first a stationary state that is self-consistent with all degrees of freedom of the system (the lattice plus electrons) is obtained. We begin by constructing the Hamiltonian from an arbitrary $\left\{\mathrm{y}_{n}\right\}$ set of positions. By solving the time dependent Schrödinger equation, a new set of coordinates $\left\{\mathrm{y}_{n}{ }_{n}\right\}$ is obtained. Iterative repetitions of this procedure yields a self-consistent initial state when $\left\{\mathrm{y}_{n}{ }_{n}\right\}$ is close enough to $\left\{\mathrm{y}_{n}\right\}$.

The time evolution of the system is described by the equations of motion. The electronic wave function is the solution of the time-dependent Schrödinger equation

$$
\begin{aligned}
i \hbar \dot{\psi}_{k, s}(i, t)= & -\left[t_{i, i+1}^{*}+V \tau_{s}(i, t)\right] \psi_{k, s}(i+1, t) \\
& -\left[t_{i-1}, i+V \tau_{s}^{*}(i-1, t)\right] \psi_{k, s}(i-1, t) \\
& +\left\{U\left[\rho_{-s}(i, t)-\frac{1}{2}\right]+Z_{j}\right. \\
& \left.+V \sum_{s^{\prime}}\left[\rho_{s^{\prime}}(i+1, t)+\rho_{s^{\prime}}(i-1, t)-1\right]\right\} \\
& \times \psi_{k, s}(i, t),
\end{aligned}
$$

where $k$ is the quantum number that specifies an electronic state,

$$
\rho_{s}(i, t)=\sum_{k}{ }^{\prime} \psi_{k, s}^{*}(i, t) \psi_{k, s}(i, t),
$$

and

$$
\tau_{s}(i, t)=\sum_{k}{ }^{\prime} \psi_{k, s}^{*}(i+1, t) \psi_{k, s}(i, t) .
$$

The prime symbol specifies that the sum runs over occupied single-particle states. It is important to note that, considering the hydrocarbon nature of our system, an unrestricted Harteee Fock scheme is sufficient to correctly address the desired charge transport properties.

The equation of motion that describes the site displacement and provides the temporal evolution of the lattice is obtained by a classical approach. ${ }^{14}$ The nuclear dynamics is carried out by considering the Euler-Lagrange equations

$$
\frac{d}{d t}\left(\frac{\partial\langle L\rangle}{\partial \dot{u}_{n}}\right)-\frac{\partial\langle L\rangle}{\partial u_{n}}=0,
$$

where

$$
\langle L\rangle=\langle T\rangle-\langle V\rangle .
$$

Equation (9) leads to

$$
M \ddot{u}_{n}=F_{n}(t),
$$


where

$$
\begin{aligned}
F_{n}(t)= & -K\left[2 u_{n}(t)-u_{n+1}(t)-u_{n-1}(t)\right] \\
& +\alpha\left[B_{n, n+1}-B_{n-1, n}+B_{n+1, n}-B_{n, n-1}\right] .
\end{aligned}
$$

$F_{n}(t)$ represents the force on the $n$th site. Here,

$$
B_{n, n^{\prime}}=\sum_{k, s}{ }^{\prime} \psi_{k, s}^{*}(n, t) \psi_{k, s}\left(n^{\prime}, t\right)
$$

is the term that couples the electronic and lattice solutions.

The total time dependent wave function is constructed by means of a combination of instantaneous eigenstates of the electronic Hamiltonian. The solutions of the time-dependent Schrödinger equation can be put in the form ${ }^{20}$

$$
\begin{aligned}
\psi_{k, s}\left(n, t_{j+1}\right)= & \sum_{l}\left[\sum_{m} \phi_{l, s}^{*}\left(m, t_{j}\right) \psi_{k, s}\left(m, t_{j}\right)\right] \\
& \times e^{\left(-i \varepsilon_{l} \Delta t / \hbar\right)} \phi_{l, s}\left(n, t_{j}\right) .
\end{aligned}
$$

$\left\{\phi_{l}(n)\right\}$ and $\left\{\varepsilon_{l}\right\}$ are the eigenfunctions and the eigenvalues of the electronic part for the hamiltonian at a given time $t_{j}$. Equation (12), which governs the evolution of the lattice, may be numerically integrated using the following method: ${ }^{21}$

$$
\begin{aligned}
& u_{n}\left(t_{j+1}\right)=u_{n}\left(t_{j}\right)+\dot{u}_{n}\left(t_{j}\right) \Delta t, \\
& \dot{u}_{n}\left(t_{j+1}\right)=\dot{u}_{n}\left(t_{j}\right)+\frac{F_{n}\left(t_{j}\right)}{M} \Delta t .
\end{aligned}
$$

Hence, the electronic wave functions and the lattice displacements at the $(j+1)$ th time step are obtained from the $j$ th time step. At time $t_{j}$, the wave functions $\left\{\psi_{k, s}\left(i, t_{j}\right)\right\}$ can be expressed as a series expansion of the eigenfunctions $\left\{\phi_{l, s}\right\}$ at that moment

$$
\psi_{k, s}\left(i, t_{j}\right)=\sum_{l=1}^{N} C_{l, k}^{s} \phi_{l, s}(i),
$$

where $C_{l, k}^{s}$ are the expansion coefficients. The occupation number for eigenstate $\phi_{l, s}$ is

$$
\eta_{l, s}\left(t_{j}\right)=\sum_{k}{ }^{\prime}\left|C_{l, k}^{s}\left(t_{j}\right)\right|^{2} .
$$

$\eta_{l, s}\left(t_{j}\right)$ contains information concerning the redistribution of electrons among the energy levels..$^{22,23}$

\section{RESULTS AND DISCUSSION}

In this work, we performed a series of simulations concerning collisional processes between oppositely charged polarons in a 200 -site cis-polyacetylene chain. In order to mitigate end effects of the chain end, periodic boundary conditions were imposed to our systems. This way, we simulate a chain of infinite length, thus reducing effects of numerical nature, due to the symmetry breaking imposed by the edge. The aim was to investigate the effects of impurities, electric field, and Coulomb interactions over the consequent formation of polaron-exciton after the interaction of the polaron-pair. For the electric field, the values used in the simulations varied from 0.1 to $1.0 \mathrm{mV} /$ Åwith a increment of $0.1 \mathrm{mV} / \AA$, whereas the on-site $e$-e interactions values considered are $0.1,0.2,0.3,0.4$, and $0.5 \mathrm{eV}$. The nearestneighbor Coulomb repulsion strength was defined using the relation $V=U / 2$. In the present work, we considered the impurity in the 100th site and created oppositely charged polarons initially at sites 50 and 150 . It is important to remark that this procedure is carried out without loss of generality, because the impurities act like a long range coulombic potential that affects all its neighborhood. Our analysis is carried out by considering a mean charge density defined by $\bar{\rho}(t)=1-\left[\rho_{n-1}(t)+2 \rho_{n}(t)+\rho_{n+1}(t)\right] / 4$ and a mean order parameter of the lattice distortion $\bar{y}(t)=(-1)^{n}\left[y_{n-1}(t)-2 y_{n}(t)+y_{n+1}(t)\right] / 4$. The goal is to provide a better visualization of the simulations and consequently to perform a more accurate analysis of the results. We should emphasize that, although spins effects are known to be of fundamental importance for the recombination process of polarons, as described in the literature, ${ }^{23}$ our goal is to investigate effects that are not spin dependent. ${ }^{12}$

In this context, Figure 1(a) shows the schematic diagram of energy-levels for a polymer chain containing an oppositely charged polaron-pair with antiparallel spin configuration. These states are constructed by occupying the different energy levels, which correspond to consider the sum in Eq. (13) over different states. There are four levels in the gap. The two left levels, $\varepsilon_{L}^{1}$ and $\varepsilon_{L}^{2}$, represent the electronic configuration for an electron-polaron, in which the upper one is occupied by one electron and the lower one occupied by two. The right levels, $\varepsilon_{R}^{1}$ and $\varepsilon_{R}^{2}$, represent the electronic configuration for a hole-polaron, where only the $\varepsilon_{R}^{1}$ level is occupied by one electron. Figure 1(b) represents an alternative route to creating a oppositely charged polaron-pair, with parallel spin configuration. It is well known that the exciton formation rate depends on the spin orientation of polarons. Also, it is accepted that the yield of singlet excitons from parallel spin or antiparallel spin configuration of a polaron-pair is not influenced by the $e$-e interactions. ${ }^{12}$ A first important result that we can remark is that, indeed, no qualitative difference is found on the dynamical behavior of a polaron-pair created through mechanism of Figure 1(a) or 1(b), when impurity effects are taken into account. We thus present results solely concerning the band structure configuration shown in Figure 1(a) throughout this paper.

We begin by discussing the dynamic features of the collisional process between oppositely charged polarons considering the contribution of impurity effects to this mechanism. Figure 2 presents a set of simulations for the chain endowed with a $0.2 \mathrm{eV}$ impurity in the 100th site. We

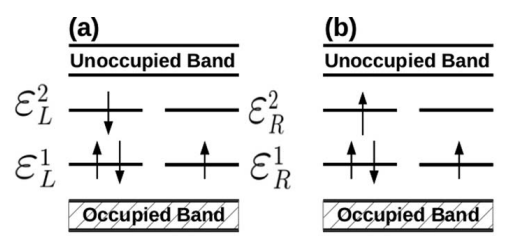

FIG. 1. The schematic diagrams of energy levels for a polymer chain containing a electron-polaron and a hole-polaron with (a) antiparallel spin and (b) parallel spin. 
174903-4 Ribeiro, Jr. et al.

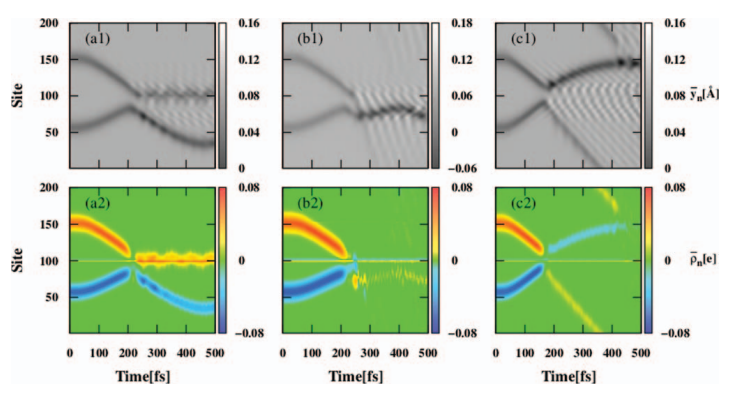

FIG. 2. Collision between oppositely charged polarons in a polymer chain in the presence of an impurity: (a1) and (a2) $E=0.2 \mathrm{mV} / \AA$ and $U=0.1 \mathrm{eV}$ (b1) and (b2) $E=0.2 \mathrm{mV} / \AA ̊$ and $U=0.2 \mathrm{eV}$; (c1) and (c2) $E=0.4 \mathrm{mV} / \AA$ and $U=0.2 \mathrm{eV}$.

choose this value because it is the typical value observed for the potential barrier of PLEDs and for means of comparison, since it has recently been used in other theoretical work. ${ }^{24}$ In this case, one can observe a difference on the lattice distortion between different Hubbard terms, a feature that is represented on the upper part of Figure 2 (Figures 2(a1), 2(b1), and 2(c1)). Figures 2(a1)-2(b2) represent the simulation of an electric field of $0.2 \mathrm{mV} /$ A and Hubbard terms of 0.1 and $0.2 \mathrm{eV}$, respectively, whereas Figures 2(c1) and 2(c2) show the result of the application of a $0.4 \mathrm{mV} /$ Afor the electric field and a Hubbard term of $0.2 \mathrm{eV}$. These values were chosen due to the fact that they guarantee the stability of the polaron-exciton

throughout the whole simulation. After a small transient time for the electric field response, the polarons begin to move towards one another due to the opposite charges. The polaron dynamics before the collision processes occurs as described by Stafström and Johansson. ${ }^{25,26}$ At approximately $200 \mathrm{fs}$, when the collision takes place, one can see that the positive polaron passes through the negative polaron, a process that yields the formation of a mixed state of polaron and exciton after the collision - thus the name "polaron-exciton." We note that the polaron-exciton emerging from the collision is created at approximately the same time. As a matter of fact, we noted an even more general pattern: for a pristine chain and electric fields smaller than $0.2 \mathrm{mV} / \AA$, no polaron-exciton creation is seen to take place before $1500 \mathrm{fs},{ }^{13}$ whereas when the impurity is considered, the creation time falls to at most $400 \mathrm{fs}$. This is the first evidence that, in these systems, impurity acts as an exciton creating enabler. Another important effect that impurity has over the systems dynamics is readily noted by observing Fig. 2. When impurity effects are taken into account, three formation channels are observed: (1) After the collision one of the polarons becomes trapped by the potential of the impurity. This feature can be observed both in the lattice distortion Figure 2(a1) and in the charge density Figure 2(a2). An interesting observation not presented by the figure is that this pattern is observed to take place for $U=0.1 \mathrm{eV}$ regardless the electric field considered. (2) Figure 2(b) is the situation in which we only increased the Hubbard interaction to $0.2 \mathrm{eV}$. One can see that after the
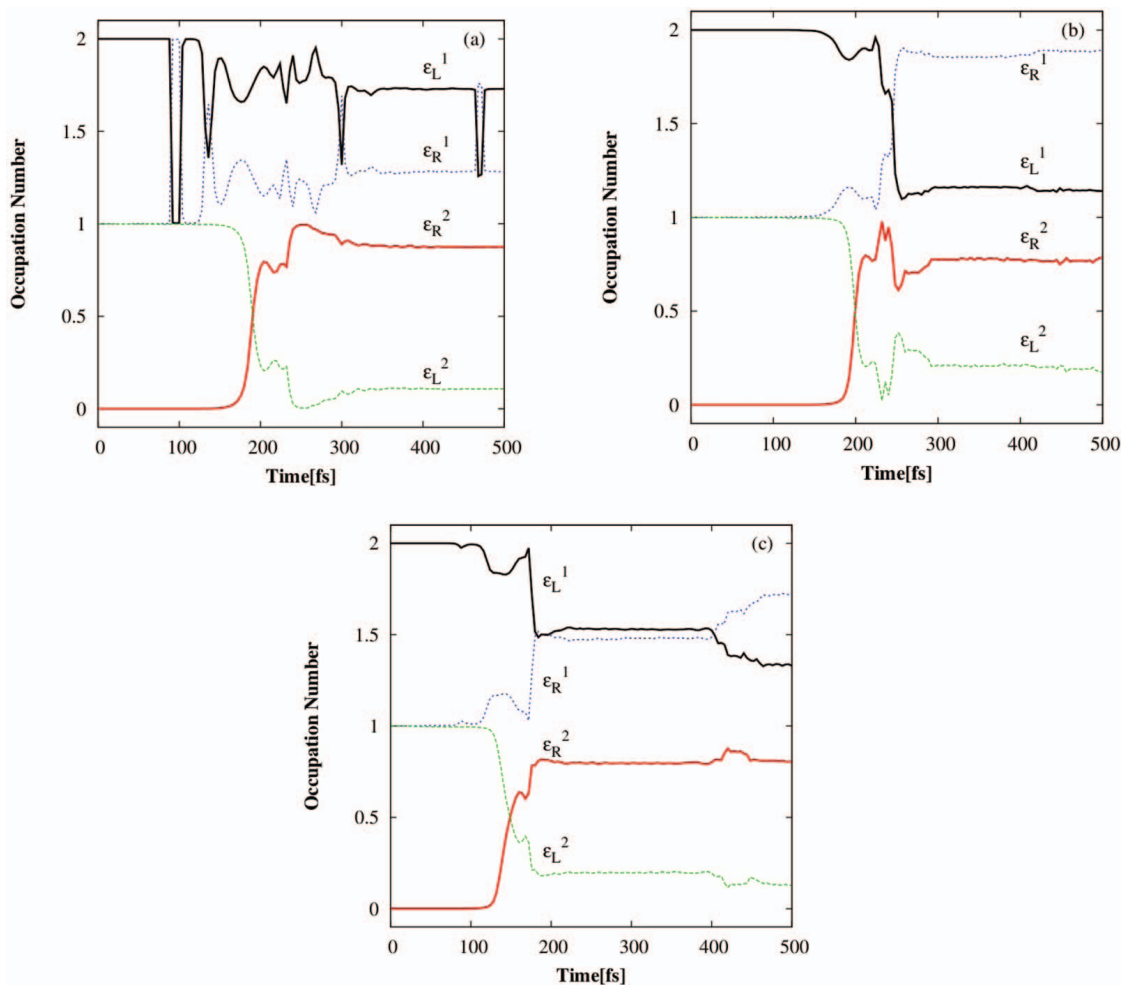

FIG. 3. Time evolution of the occupation number for the cases shown in Figure 2. 
collision one of the polarons ceases to be present whereas the charge tends to be a little more delocalized over the chain. (3) Finally, Figure 2(c) represents the previous case with an increase on the electric field, as can be inferred by the greater slope on the order parameter of Figure 2(c1). In this case, we can see that no polaron becomes trapped by the impurity nor is destroyed in the collision process.

The previous discussion on the creation of polaronexcitons is confirmed by an analysis of the occupation numbers presented in Figure 3. Note that this figure is not to be confused with the schematic representation of Figure 1. Whereas Figure 1 presents the static energy levels distributions for the chains containing different charge carriers in the initial state, Figure 3 represents the time evolution of the occupation numbers of the levels related to Figure 1(a). This figure represents the whole system: $\varepsilon_{L}^{1}$ and $\varepsilon_{L}^{2}$, stand for the states of the negatively charged polaron while $\varepsilon_{R}^{1}$ and $\varepsilon_{R}^{2}$, represent the states of the positive one. After the transient period in which the occupation number also oscillates due to the collision, we note a considerable degree of symmetry between $\varepsilon_{L}^{1}$ and $\varepsilon_{R}^{1}$ and also between $\varepsilon_{L}^{2}$ and $\varepsilon_{R}^{2}$, which is an indication of an electron exchange between these levels. The crossed and horizontal electron exchange between the levels represents the formation of a polaron-exciton structure. By analyzing the occupation number itself, one can see that the final states are not covered by integer numbers, which is an evidence of partial transfer. Even so, our simulations yield excitations with a better rate than those previously reported in the work by An. ${ }^{13}$ We believe that the degeneracy breaking provided by the inclusion of Hubbard terms, absent in the work of An, ${ }^{13}$ is responsible for this better yield of excitation. In the absence of an impurity in the lattice, studies reported that there exists only one channel for the polaron-exciton formation: the scattering of the polaron-pair after the interaction. ${ }^{12,13}$ However, when impurity effects are taken into account, the polaronexciton formation occurs even in the collision of the polaronpair. This fact can be noted observing Figures 3(a) and 3(b), where the time evolution of the occupation numbers indicates an electron exchange between the levels that represent the formation of a polaron-exciton structure.

While the occupation number analysis is the most suitable tool in studying the process of polaron-exciton creation, the stability of the quasi-particle is better described by means of the energy levels time evolution. We finish our discussion by presenting in Figure 4 the energy levels time evolution profile of the simulations correspondent to an interaction between polarons shown in Figure 2. The collisional process is noted to take place at approximately $200 \mathrm{fs}$, when the resulting phonons are represented by the oscillation of the energy levels. The signature of the loss of stability of one of the polarons is related to the red states returning to the conducting and valence bands. As in Figures 4(b) and 4(c), we see that the other polaron remains stable through the rest of the simulation, since the blue polarons levels remain
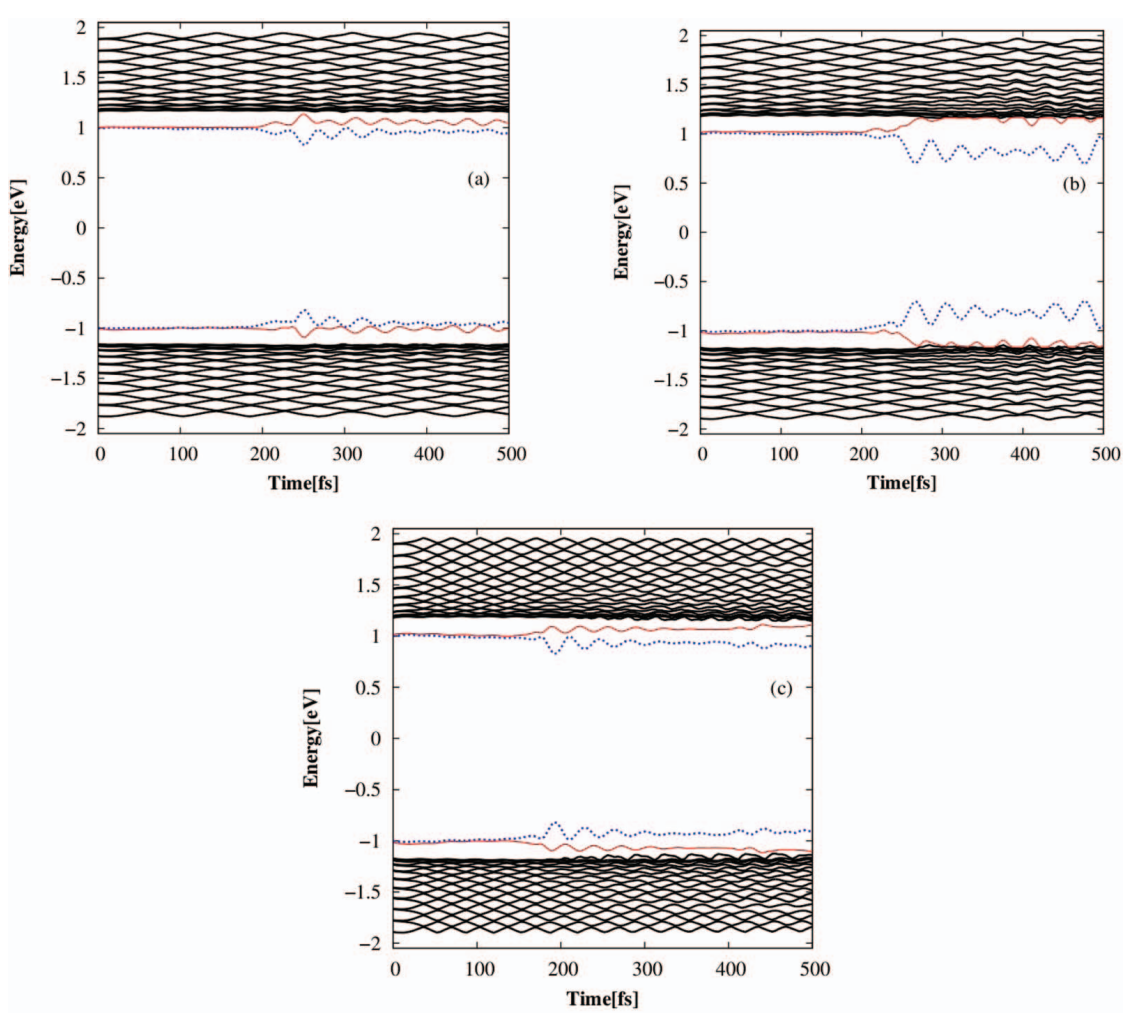

FIG. 4. Time evolution of the energy levels for the cases shown in Figure 2. 
consistently inside the bandgap. In Figures 2(a1) and 2(a2), both polaron-excitons keep the integrity until the end of simulation. This can be seen in Figure 4(a), where the energy levels that correspond to the polarons remain inside the bandgap.

\section{CONCLUSIONS}

In summary, it was presented a modified version of the SSH model to include an external electric field, the Brazovskii-Kirova symmetry breaking term, one-site and nearest-neighbor Coulomb interactions, and impurities in order to investigate the effects of these properties over the scattering process of oppositely charged polarons in cis-symmetry conducting polymers chains. Using a non-adiabatic evolution method, within an one-dimensional tight-binding model, the critical electric field regimes of polaron-exciton formation is described. The influence of $e-e$ interactions on the polaronexciton formation is also discussed and it is found to play an important role on the yield of excitations after the polaronpair scattering process. The charge coupled to the polaronexciton lattice deformation is sensitive to on-site and nearestneighbor Coulomb interaction. The higher the Coulomb interaction values the less the charge remains coupled to the polaron-exciton lattice deformation and the excitation yield is improved. When the impurity effects are taken into account, the critical electric field to polaron-exciton formation is lower than in the absence of these effects. Also, the results indicate that the presence of impurities in a conjugated polymer chain, for all electric fields regimes, improves the excitation yield and facilitates the polaron-exciton formation in electric field regimes smaller than $0.7 \mathrm{mV} / \AA$. These properties may provide guidance for improving the electroluminescence in polymer light-emitting diodes by defining a path considering impurities and materials with greater electronic correlation.

\section{ACKNOWLEDGMENTS}

The authors gratefully acknowledge the financial support from the Brazilian Research Councils CNPq, CAPES, and FINATEC.
${ }^{1}$ S. M. Menke, W. A. Luhman, and R. J. Holmes, Nature Mater. 12, 152-157 (2013)

${ }^{2}$ A. E. Jailaubekov, A. P. Willard, J. R. Tritsch, W.-L. Chan, N. Sai, R. Gearba, L. G. Kaake, K. J. Williams, K. Leung, P. J. Rossky, and X.-Y. Zhu, Nature Mater. 12, 66-73 (2013).

${ }^{3}$ G. Grancini, M. Maiuri, D. Fazzi, A. Petrozza, H.-J. Egelhaaf, D. Brida, G. Cerullo, and G. Lanzani, Nature Mater. 12, 29-33 (2013).

${ }^{4}$ Y. Qing, L. Ying, P. Caofeng, J. Chen, X. Wen, and Z. L. Wang, Nano Lett. 13, 607-613 (2013).

${ }^{5}$ S. Tang, J. Pan, H. A. Buchholz, and L. Edman, J. Am. Chem. Soc. 135, 3647-3652 (2013).

${ }^{6}$ C. A. Zuniga, J. Abdallah, W. Haske, Y. Zhang, V. Coropceanu, S. Barlow, B. Kippelen, and S. R. Marder, Adv. Mater. 25, 1739-1744 (2013).

${ }^{7}$ W. Barford, J. Phys. Chem. A 117, 2665-2671 (2013).

${ }^{8}$ W. Barford, Phys. Rev. B 70, 205204-205212 (2004).

${ }^{9}$ R. H. Friend, R. W. Gymer, A. B. Holmes, J. H. Burroughes, R. N. Marks, C. Taliani, D. C. C. Bradley, M. Lo, W. R. Salaneck, D. A. dos Santos, and J.-L. Brdas, Nature (London) 397, 121-128 (1999).

${ }^{10}$ J. H. Burroughes, D. D. C. Bradley, A. R. Brown, R. N. Marks, K. Mackay, R. H. Friend, P. L. Burns, and A. B. Holmes, Nature (London) 347, 539 (1990).

${ }^{11}$ P. K. H. Ho, J. S. Kim, J. H. Burroughes, H. Becker, S. F. Y. Li, T M. Brown, F. Cacialli, and R. H. Friend, Nature (London) 404, 481 (2000).

12 J. Lei, Z. Sun, Y. Zhang, and S. Xie, Org. Electron. 10, 1489 (2009).

${ }^{13}$ Z. An, B. Di, and C. Q. Wu, Eur. Phys. J. B 63, 71 (2008).

${ }^{14}$ A. J. Heeger, Rev. Mod. Phys. 73, 681 (2001)

${ }^{15}$ L. A. Ribeiro, P. H. de Oliveira Neto, W. F. da Cunha, L. F. Roncaratti, R. Gargano, D. A. da Silva Filho, and G. M. e Silva, J. Chem. Phys. 135, 224901 (2011).

${ }^{16}$ L. A. Ribeiro, P. H. de Oliveira Neto, W. F. da Cunha, R. Gargano, and G. M. e Silva, Chem. Phys. Lett. 555, 168 (2013).

${ }^{17}$ L. A. Ribeiro, P. H. de Oliveira Neto, W. F. da Cunha, R. Gargano, and G. M. e Silva, Chem. Phys. Lett. 580, 108-114 (2013).

${ }^{18}$ L. A. Ribeiro, P. H. de Oliveira Neto, W. F. da Cunha, and G. M. e Silva, Phys. Proc. 28, 112 (2012)

${ }^{19}$ L. A. Ribeiro, P. H. de Oliveira Neto, W. F. da Cunha, R. Gargano, and G. M. e Silva, J. Chem. Phys. B. 117, 11801-11811 (2013).

${ }^{20}$ M. P. Lima and G. M. e Silva, Phys. Rev. B 74, 224304 (2006).

${ }^{21}$ G. M. e Silva, Phys. Rev. B 61, 10777 (2000).

${ }^{22}$ Z. Sun, D. S. Liu, S. Stafström, and Z. An, J. Chem. Phys. 134, 044906 (2011).

${ }^{23}$ Z. Sun and S. Stafström, J. Chem. Phys. 136, 244901 (2012).

${ }^{24}$ B. Di, Y. Meng, Y. D. Wang, X. J. Liu, and Z. An, J. Phys. Chem. B 115, 964 (2011).

${ }^{25}$ S. Stafström, Chem. Soc. Rev. 39, 2484 (2010).

26 A. Johansson and S. Stafström, Phys. Rev. Lett. 86, 3602 (2001). 


\section{APPENDIX PAPER VI}

\section{Dynamical Study of Impurity Effects on Bipolaron-Bipolaron and Bipolaron-Polaron Scattering in Conjugated Polymers}

Luiz Antonio Ribeiro Junior Wiliam Ferreira da Cunha Pedro Henrique de Oliveira Neto Ricardo Gargano Geraldo Magela e Silva

J. Phys. Chem. B., 117, 11801-11811 (2013) 


\section{Dynamical Study of Impurity Effects on Bipolaron-Bipolaron and Bipolaron-Polaron Scattering in Conjugated Polymers}

Luiz Antonio Ribeiro, Wiliam Ferreira da Cunha, Pedro Henrique de Oliveira Neto, Ricardo Gargano, and Geraldo Magela e Silva*

Institute of Physics, University of Brasilia, Brasilia, 70.919-970, Brazil

ABSTRACT: Combining the one-dimensional tight-binding $\mathrm{Su}-$ Schrieffer-Heeger (SSH) model and the extended Hubbard model (EHM), the scattering of two oppositely charged bipolarons and a bipolaron-polaron pair is investigated under the influence of impurity effects using a nonadiabatic evolution method. These novel results for bipolarons show that the oppositely charged quasi-particles scatter into a mixed state composed of bipolarons and excitons. The excitation yield depends sensitively on the strength of the applied electric field. In the presence of an impurity, the critical electric field regime for formation of a state composed by bipolarons and excitons is increased. Additionally, we were able to obtain critical values of electric fields that played the role of drastically modifying the system dynamics. These facts suggest that the scattering between bipolarons and a bipolaron-polaron pair in the presence of impurities is crucial for the understanding of electroluminescence in optoelectronics devices, such as polymer light emitting diodes.

\section{INTRODUCTION}

Since the discovery of the light emitting properties on phenylbased organic semiconductors in the $90 \mathrm{~s}$, a huge growth of interest in the science and technology of conjugated polymers has been observed. The electronic and optical properties of conjugate polymers, together with their mechanical properties, processing advantages, versatility of chemical synthesis, and low cost make them particularly attractive materials for the electronics industry. There are many potential applications such as organic photovoltaics devices, ${ }^{1}$ thin-film transistors, ${ }^{2}$ and polymer light-emitting diodes (PLEDs). ${ }^{3}$ Especially, the characteristic to form thermally stable thin films as poly $(p$ phenylenevinylene) (PPV), which has high photoluminescence yields, makes these materials attractive for the development of PLEDs. $^{4,5}$ A PLED normally consists of a luminescent conjugate polymer layer, introduced between two metal electrodes. Electrons and holes are injected from the electrodes into the polymer layer and, as a result, this process induce selflocalized electron states called polarons. A polaron has spin $\pm 1 / 2$ and a charge $\pm e$. It is know that the injected electrons and holes forms electron-polarons and hole-polarons due to the strong electron-lattice interactions in these materials. Bipolarons, that are spinless charge carriers and possess charge $\pm 2 e$, can be created in PLEDs when the charge injection results in a large concentration of polarons. For example, two acoustic polarons with the same charge and antiparallel spins can combine with each other to for an acoustic bipolaron.

When an electron-bipolaron meets a hole-bipolaron, they may collide and recombine to form a mixed state composed by bipolarons and excitons, in which the electron and the hole are bonded in a self-trapped lattice deformation known as biexciton or bipolaron-exciton, in analogy to conventional excitons in inorganic semiconductors. The photon emission results from the radiative decay of the excitations. Thus, the yield of these excitations determines the electroluminescence efficiency in conjugated polymers. ${ }^{7,8}$ It is known that the scattering process between oppositely charged carriers and between charge carries and excitons plays an important role in the electroluminescence of PLEDs. ${ }^{9-12}$ Also, it has been generally accepted that the electron-electron interactions and the presence of impurities in a conjugated polymer lattice critically affects the polaron and bipolaron dynamics. ${ }^{13-17}$ Thus, the scattering process of oppositely charged bipolarons and bipolaron-polaron pair in the presence of impurities and the consequent yield of neutral excitations are believed to be of fundamental importance for PLEDs. Nevertheless, studies that take into account this physical picture remain not well described in the literature.

Another missing feature in this field of research is a complete understanding of the effects of the Hubbard type electronelectron $(e-e)$ interactions, together with impurity effects, on the mixed state of bipolarons and excitons (biexcitons) formation. The excited polaron decay due to this kind of interaction between bipolarons and polarons is crucial for the design of more efficient devices with respect to the electroluminescence and requires a better phenomenological description.

Some relevant theoretical studies carried out by $\mathrm{Di}^{18}$ and by $\operatorname{Sun}^{19}$ and co-workers have shown that external electric field

Received: March 26, 2013

Revised: August 14, 2013

Published: August 16, 2013 
strength has a significant influence on the results of bipolaronbipolaron and bipolaron-polaron interactions via scattering processes in conjugated polymers. Di and collaborators used a modified version of the Su-Schrieffer-Heeger (SSH) model to include the Brazoviskii-Kirova symmetry breaking term, $e-e$ interactions, and an external electric field to investigate the scattering and combination of oppositely charged bipolarons in a conjugated polymer monolayer and in PLED heterojunction systems. Their results show that two bipolarons can scatter into singlet biexciton state in both monolayer and/or multiple-layer electroluminescence polymeric materials. The yield of biexcitons in the collision of bipolarons can reach values as high as about $75 \%$. The biexciton state can decay to an exciton state, which can subsequently decay to the ground state, allowing the overall electroluminescent quantum efficiency in PLEDs from all sources to reach values as high as about $75 \%$. These results contributed to the understanding of the experimental fact that the internal quantum efficiency can reach $60 \%$. Using a similar approach, Sun and collaborators investigated the scattering processes between a negative polaron and a positive bipolaron in a conjugated polymer chain. Their results show that, initially, the negative polaron and the positive bipolaron are accelerated by the applied external electric field. A critical electric field is found corresponding to different behavior on polaron and bipolaron collision dynamics. Below the critical electric field, the polaron and bipolaron can scatter into an excited polaron with high yield. The excited polaron can decay to the polaron state through the emission of a photon. Above the critical electric field, the polaron and bipolaron will pass through each other and continue moving as independent entities. From these works it is possible to note that the products after the bipolaron-bipolaron and bipolaron-polaron interactions show a contribution to electroluminescence in conjugated polymers. Nevertheless, a consistent study about the products of these collisional processes, particularly concerning bipolarons as quasi-particles with respect to features that might increase the critical electric field for the formation of these excitations, had not been performed, so that further investigations are needed.

In this paper, a systematic numerical investigation of impurity effects on bipolaron-bipolaron and bipolaron-polaron scattering was performed in a cis-polyacetylene chain in terms of a nonadiabatic evolution method. The scattering of oppositely charged bipolarons and a bipolaron-polaron pair is investigated on a conjugated polymer chain subjected to different electric field strengths, on-site, and nearest-neighbor Coulomb interactions. Ehrenfest molecular dynamics was performed by using a one-dimensional tight-binding model including lattice relaxation. Combined with the extended Hubbard model (EHM), an extended version of the SSH model was used to include external electric fields and Brazoviskii-Kirova symmetry breaking term. The aim of this work is to give a physical picture of the products and their yields due to the scattering of a bipolaron-bipolaron and a bipolaron-polaron pair in conjugated polymers, when the electron-electron interactions and impurity effects are taken into account, and contribute to the understanding of these important processes, that may provide guidance for improving the electroluminescence efficiency in PLEDs.

\section{METHODOLOGY}

A polyacetylene chain in cis configuration ${ }^{20}$ was used to study collision between oppositely charged bipolarons and polaron- bipolaron pairs under the influence of impurity effects on conjugated polymers. The overall Hamiltonian is given by

$$
H_{\text {total }}=H_{\mathrm{SSH}}+H_{e e}+H_{\text {imp }}
$$

The first term in eq 1 is the SSH-type Hamiltonian modified to include an external electric field and the Brazovskii-Kirova symmetry-breaking, which has the following form:

$$
H=-\sum_{n, s}\left(t_{n, n+1} C_{n+1, s}^{\dagger} C_{n, s}+h c\right)+\sum_{n} \frac{K}{2} y_{n}^{2}+\sum_{n} \frac{p_{n}^{2}}{2 M}
$$

were $n$ indexes the sites of the chain. The operator $C_{n, s}^{\dagger}\left(C_{n, s}\right)$ creates (annihilates) a $\pi$-electron state at the $n$th site with spin $s ; K$ is the harmonic constant that describes a $\sigma$ bond, and $M$ is the mass of a $\mathrm{CH}$ group. The parameter $y_{n}$ is defined as $y_{n} \equiv$ $u_{n+1}-u_{n}$ where $u_{n}$ is the lattice displacement of an atom at the $n$th site. $p_{n}$ is the conjugated momentum to $u_{n}$, and $t_{n, n+1}$ is the hopping integral, given by

$$
t_{n, n+1}=\mathrm{e}^{-i \gamma \mathbf{A}(t)}\left[\left(1+(-1)^{n} \delta_{0}\right) t_{0}-\alpha y_{n}\right]
$$

where $t_{0}$ is the hopping integral of a $\pi$-electron between nearest neighbor sites in the undimerized chain, $\alpha$ is the electronphonon coupling, and $\delta_{0}$ is the Brazovskii-Kirova symmetrybreaking term, which is used to take the cis symmetry of the polymer into account. $\gamma \equiv e a /(\hbar c)$, with $e$ being the absolute value of the electronic charge, $a$ is the lattice constant, and $c$ is the speed of light. The relation between the time-dependent vector potential $\mathbf{A}$ and the uniform electric field $\mathbf{E}$ is given by $\mathbf{E}$ $=-(1 / c) \dot{\mathbf{A}}$.

The second contribution in eq 1 denotes the $e-e$ interactions and can be written as

$$
\begin{aligned}
H_{e e}= & U \sum_{i}\left(C_{i, \uparrow}^{\dagger} C_{i, \uparrow}-\frac{1}{2}\right)\left(C_{i, \downarrow}^{\dagger} C_{i, \downarrow}-\frac{1}{2}\right) \\
& +V \sum_{i}\left(n_{i}-1\right)\left(n_{i+1}-1\right)
\end{aligned}
$$

where $U$ and $V$ are the on-site and nearest-neighbor Coulomb repulsion strengths, respectively, and $n_{i}=C_{i, \uparrow}^{\dagger} C_{i, \uparrow}+C_{i, \downarrow}^{\dagger} C_{i, \downarrow}$.

The last contribution in eq 1 represents the one-site impurity effects and can be written in the form

$$
H_{\mathrm{imp}}=Z_{j} C_{j, s}^{\dagger} C_{j, s}
$$

$Z_{j}$ is the strength of an impurity, which is located at the $j$ th site. The parameters used here are $t_{0}=2.5 \mathrm{eV}, M=1349.14 \mathrm{eV} \times$ $f_{s}^{2} / \AA^{2}, K=21 \mathrm{eV} \AA^{-2}, \delta_{0}=0.05, \alpha=4.1 \mathrm{eV} \AA^{-1}, a=1.22 \AA$, and a bare optical phonon energy $\hbar \omega_{Q}=\hbar(4 K / M)^{1 / 2}=0.16 \mathrm{eV}$. These values have been used in previous simulations and are expected to be valid for conjugated polymers in general. ${ }^{21-23}$

In order to solve these equations numerically, first a stationary state that is self-consistent with all degrees of freedom of the system (the lattice plus electrons) is obtained. Then, the time evolution of the system is described by the equations of motion. The electronic wave function is the solution of the time-dependent Scrhödinger equation: 


$$
\begin{aligned}
& i \hbar \dot{\psi}_{k, s}(i, t)=-\left[t_{i, i+1}^{*}+V \tau_{s}(i, t)\right] \psi_{k, s}(i+1, t) \\
& \quad-\left[t_{i-1}, i+V \tau_{s}^{*}(i-1, t)\right] \psi_{k, s}(i-1, t) \\
& \quad+\left\{U\left[\rho_{-s}(i, t)-\frac{1}{2}\right]+Z_{j}\right. \\
& \left.\quad+V \sum_{s^{\prime}}\left[\rho_{s^{\prime}}(i+1, t)+\rho_{s^{\prime}}(i-1, t)-1\right]\right\} \psi_{k, s}(i, t)
\end{aligned}
$$

where $k$ is the quantum number that specifies an electronic state,

$$
\rho_{s}(i, t)=\sum_{k} \psi_{k, s}^{*}(i, t) \psi_{k, s}(i, t)
$$

and

$$
\tau_{s}(i, t)=\sum_{k} \psi_{k, s}^{*}(i+1, t) \psi_{k, s}(i, t)
$$

The equation of motion that describes the site displacement and provides the temporal evolution of the lattice is obtained by a classical approach. ${ }^{20}$ The nuclear dynamics is made with the Euler-Lagrange equations

$$
\frac{\mathrm{d}}{\mathrm{d} t}\left(\frac{\partial\langle L\rangle}{\partial \dot{u}_{n}}\right)-\frac{\partial\langle L\rangle}{\partial u_{n}}=0
$$

where

$$
\langle L\rangle=\langle T\rangle-\langle V\rangle
$$

Equation 9 leads to

$$
M \ddot{u}_{n}=F_{n}(t)
$$

with

$$
\begin{aligned}
& F_{n}(t)=M \ddot{u}_{n}=-K\left[2 u_{n}(t)-u_{n+1}(t)-u_{n-1}(t)\right] \\
& \quad+\alpha\left[B_{n, n+1}-B_{n-1, n}+B_{n+1, n}-B_{n, n-1}\right]
\end{aligned}
$$

where, $F_{n}(t)$ represents the force on the $n$th site. Here,

$$
B_{n, n}=\sum_{k, s} \psi_{k, s}^{*}(n, t) \psi_{k, s}\left(n^{\prime}, t\right)
$$

is the term that couples the electronic and lattice solutions. The primed summation represents a sum over the occupied states. By introducing instantaneous eigenstates, the solutions of the time-dependent Scrhödinger equation can be put in the form ${ }^{24}$

$$
\begin{aligned}
\psi_{k, s}\left(n, t_{j+1}\right)= & \sum_{l}\left[\sum_{m} \phi_{l, s}^{*}\left(m, t_{j}\right) \psi_{k, s}\left(m, t_{j}\right)\right] \\
& \times e^{\left(-i \varepsilon_{l} \Delta t / \hbar\right)} \phi_{l, s}\left(n, t_{j}\right)
\end{aligned}
$$

$\left\{\phi_{l}(n)\right\}$ and $\left\{\varepsilon_{l}\right\}$ are the eigenfunctions and the eigenvalues of the electronic part for the Hamiltonian at a given time $t_{j}$. Equation 12, which govern the evolution of system, may be numerically integrated using the method ${ }^{24}$

$$
\begin{aligned}
& u_{n}\left(t_{j+1}\right)=u_{n}\left(t_{j}\right)+\dot{u}_{n}\left(t_{j}\right) \Delta t \\
& \dot{u}_{n}\left(t_{j+1}\right)=\dot{u}_{n}\left(t_{j}\right)+\frac{F_{n}\left(t_{j}\right)}{M} \Delta t
\end{aligned}
$$

Hence, the electronic wave functions and the lattice displacements at the $(j+1)$ th time step are obtained from the $j$ th time step. At time $t_{j}$ the wave functions $\left\{\psi_{k, s}\left(i, t_{j}\right)\right\}$ can be expressed as a series expansion of the eigenfunctions $\left\{\phi_{l, s}\right\}$ at that moment:

$$
\psi_{k, s}\left(i, t_{j}\right)=\sum_{l=1}^{N} C_{l, k}^{s} \phi_{l, s}(i)
$$

where $C_{l, k}^{s}$ are the expansion coefficients. The occupation number for eigenstate $\phi_{l, s}$ is

$$
\eta_{l, s}\left(t_{j}\right)=\sum_{k}\left|C_{l, k}^{s}\left(t_{j}\right)\right|^{2}
$$

$\eta_{l, s}\left(t_{j}\right)$ describes the redistribution of electrons among the energy levels.

\section{RESULTS}

We carry out a systematic numerical investigation on impurities effects and $e-e$ interactions influence over the collision dynamics of bipolaron-bipolaron and bipolaron-polaron pairs in systems composed of 200-site cis-polyacetylene chain. For the electric field, turned on quasi-adiabatically, the values used in the simulations varied from 0.1 to $1.0 \mathrm{mV} / \AA$ with a increment of $0.1 \mathrm{mV} / \AA$ and from 1.0 to $2.5 \mathrm{mV} / \AA$ with a increment of $0.5 \mathrm{mV} / \AA$, whereas the on-site $e-e$ interactions values considered are $0.1,0.2,0.3,0.4$, and $0.5 \mathrm{eV}$. The nearestneighbor Coulomb repulsion strength was defined using the relation $V=U / 2$. In this context, Figure 1 presents the

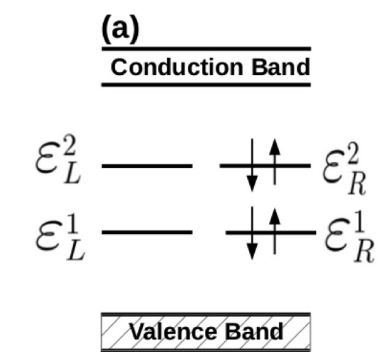

(b) Conduction Band

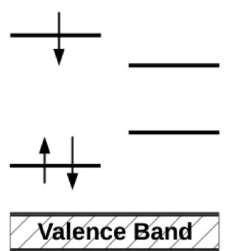

Figure 1. Energy-level schematic diagram of (a) hole-bipolaron (left) and electron-bipolaron (right) and (b) electron-polaron (left) and hole-bipolaron (right).

schematic diagram of energy-levels for the configurations investigated: bipolaron-bipolaron and bipolaron-polaron pair. In Figure 1(a) we have a single polymer chain containing a hole-bipolaron, represented by the absence of electrons in the $\varepsilon_{\mathrm{L}}$ levels, which yields a $+2 e$ charge, and an electronbipolaron, represented by the full occupation of the $\varepsilon_{\mathrm{R}}$ levels thus leading to $a-2 e$ charge. Figure $1(\mathrm{~b})$, on the other hand, represents another kind of system in which the left partially occupied levels are characteristic of a electron-polaron, whereas the right fully occupied levels represent a holebipolaron energy levels configuration. One can see the characteristic larger narrowing of the bipolarons energy levels when compared to those of the polaron.

A number of simulations were performed in which we varied not only the kind of quasi-particle collision but also the electric field, the Hubbard interaction, and the absence or presence of the impurity. In order to better present our results, we divided this section into three subsections, each one dealing with some sort of simulation, namely the colision between bipolarons in a chain free of other defects, presented in subsection I, the influence of impurity over the bipolarons collision, in 

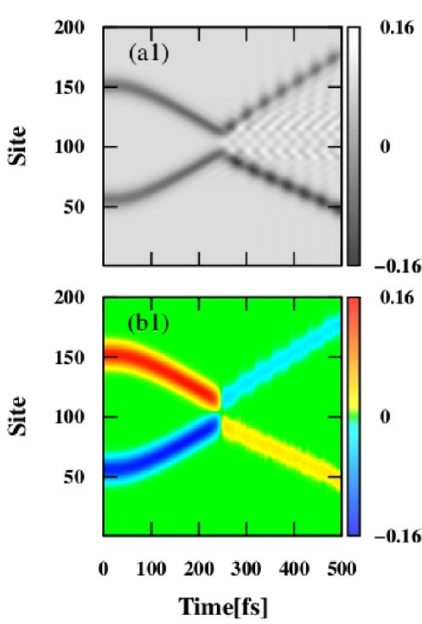

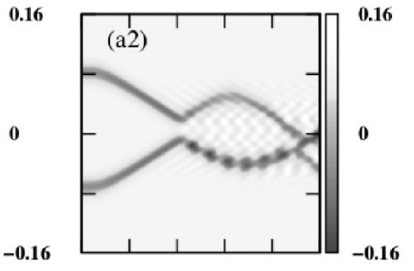

0.16

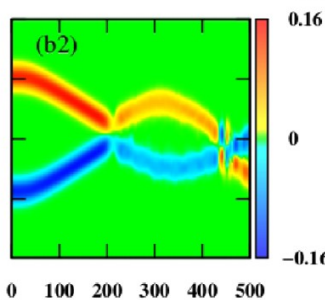

Time[fs]

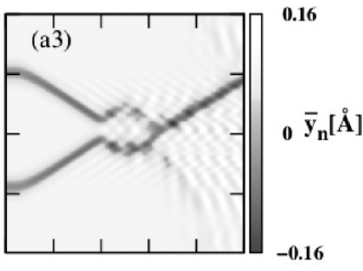

0.16

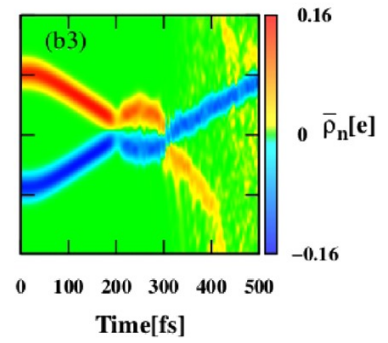

Figure 2. Bipolarons collision on a pristine chain for $U=0.1 \mathrm{eV}$. Order parameter (a) and charge density (b) time evolution. (a1-b1) $1.0 \mathrm{mV} / \AA$, (a2-b2) $2.0 \mathrm{mV} / \AA ̊$, and (a3-b3) $2.5 \mathrm{mV} / \AA$. .

subsection II, and the polaron-bipolaron collisional process is discussed in subsection III. Also for the sake of clarity, we carry out our analysis on the quasi particles dynamics by considering the mean charge density $\bar{\rho}(t)$, derived from the charge density $\rho(t)=\sum_{k, s}{ }^{\prime} \psi_{k, s}^{*}(n, t) \psi_{k, s}(n, t)$, and the mean order parameter $\bar{y}(t)$. The expressions for these quantities are given by $\bar{\rho}(t)=1-$ $\left[\rho_{n-1}(t)+2 \rho_{n}(t)+\rho_{n+1}(t)\right] / 4$ and $\bar{y}(t)=(-1)^{n}\left[y_{n-1}(t)-\right.$ $\left.2 y_{n}(t)+y_{n+1}(t)\right] / 4$. The goal is to provide a better visualization of the simulations and consequently to perform a more accurate analysis of the results.

I. Bipolaron-Bipolaron Collision on Pristine Chain. We begin our discussion by considering the simulation concerning the collision between two oppositely charged bipolarons in a chain free of defect, presented in Figure 2. The upper part of Figure 2 ((a1), (a2), and (a3)) represent the order parameter time evolution. One can confirm this by noting the blurring of the figure after the collision, which is a direct manifestation of created phonons. The bottom part of the this figure, on the other hand, presents the charge density pattern ((b1), (b2), and (b3)). As the color scale shows, we have a positive (red) structure initially in site 150 and a negative (blue) particle in the 50th site. The electric field brings the structures together and a collision takes place at varying time, depending on the applied field. This mechanism and the labeling notation is repeated throughout this work for different simulations.

In the present set of simulations we consider a Hubbard interaction of $0.1 \mathrm{eV}$ and different electric fields: $1.0 \mathrm{mV} / \AA$ ((a1) and (b1)), $2.0 \mathrm{mV} / \AA ̊$ in $((\mathrm{a} 2)$ and (b2)) and $2.5 \mathrm{mV} / \AA$ in $((\mathrm{a} 3)$ and (b3)). The bipolaron dynamics before the collision processes is quite similar to the polaron motion described by Stafström. ${ }^{25}$ The first interesting feature noted by an overall view of Figure 2 is the fact that, for these regimes, the bipolarons always passes through each other after the collision. This behavior is not observed when a collisional process of a polaron-pair are taken into account, for in those systems the polaron-pair is annihilated for electric fields greater than 1.2 $\mathrm{mV} / \AA^{12}$ We can note that the least energetic situations of Figure 2(a1) and Figure 2(b1) correspond to a scattering process. It is important to comment on, however, the difference between the emerging structures after the collision. It is noted, in Figure 2(b1), that the negatively charged excitation has a greater mobility than the positive one. This feature is to be further exploited, and can be more clearly understood in terms of a occupation number analysis, to be addressed shortly in this subsection. Another characteristic of the simulation performed in this case, that justifies this interesting and unexpected behavior, is the fact that a small value of impurity $(-0.02 \mathrm{eV})$ was used in 150th site to facilitate the composition of a oppositely charged bipolaron system. The impurity causes a small difference between the charge coupled to the bipolaron lattice defects, decreasing the charge concentration in the electron-bipolaron deformation. As a result, this reduction makes the structure to be faster. It should be noted that, even thought the presence of this impurity was only meant to generate the initial negative bipolaron solution, its effect lingers throughout the whole simulation. As a consequence we obtain a positive bipolaron with a slightly lower absolute charge than the corresponding negative one. The fact that these bipolarons are not identical reflects on the difference on the dynamical behavior of the structures. Particularly after the collision, when the presence of the phonon modes helps enhancing the difference on the structure trajectories, one can see that their paths are not perfectly mirrored. Also, depending on the applied electric field, more than one collision is necessary to accomplish this dynamics (Figure 2(b2) and Figure 2(b3)). In fact, a greater level of bipolarons interaction is perceived in these cases in which the electric field and the Hubbard interaction level are sufficiently large to partially overcome the large particles inertia, creating a transition structure that is momentarily self-trapped around 100th site for about $200 \mathrm{fs}$ in the case of the simulation with $2.0 \mathrm{mV} / \AA$ and about 100 fs for $2.5 \mathrm{mV} / \AA$. Thus, we believe that after the conditions for creating this transition structure is achieved (which was not the case only for $1.0 \mathrm{mV} / \AA$ of Figure 2(a1) and Figure 2(b1)), the electric field tends to destabilize such structure, and to direct the system to the final state of one bipolaron passing through each other. The obtained result show that, although of similar nature, the simulation in Figure 2(a2) and Figure 2(a3) possess as products different structures: whereas in the former case, both original structures are present, in the latter, only the electron-bipolaron remains stable. 

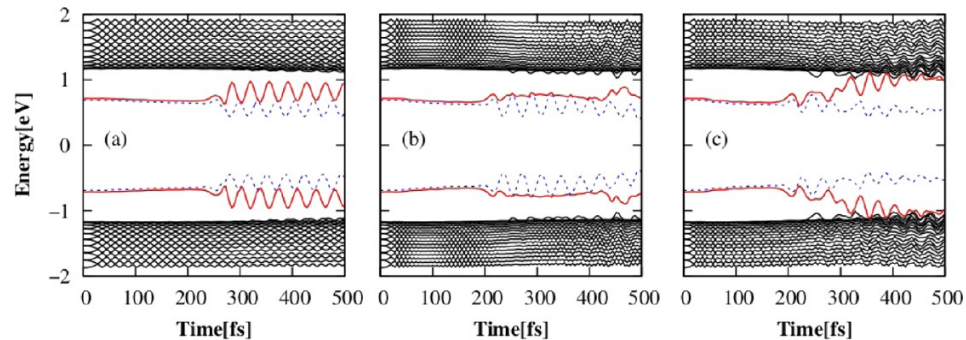

Figure 3. Energy levels time evolution for the bipolarons collision in a pristine chain for the cases showed in (a) Figure 2(a), (b) Figure 2(b), and (c) Figure 2(c).
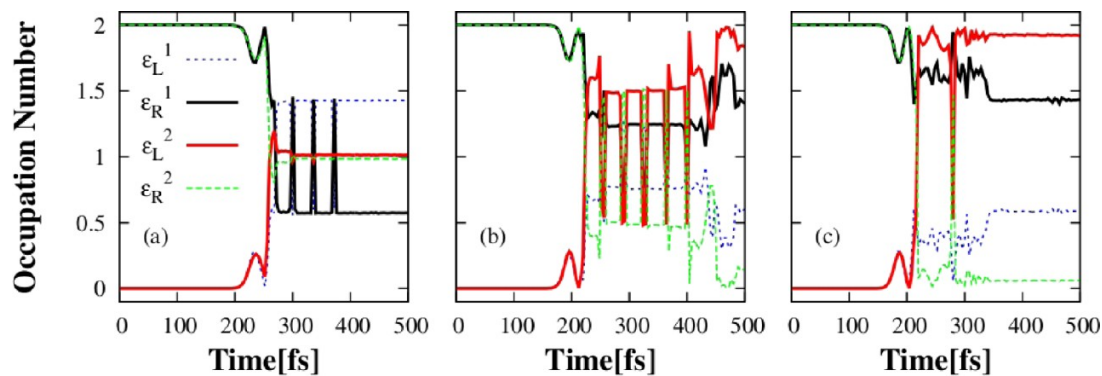

Figure 4. Occupation number time evolution for the bipolarons collision in a pristine chain for the cases showed in (a) Figure 2(a), (b) Figure 2(b), and (c) Figure 2(c).

Although we have just found a regime in which the quasiparticles destabilization is achieved, it is important to stress the great level of stability presented by the bipolaron themselves. Figure 3 presents the bipolarons energy levels time evolution for the cases presented in Figure 2. Although one can again note the oscillation pattern associated with the phonons after the collision, the presence of the states deep inside the energy gap is representative of the bipolaron integrity in Figure 2(a) and Figure 2(b). The disappearing of the structure in Figure 2(a3), corresponding to the blurring of Figure 2(b3) is an indication of the destabilization of the hole-bipolaron through the charge spread at the end of the simulation. The same feature can be exactly inferred by analyzing the red energy levels that returns to the valence and conduction band in Figure 3 (c), thus conducting to the vanishing of the bipolaron. It is important to remark that previous similar theoretical work performed on polarons have not yielded equivalent results in terms of the consistency of the quasi-particle, in the sense that polarons could not survive collisions such as those implemented in Figure 2(b). ${ }^{12}$ These considerations are consistent with the well-known fact that bipolarons are much more stable quasi-particles than polarons in conducting polymers.

Figure 4 presents the time evolution of the occupation numbers also for the cases presented in Figures 2 and 3. The fast and periodic exchange of levels between the electrons of levels $\varepsilon_{\mathrm{L}}^{1}$ and $\varepsilon_{\mathrm{R}}^{1}$ and between $\varepsilon_{\mathrm{L}}^{2}$ and $\varepsilon_{\mathrm{R}}^{2}$ is noted and might be considered to be an indication of the creation of different quasiparticles. However, these oscillations do not give rise to an amount of shift in the energy levels of sufficient magnitude to define an independent particle. The partial transference of electrons is noted by the fractional numbers achieved in the final states, which is a measure of the interference among each bipolarons electronic states. The exception stands for Figure 4(a), in which the structures pass through each other directly. The occupation numbers of Figure 4(a), suggest that both bipolarons remain stable after the collision. Even in this case, the oscillation pattern of these structures is a mere reflex of the phonons interaction that does not lead to the creation of a different particle. This conclusion is obtained through the analysis of a single electron occupying the lower levels inside the gap and some quantity near 1.5 electrons populating the levels deeper inside the gap. The difference on the kinematic behavior of the blue and red excitations in Figure 2 should be addressed for the difference in nature of these emerging quasiparticles. Concerning Figure 4(b,c), it is particularly interesting to observe the time length difference on the oscillating pattern of electrons level population, which is consistent to the lifetime of the transition structure previously mentioned. Although of similar nature, the results obtained in Figure 4(b,c) allow us to conclude that in the latter case, the creation of a neutral excitation derived from the bipolarons collision is fully accomplished.

It is important to mitigate the possible misleading feature that the scattering of bipolarons would always take place in this kind of simulation. Figure 5 presents the situation where simple scattering does not arise from bipolarons interaction. We considered an electric field and a Hubbard interaction of 0.5 $\mathrm{mV} / \AA$ and $0.2 \mathrm{eV}$ in Figure $5(\mathrm{a})$ and of $2.5 \mathrm{mV} / \AA$ and $0.4 \mathrm{eV}$, in Figure 5(b) respectively. The mechanics of the collision is the same as in the other simulations. In this case, however, the bipolarons are not suitably scattered. In Figure 5(a), the electric field is way too small to provide an effective collision in terms of bipolarons transference. We thus observe an approximately elastic collision between the bipolarons. In Figure 5(b), on the other hand, the collision gives rise to a neutral excitation, instead of the conventional scattering. Thus, instead of a typical collision as those proposed in Figure 2, we have the formation 


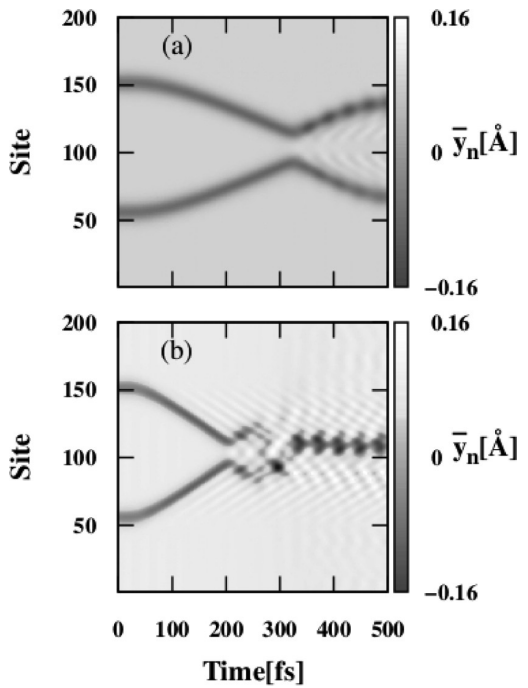

Figure 5. Order parameter time evolution in the cases: (a) Elastic collision between bipolarons for $0.5 \mathrm{mv} / \AA$ and $U=0.2 \mathrm{eV}$ and (b) biexciton formation for $2.5 \mathrm{mV} / \AA$ and $U=0.4 \mathrm{eV}$.

of a different neutral structure, henceforward denominated biexciton. ${ }^{18}$ This can be confirmed due to the fact that the resulting structure is not carried by the external electric field after the collision. We thus conclude that the Hubbard interactions and electric field regimes are of particular importance when considering each kind of particles interaction. It is important to observe that, for $U>0.4 \mathrm{eV}$, the mechanism of bipolarons recombination dominates, as described by Sun and Stafström, ${ }^{26}$ where the mixed start of bipolarons and excitons does not occur. Thus, in order to create a given type of structure out of a collisional process, several parameters must be carefully observed.

We finish the discussion of this subsection by presenting a schematic diagram for the energy levels that represents the products of the collisional process between the bipolaron-pair. Figure 6(a) represents the product formed by the scattering of the oppositely charged bipolarons, where two bipolaronexcitons are created. Figure 6(b) shows a case where only an excited bipolaron remains in the lattice. Due to the annihilation of one bipolaron, only two energy levels are present inside the bandgap. Finally, Figure 6(c) shows the energy level configuration when both charged bipolarons are annihilated (forming a neutral biexciton), for example, for a electric field of $2.5 \mathrm{mV} / \AA$ and $U=0.4 \mathrm{eV}$ (case showed in Figure 5(b)). This conclusions are obtained through an analysis of the occupation numbers time evolution. It is important to remark that what at first sight might appear to be the scheme of ground state systems, as might be the case of (b) and (c) systems, is, in fact, a representation of the different excited states that the system might reach after the collision. Although the electronic occupation is that of a fundamental state, we should remember that our nonlinear excitations arise from the coupling between the electronic charge and the lattice distortion, and not solely from the former. Thus, it is clear that, after the bipolarons collision, different excited states take place, as could be observed in Figure 2, for instance, that represent charge density and order parameter time evolution. As the parameters

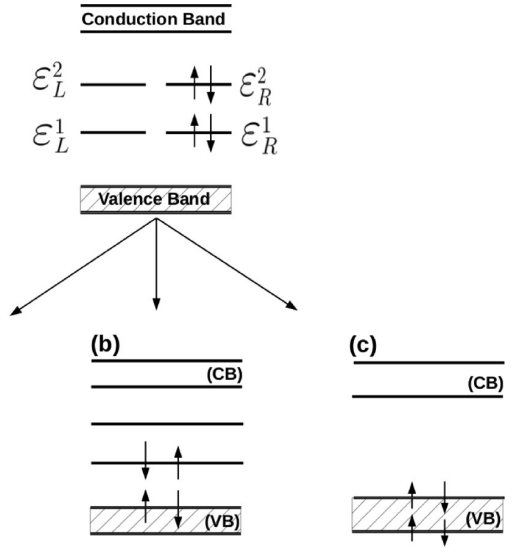

Figure 6. Possible results due to the collisional process of oppositely charged bipolarons. The conduction and valence bands are represented by $(\mathrm{CB})$ and $(\mathrm{VB})$ respectively.

used in all simulations discussed above are noted to dramatically change the dynamical behavior of the system, it is desired to know whether other properties such as the structural characteristic of the chain also affects the obtained results. In the next subsection we specifically deal with the role played by an impurity over the collision mechanism and the resulting structures.

II. Bipolaron-Bipolaron Collision: Impurity Effects. The first set of results presented in this subsection regards the same kind of simulation as in Figure 2, but this time with an impurity of $0.2 \mathrm{eV}$ placed at the 100th site. The goal is to investigate the influence this structure has over the system. In Figure 7(a1), (a2), and (a3) we considered electric field values of $1.0,1.5$, and $2.5 \mathrm{mV} / \AA$, and Hubbard interactions of $0.1,0.2$, and $0.3 \mathrm{eV}$, respectively. We can see in Figure $7(\mathrm{a} 1)$ and (b1) that the bipolarons passes through each other when subjected to an electric field of $1 \mathrm{mV} / \AA$. This results in a decrease of the critical field for transferring the bipolarons: from the $2.0 \mathrm{mV} / \AA$ condition on the pristine chain case to the presently discussed situation. In Figure 7(a2) and (b2), we note that, as we increase the electric field and the Hubbard interaction, the collision tends to favor the creation of a mixed state between bipolarons and excitons, through a mechanism similar to that of a transition structure discussed in the previous subsection. Nevertheless, in this case, we can already note that the emerging structure already has the exciton nature in its absence of net charge. This feature is clear in Figure $7(\mathrm{a} 2)$, where the order parameter path is shown to be static, in spite of the applied electric field. If we focus in Figure 7(a3) and (b3), we can note that a structure of the Figure $7(\mathrm{a} 3)$ with zero net charge (Figure $7(\mathrm{~b} 3)$ ) is present in the chain after the collision, but in this case, we observe the complete vanishing of the positively charged bipolaron and a much more stabilized localized bipolaron exciton state around site 65. Overall, if these results are compared to those of Figure 2, we can see that the impurity has acted in the sense of decreasing the necessary electric field to observe a proper bipolaron-exciton creation, and also led to a proper creation instead of a simple scattering of bipolarons.

Concerning the stability of the quasi-particles throughout the process, we can analyze Figure 8 in which a time evolution of 

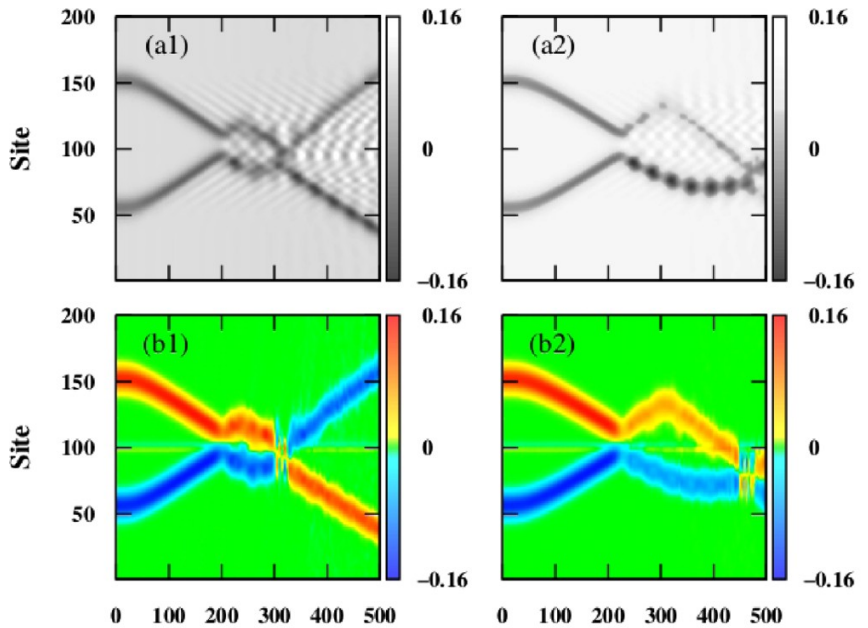

0.16

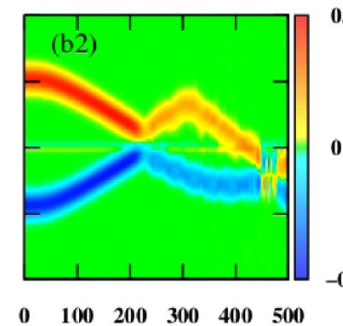

0.16
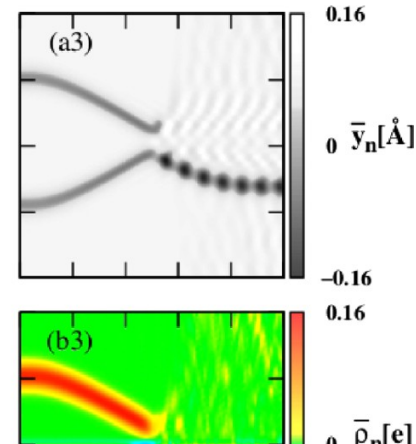

Time[fs]

Time[fs]

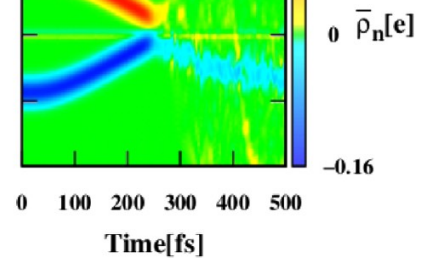

Figure 7. Bipolarons collision on an impurity $(0.2 \mathrm{eV})$ doped chain. Order parameter (a) and charge density (b) time evolution. (a1-b1) $1.0 \mathrm{mV} / \AA ̊$ and $U=0.1 \mathrm{eV} ;(\mathrm{a} 2-\mathrm{b} 2) 1.5 \mathrm{mV} / \AA$ and $U=0.2 \mathrm{eV} ;(\mathrm{a} 3-\mathrm{b} 3) 2.5 \mathrm{mV} / \AA$ and $U=0.3 \mathrm{eV}$.

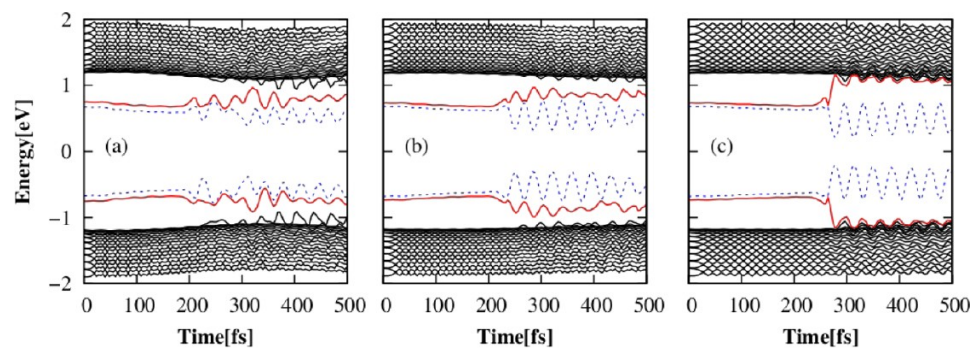

Figure 8. Energy level time evolution for the bipolarons collision in an impurity doped chain for the cases showed in (a) Figure 7(a), (b) Figure $7(\mathrm{~b})$, and $(\mathrm{c})$ Figure $7(\mathrm{c})$.
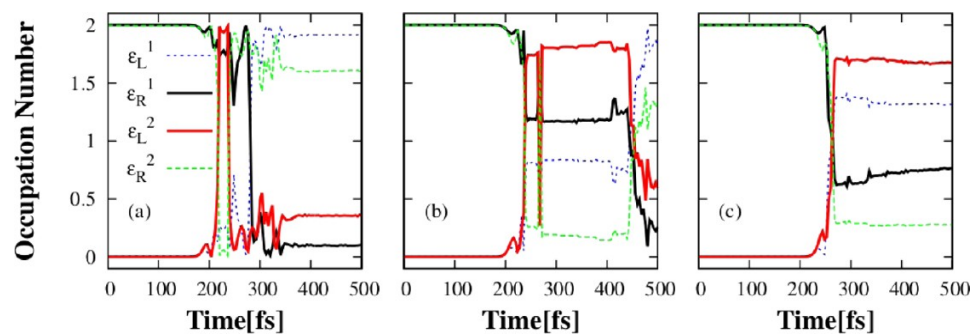

Figure 9. Occupation number time evolution for the bipolarons collision in an impurity doped chain for the cases showed in (a) Figure 7(a), (b) Figure 7(b), and (c) Figure 7(c).

the energy levels is presented. The systematic pattern of the red energy levels approaching the conduction and valence bands with the increasing of the electric field is a notorious signature of the disappearing of a bipolaron in the collisional process of excitons formation. So is the presence, albeit oscillating according to the phonon modes, of the blue levels corresponding to the other particle. Unlike Figure 8(a), Figure $8(\mathrm{~b})$ and (c) shows that the mean value of the oscillating levels is importantly increased in relation to the initial value. The conclusion is that, indeed, a new particle is created out from the collision process. This conclusion is confirmed by the occupation number analysis, presented in Figure 9. The hopping between levels is diminished as the electric field is increased, due to the fact that the exciton creation is favored in these regimes. Naturally, the exchange between electrons of each bipolaron levels tends to cease when these structures are no longer available for conduction, which is definitely the case of Figure 9(c), partially for Figure 9(b) and not quite for Figure 9(a). Since the yield of exciton creation particularly depends on this oscillation frequency and of the final occupation pattern, we have found regimes in which this efficiency of creation is 
higher (such as in Figure 9(c)) and others in which the efficiency is lower (such as Figure $9(a)$ ).

At this point we should again remark that the dynamical behavior observed in Figure 2 depend intrinsically on the electric field and Hubbard parameter used in the simulations. For instance, if we use $0.5 \mathrm{mV} / \AA$ for the electric field and a Hubbard interaction of $0.1 \mathrm{eV}$, we reach a situation in which a bipolaron is not able to pass through each other. Figure 10 is

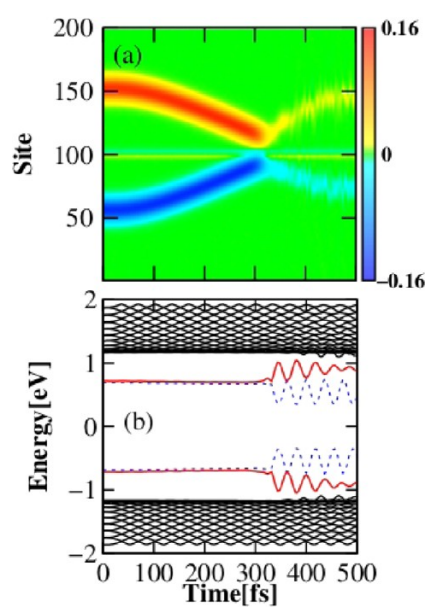

Figure 10. Bipolarons elastic collision with an impurity for $0.5 \mathrm{mV} / \AA$ and $U=0.1 \mathrm{eV}$ : (a) charge density time evolution and (b) energy levels time evolution.

meant to discuss this possibility. In Figure 10(a) we have the charge density order parameter for the case being, whereas Figure $10(\mathrm{~b})$ presents the time evolution for the energy levels. We can see what appears to be a quasi-elastic collision between the particle and the impurity. It results in the formation of a chargeless structure of the bipolaron-exciton type. The energy levels confirm this discussion through the positioning change of the particle levels inside the gap. The similarity between the results discussed for the previous case (Figure 10) to those presented in Figure 5(a) provides us with a tool to perform a fair comparison on the effects of impurity over the efficiency of the exciton creation. In order to do so, Figure 11 compares the results on occupation numbers for these situations with (Figure 11(a)) and without (Figure 11(b)) impurity on the chain. Since in both cases the bipolarons could not pass through each other, and the product is found to be of the same kind, an efficiency analysis is rather straightforward. Figure 11(a) (top) represents the occupation number analysis for the simulation in which an impurity is present in the polymer chain, whereas Figure 11(b) (bottom) is the equivalent simulation for the pristine chain. The periodic hopping of electrons between the levels is observed to be much more intense in the latter case, thus corresponding to a lower yield on the exciton efficiency. As a conclusion we have that, besides decreasing the electric field for exciton formation, another important effect impurity has over the system is to increase the efficiency of the creation these structures.

The schematic diagram for the energy levels that represents the products of the collisional process between the bipolaronpair, in a impurity doped chain, is similar to the configurations described in Figure 6. Since the impurity endowed systems are
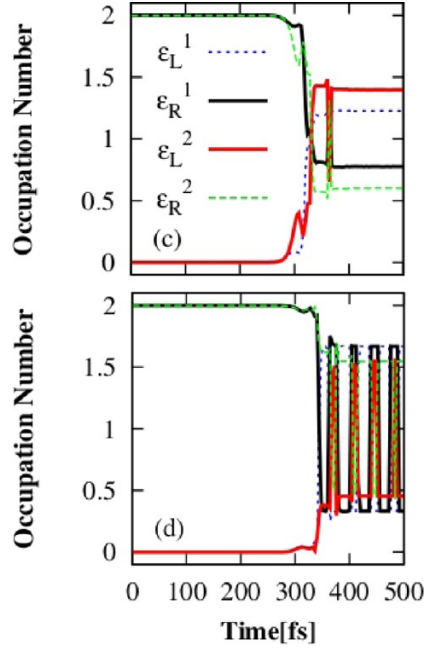

Figure 11. Bipolaron-exciton creation comparison: (a) in impurity doped chain for the case showed in Figure 10(a) $(0.5 \mathrm{mV} / \AA$ and $U=$ $0.1 \mathrm{eV}$ ) and (b) in a pristine chain for the case discussed in the previous subsection (Figure 5(a)) $(0.5 \mathrm{mV} / \AA ̊ \AA$ and $U=0.2 \mathrm{eV})$.

found to be superior in every studied sense as far as the excitons study is concerned, we decided to discuss, in the last subsection, the collisional process between different types of quasi-particles, namely, polarons and bipolarons, solely considering the situation of polymer chains in the presence of impurities. The energy levels for this system configuration is represented in Figure 1(b). The products, considering the bipolaron-polaron pair collision for pristine systems, are expected to be qualitatively the same as reported in the literature, $^{19}$ when the same Hubbard and electric field parameters were applied.

III. Polaron-Bipolaron Collision. In this last subsection we discuss the results concerning the collision between a polaron and a bipolaron in an $0.2 \mathrm{eV}$ impurity endowed chain. In this set of simulations we consider the collision between a electron-polaron and a hole-bipolaron. The Hubbard interaction was fixed to be $0.2 \mathrm{eV}$. Up to this point, we could observe a great deal of symmetry on the behavior of the charge carrier as a consequence of the symmetry of the used Hamiltonian. Figure 12, however, presents a very different picture. One can readily note the difference in the response between the polaron movement and that of the bipolaron. The reasoning should be obvious if we consider the difference between the masses and charges of these quasi-particles. Figure 12(a1) and Figure 12(b1) correspond to an applied field of 0.5 $\mathrm{mV} / \AA$. After around $450 \mathrm{fs}$, we can not observe, in Figure $12(\mathrm{a} 1)$, more than a single lattice distortion corresponding to a neutral complex system in Figure 12(b1). As a matter of fact, what happens is that in this regime, the structures become trapped by the impurity. Thus, in this case, instead of promoting the exciton creation, contributing to a system with better charge mobility, what happens is that the charge carriers loses their ability to follow the applied electric field, consequently yielding a system with low overall charge mobility. If we increase the electric field to the value of 1.0 $\mathrm{mV} / \AA$, the situation presented in Figure 12(a2) and (b2), the polaron is able to escape from the impurity potential and passes 

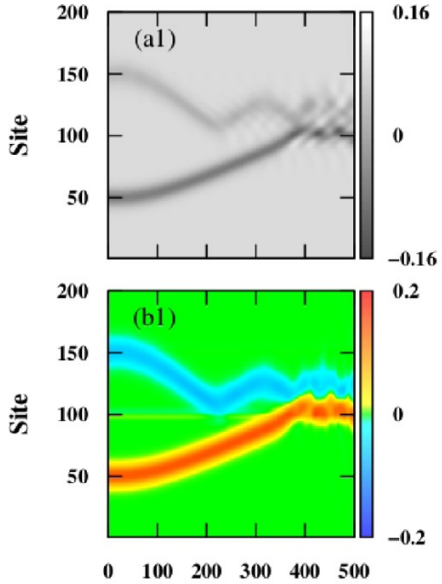

Time[fs]
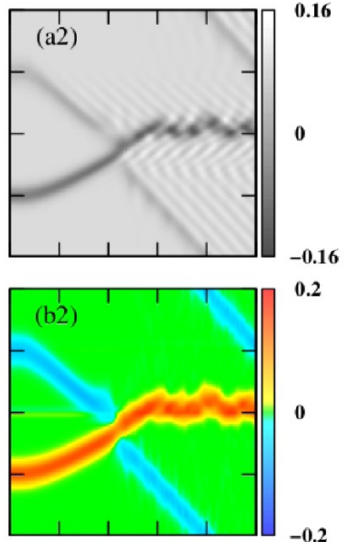

$\begin{array}{llllll}0 & 100 & 200 & 300 & 400 & 500\end{array}$

Time[fs]
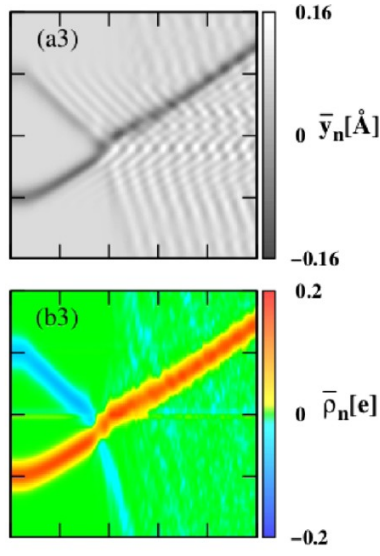

$\begin{array}{llllll}0 & 100 & 200 & 300 & 400 & 500\end{array}$

Time[fs]

Figure 12. Polaron-bipolaron collision on an impurity doped chain for $U=0.2 \mathrm{eV}$. (a) Order parameter and (b) charge density time evolution. $(\mathrm{a} 1-\mathrm{b} 1) 0.5 \mathrm{mV} / \AA$, $(\mathrm{a} 2-\mathrm{b} 2) 1.0 \mathrm{mV} / \AA$, and $(\mathrm{a} 3-\mathrm{b} 3) 2.0 \mathrm{mV} / \AA$
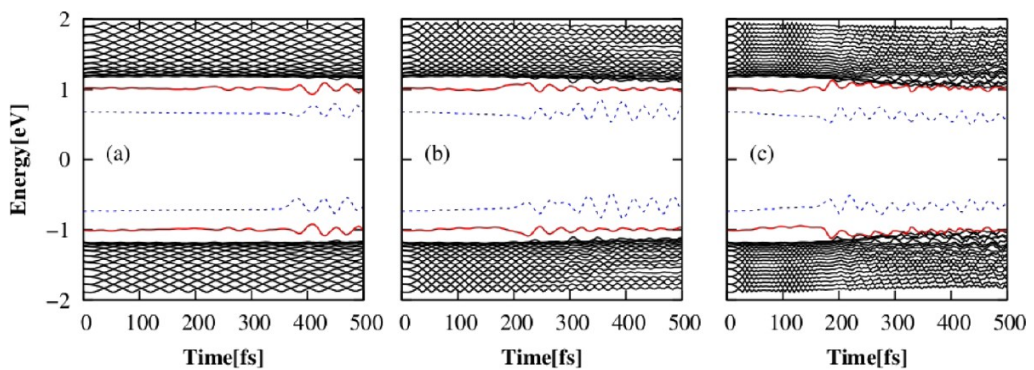

Figure 13. Energy level time evolution for the bipolaron-polaron collision in an impurity doped chain for the cases showed in (a) Figure 12(a), (b) Figure 12(b), and (c) Figure 12(c).
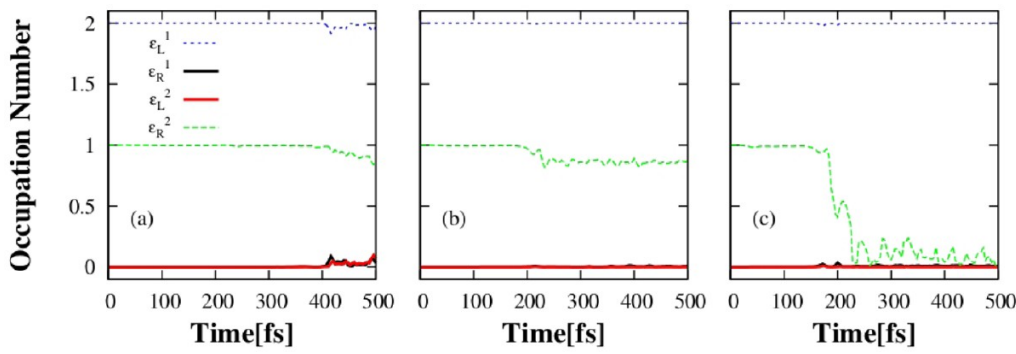

Figure 14. Occupation number time evolution for the bipolaron-polaron collision in an impurity doped chain for the cases showed in (a) Figure 12(a), (b) Figure 12(b), and (c) Figure 12(c).

through the bipolaron, which remains trapped. We can see that, even in this case, both structures remain stable both in their order parameter and in their charge density profile. A further increase on the electric field to the value of $2.0 \mathrm{mV} / \AA$ (Figure $12(\mathrm{c} 1)$ and (c2)) gives rise not only to the bipolaron release from the impurity, but also to the annihilation of the polaron, a feature that can be noted both in the order parameter and the charge density time evolution. This fact finds complete support to the already mentioned property of bipolarons being more stable structures than polarons.
Again, this analysis on the loss of stability and the kind of quasi-particle present in the system is better accomplished by studying the time evolutions of the energy levels, which is accomplished in Figure 13. The polaron levels are represented by the red lines and those of the bipolarons, by the blue lines. The aforementioned loss of stability of the polaron is observed by the reaching of the red levels in Figure 13(c) to the valence and conduction bands. This same reasoning can be obtained by studying the occupation number evolution in Figure 14. One can note in Figure 14(c) the vanishing of a polaron by the emptying of the electron in the $\varepsilon_{\mathrm{R}}^{2}$ state. In this case, the 
electron leaves the state inside the gap reaching the lowest unoccupied molecular orbital (LUMO), which characterizes the quasi-particle destabilization. One can observe that in Figure 13(a) and Figure 13(b), both structures are present throughout the simulation.

An interesting feature worth to discuss in the present paper is the role played by phonons in the dissociation of polarons. Figure 12 taught us that the collision carried out at such an energetic situation as a $2.0 \mathrm{mV} / \AA$ would lead to the polaron destruction, whereas at $1.0 \mathrm{mV} / \AA$, the structure maintained its integrity. We chose to present in Figure 15 an intermediate
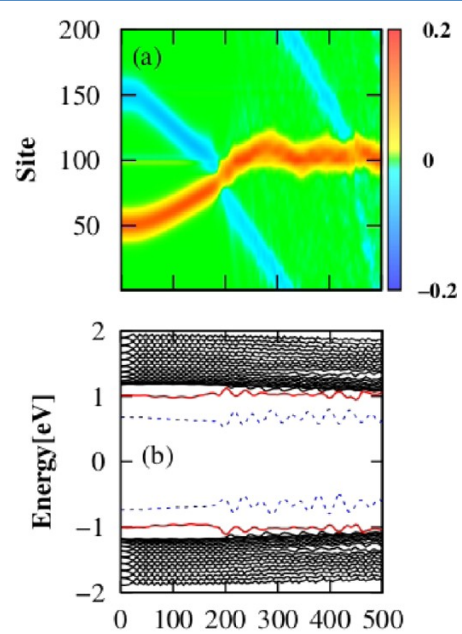

Time[fs]

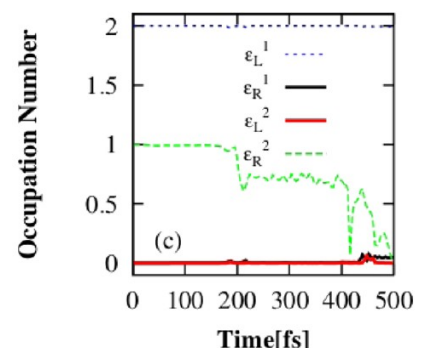

Figure 15. Multiple collisions between the bipolaron-polaron pair for $1.5 \mathrm{mV} / \AA$ and $U=0.2 \mathrm{eV}$. (a) charge density, (b) energy levels, and (c) occupation number time evolution.

field value of $1.5 \mathrm{mV} / \AA$ to investigate the effect of the collision itself on the stability of the carrier, for $U=0.2 \mathrm{eV}$. It is clear from Figure 15(a) that, in this case there are two successive collisions between the polaron and the bipolaron. It is important to note that the two collisions take place due to the periodic boundary conditions imposed to our polymer chains. By the blurring of the blue structure, one can see the relative spreading of the polaron charge after the first collision. However, the structure arises from this collision with its collective behavior unaffected. Indeed, a quick glimpse onto Figure 15(b) assures that between the two collisions, the polaron state can still be recognized. After the second collision, however, the spreading of the polaron charge is completed, as can be seen in the charge density profile of Figure 15(a) and confirmed by the returning of the polaron levels to the highest occupied molecular orbital (HOMO) and LUMO, in Figure 15(b). We conclude that the interaction with the lattice phonons created from both collisions add up to help destabilize the polaron. Another property that matches with the previous results is the integrity of the bipolaron, which remains trapped to the impurity since the first collision. All these features can be summarized by studying the occupation numbers evolution in Figure $15(\mathrm{c})$. We can recognize the disturbance of the $\varepsilon_{\mathrm{R}}^{2}$ after $200 \mathrm{fs}$, when the first collision takes place, and the complete evacuation of this level after the second collision, which characterizes the polaron destruction, through the lattice vibration.

We finish our discussion by presenting a schematic diagram for the energy levels that represents the products of the collisional process between the bipolaron-polaron pair. Figure 16(a) represents the energy levels configuration of the

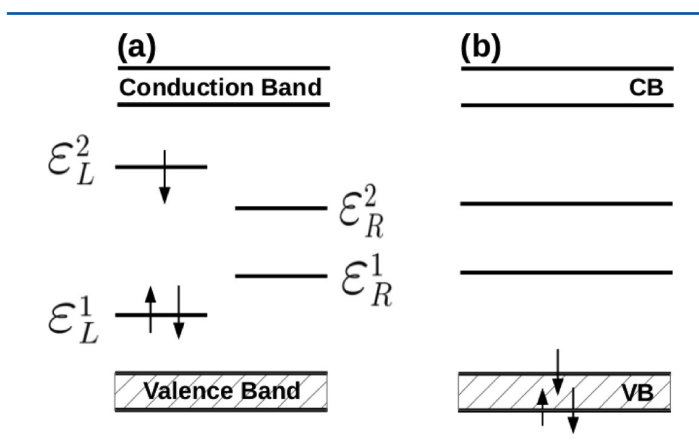

Figure 16. Possible results due to the collisional process of a bipolaron-polaron pair. The conduction and valence band are represented by $(\mathrm{CB})$ and $(\mathrm{VB})$ respectively.

bipolaron-polaron pair when both quasi-particles keeps the integrity. This configuration represents the cases showed in Figure 12(a) and Figure 12(b). Figure 16(b) shows a case where only a hole-bipolaron remains in the lattice. Due to the annihilation of the electron-polaron, only two energy levels (that represents a hole-bipolaron configuration) are present inside the bandgap. Such energy levels configuration represent the cases discussed in Figure 12(c) and Figure 15.

\section{CONCLUSIONS}

In summary, we developed a modified version of the SSH model to include an external electric field, the BrazovskiiKirova symmetry breaking term, on-site and nearest-neighbor Coulomb interactions, and impurities in order to investigate the effects of these properties over the collision process of oppositely charged bipolarons and bipolaron-polaron pairs in cis-symmetry conducting polymers chains. Using a nonadiabatic evolution method, within a one-dimensional tight-binding model, the critical electric field regimes for formation of a mixed state compose by bipolarons and excitons was presented. The influence of electron-electron interactions on the products formed after the bipolaron-bipolaron and bipolaron-polaron collision was also discussed. Furthermore, it is found that the electron-electron interactions plays an important role on the yield of excitations after the scattering between the charge carriers in both configurations. The charge coupling to the lattice deformation after the scattering is sensitive to on-site an nearest-neighbor Coulomb interaction in the sense that, for higher values of $U$, less charge remains 
coupled to the mixed state composed by bipolarons and excitons and the bipolaron-polaron pair. When impurity effects are taken into account, the maximum critical electric field for the formation of states composed of bipolarons and excitons increases compared to a system without impurity effects. Also, the results indicate that the presence of impurities in a conjugated polymer lattice, for all electric fields regimes, improves the excitation yield and favors the bipolaron-exciton formation. This fact may provide guidance for improving the electroluminescence yields in PLEDs.

\section{AUTHOR INFORMATION}

\section{Corresponding Author}

*E-mail: magela@fis.unb.br.

\section{Notes}

The authors declare no competing financial interest.

\section{ACKNOWLEDGMENTS}

The authors gratefully acknowledge the financial support from the Brazilian Research Councils CNPq, CAPES, and FINATEC.

\section{- REFERENCES}

(1) Sun, Y.; Welch, G. C.; Leong, W. L.; Takacs, C. J.; Bazan, G. C.; Heeger, A. J. Solution-processed small-molecule solar cells with $6.7 \%$ efficiency. Nat. Mater. 2012, 11, 44-48.

(2) Braga, D.; Erickson, N. C.; Renn, M. J.; Holmes, R. J.; Frisbie, C. D. High-transconductance organic thin-film electrochemical transistors for driving low-voltage red-green-blue active matrix organic lightemitting devices. Adv. Funct. Mater. 2012, 22, 1623-1631.

(3) Lu, L. P.; Kabra, D.; Friend, R. H. Barium hydroxide as an interlayer between zinc oxide and a luminescent conjugated polymer for light-emitting diodes. Adv. Funct. Mater. 2012, 22, 4165-4171.

(4) Burroughes, J. H.; Bradley, D. D. C.; Brown, A. R.; Marks, R. N.; Mackay, K.; Friend, R. H.; Burns, P. L.; Holmes, A. B. Light-emitting diodes based on conjugated polymers. Nature 1990, 347, 539-541. (5) Ho, P. K. H.; Kim, J. S.; Burroughes, J. H.; Becker, H.; Li, S. F. Y.; Brown, T. M.; Cacialli, F.; Friend, R. H. Molecular-scale interface engineering for polymer light-emitting diodes. Nature 2000, 404, 481484.

(6) Di, B.; Meng, Y.; Wang, Y. D.; Liu, X. J.; An, Z. Formation and evolution dynamics of bipolarons in conjugated polymers. J. Phys. Chem. B 2011, 115, 964-971.

(7) Braun, D.; Heeger, A. J. Visible light emission from semiconducting polymer diodes. Appl. Phys. Lett. 1991, 58, 1982-1984.

(8) Köhler, A.; Wilson, J. S.; Friend, R. H. Fluorescence and phosphorescence in organic materials. Adv. Mater. 2002, 14, 701-707.

(9) Sun, Z.; Liu, D. S.; Stafström, S.; An, Z. Scattering process between polaron and exciton in conjugated polymers. J. Chem. Phys. 2011, 134, 044906-5.

(10) Sun, Z.; Li, Y.; Li, D. S.; An, Z.; Xie, S. Scattering processes between bipolaron and exciton in conjugated polymers. Phys. Rev. B. 2009, 79, 201310-4.

(11) Li, Y.; Gao, K.; Sun, Z.; Yin, S.; Liu, D. S.; Xie, S. J. Intrachain polaron motion and geminate combination in donor-acceptor copolymers: Effects of level offset and interfacial coupling. Phys. Rev. B 2008, 78, 014304-13.

(12) An, Z.; Di, B.; Wu, C Q. Inelastic scattering of oppositely charged polarons in conjugated polymers. Eur. Phys. J. B 2008, 63, 7177.

(13) Yan, Y. H.; An, Z.; Wu, C. Q. Dynamics of polaron in a polymer chain with impurities. Eur. Phys. J. B 2004, 42, 157-163.

(14) Harigaya, K.; Terai, A. Metal-insulator transition in doped conjugated polymers: Effects of long-ranged Coulomb potentials. Phys. Rev. B 1991, 44, 7835-7843.
(15) Harigaya, K.; Wada, Y.; Fesser, K. Impurity distribution and electronic states in doped conjugated polymers in the coherentpotential approximation. Phys. Rev. B 1990, 42, 11303-11309.

(16) Di, B.; An, Z.; Li, Y. C.; Wu, C. Q. Effects of e-e interactions on the dynamics of polarons in conjugated polymers. Eur. Phys. Lett. 2007, 79, 17002-5.

(17) Zhao, H.; Chen, Y. G.; Zhang, X. M.; An, Z.; Wu, C. Q. Correlation effects on the dynamics of bipolarons in nondegenerate conjugated polymers. J. Chem. Phys. 2009, 130, 234908-4.

(18) Di, B.; Meng, Y.; Wang, Y. D.; Liu, X. J.; An, Z. Electroluminescence enhancement in polymer light-emitting diodes through inelastic scattering of oppositely charged bipolarons. J. Phys. Chem. B 2011, 115, 9339-9344.

(19) Sun, Z.; Li, Y.; Liu, D. S.; An, Z.; Xie, S. Dynamical study of polaron-bipolaron scattering in conjugated polymers. Org. Elec. 2010, $11,279-284$.

(20) Hegger, A. J. Nobel Lecture: Semiconducting and metallic polymers: Generation of polymeric materials. Rev. Mod. Phys. 2001, 73, $681-700$.

(21) Ribeiro, L. A.; de Oliveira Neto, P. H.; da Cunha, W. F.; Roncaratti, L. F.; Gargano, R.; da Silva Filho, D. A.; e Silva, G. M. Exciton dissociation and charge carrier recombination processes in organic semiconductors. J. Chem. Phys. 2011, 135, 224901-5.

(22) Ribeiro, L. A.; de Oliveira Neto, P. H.; da Cunha, W. F.; Gargano, R.; e Silva, G. M. Predicting the equilibrium structure of organic semiconductors with genetic algorithms. Chem. Phys. Lett. 2013, 555, 168-172.

(23) Ribeiro, L. A.; de Oliveira Neto, P. H.; da Cunha, W. F.; e Silva, G. M. Dynamics of Photogenerated Polaron-Excitons in Organic Semiconductors. Phys. Proc. 2012, 28, 112-116.

(24) Lima, M. P.; e Silva, G. M. Dynamical evolution of polaron to bipolaron in conjugated polymers. Phys. Rev. B. 2006, 74, 224304-6.

(25) Stafström, S. Electron localization and the transition from adiabatic to nonadiabatic charge transport in organic conductors. Chem. Soc. Rev. 2010, 39, 2484-2499.

(26) Sun, Z.; Stafström, S. Bipolaron recombination in conjugated polymers. J. Chem. Phys. 2011, 135, 074902-7. 


\section{APPENDIX \\ PAPER VII}

Singlet-Singlet Exciton Recombination:

Theoretical Insight into the Influence of High Density Regime of Excitons in

Conjugated Polymers

Luiz Antonio Ribeiro Junior

Wiliam Ferreira da Cunha

Geraldo Magela e Silva

J. Phys. Chem. B., 118, 5250-5257 (2014) 


\title{
Singlet-Singlet Exciton Recombination: Theoretical Insight into the Influence of High Density Regime of Excitons in Conjugated Polymers
}

\author{
Luiz Antonio Ribeiro Junior,* Wiliam Ferreira da Cunha, and Geraldo Magela e Silva* \\ Institute of Physics, Universidade de Brasília, 70.919-970 Brasilia, Brazil
}

ABSTRACT: Using a modified version of the Su-SchriefferHeeger (SSH) model combined with the extended Hubbard model (EHB), the recombination between a singlet exciton pair is investigated under the influence of an external electric field, electron-electron interactions, and temperature effects in the scope of a nonadiabatic evolution method. The excitons are positioned very close to each other in a way to mimic a high-density region in monomolecular conjugated polymer systems. Results show that there are mainly three possible channels resulting from singlet-singlet exciton recombination: (1) forming an excited negative polaron and an excited positive bipolaron, (2) forming two free and excited oppositely charged polarons, and (3) forming a biexciton. These results

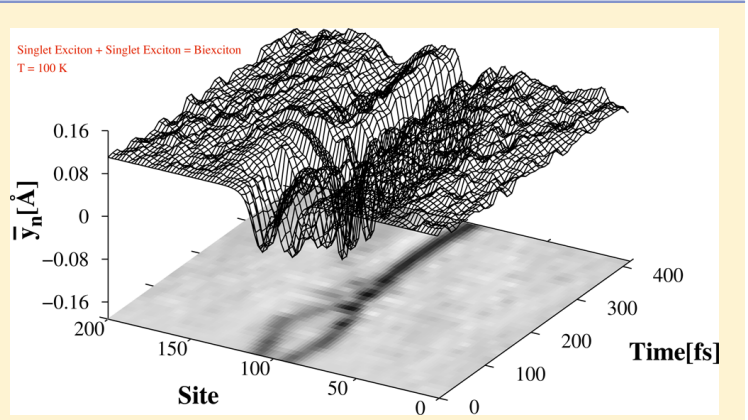
suggest that the recombination processes critically depends on the condition imposed to the system. The description of this dependence, as carried out in the present work, may provide guidance to improve the generation of free charge carriers in organic optoelectronic devices.

\section{INTRODUCTION}

Conjugated polymers have attracted considerable interest because the discovery of electroluminescence properties on phenyl-based organic semiconductors in the 90s. Their optoelectronic features combined with processing advantages, mechanical properties, and low cost make them attractive materials for the electronics industry to provide a promising new display technology. There are several potential applications such as organic photovoltaics devices (OPVs) ${ }^{1-3}$ and polymer light emitting diodes (PLEDs). ${ }^{4-6}$ In contrast to the conventional inorganic conductors, the fact that conjugated polymers are quasi-one-dimensional materials leads to the novel property of its lattice structure being easily distorted to form self-trapped elementary excitations. This can be accomplished either by charge injections or by a photoexcitation mechanism and results in the induction of self-localized electron states, such as an exciton. ${ }^{7,8}$ In conjugate polymers, an exciton is a bound electron-hole pair state formed due to the strong electronlattice interactions. ${ }^{9}$ It is well-known that a large number of excitons are accumulated in working OPVs (forming a highdensity excitons region) and have a significant probability of interacting and recombining. Also, the recombination of the excitons can occurs with other particles, for example, polarons and bipolarons. ${ }^{10-12}$

Among these excitations, the polaron and bipolaron acts on the system as charge carriers, whereas singlet exciton, the excited polaron, and biexciton are light emissive species. ${ }^{13-15}$ It is suggested that the singlet-singlet exciton recombination possibly forms the above-mentioned particles and affects dramatically the working characteristics of OPVs. Although some possible mechanisms for recombination processes between excitons and charge carriers that considered electron-electron interactions and an external electric field have been proposed, no detailed theoretical investigations of how two singlet excitons recombine, when these effects are taken into account, have been reported. Furthermore, it has been generally accepted that temperature effects are one of fundamental importance on monomolecular recombination of mixed states composed of polarons and excitons. ${ }^{16,17}$ Thus, the singlet-singlet exciton recombination in the presence of thermal effects is believed to be essential for OPVs devices. Understanding how the aforementioned effects acts on the monolayer exciton recombination process, which leads to free charge carriers generation, is crucial for the design of more efficient devices and requires a deeper phenomenological description.

In this paper, a systematic numerical investigation considering the influence of an external electric field, Coulomb interactions, and temperature effects on the singlet-singlet exciton recombination process is performed in a monolayer conjugated polymer system in terms of a nonadiabatic evolution method. The exciton pair recombination is investigated by considering a region in the conjugated polymer lattice with high-density of excitons. To mimic the high-density

Received: November 1, 2013

Revised: April 22, 2014

Published: April 22, 2014 
region, the excitons are positioned very close to each other. An Ehrenfest molecular dynamics is performed by using a onedimensional tight-binding model including lattice relaxation. Combined with the EHM, an extended version of the SSH model is used to include external electric fields and Brazoviskii-Kirova symmetry breaking terms. Temperature effects are included by means of a canonical Langevin equation. The aim of this work is to give a physical picture of the products and their yields due to the recombination of a singlet-exciton pair in conjugated polymers, when electronelectron interactions, external electric field, and temperature effects are taken into account. The understanding of these important processes may provide guidance for improving the efficiency of free charge carriers generation in organic optoelectronic deveices.

\section{MODEL AND METHOD}

A conjugated polymer chain is used to study the influence temeperature effects on the generation os excited states. The Hamiltonian of the model is given by $H=H_{\text {elec }}+H_{\text {latt }}+$ $H_{\mathrm{e}-\mathrm{e}}{ }^{18,19}$ The first term in eq 1 is the electronic part of the Hamiltonian modified to include an external electric field and the Brazovskii-Kirova symmetry-breaking and has the following form

$$
H_{\text {elec }}=-\sum_{n, s}\left(t_{n, n+1} C_{n+1, s}^{\dagger} C_{n, s}+\text { hc }\right)
$$

were $n$ indexes the sites of the chain. The operator $C_{n, s}^{\dagger}\left(C_{n, s}\right)$ creates (annihilates) a $\pi$-electron state at the $n$th site with spin $s ; K$ is the harmonic constant that describes a $\sigma$ bond and $M$ is the mass of a $\mathrm{CH}$ group. The parameter $y_{n}$ is defined as $y_{n} \equiv$ $u_{n+1}-u_{n}$ where $u_{n}$ is the lattice displacement of an atom at the $n$th site. $p_{n}$ is the conjugated momentum to $u_{n}$ and $t_{n, n+1}$ is the hopping integral, ${ }^{20}$ given by $t_{n, n+1}=\mathrm{e}^{-\mathrm{i} \gamma \mathrm{A} t}\left[\left(1+(-1)^{n} \delta_{0}\right) t_{0}-\right.$ $\left.\alpha y_{n}\right]$, where $t_{0}$ is the hopping integral of a $\pi$-electron between nearest neighbor sites in the undimerized chain, $\alpha$ is the electron-phonon coupling, and $\delta_{0}$ is the Brazovskii-Kirova symmetry-breaking term, which is used to take the cissymmetry of the polymer into account. $\gamma \equiv e a /(\hbar c)$, with $e$ being the absolute value of the electronic charge, $a$ the lattice constant, and $c$ the speed of light. The relation between the time-dependent vector potential $\mathbf{A}$ and the uniform electric field $\mathbf{E}$ is given by $\mathbf{E}=-(1 / c) \dot{\mathbf{A}}^{15}$ The second term in eq 1 is the Hamiltonian of the lattice backbone, which is treated classically:

$$
H_{\text {latt }}=\sum_{n} \frac{K}{2} y_{n}^{2}+\sum_{n} \frac{p_{n}^{2}}{2 M}
$$

where $K$ is the harmonic constant that describes a $\sigma$ bond and $M$ is the mass of a $\mathrm{CH}$ group. The parameter $y_{n}$ is defined as $y_{n}$ $=u_{n+1}-u_{n}$ and $p_{n}$ is the momentum conjugated to $u_{n}$.

The last contribution denotes $\mathrm{e}-\mathrm{e}$ interactions and can be written as

$$
\begin{aligned}
H_{\mathrm{ee}}= & U \sum_{i}\left(C_{i, \uparrow}^{\dagger} C_{i, \uparrow}-\frac{1}{2}\right)\left(C_{i, \downarrow}^{\dagger} C_{i, \downarrow}-\frac{1}{2}\right) \\
& +V \sum_{i}\left(n_{i}-1\right)\left(n_{i+1}-1\right)
\end{aligned}
$$

where $U$ and $V$ are the on-site and nearest-neighbor Coulomb repulsion strengths, respectively, and $n_{i}=C_{i, \uparrow}^{\dagger} C_{i, \uparrow}+C_{i, \downarrow}^{\dagger} C_{i, \downarrow}{ }^{21} \mathrm{It}$ should be noted that the inclusion of the additional constant factors (related to the conventional description of the Hubbard model) is necessary to maintain the electron hole symmetry of the Hamiltonian. Several theoretical and experimental results have demonstrated that the primary excitation is the exciton, and electron-electron interactions are dominant over electron-lattice interactions in luminescent polymers. ${ }^{22-27}$ Some theoretical studies that have considered the electronlattice interactions also show that introduction of the lattice relaxation effect would not lead to an increase in binding energy of the exciton. ${ }^{28,29}$ These facts enable us to handle electronelectron interactions in long chains and arrive at an understanding of electronic states in luminescent polymers without loss of essential physics. The parameters used here are $t_{0}=2.5 \mathrm{eV}, M=1349.14 \mathrm{eV} \times \mathrm{fs}^{2} / \AA^{2}, K=21 \mathrm{eV} \AA^{-2}, \delta_{0}=0.05$, $\alpha=4.1 \mathrm{eV} \AA^{-1}, a=1.22 \AA$, and a bare optical phonon energy $\hbar \omega_{Q}=\hbar(4 K / M)^{(1 / 2)}=0.16 \mathrm{eV}$. These values have been used in previous simulations and are expected to be valid for conjugated polymers in general. ${ }^{30-36}$

To solve these equations numerically, first a stationary state that is self-consistent with all degrees of freedom of the system (the lattice plus electrons) is obtained. We begin by constructing the Hamiltonian from an arbitrary $\left\{y_{n}\right\}$ set of positions. By solving the time dependent Scrhödinger equation, we obtain a new set of coordinates $\left\{y_{n}^{\prime}\right\}$. Iterative repetitions of this procedure yields a self-consistent initial state when $\left\{y_{n}\right\}$ is close enough to the solution. The equation of motion that describes the site displacement and provides the temporal evolution of the lattice is obtained by a classical approach. The nuclear dynamics is made with the Euler-Lagrange equations

$$
\frac{\mathrm{d}}{\mathrm{d} t}\left(\frac{\partial\langle L\rangle}{\partial \dot{u}_{n}}\right)-\frac{\partial\langle L\rangle}{\partial u_{n}}=0
$$

where $\langle L\rangle=\langle T\rangle-\langle V\rangle$. Equation 4 leads to an Newtonian equation $M \ddot{u}_{n}=F_{n}(t)$. Thus,

$$
\begin{aligned}
F_{n}(t)= & M \ddot{u}_{n}=-K\left[2 u_{n}(t)-u_{n+1}(t)-u_{n-1}(t)\right] \\
& +\alpha\left[B_{n, n+1}-B_{n-1, n}+B_{n+1, n}-B_{n, n-1}\right]
\end{aligned}
$$

where $F_{n}(t)$ represents the force on the $n$th site. ${ }^{37}$ Here, $B_{n, n^{\prime}}=$ $\sum_{k, s}^{\prime} \psi_{k, s}^{*}(n, t) \psi_{k, s}\left(n^{\prime}, t\right)$ is the term that couples the electronic and lattice solutions. The primed summation represents a sum over the occupied states.

The total time dependent wave function was constructed by means of a combination of instantaneous eigenstates of the electronic Hamiltonian. The solutions of the time-dependent Scrhödinger equation can be put in the form

$$
\begin{aligned}
\psi_{k, s}\left(n, t_{j+1}\right)= & \sum_{l}\left[\sum_{m} \varphi_{l, s}^{*}\left(m, t_{j}\right) \psi_{k, s}\left(m, t_{j}\right)\right] \\
& \times \mathrm{e}^{\left(-\mathrm{i} \varepsilon_{l} \Delta t / \hbar\right)} \varphi_{l, s}\left(n, t_{j}\right)
\end{aligned}
$$

$\left\{\phi_{l}(n)\right\}$ and $\left\{\varepsilon_{l}\right\}$ are the eigenfunctions and the eigenvalues of the electronic part for the Hamiltonian at a given time $t_{j}{ }^{38}$ Equation 5, which governs the evolution of system, may be numerically integrated using the method $u_{n}\left(t_{j+1}\right)=u_{n}\left(t_{j}\right)+$ $\dot{u}_{n}\left(t_{j}\right) \Delta t$ and $\dot{u}_{n}\left(t_{j+1}\right)=\dot{u}_{n}\left(t_{j}\right)+\left(F_{n}\left(t_{j}\right) / M\right) \Delta t$. Hence, the electronic wave functions and the lattice displacements at the $(j$ $+1)$ th time step are obtained from the $j$ th time step. At time $t_{j}$ the wave functions $\left\{\psi_{k, s}\left(i, t_{j}\right)\right\}$ can be expressed as a series expansion of the eigenfunctions $\left\{\phi_{l, s}\right\}$ at that moment: $\psi_{k, s}\left(i, t_{j}\right)$ $=\sum_{l=1}^{N} C_{l, k}^{S} \phi_{l, s}(i)$, where $C_{l, k}^{S}$ are the expansion coefficients. The occupation number for eigenstate $\phi_{l, s}$ is $\eta_{l, s}\left(t_{j}\right)=\sum_{k}^{\prime}\left|C_{l, k}^{S}\left(t_{j}\right)\right|^{2}$. 

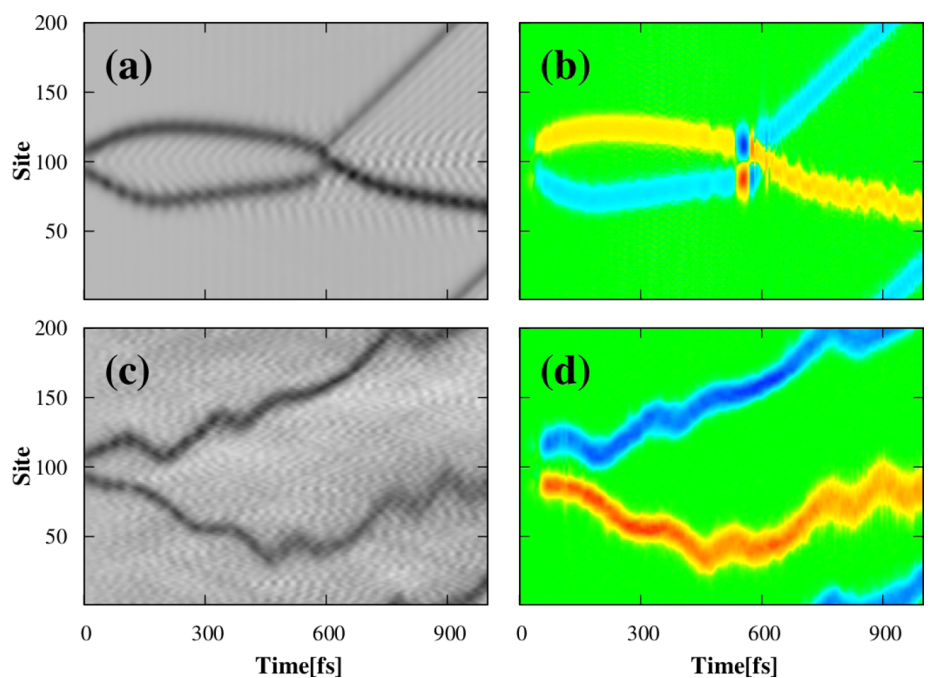

Figure 1. (a) Bond-length time evolution and (b) charge density time evolution for the channel 1 where $E=1.5 \mathrm{mV} / \AA, U=0.3 \mathrm{eV}$, and $T=0 \mathrm{~K}$. (c) Bond-length time evolution and (d) charge density time evolution for the channel 2 using $E=1.5 \mathrm{mV} / \AA, U=0.2 \mathrm{eV}$, and $T=150 \mathrm{~K}$.

$\eta_{l, s}\left(t_{j}\right)$ contains information concerning the redistribution of electrons among the energy levels. ${ }^{39,40}$ Because our model assumes a classical treatment for the lattice, it is possible to use the Langevin approach to consider the thermal effects. It is settled a white stochastic signal $\zeta(t)$ as the fluctuation term, i.e., a signal with the following properties: $\langle\zeta(t) \equiv 0\rangle$ and $\langle\zeta(t)$ $\left.\zeta\left(t^{\prime}\right)=\Lambda \delta\left(t-t^{\prime}\right)\right\rangle$. A Stokes-like dissipation term is also included in this formalism. Therefore, eq 5 is modified to $M \ddot{u}=$ $-\gamma \dot{u}+\zeta(t)+F_{n}(t)$. The relationship between $\zeta, \gamma$, and the temperature $T$ is given the fluctuation-dissipation theorem, $\Lambda$ $=2 k_{\mathrm{B}} T \gamma M$. It is important advise that this approach of considering thermal effects in conjugated polymers has ben used before in the literature and has good track record. $16,17,41-45$

\section{RESULTS AND DISCUSSION}

A systematic numerical investigation considering the influence of external electric field, Coulomb interactions, and temperature effects over the singlet-singlet exciton recombination dynamics is performed in systems composed of 200-site cispolyacetylene with periodic boundary conditions. For the electric field, turned on quasi-adiabatically until $50 \mathrm{fs}^{41}$ the values used in the simulations varied from 0.5 to $2.5 \mathrm{mV} / \AA$ with an increment of $0.5 \mathrm{mV} / \AA$, whereas the on-site electronelectron interactions values considered are $0.1,0.2,0.3,0.4$, and $0.5 \mathrm{eV}$. The nearest-neighbor Coulomb repulsion strength was defined using the relation $V=U / 2$. As already mentioned, the temperature is introduced by means of a Langevin equation to simulate the sites random motion. Thus, the measure of temperature is obtained through the classical equivalence $k_{\mathrm{B}} T=$ $M \dot{u}_{n}{ }^{2}$. It is important to note, however, that special care must be taken on relating a given temperature value to a simulation at different times because the process of thermalization is not carried out instantaneously. In a previous work it was shown that the higher the desired temperature, the more time it takes for the system to relax into this regime. ${ }^{41}$ Because one of the objectives is to perform a qualitative investigation on the temperature influence over the exciton pair recombination, we used $1000 \mathrm{fs}$ for the time of simulation to carry out the analysis and guarantee that the lattice reaches up all the simulated temperature regimes.

Furthermore, the analysis of the quasi-particles dynamics is performed by considering the mean charge density $\bar{\rho}_{n}(t)$, derived from the charge density $\rho_{n}(t)=\sum_{k, s}^{\prime} \psi_{k, s}^{*}(n, t) \psi_{k, s}(n, t)$, and the mean order parameter $\bar{y}_{n}(t)$. The expressions for these quantities are given by $\bar{\rho}(t)=1-\left[\rho_{n-1}(t)+2 \rho_{n}(t)+\rho_{n+1}(t)\right] /$ 4 and $\bar{y}(t)=(-1)^{n}\left[y_{n-1}(t)+2 y_{n}(t)+y_{n+1}(t)\right] / 4$. The goal is to provide a better visualization of the simulations and consequently to perform a more accurate analysis of the results.

In this context, Figure 1 depicts the dynamical process of free charge carrier generation via singlet-singlet exciton recombination. The first channel reported here for the recombination mechanism is showed through the order parameter in Figure 1a and through the charge density time evolution in Figure 1b. For this case, the electric field strength and Coulomb interactions considered are $1.5 \mathrm{mV} / \AA$ and $0.3 \mathrm{eV}$, respectively, in the absence of temperature effects. Initially, the excitons are positioned very close to each other in a way to mimic a highdensity region, as can be seen in the bond-length time evolution shown in Figure 1a. In conjugated polymers, the clearest difference between the triplet and singlet excitons is that the triplet states are more localized than singlet ones. In addition, the distortion at the center of the singlet state is shallower than the triplet state, and the dimerization pattern is also different between them. ${ }^{46}$ A singlet exciton (SE) causes a distortion in the lattice of approximately 40 sites around its center. Considering this fact, to mimic a high-density region, the exciton pair is positioned within 70 sites of the lattice. The presence of such close pair of singlet excitons causes a considerable overlap of their wave functions. The abovementioned differences between singlet and triplet states indicate that the exciton pair recombination process of singlet-singlet or triplet-triplet states may occurs generating different products and yields, even when bimolecular systems are taken into account. ${ }^{42}$ Figure la shows that for the first $50 \mathrm{fs}$, the excitons remain very close to each other, which is an indication of a small interaction between them, consequence of the electron-electron interactions considered. From that 
instant on, the electric field strength becomes considerable and the interaction between the electrons and holes of both excitons is increased, which is easily verified by noting the spacing among the exciton at around $300 \mathrm{fs}$ and the phonons produced through the lattice. These phonons are the direct manifestation of the excitons interaction causing the diffusive pattern presented by Figure 1a. These facts suggest that the electric field is fundamental to increase the interaction between the excitons toward the production of new species. Another important characteristic to observe is that the electric field plays the role of concentrating charge in the quasi-particles lattice distortion. Figure $1 \mathrm{~b}$ presents the charge density time evolution. It is possible to note that before approximately 50 $\mathrm{fs}$, when the electric field regime is too small, there is almost no charge concentration in the system. Immediately after this moment, a negative (blue) and a positive (red) concentration of charge arise in the chain as a consequence of a higher electric field action, in this case, $1.5 \mathrm{mV} / \AA ̊ \AA$. After a small transient time for the electric field response, the generated free charge carriers begin to move toward one another due to the opposite charges they possess. Before the collision between the charge carriers, two excited polarons (polaron-excitons) emerge from the exciton recombination process and their dynamics occurs as described by Stafström and co-workers. ${ }^{33,34}$ At approximately $580 \mathrm{fs}$, when the collision takes place, one can see a transition structure that is momentarily self-trapped around the 100th site for about 200 fs. Thus, after the conditions for creating this transition structure is achieved, the electric field tends to destabilize such structure and to direct the system to the final state composed of a bipolaron-exciton with positive charge and a polaron-exciton that have negative charge. The polaronexciton passes through the bipolaron-exciton in a process that yields the formation of a mixed state composed of charge carriers and excitons after the scattering. Also, the interaction between the hole-bipolaron $\left(\mathrm{BP}^{+}\right)$and the electron-polaron $\left(\mathrm{P}^{-}\right)$increases the production of phonons after the scattering, as showed in Figure 1a. For the simulations performed considering the electric field effects and Coulomb interactions, in the absence of the temperature, the results showed that for all electric field strengths smaller than $1.5 \mathrm{mV} / \AA$ the exciton recombination does not take place. In theses cases, only a separation between the excitons can be observed, in the same fashion as observed in Figure 1a. On the other hand, for electric field regimes greater than this critical value, the same products shown in Figure $1 \mathrm{~b}$ are formed. A further increase in the electric field strength leads to a yield gain in the bipolaron and polaron formation, due to the fact that a larger electrons fraction is transferred among the energy levels of the excitons. This process provides, for example, a well-defined bipolaron with a faster response to the electric field action than the case for the critical electric field showed in Figure la,b.

A more realistic theoretical description is obtained by discussing the qualitative dynamical features of the recombination process between the singlet exciton pair considering the contribution of the temperature effects to this mechanism. Figure 1c,d depicted the second channel for the singlet-singlet exciton recombination reported in this work. For this case, the temperature regime is $150 \mathrm{~K}$, the electric field strength is 1.5 $\mathrm{mV} / \AA$, and the Coulomb interaction is $0.2 \mathrm{eV}$. Figure $1 \mathrm{c}$ shows that, after about $50 \mathrm{fs}$, the adding up of thermal energy begins to become visible through the blurring of the figure. After about $90 \mathrm{fs}$, the lattice oscillations are of such amplitude that causes the direct generation of two free oppositely charged polaron- excitons. In this case, both particle maintain their integrity until the end of the simulation, as can be seen in Figure 1d. However, the disordered pattern on the order parameter, shown in Figure 1c, indicates that a reduction of stability of the polaron-excitons occurs caused by the large amplitude of the lattice vibration induced by the temperature. By analyzing the polaron-excitons trajectory in Figure 1c, one can readily note the influence temperature has over the quasi-particles by the damping of their movements. This behavior is attributed to the fact that the lattice vibration disturbs the system in such a level that the electric field is no longer able to give considerable velocity for the free charge carriers. Also, it was observed that, when the temperature effects are considered in the presence of electron-electron interactions and, most important, of an external electric field, the singlet-singlet exciton recombination in a high-density region leads directly to the formation of identical free charge carriers with opposite charges, i.e., an electron-polaron $\left(\mathrm{P}^{-}\right)$and a hole-polaron $\left(\mathrm{P}^{+}\right)$for all temperature regimes simulated. As discussed in the first channel, for the physical picture presented in Figure 1c,d (second channel), the electric field effects also play to role to produce free charge carriers with better yield than neutral excitations, although the final product is still a mixed state of free charge carriers and neutral excitons. In summary, the second channel depicts the most remarkable result from the simulations reported in this paper, suggesting that there may be an alternative route for creating an oppositely charged polaron pair when a reasonable relation between the density of excitons, electric field, and temperature is considered.

To elucidate the influence of the temperature and electric field effects on the dynamical process of singlet-singlet exciton recombination, the next natural step is the consideration of a system in which temperature effects and Coulomb interaction are taken into account in the absence of an external electric field. Figure 2 presents the remaining recombination channel

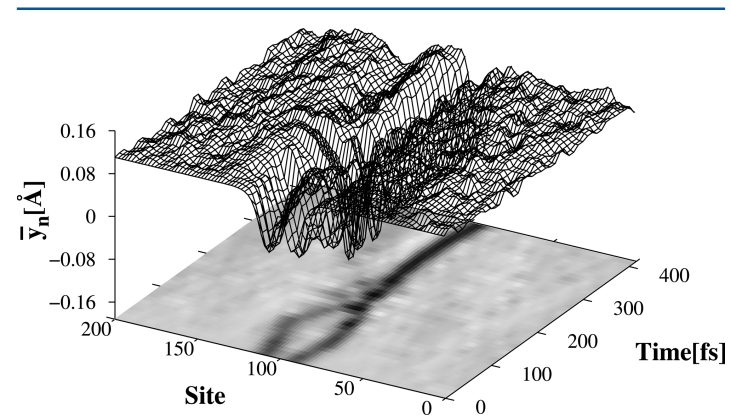

Figure 2. Bond-length order parameter time evolution that depicts the singlet biexcitonic state formation (channel 3) for $T=100 \mathrm{~K}, U=0.2$ $\mathrm{eV}$, and $E=0$.

reported in this work. The figure shows the bond length time evolution for a temperature regime of $100 \mathrm{~K}$ and electronelectron interactions strength of $0.2 \mathrm{eV}$. In this case, the wave functions of the two singlet excitons start to overlap strongly because the two excitons are positioned very close to each other at the beginning of the simulation. The random walk dynamics resultant from temperature fluctuations eventually causes the pair of excitons to be again close together at about $100 \mathrm{fs}$. Together with the temperature effects, the Coulomb interaction of the self-trapped state helps producing a singlet biexciton 
(SBE) deformation by transferring a fraction of the two electrons from a singlet exciton to the other one. The result of this process can be clearly seen observing the lattice deformation before and after the exciton pair recombination in Figure 2. The surface shows the motion of the lattice carbon sites resulting from the thermal oscillations that increases over time, which indicates that the thermalization process is not instantaneous. After $100 \mathrm{fs}$, it is possible to note that only one quasi-particle structure, which characterizes the singlet biexcitonnic state, remains in the lattice. This behavior was observed for all temperature regimes simulated here. Also, by observing the surface projection, shown at the bottom of Figure 2 , one can see that the singlet biexcitonnic structure causes a greater lattice deformation than the conventional singlet exciton. This fact suggests that the biexciton is a more stable structure than the singlet exciton. Indeed, theoretical calculations have shown that the radiative decay process of a biexciton has a large transition dipole moments ( 8.8 e $\AA$ ), in such a way that the biexciton state can decay into a singlet exciton sate by emitting one photon. ${ }^{13}$ Furthermore, the resultant singlet exciton has also a large transition dipole momentum (5.5 e $\AA$ ), which is of the same order of magnitude as that of the biexciton and the singlet exciton decay to the ground state emitting one photon. These results indicate that the above-discussed mechanism of Figure 3, leading to the

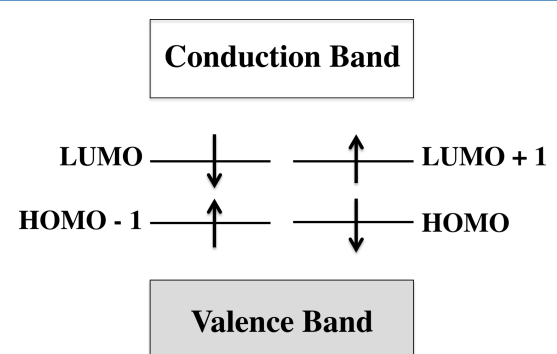

Figure 3. Illustration of the bandgap structure for a polymer chain containing a singlet exciton pair with antiparallel spin configuration.

biexciton formation, requires a more energetic situation than the initial configuration with a singlet-exciton pair for dissociating the biexciton. Also, this process limits the free charge carrier generation mechanism only in the biexciton dissociation at the donor-acceptor heterojunction interface, unlike the results reported in channel 2 . Thus, it is possible to conclude that the temperature effects act on the system in a way to decrease the free charge carrier generation when a region with high-density of excitons is considered. On the other hand, the results reported in channels 1 and 2 indicate that the electric field effects are of fundamental importance and favors the free charge carrier formation.

The yields of the mixed state composed of free charge carriers and neutral excitations, is efficiently described by means of the occupation number time evolution. In this context, Figure 3 shows the schematic diagram of energy levels for a polymer chain containing a singlet exciton pair with antiparallel spin configuration. There are four levels inside the bandgap. The two left levels, HOMO-1 and LUMO, represent the electronic configuration for one of the singlet excitons present in the lattice, in which the upper and the lower ones are occupied by one electron with opposite spins. The right levels,
$\mathrm{HOMO}$ and $\mathrm{LUMO}+1$, represent an alternative route to create a singlet exciton structure, having antiparallel spin configuration with respect to the first one. Figure 4 shows the occupation number time evolution for channel 1 (Figure 4a), channel 2 (Figure 4b), and channel 3 (Figure 4c). In Figure 4a, after the transient period in which the occupation number oscillates due to the strong overlapping of singlet excitons, it is possible to
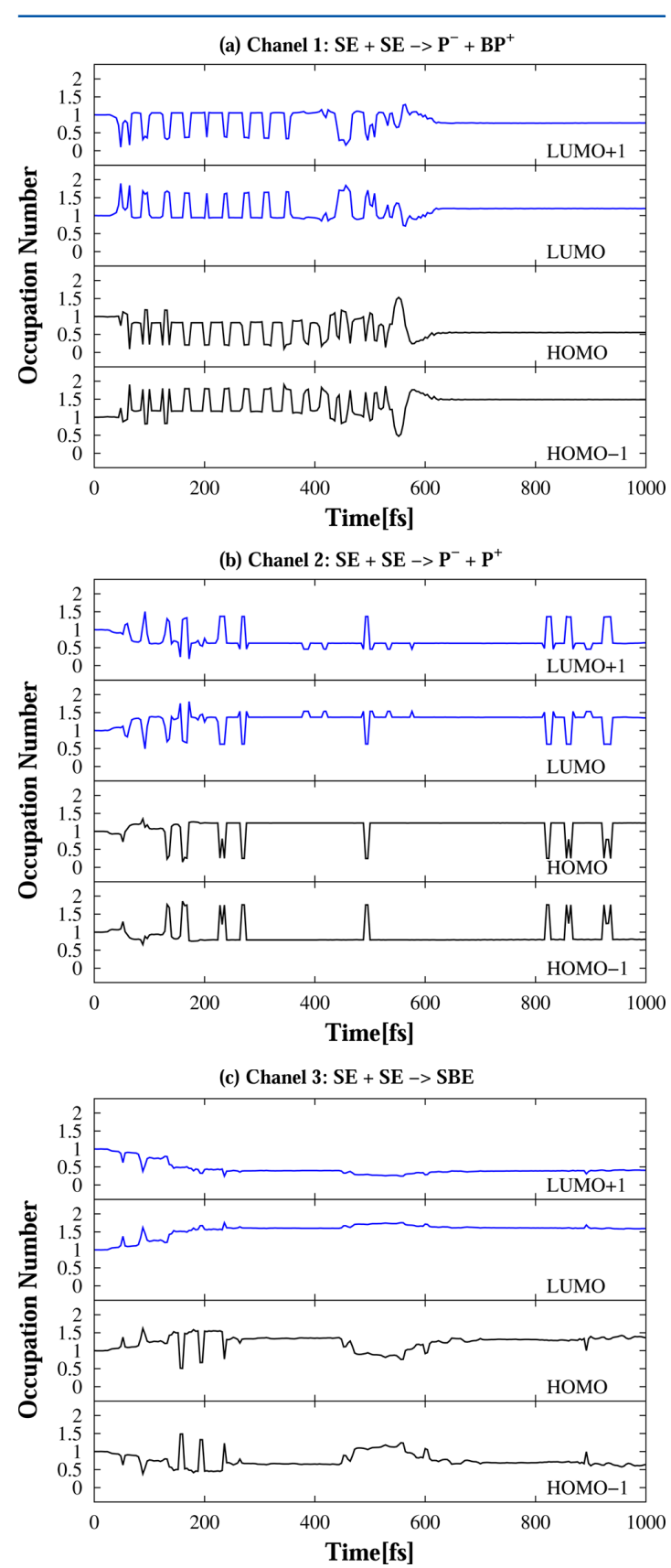

Figure 4. Occupation number time evolution for (a) channel 1, (b) channel 2 , and (c) channel 3. 
note a considerable degree of symmetry between HOMO -1 and HOMO and also between LUMO and LUMO+1, which is an indication of an electron exchange between these levels. The electron exchange after approximately $580 \mathrm{fs}$ between the levels represents the formation of a polaron-exciton structure (electron-polaron + singlet-exciton), being represented by the HOMO-1 level filled almost with two electrons and the LUMO that has one entire electron together with a quite small fraction of another electron. The bipolaron-exciton (holebipolaron + singlet-exciton) has the bandgap electronic configuration presented by the HOMO and LUMO+1 both occupied practically with one electron.

Figure $4 \mathrm{~b}$ shows the occupation number time evolution for the case depicted in the channel 2, where two free polaronexcitons with opposite charge are created. By analyzing the occupation number itself, one can see that the final states are not covered by integer numbers, which is evidence of partial transfer. Moreover, in this case, the occupation numbers do not present the oscillating pattern with high frequency as shown Figure 4a. This fact can be attributed to the temperature influence on the system that gives random motion to the excitons decreasing significantly the overlap interaction between them. The HOMO-1 and the LUMO levels represent the negative polaron-exciton structure (electron-polaron + singlet exciton), whereas the HOMO and the LUMO+1 levels define the positive polaron-exciton (hole-polaron + singlet exciton). In this channel the polaron-excitons formed can be distinguished by observing the LUMO levels. The LUMO+1 level is occupied by a fraction of approximately 0.5 electron, forming the hole-polaron. On the other hand, the LUMO level is filled by 1.5 fraction of electrons, which generates the electron-polaron. Finally, Figure $4 c$ depicts the occupation number time evolution for the physical picture where a biexciton is formed. Through this figure one can see that the final stage of the biexciton formation takes place at approximately $200 \mathrm{fs}$. At the end of the simulation, both levels are occupied by about half electron. Consequently, the HOMO and LUMO levels remain inside the energy gap and are filled by almost 1.5 electrons. Also, in this case the temperature acts on the system in a way to decrease the electron oscillation between the quasi-particle energy levels inside the bandgap.

The analysis of the time evolution of energy levels is recognized as one of the most suitable tools to describe the products generated after a recombination process between quasi-particles in conjugated polymers. ${ }^{11,12,39,40}$ Figure 5 shows the energy level time evolution profile of the simulations that corresponds to the aforementioned channels: Figure 5a shows the energy level behavior for the channel $1\left(\mathrm{SE}+\mathrm{SE} \rightarrow \mathrm{BP}^{+}+\right.$ $\left.\mathrm{P}^{-}\right)$, Figure $5 \mathrm{~b}$ presents the results for the channel $2(\mathrm{SE}+\mathrm{SE}$ $\rightarrow \mathrm{P}^{+}+\mathrm{P}^{-}$), and Figure $5 \mathrm{c}$ for the last discussed channel ( $\mathrm{SE}+$ $\mathrm{SE} \rightarrow \mathrm{SBE}$ ). Concerning these three figures, the first result to consider is that the presence of an external electric is responsible for causing the degeneracy break in the energy levels inside the valence and conduction bands. This conclusion results from the fact that this degeneracy breaking is missing in Figure 5c, which was carried out in the absence of an external electric field. Studying Figure 5a shows the recombination process takes place at approximately $600 \mathrm{fs}$, forming two different new species when the resulting phonons are associated with the oscillation of the energy levels inside the bandgap. The signature of the hole-bipolaron $\left(\mathrm{BP}^{+}\right)$formation is related to the presence of states deep inside the energy gap (blue lines) that represents its integrity. The red levels, which remain
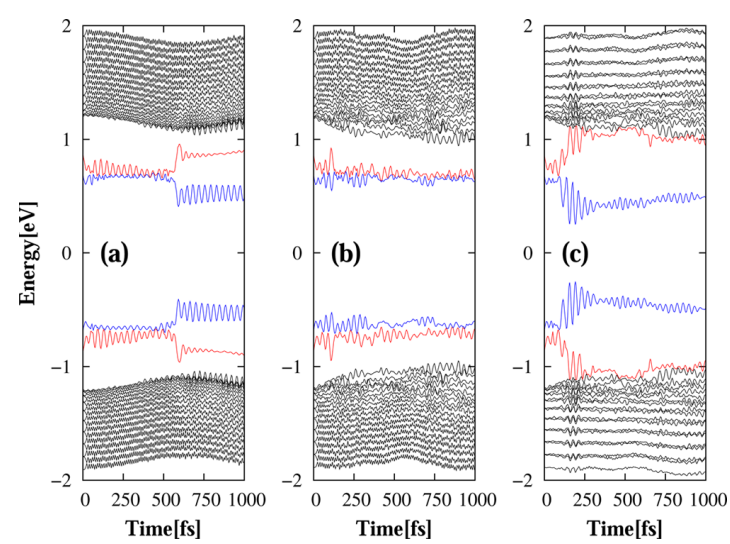

Figure 5. Energy levels time evolution for the (a) channel 1, SE + SE $\rightarrow \mathrm{BP}^{+}+\mathrm{P}^{-},(\mathrm{b})$ channel $2, \mathrm{SE}+\mathrm{SE} \rightarrow \mathrm{P}^{+}+\mathrm{P}^{-}$, and (c) channel 3, $\mathrm{SE}$ $+\mathrm{SE} \rightarrow \mathrm{SBE}$.

consistently inside the bandgap, represent the electron-polaron $\left(\mathrm{P}^{-}\right)$formation and are closer of the valence and conduction band. In Figure $5 \mathrm{~b}$ it is possible observe that the two oppositely charged polaron-excitons are formed directly after approximately $50 \mathrm{fs}$. Also, comparing the polaron-exciton energy levels inside the bandgap of Figure $5 \mathrm{~b}$ with the red levels (that represents the polaron-exciton) in Figure 5a, one can see that the red levels in Figure 5a are closer to the valence and conduction band than all the other levels present inside the bandgap of Figure $5 \mathrm{~b}$. This fact suggests that the polaronexciton formed in the recombination process described for channel 1 has a yield of polaron formation greater than excitons. On the other hand, for the recombination products reported in channel 2, the energy levels behavior indicates that the mixed state composed of polarons and neutral excitations formed after the recombination process present a higher yield in the excitons formation. Once the simulations presented in the channel 1 were performed in the absence of the temperature, the results reported in channels 2 and 3 are evidence that the thermal effects play the role of producing neutral excitations after the exciton recombination process. The singlet biexcitonic state generation of Figure 2 corresponds to features that can be exactly inferred by analyzing the red energy levels that returns to the valence and conduction band, as shown in Figure 5c, thus leading to the vanishing of one singlet exciton. Furthermore, in Figure 5c, at about $100 \mathrm{fs}$, it is possible to note that the recombination takes place and blue levels, which represent the singlet biexciton, remain inside the energy gap until the end of the simulation. It is important to remark that temperature effects increase the oscillation pattern presented by the energy levels due to the presence of phonons with higher energies. However, these effects do not act in a way to break the degeneracy in the levels inside the valence and conduction band.

\section{SUMMARY AND CONCLUSIONS}

A modified version of the SSH model to include an external electric field, the Brazovskii-Kirova symmetry breaking term, one-site and nearest-neighbor electron-electron Coulomb interactions, and temperature is presented to investigate the effects of these properties over the recombination process of a singlet exciton pair in cis-symmetry conducting polymers 
chains. Using a nonadiabatic evolution method, within a onedimensional tight-binding model, the excitons are positioned very close to each other in a way to mimic a high-density region in monomolecular systems. Considering this physical picture, one finds that there are three possible channels resulting from singlet-singlet exciton recombination: (1) formation of an excited negative polaron and an excited positive bipolaron, in the absence of temperature, when the effects over the system of an external electric field and Coulomb interaction are taken into account; (2) generation of two free and excited oppositely charged polarons, when the thermal effects are considered together with an moderate external electric field regime and electron-electron interactions; (3) creation of a biexciton in the absence of an external electric field. These results suggest that temperature effects act on the system in a way to decrease the free charge carrier formation when a region with highdensity of excitons is considered, whereas electric field effects are of fundamental importance and favors the free charge carrier generation. Moreover, the second channel represents the principal result from the simulations reported in this work, which suggests that there may be an alternative route to creating free charge carriers (hole-polaron and electronpolaron) when a appropriate relation between the density of excitons, electric field, and temperature is considered.

\section{AUTHOR INFORMATION}

\section{Corresponding Authors}

*L. A. Ribeiro Junior: e-mail, ribeirojr@fis.unb.br.

*G. M. e Silva: e-mail, magela@fis.unb.br.

Notes

The authors declare no competing financial interest.

\section{ACKNOWLEDGMENTS}

The gratefully acknowledge the financial support from the Brazilian Research Councils CAPES, CNPq, and FINATEC.

\section{REFERENCES}

(1) Menke, S. M.; Luhman, W. A.; Holmes, R. J. Tailored Exciton Diffusion in Organic Photovoltaic Cells for Enhanced Power Conversion Efficiency. Nat. Mater. 2013, 12, 152-157.

(2) Jailaubekov, A. E.; Willard, A. P.; Tritsch, J. R.; Chan, W.-L.; Sai, N.; Gearba, R.; Kaake, L. G.; Williams, K. J.; Leung, K.; Rossk, P. J.; Zhu, X.-Y. Hot Charge-Transfer Excitons Set the Time Limit for Charge Separation at Donor/Acceptor interfaces in organic photovoltaics. Nat. Mater. 2013, 12, 66-73.

(3) Grancini, G.; Maiuri, M.; Fazzi, D.; Petrozza, A.; Egelhaaf, H.-J.; Brida, D.; Cerullo, G.; Lanzani, G. Hot Exciton Dissociation in Polymer Solar Cells. Nat. Mater. 2013, 12, 29-33.

(4) Yang, Q.; Liu, Y.; Pan, C.; Chen, J.; Wen, X.; Wang, Z. L. Largely Enhanced Efficiency in ZnO Nanowire/p-Polymer Hybridized Inorganic/Organic Ultraviolet Light-Emitting Diode by Piezo-Phototronic Effect. Nano Lett. 2013, 13, 607-613.

(5) Tang, S.; Pan, J.; Buchholz, H. A.; Edman, L. White Light from a Single-Emitter Light-Emitting Electrochemical Cell. J. Am. Chem. Soc. 2013, 13, 3647-3652.

(6) Zuniga, C. A.; Abdallah, J.; Haske, W.; Zhang, Y.; Coropceanu, I.; Barlow, S.; Kippelen, B.; Marder, S. R. Crosslinking Using Rapid Thermal Processing for the Fabrication of Efficient Solution-Processed Phosphorescent Organic Light-Emitting Diodes. Adv. Mater. 2013, 25, 1793-1744.

(7) Barford, W. Excitons in Conjugated Polymers: A Tale of Two Particles. J. Phys. Chem. A 2013, 25, 2665-2671.

(8) Barford, W. Theory of Singlet Exciton Yield in Light-Emitting Polymers. Phys. Rev. B 2004, 70, 205204-205212.
(9) Brazovskii, S.; Kirova, N. Physical Theory of Excitons in Conducting Polymers. Chem. Soc. Rev. 2010, 39, 2453-2465.

(10) Meng, Y.; Liu, X. J.; Di, B.; An, Z. Recombination of Polaron and Exciton in Conjugated Polymers. J. Chem. Phys. 2009, 131, 244502-5.

(11) Sun, Z.; Liu, D.; Stafström, S.; An, Z. Scattering Process Between Polaron and Exciton in Conjugated Polymers. J. Chem. Phys. 2011, 134, 044906-044911.

(12) Sun, Z.; Li, Y.; Li, D. S.; An, Z.; Xie, S. Scattering Process Between Bipolaron and Exciton in Conjugated Polymers. Phys. Rev. B 2009, 134, 201310(R).

(13) Di, B.; Meng, Y.; Wang, Y. D.; Liu, X. J.; An, Z. Formation and Evolution Dynamics of Bipolarons in Conjugated Polymers. J. Chem. Phys. B 2011, 115, 964-971.

(14) Di, B.; Meng, Y.; Wang, Y. D.; Liu, X. J.; An, Z. Electroluminescence Enhancement in Polymer Light-Emitting Diodes through Inelastic Scattering of Oppositely Charged Bipolarons. J. Chem. Phys. B 2011, 115, 9339-9344.

(15) Stafström, S. Electron Localization and the Transition from Adiabatic to Nonadiabatic Charge Transport in Organic Conductors. Chem. Soc. Rev. 2010, 39, 2484-2499.

(16) Ribeiro, L. A.; Neto, P. H. O.; da Cunha, W. F.; Roncaratti, L. F.; Gargano, R.; da Silva Filho, D. A.; e Silva, G. M. Exciton Dissociation and Charge Carrier Recombination Processes in Organic Semiconductors. J. Chem. Phys. 2011, 135, 224901-224906.

(17) Neto, P. H. O.; da Cunha, W. F.; Roncaratti, L. F.; Gargano, R; e Silva, G. M. Thermal Effects on Photogeneration of Free Carriers in Organic Conductors. Chem. Phys. Lett. 2010, 493, 283-287.

(18) Su, W. P.; Schrieffer, J. R.; Heeger, A. J. Solitons in Polyacetylene. Phys. Rev. Lett. 1979, 42, 1698-1701.

(19) Su, W. P.; Schrieffer, J. R.; Heeger, A. J. Solitons Excitations in Polyeacetylene. Phys. Rev. B 1980, 22, 2099-2111.

(20) Pinheiro, C. S.; e Silva, G. M. Use of Polarons and Bipolarons in Logical Switches Based on Conjugated Polymers. Phys. Rev. B 2002, 65, 094304-5.

(21) Yu, Z. G.; Wu, M. W.; Rao, X. S.; Bishop, A. R. Excitons in Two Coupled Conjugated Polymer Chains. J. Phys. Condes. Matter 1996, 8, $8847-8857$.

(22) Leng, J. M.; Jeglinski, S.; Wei, X.; Benner, R. E.; Vardeny, Z. V.; Guo, F.; Mazumdar, S. Optical Probes of Excited States in Poly(pPhenylenevinylene). Phys. Rev. Lett. 1994, 72, 156-159.

(23) Hsu, J. W. P.; Yan, M.; Jedju, T. M.; Rothberg, L. J.; Hsieh, B. R. Assignment of the Picosecond Photoinduced Absorption in Phenylene Vinylene Polymers. Phys. Rev. B 1994, 49, 712(R).

(24) Chandross, M.; Mazumdar, S.; Jeglinski, S.; Wei, X.; Vardeny, Z. V.; Kwock, E. W.; Miller, T. M. Excitons in Poly(para-Phenylenevinylene). Phys. Rev. B 1994, 50, 14702(R).

(25) Yu, Z. G.; Fu, R. T.; Wu, C. Q.; Sun, X.; Nasu, K. Excitons, Biexcitons, and the Band Gap in Poly(P-Phenylenevinylene). Phys. Rev. B 1995, 52, 4849-4854.

(26) Guo, F.; Chandross, M.; Mazumdar, S. Stable Biexcitons in Conjugated Polymers. Phys. Rev. Lett. 1995, 74, 2086-2089.

(27) Soos, Z. G.; Ramasesha, S.; Galvao, D. S.; Etemad, S. Excitation and Relaxation Energies of Trans-Stilbene: Confined Singlet, Triplet, and Charged Bipolarons. Phys. Rev. B 1993, 47, 1742-1753.

(28) Ramasesha, S.; Pati, S. K.; Krishnamurthy, H. R.; Shuai, Z.; Brédas, J. L. Symmetrized Density-Matrix Renormalization-Group Method for Excited States of Hubbard Models. Phys. Rev. B 1996, 54, $7598-7600$

(29) Shuai, Z.; Brédas, J. L.; Pati, S. K.; Ramasesha, S. Exciton Binding Energy in the Strong Correlation Limit of Conjugated Chains. Phys. Rev. B 1998, 58, 15329-15331.

(30) Neto, P. H. O.; da Cunha, W. F.; Teixeira, J. F.; Gargano, R.; e Silva, G. M. Electron-Lattice Coupling in Armchair Graphene Nanoribbons. J. Phys. Chem. Lett. 2012, 3, 3039-3042.

(31) Ribeiro, L. A.; da Cunha, W. F.; Neto, P. H. O.; Gargano, R.; e Silva, G. M. Predicting the Equilibrium Structure of Organic Semiconductors With Genetic Algorithms. Chem. Phys. Lett. 2013, $555,168-172$. 
(32) Ribeiro, L. A.; da Cunha, W. F.; Neto, P. H. O.; e Silva, G. M. Dynamics of Photogenerated Polaron-Excitons in Organic Semiconductors. Phys. Proc. 2012, 28, 112-116.

(33) Johansson, A.; Stafström, S. Polaron Dynamics in a System of Coupled Conjugated Polymer Chains. Phys. Rev. Lett. 2001, 86, 36023605 .

(34) Johansson, A.; Stafström, S. Nonadiabatic Simulations of Polaron Dynamics. Phys. Rev. B 2004, 69, 235205-7.

(35) An, Z.; Wu, C. Q.; Sun, X. Dynamics of Photogenerated Polarons in Conjugated Polymers. Phys. Rev. Lett. 2004, 93, 216407-4.

(36) e Silva, G. M.; Terai, A. Dynamics of Solitons in Polyacetylene With Interchain Coupling. Phys. Rev. B 1993, 47, 12568-12577.

(37) e Silva, G. M. Electric-Field Effects on the Competition Between Polarons and Bipolarons in Conjugated Polymers. Phys. Rev. B 2000, 61, 10777-10781.

(38) Lima, M. P.; e Silva, G. M. Dynamical Evolution of Polaron to Bipolaron in Conjugated Polymers. Phys. Rev. B 2006, 74, 224303-6.

(39) Ribeiro, L. A.; da Cunha, W. F.; Neto, P. H. O.; Gargano, R.; e Silva, G. M. Impurity Effects on Polaron-Exciton Formation in Conjugated Polymers. J. Chem. Phys. 2013, 139, 174903-114.

(40) Ribeiro, L. A.; da Cunha, W. F.; Neto, P. H. O.; Gargano, R.; e Silva, G. M. Dynamical Study of Impurity Effects on BipolaronBipolaron and Bipolaron-Polaron Scattering in Conjugated Polymers. J. Phys. Chem. B 2013, 117, 11801-11811.

(41) Ribeiro, L. A.; da Cunha, W. F.; Neto, P. H. O.; Gargano, R.; e Silva, G. M. Impurity Effects and Temperature Influence on the Exciton Dissociation Dynamics in Conjugated Polymers. Chem. Phys. Lett. 2013, 580, 108-114.

(42) Ribeiro, L. A.; da Cunha, W. F.; Neto, P. H. O.; Gargano, R.; e Silva, G. M. Effects of Temperature and Electric Field Induced Phase Transitions on the Dynamics of Polarons and Bipolarons. New J. Chem. 2013, 37, 2829-2836.

(43) Sun, Z.; Stafstöm, S. Dynamics of Exciton Dissociation in Donor-Acceptor Polymer Heterojunctions. J. Chem. Phys. 2013, 138, 164905-8.

(44) Roncaratti, L. F.; Gargano, R.; de Oliveira Neto, P. H.; da Cunha, W. F.; da Silva Filho, D. A.; e Silva, G. M. TemperatureInduced Oscillating Electric Dipole in Conjugated Systems. Chem. Phys. Lett. 2012, 593, 214-217.

(45) Roncaratti, L. F.; Gargano, R.; e Silva, G. M. Theoretical Temperature Dependence of the Charge-Carrier Mobility in Semiconducting Polymers. J. Phys. Chem. A 2009, 113, 14591-14594.

(46) Sun, Z.; Stafström, S. Spin-Dependent Polaron Recombination in Conjugated Polymers. J. Chem. Phys. 2012, 136, 244901-5. 


\section{PAPER VIII}

APPENDIX $\rfloor$

\section{Temperature Effects on the Scattering of Polarons and Bipolarons in Organic Conductors}

Luiz Antonio Ribeiro Junior Wiliam Ferreira da Cunha

Geraldo Magela e Silva

J. Phys. Chem. A., 118, 6272-6277 (2014) 


\title{
Temperature Effects on the Scattering of Polarons and Bipolarons in Organic Conductors
}

\author{
Luiz Antonio Ribeiro Junior, ${ }^{* \dagger}$ Wiliam Ferreira da Cunha, ${ }^{* \dagger}$ and Geraldo Magela e Silva ${ }^{\ddagger}$ \\ ${ }^{\dagger}$ Department of Physics, Chemistry and Biology (IFM), Linköping University, SE-581 83 Linköping, Sweden \\ ${ }^{\ddagger}$ Institute of Physics, University of Brasilia, 70919-970, Brasilia, Brazil
}

ABSTRACT: The scattering process between an electron-polaron and a holebipolaron has been simulated using a version of the $\mathrm{Su}-\mathrm{Schrieffer}-\mathrm{Heeger}$ (SSH) model modified to include an external electric field, Coulomb interactions, and temperature effects in the scope of nonadiabatic molecular dynamics. The simulations reveal remarkable details concerning the polaron-bipolaron recombination reaction. It is found that there exists a critical temperature regime below which a hole-bipolaron and a mixed state composed by an electron-polaron and an exciton are formed and a hole-bipolaron and a free electron are the resulting products of the collisional process, if the temperature is higher than the critical value. In addition, it is obtained that both channels depend sensitively on the strength of the applied electric field. These significant results may provide guidance to understand processes regarding electroluminescence in polymer diodes.

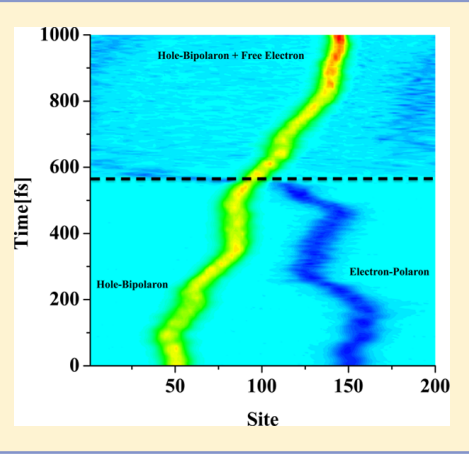

\section{INTRODUCTION}

Conjugated polymers have attracted considerable interest as potential candidates for the development of optoelectronic devices since the discovery of poly(paraphenylene vinylene) (PPV) and its electroluminescence properties in the past few decades. Their optoelectronic features combined with potential advantages in terms of easy synthesis, flexibility, low cost, and large-area capability make them more attractive materials for the electronics industry to provide new display technology than their inorganic counterparts. They have been successfully implemented as the active component in various applications such as organic photovoltaics devices (OPVs) ${ }^{1}$ and polymer light emitting diodes (PLEDs). ${ }^{2}$ In these devices, the generation of excited states is of fundamental physical importance. Particularly, the charge carrier recombination is the key step behind the mechanism of electroluminescence in PLEDs.

Experiments have shown, through optical and magnetic data, that some doped polymers showed signals involving polarons and bipolarons, which proves the coexistence of these charge carriers in conjugated polymers. ${ }^{3,4}$ Thus, there exists a great possibility of collision and recombination between them. A considerable amount of theoretical work focused on understanding the processes underlying the excited states formation in conjugated polymers. The collision mechanism of oppositely charged carriers has also been extensively reported. ${ }^{5-13}$ Nevertheless, theoretical studies that take into account the temperature influence on such mechanisms remain unavailable. It is worthy to mention that, from the experimental point of view, the PPV is one of the best choices for the fabrication of PLEDs, especially for the characteristic of forming thermally stable thin films with high photoluminescence yields. It was also experimentally obtained that the photoexcitation dynamics in
PPV is similar to that of polyacetylene, ${ }^{14}$ which allows the use of polyacetylene in theoretical studies in order to obtain a good physical insight about processes involving charge transport and recombination in real systems. Furthermore, it should be emphasized that the collision between an electron-polaron and a hole-bipolaron was experimentally obtained as a possible channel to produce a new polaronic state in PPV-based light emitting diodes. ${ }^{15}$

Recently, it was experimentally reported that the capture of a positive bipolaron by a deeply trapped negative polaron is one of the mechanism of trion formation in a PPV derivative. ${ }^{16}$ Sun and collaborators investigated theoretically the recombination process between an oppositely charged polaron-bipolaron pair in conjugated polymers using a modified version of the SSH model. ${ }^{17}$ They pointed out that below a critical electric field, the polaron and bipolaron can scatter into an excited polaron with high yield. Above the critical electric field, the polaron and bipolaron will pass through each other and continue moving as isolated structures. By use of a similar approach, the results obtained by Onodera showed that polarons and bipolarons can freely pass through each other without attractive or repulsive interactions. ${ }^{18}$ Theoretical studies carried out by e Silva have shown that the presence of a bipolaron limits the polaron movement in a such way that the polaron cannot cross the bipolaron keeping its integrity after the nonreactive collisional process, resulting in a physical picture where the polaron mobility is compromised. ${ }^{19}$ It is possible that all the results regarding the polaron-bipolaron reaction are not fully comprehensive so that further investigations are needed.

Received: June 5, 2014

Revised: July 21, 2014

Published: July 30, 2014 
Recently, a previous work of ours has also shown that for several electric fields regimes, the presence of impurities in a conjugated polymer lattice favors the excited states formation improving the excitation yields when the scattering between a oppositely charged polaron-bipolaron pair is considered. ${ }^{20}$ In another theoretical work, ${ }^{6}$ it was reported that when a polaron pair enters the coupling area, considering an intrachain scattering process, its velocity slightly increases but the collisional process of two oppositely charged polarons is the same as that in the isolated single polymer chain case, as if the second chain does not exist at all. This means that interchain coupling effect on such mechanism is not important in the cases of intrachain scattering. The same behavior can be expected when the collision process between a polaronbipolaron pair is taken into account. There are, however, still many controversial aspects regarding the influence of some physical process such as temperature, electric field strength, Coulomb interactions, and impurity effects on the formation of excited states via recombination process between oppositely charged carriers, which requires a detailed phenomenological descriptions.

In this paper, a systematic numerical investigation considering the influence of temperature effects, electron-electron interactions, and an external electric field on the collisional process of an oppositely charged polaron-bipolaron pair is performed using a cis-polyacetylene chain. An extended version of the SSH model that includes the Brazovskii-Kirova type symmetry-breaking term is used considering also the extended Hubbard model (EHM). Temperature effects are included by means of a canonical Langevin equation. The results show that the products formed from the polaron-bipolaron reaction, mainly when temperature effects are considered, open a new channel to understanding the polymer luminescence processes and can provide guidance for improving the electroluminescence yields in PLDEs.

\section{MODEL AND METHOD}

The overall SSH-type Hamiltonian ${ }^{21,22}$ modified to include the Brazovskii-Kirova type symmetry-breaking term, ${ }^{23}$ Coulomb interactions, and an external electric field is given by

$$
\begin{aligned}
H_{\text {SSH }}= & -\sum_{n, s}\left(t_{n, n+1} C_{n+1, s}^{\dagger} C_{n, s}+h c\right) \\
& +U \sum_{i}\left(C_{i, \uparrow}^{\dagger} C_{i, \uparrow}-\frac{1}{2}\right)\left(C_{i, \downarrow}^{\dagger} C_{i, \downarrow}-\frac{1}{2}\right) \\
& +V \sum_{i}\left(n_{i}-1\right)\left(n_{i+1}-1\right)+\sum_{n} \frac{K}{2} y_{n}^{2} \\
& +\sum_{n} \frac{p_{n}^{2}}{2 M}
\end{aligned}
$$

where $t_{n, n+1}=\mathrm{e}^{-\mathrm{i} \gamma A}\left[\left(1+(-1)^{n} \delta_{0}\right) t_{0}-\alpha y_{n}\right]$, in which $t_{0}$ is the transfer integral of $\pi$-electrons between nearest neighboring sites in a regular lattice, $\alpha$ is the electron-phonon coupling, $\delta_{0}$ is the Brazovskii-Kirova symmetry-breaking term, and $n$ the site index. $\gamma \equiv e a /(\hbar c)$, where $a$ is the lattice constant, $c$ is the speed of light, and $e$ the absolute value of the electronic charge. $y_{n}$ is defined as $y_{n} \equiv u_{n+1}-u_{n}$, in which $u_{n}$ is the lattice displacement of an atom at the $n$th site. The electric field is included in the Hamiltonian as a scalar potential $A$ having the time dependence in the form of a half Gaussian. ${ }^{24,25}$ The operator $C_{n, s}^{\dagger}\left(C_{n, s}\right)$ creates (annihilates) a $\pi$-electron state at the $n$th site with spin $s$ and $n_{\mathrm{i}}=C_{i, \uparrow}^{\dagger} C_{i, \uparrow}+C_{i, \downarrow}^{\dagger} C_{i, \downarrow} . U$ gives the strength of the on-site and $V$ the nearest-neighbor electronelectron interactions. ${ }^{26} p_{n}$ is the conjugated momentum to $u_{n}$. K is the harmonic constant that describes a $\sigma$ bond, and $M$ is the mass of a $\mathrm{CH}$ group. The parameters used here are those well accepted for a polyacetylene chain: ${ }^{27-33} t_{0}=2.5 \mathrm{eV}, M=$ $1349.14 \mathrm{eV} \cdot \mathrm{fs}^{2} / \AA^{2}, K=21 \mathrm{eV} \AA^{-2}, \delta_{0}=0.05, \alpha=4.1 \mathrm{eV} \AA^{-1}, a$ $=1.22 \AA$, and a bare optical phonon energy $\hbar \omega_{\mathrm{Q}}=\hbar(4 \mathrm{~K} / \mathrm{M})^{1 / 2}$ $=0.16 \mathrm{eV}$.

The initial configuration for the system, i.e., the bond and electronic structures, is obtained by solving the self-consistent equations for the lattice configuration $y_{n}$ and the electronic wave functions. ${ }^{13}$ Once the initial structure was determined, the temporal evolution of the system is carried out by means of the Ehrenfest molecular dynamics, in which the lattice backbone dynamics is performed classically through the Euler-Lagrange equation $\mathrm{s}^{20}$ and the wave functions are obtained by solving the time-dependent Schrödinger according to Ono's method. ${ }^{34}$ The occupation number of the instantaneous eigenstates, as discussed in the next section, is calculated according to ref 35 . Since the approach adopted here treats the lattice backbone classically, it is possible to use the Langevin equation to take into account temperature effects. In this way, a white stochastic signal $\zeta(t)$ is considered as the fluctuation term having the following properties: $\langle\zeta(t)\rangle \equiv 0$ and $\left\langle\zeta(t) \zeta\left(t^{\prime}\right)\right\rangle=\Lambda \delta\left(t-t^{\prime}\right)$. A dissipation term is also included in this formalism in order to keep the temperature constant after a transient period. The relationship between $\zeta, \gamma$, and the temperature $T$ is given by the fluctuation-dissipation theorem, $\Lambda=2 k_{\mathrm{B}} T \gamma M$. It should be emphasized that this methodology to consider temperature effects in one-dimensional conjugated polymer lattice has been used successfully in the literature. ${ }^{25,35-41}$

\section{RESULTS AND DISCUSSION}

Systematic numerical investigations are carried out considering the temperature influence over the collision dynamics of a bipolaron-polaron pair in systems composed of 200-site cispolyacetylene chains. A long polymer chain is necessary to avoid the overlap between the two deformations created after the charge remotion (hole-bipolaron) and injection (electronpolaron). For the electric field, turned on quasi-adiabatically, ${ }^{37}$ the values used in the simulations varied from 0.5 to $2.0 \mathrm{mV} / \AA$ with an increment of $0.5 \mathrm{mV} / \AA$, whereas the temperature regimes considered range from 50 to $250 \mathrm{~K}$ with a step of $25 \mathrm{~K}$.

For the on-site electron-electron interactions the value considered is $U=0.2 t_{0}$. The nearest-neighbor Coulomb repulsion strength was defined using the relation $V=U / 2$. In this context, Figure 1 presents the schematic diagram of energy levels for a lattice containing a hole-bipolaron and an electronpolaron. These charge carriers are formed by removing or adding electrons to the polymer chain, causing lattice distortions and the rising of energy levels inside the band gap. A single polymer chain containing a hole-bipolaron is represented by the absence of electrons in the $(\mathrm{H})$ and $(\mathrm{L})$ levels, which yields a $+2 e$ charge. On the other hand, an electron-polaron is represented by occupied levels $(\mathrm{H}-1)$ and $(L+1)$, in which the upper one is occupied by one electron and the lower one occupied by two electrons, yielding a $-e$ charge. One can see the characteristic larger narrowing of the bipolarons energy levels when compared to those of the polaron, which indicates that the bipolarons are quasi-particles more stable than polarons in conjugated polymers. 


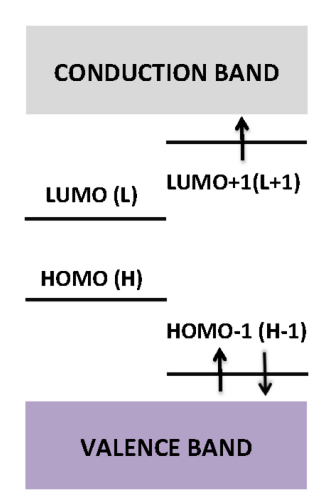

Figure 1. Schematic diagram of energy levels for a polymer chain containing an electron-polaron and a hole-bipolaron.

First, we have investigated the contribution of low temperature regimes in the mechanisms involved in polaronbipolaron scattering. Figure 2 presents the evolution of the mean charge density $\bar{\rho}(t)=1-\left[\rho_{n-1}(t)+2 \rho_{n}(t)+\rho_{n+1}(t)\right] / 4$ at temperatures of $75 \mathrm{~K}$ (Figure $2 \mathrm{a}$ ) and $150 \mathrm{~K}$ (Figure $2 \mathrm{~b}$ ). The positive bipolaron (red structure) is initially located at the 50 th site, while the negative polaron (blue structure) is at 150 th site. The two charge carriers are separated far enough from one another so that they behave as independent structures. Considering an electric field strength of $1.0 \mathrm{mV} / \AA$, after a small transient time for the field response, the charge carriers begin to move toward one another because of the opposite charges. One can readily note the difference between the polaron and the bipolaron dynamics regarding the response of the applied electric field. This is natural if one considers the difference between the masses and charges of these quasiparticles. In this way, the polaron and the bipolaron are accelerated and rapidly reach their saturation velocities. Before the collision, the motion for both charge carriers is the same as that reported in the literature. ${ }^{25}$ Initially, the polaron and the bipolaron move linearly with time. However, after about $100 \mathrm{fs}$ the adding up of thermal energy begins to become considerable and the polaron starts to perform a random walk along the chain influenced by the thermal random forces. Because of its larger effective mass, the bipolaron starts to perform a random walk dynamics only after $300 \mathrm{fs}$. Furthermore, is easy to verify in Figure 2a that the random walk displacement of the polaron is higher than the displacement imposed by the random forces to the bipolaron. As aforementioned, this fact can be understood by considering the charge and mass differences between these charge carriers.

At around $600 \mathrm{fs}$, when the collision takes place, one can see in Figure 2a that the polaron can easily pass through the bipolaron because of its high kinetic energy, a process that yields the formation of a mixed state composed of a polaron and an exciton, while the bipolaron remains intact. The polaron-exciton structure formed is manifested by the charge delocalization pattern presented by the electron-polaron after 700 fs. A small fraction of the electron that occupies the $(L+1)$ level is excited to the conduction band, forming a bond state structure between the electron-polaron and the excited fraction of the electron. The channel reported in Figure 2a (channel 1) is observed in our simulations to occur for temperatures regimes smaller than $75 \mathrm{~K}$ and electric field strengths higher than $1.0 \mathrm{mV} / \AA \AA$. Otherwise, for temperatures higher than this critical value (considering field strengths smaller than $1.0 \mathrm{mV}$ / $\AA$ ) the motion of the charge carriers is damped, as reported in ref 25 , the collision between the charge carriers driven by the external electric field is not observed, and the random walk dynamics dominates.

The role played by the temperature in the collisional process between the charge carriers can be verified considering a temperature regime twice as high as the previous and an electric field strength of $1.5 \mathrm{mV} / \AA$, as shown in Figure $2 \mathrm{~b}$ (channel 2). The polaron is annihilated after the collision with the bipolaron, a channel that gives rise to a final state composed of a holebipolaron and a completely free electron. The effects caused by the temperature increase can be noted by first observing the diffusive pattern presented by the charge concentration of the quasi-particles, if compared with the case shown in Figure 2a. Also, the lattice oscillations imposed by the random forces are of such amplitudes that the polaron and the bipolaron can, as a matter of fact, be said to be of reduced stability, although it is still possible to identify such carriers immediately before the collision. After the collision, one can see that the once localized electron-polaron begins to spread over the lattice until it is completely delocalized at around 600 fs. This delocalization is the typical signature of the absence of the polaron structure in the system and the generation of a free electron. Another observed fact, due to temperature increasing, is that the collision process occurs $50 \mathrm{fs}$ earlier than the channel reported in Figure 2a. These results suggest a different value for the
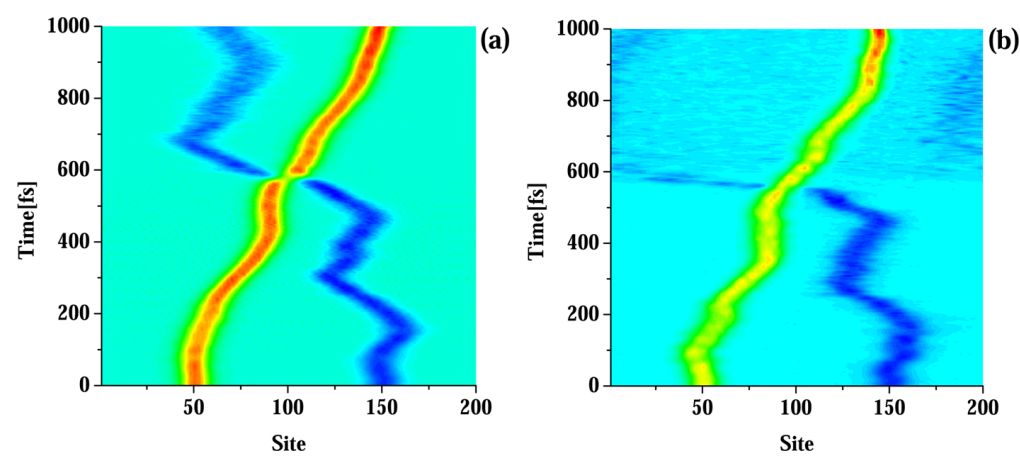

Figure 2. Mean charge density time evolution: (a) channel 1, electric field strength of $1.0 \mathrm{mV} / \AA$ and temperature of $75 \mathrm{~K}$; (b) channel 2, electric field strength of $1.5 \mathrm{mV} / \AA$ and $150 \mathrm{~K}$. 
critical electric field strength than that reported recently by Sun and collaborators. ${ }^{17}$ They have indicated that the critical electric field for the polaron-bipolaron scattering without a reaction that leads to a new state is of $0.8 \mathrm{mV} / \AA$. Our results present the formation of a polaron-exciton structure as a new state, for a critical field strength of $1.0 \mathrm{mV} / \AA$. Indeed, the markedly different behavior is caused by the temperature influence over the system, a kind of effect absent in the work of Sun. ${ }^{17}$ As discussed, the lattice vibrations dampened the charge carrier motion for electric field strengths to smaller than 1.0 $\mathrm{mV} / \AA$. This results in a transition for the quasi-particle from a drift driven dynamics by the electric field to a random walk dynamics imposed by the random thermal forces. In this way, we believe that an approach that takes into account the thermal effects over the charge carrier collision may provide a more realistic physical picture.

An important feature to recognize the new states formed after the polaron-bipolaron collision is the time evolution of the electronic occupation number of the intragap energy levels presented in Figure 3. Note that this figure shows the time

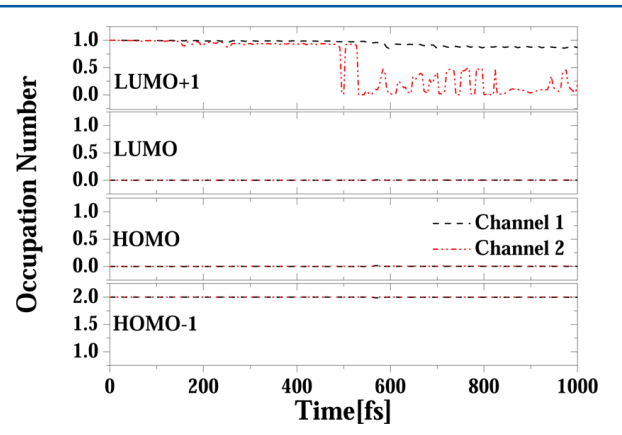

Figure 3. Time evolution of the occupation number for the intragap energy levels, where the black lines represents channel $1(75 \mathrm{~K})$ and the red lines channel $2(150 \mathrm{~K})$.

evolution of the occupation numbers for the intragap levels related to Figure 1. Considering the channel 1 (black lines in Figure 3), before the transient period in which the occupation number oscillates because of the collision, it is possible that the final state in LUMO + 1 level is not occupied by an integer number, which is evidence of partial electron excitation. Indeed, that fraction of the electron is excited to a higher level inside the conduction band, forming a bond state between the electron-polaron and the excitation characterizing the generation of a polaron-exciton structure, while the occupation of the other three levels remains the same. For channel 2 (red lines in Figure 3), after 500 fs the occupation number drops dramatically to zero, which indicates the complete annihilation of the polaron, leading to a free electron that is excited to the conduction band and the charge delocalization through the lattice. One can see that for the polaron annihilation process in channel 2, the occupation number for the other levels also remains the same as before the collision, showing mainly that the bipolaron keeps its integrity. In this way, these results indicate in general that an electron transfer from the polaron level LUMO + 1 to levels inside the conduction band will allow excited polarons only for very low temperature regimes (below $75 \mathrm{~K}$ ) and free carriers for temperatures higher than this critical value.
Whereas the occupation number analysis is the most suitable tool in studying the process that leads to the formation of excited polarons and free charges, the stability of the quasiparticles is better described by means of the energy levels time evolution. We finish our discussion by presenting in Figure 4

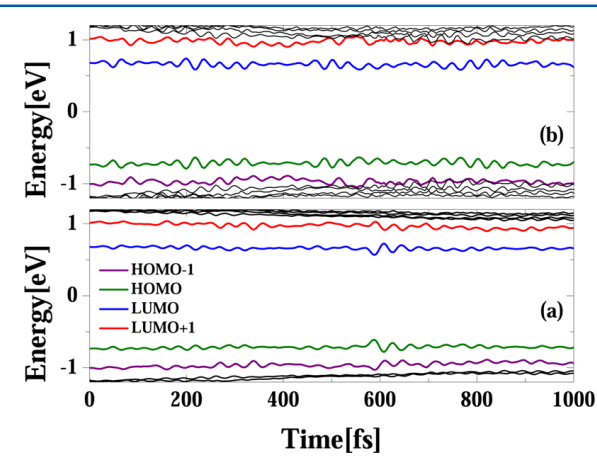

Figure 4. Time evolution of the energy levels for (a) channel 1, $75 \mathrm{~K}$ and (b) channel $2,150 \mathrm{~K}$.

the time evolution profile for the intragap energy levels of the simulations shown in Figure 2. As can be seem from Figure 4, at the beginning there are four intragap levels, which are localized electron states caused by the presence of a polaron and a bipolaron in the lattice. According to their wave functions, we know that the bipolarons energy levels present a larger narrowing when compared to those of the polaron. For clarity, we have used different colors where the polaron levels are represented by the purple (HOMO - 1) and red (LUMO +1 ) lines and those of the bipolarons by the green (HOMO) and blue (LUMO) lines. Figure $4 a$ depicts the process discussed in channel 1 , where a polaron-exciton structure is formed. The collisional process between the polaronbipolaron pair is noted to take place at about $600 \mathrm{fs}$, when the resulting phonons are represented by the little deeper oscillations of the energy levels inside the band gap. As both structures remain intact after the scattering process, one can see that there are four intragap levels until the end of the simulation. On the other hand, for processes discussed in channel 2, the signature of the loss of stability for the polaron is related to the red $($ LUMO +1$)$ and purple (HOMO -1$)$ states returning to the conducting and valence bands, respectively, as shown in Figure $4 \mathrm{~b}$. As in Figure $4 \mathrm{a}$, it is possible to note that the bipolaron remains stable through the rest of the simulation, since the green (HOMO) and blue (LUMO) bipolaron levels remain consistently inside the band gap. Furthermore, the oscillations presented by the energy levels result from the motion of the sites of the lattice influenced by the thermal random forces, as can be seen in Figure $4 \mathrm{a}$ and Figure $4 \mathrm{~b}$.

\section{SUMMARY AND CONCLUSIONS}

On the basis of a tight-binding electron-phonon interaction model modified to include an external electric field, electronelectron interactions, and temperature effects, the scattering processes between an electron-polaron and a hole-bipolaron have been simulated in a conjugated polymer lattice, using a nonadiabatic molecular dynamic method. The results show that there are two channels resulting from the scattering process between the polaron-bipolaron pair: channel 1 , below the 
critical temperature regime, where the positive bipolaron and a mixed stated composed of the negative polaron and an exciton are formed, and channel 2, where a positive bipolaron and a free electron are the resulting products of the collisional process, when the temperature is higher than the critical value. Furthermore, it is found that both channels depend sensitively on the strength of the applied electric field. These significant results reveal remarkable details concerning the polaronbipolaron recombination reaction and may enlighten the understanding of electroluminescence processes in polymer light emitting diodes.

\section{AUTHOR INFORMATION \\ Corresponding Authors \\ *L.A.R.J.: e-mail, luiju@ifm.liu.se. \\ *W.F.d.C.: e-mail, wiliam@unb.br. \\ Notes}

The authors declare no competing financial interest.

\section{ACKNOWLEDGMENTS}

The authors gratefully acknowledge the Swedish Research Council (VR) for financial support, the Swedish National Infrastructure for Computing (SNIC), and the financial support from the Brazilian Research Councils CNPq, CAPES, and FINATEC.

\section{- REFERENCES}

(1) Jailaubekov, A. E.; Willard, A. P.; Tritsch, J. R.; Chan, W.-L.; Sai, N.; Gearba, R.; Kaake, L. G.; Williams, K. J.; Leung, K.; Rossk, P. J.; Zhu, X.-Y. Hot Charge-Transfer Excitons Set the Time Limit for Charge Separation at Donor/Acceptor Interfaces in Organic Photovoltaics. Nat. Mater. 2013, 12, 66-73.

(2) White, M. S.; et al. Ultrathin, Highly Flexible and Stretchable PLEDs. Nat. Photonics 2013, 7, 811-816.

(3) Lanea, P. A.; Wei, X.; Vardeny, Z. V. Studies of Charged Excitations in pi-Conjugated Oligomers and Polymers by Optical Modulation. Phys. Rev. Lett. 1996, 77, 1544-1547.

(4) Bobbert, P. A.; Nguyen, T. D.; van Oost, F. W. A.; Koopmans, B.; Wohlgenannt, M. Bipolaron Mechanism for Organic Magnetoresistance. Phys. Rev. Lett. 2007, 99, 216801-4.

(5) Lei, J.; Sun, Z.; Zhang, Y.; Xie, S. Effect of Spin Polarization on Exciton Formation in Conjugated Polymers. Org. Electron. 2011, 10, $1489-1494$

(6) An, Z.; Di, B.; Wu, C. Q. Inelastic Scattering of Oppositely Charged Polarons in Conjugated Polymers. Eur. Phys. J. B 2008, 63, $71-77$.

(7) Sun, Z.; Stafström, S. Bipolaron Recombination in Conjugated Polymers. J. Chem. Phys. 2011, 135, 074902-7.

(8) Sun, Z.; Stafstöm, S. Spin-Dependent Polaron Recombination in Conjugated Polymers. J. Chem. Phys. 2012, 136, 244901-5.

(9) Di, B.; Meng, Y.; Wang, Y. D.; Liu, X. J.; An, Z. Formation and Evolution Dynamics of Bipolarons in Conjugated Polymers. J. Chem. Phys. B 2011, 115, 964-971.

(10) Di, B.; Meng, Y.; Wang, Y. D.; Liu, X. J.; An, Z. Electroluminescence Enhancement in Polymer Light-Emitting Diodes through Inelastic Scattering of Oppositely Charged Bipolarons. J. Chem. Phys. B 2011, 115, 9339-9344.

(11) Ribeiro, L. A.; da Cunha, W. F.; Neto, P. H. O.; Gargano, R.; e Silva, G. M. Impurity Effects on Polaron-Exciton Formation in Conjugated Polymers. J. Chem. Phys. 2013, 139, 174903-6.

(12) Li, Y.; Gao, K.; Sun, Z.; Yin, S.; sheng Liu, D.; jie Xie, S. Intrachain Polaron Motion and Geminate Combination in DonorAcceptor Copolymers: Effects of Level Offset and Interfacial Coupling. Phys. Rev. B 2008, 78, 014304-13.

(13) da Cunha, W. F.; Junior, L. A. R.; Gargano, R.; e Silva, G. M. Critical Temperature and Products of Intrachain Polaron Recombi- nation in Conjugated Polymers. Phys. Chem. Chem. Phys. 2014, 16, $17072-17080$

(14) Wei, X.; Hess, B. C.; Vardeny, Z. V.; Wudl, F. Studies of Photoexcited States in Polyacetylene and Poly(para-phenylenevinylene) by Absorption Detected Magnetic Resonance: The Case of Neutral Photoexcitations. Phys. Rev. Lett. 1992, 68, 666-669.

(15) Swanson, L. S.; Shinar, J.; Brown, A. R.; Bredley, D. D. C.; Friend, R. H.; Burn, P. L.; Kraft, A.; Holmes, A. B. Electroluminescence-Detected Magnetic-Resonance Study of Polyparaphenylenevinylene (PPV)-Based Light-Emitting Diodes. Phys. Rev. B 1992, 46, 15072-15077.

(16) Kadashchuk, A.; Arkhipov, V. I.; Kim, C. H.; Shinar, J.; Lee, D. W.; Hong, Y. R.; Jin, J.-I.; Heremans, P.; Bässler, H. Localized Trions in Conjugated Polymers. Phys. Rev. B 2007, 76, 235205-10.

(17) Sun, Z.; Li, Y.; Gao, K.; Liu, D.; An, Z.; Xie, S. Dynamical Study of Polaron-Bipolaron Scattering in Conjugated Polymers. Org. Electron. 2010, 11, 279-284.

(18) Onodera, Y. Polarons, Bipolarons, and Their Interactions in cisPolyacetylene. Phys. Rev. B 1984, 30, 775-785.

(19) Lima, M. P.; e Silva, G. M. Polaron Stability Under Collision with Different Defects in Conjugated Polymers. Int. J. Quantum Chem. 2006, 106, 2603-2608.

(20) Ribeiro, L. A.; da Cunha, W. F.; de Oliveira Neto, P. H.; Gargano, R; e Silva, G. M. Dynamical Study of Impurity Effects on Bipolaron-Bipolaron and Bipolaron-Polaron Scattering in Conjugated Polymers. J. Phys. Chem. B 2013, 117, 11801-11811.

(21) Su, W. P.; Schrieffer, J. R.; Heeger, A. J. Solitons in Polyacetylene. Phys. Rev. Lett. 1979, 42, 1698-1701.

(22) Su, W. P.; Schrieffer, J. R.; Heeger, A. J. Solitons Excitations in Polyeacetylene. Phys. Rev. B 1980, 22, 2099-2111.

(23) Brazovskii, S.; Kirova, N. Physical Theory of Excitons in Conducting Polymers. Chem. Soc. Rev. 2010, 39, 2453-2465.

(24) Stafström, S. Electron Localization and the Transition from Adiabatic to Nonadiabatic Charge Transport in Organic Conductors. Chem. Soc. Rev. 2010, 39, 2484-2499.

(25) Ribeiro, L. A.; da Cunha, W. F.; Neto, P. H. O.; Gargano, R.; e Silva, G. M. Effects of Temperature and Electric Field Induced Phase Transitions on the Dynamics of Polarons and Bipolarons. New J. Chem. 2013, 37, 2829-2836.

(26) Yu, Z. G.; Wu, M. W.; Rao, X. S.; Bishop, A. R. Excitons in Two Coupled Conjugated Polymer Chains. J. Phys.: Condens. Matter 1996, $8,8847-8857$.

(27) Neto, P. H. O.; da Cunha, W. F.; Teixeira, J. F.; Gargano, R.; e Silva, G. M. Electron-Lattice Coupling in Armchair Graphene Nanoribbons. J. Phys. Chem. Lett. 2012, 3, 3039-3042.

(28) Ribeiro, L. A.; da Cunha, W. F.; Neto, P. H. O.; Gargano, R.; e Silva, G. M. Predicting the Equilibrium Structure of Organic Semiconductors with Genetic Algorithms. Chem. Phys. Lett. 2013, $555,168-172$

(29) Ribeiro, L. A.; da Cunha, W. F.; Neto, P. H. O.; e Silva, G. M. Dynamics of Photogenerated Polaron-Excitons in Organic Semiconductors. Phys. Proc. 2012, 28, 112-116.

(30) Johansson, A.; Stafström, S. Polaron Dynamics in a System of Coupled Conjugated Polymer Chains. Phys. Rev. Lett. 2001, 86, 36023605 .

(31) Johansson, A.; Stafström, S. Nonadiabatic Simulations of Polaron Dynamics. Phys. Rev. B 2004, 69, 235205-7.

(32) An, Z.; Wu, C. Q.; Sun, X. Dynamics of Photogenerated Polarons in Conjugated Polymers. Phys. Rev. Lett. 2004, 93, 216407-4. (33) e Silva, G. M.; Terai, A. Dynamics of Solitons in Polyacetylene with Interchain Coupling. Phys. Rev. B 1993, 47, 12568-12577.

(34) e Silva, G. M. Electric-Field Effects on the Competition between Polarons and Bipolarons in Conjugated Polymers. Phys. Rev. B 2000, 61, 10777-10781.

(35) Ribeiro Junior, L. A.; da Cunha, W. F.; e Silva, G. M. SingletSinglet Exciton Recombination: Theoretical Insight into the Influence of High Density Regime of Excitons in Conjugated Polymers. J. Phys. Chem. B 2014, 118, 5250-5257. 
(36) Ribeiro, L. A.; Neto, P. H. O.; da Cunha, W. F.; Roncaratti, L. F.; Gargano, R.; da Silva Filho, D. A.; e Silva, G. M. Exciton Dissociation and Charge Carrier Recombination Processes in Organic Semiconductors. J. Chem. Phys. 2011, 135, 224901-224906.

(37) Ribeiro, L. A.; da Cunha, W. F.; Neto, P. H. O.; Gargano, R.; e Silva, G. M. Impurity Effects and Temperature Influence on the Exciton Dissociation Dynamics in Conjugated Polymers. Chem. Phys. Lett. 2013, 580, 108-114.

(38) Sun, Z.; Stafstöm, S. Dynamics of Exciton Dissociation in Donor-Acceptor Polymer Heterojunctions. J. Chem. Phys. 2013, 138, 164905-8.

(39) Neto, P. H. O.; da Cunha, W. F.; Roncaratti, L. F.; Gargano, R.; e Silva, G. M. Thermal Effects on Photogeneration of Free Carriers in Organic Conductors. Chem. Phys. Lett. 2010, 493, 283-287.

(40) Roncaratti, L. F.; Gargano, R.; de Oliveira Neto, P. H.; da Cunha, W. F.; da Silva Filho, D. A.; e Silva, G. M. TemperatureInduced Oscillating Electric Dipole in Conjugated Systems. Chem. Phys. Lett. 2012, 593, 214-217.

(41) Roncaratti, L. F.; Gargano, R.; e Silva, G. M. Theoretical Temperature Dependence of the Charge-Carrier Mobility in Semiconducting Polymers. J. Phys. Chem. A 2009, 113, 14591-14594. 


\title{
APPEND \\ PAPER IX
}

\section{Intrachain Polaron Recombination in Conjugated Polymers \\ Critical Temperature and Products of}

\author{
Wiliam Ferreira da Cunha \\ Luiz Antonio Ribeiro Junior \\ Ricardo Gargano \\ Geraldo Magela e Silva
}

Phys. Chem. Chem. Phys., 16, 17072-17080 (2014) 


\title{
PCCP
}

\section{PAPER}

\section{Critical temperature and products of intrachain polaron recombination in conjugated polymers}

Cite this: Phys. Chem. Chem. Phys. 2014, 16, 17072

Received 19th May 2014 Accepted 27th June 201

DOI: $10.1039 / \mathrm{c} 4 \mathrm{cp} 02184 \mathrm{c}$

www.rsc.org/pccp

Wiliam Ferreira da Cunha, ${ }^{a}$ Luiz Antonio Ribeiro Junior, ${ }^{* b}$ Ricardo Gargano ${ }^{a}$ and Geraldo Magela e Silva*a

\begin{abstract}
The intrachain recombination dynamics between oppositely charged polarons is theoretically investigated through the use of a version of the Su-Schrieffer-Heeger (SSH) model modified to include an external electric field, an extended Hubbard model, Coulomb interactions, and temperature effects in the framework of a nonadiabatic evolution method. Our results indicate notable characteristics concerning the polaron recombination: (1) it is found that there exists a critical temperature regime, below which an exciton is formed directly and (2) a pristine lattice is the resulting product of the recombination process, if the temperature is higher than the critical value. Additionally, it is found that the critical electric field regime plays the role of drastically modifying the system dynamics. These facts suggest that thermal effects in the intrachain recombination of polarons are crucial for the understanding of electroluminescence in optoelectronic devices, such as Polymer Light Emitting Diodes.
\end{abstract}

\section{Introduction}

Since the discovery of poly(paraphenylene vinylene) (PPV) and its electroluminescence properties, considerable amounts of effort have been devoted to study the photophysical applications of conjugated polymers for development of new technologies in organic optoelectronic devices. The potential advantages in terms of ease of synthesis, flexibility, low cost, and large-area capability make these materials attractive for the electronics industry, particularly when it comes to the promising development of a new display technology. This kind of system has been successfully implemented as the active component in various applications such as Organic Photovoltaics devices (OPVs) $)^{1}$ and Polymer Light Emitting Diodes (PLEDs). ${ }^{2}$ In these devices, the generation of excited states is the fundamental physical process involved. Particularly, polaron recombination is the key step behind the mechanism of electroluminescence in PLEDs. It is well known that when two polarons with opposite charges overlap in space, they will collide and recombine to form new species. The photon emission phenomenon depends sensitively on the radiative decay of excited species, which are formed by the reaction between the charge carriers. A considerable amount of theoretical work focused on understanding the processes underlying excited state formation in conjugated polymers through the collision mechanism of oppositely charged carriers has been

${ }^{a}$ Institute of Physics, University of Brasilia, 70.919-970, Brasilia, Brazil. E-mail: magela@fis.unb.br

${ }^{b}$ Department of Physics, Chemistry and Biology (IFM), Linköping University, SE-58183 Linköping, Sweden. E-mail: luiju@ifm.liu.se performed in the last few years. ${ }^{3-11}$ Nevertheless, studies that take into account the influence of temperature on such mechanisms remain theoretically unavailable and such processes are also very difficult to control experimentally.

Recently, An and coworkers used the Su-Schrieffer-Heeger (SSH) model to simulate the polaron recombination process in conjugated polymers. ${ }^{4}$ The goal was to identify the generation mechanism of the self-trapped polaron-exciton when only electric field effects are taken into account. Their results show that there are three regimes of the applied electric field to form new species. Li et $a .^{11}$ simulated the same process by adding Hubbard type electron-electron interactions to the SSH Hamiltonian. The simulations have pointed out that two polarons can recombine directly and efficiently in a full exciton, a process that is strongly dependent on the field strength. Sun and Stafström ${ }^{5}$ have used the SSH model to investigate the formation of excited states through the recombination process between two oppositely charged polarons in a system composed of two coupled conjugated polymer chains. In this case it is reported that, depending on the interchain distance and the electric field strength, the two polarons can either recombine into an exciton, become bound together forming a polaron pair or pass each other. Furthermore, it was found that the singlet states are always more easily formed than the triplet ones. These facts indicate that in PLEDs, the electroluminescence quantum efficiency can exceed the statistical limitation value of $25 \%$. Very recently, our previous research has shown that the presence of impurities in a conjugated polymer lattice, for several electric field regimes, favors the excited state formation thus improving the excitation yields when the scattering between 
Paper

oppositely charged polarons is considered. ${ }^{9}$ There are, however, still many controversial aspects regarding the influence of some physical processes such as temperature, electric field strength, coulomb interactions, and impurity effects on the formation of excited states via the recombination process between oppositely charged carriers, which requires detailed phenomenological descriptions. Of particular importance is the determination of critical values of temperature and the electric field in terms of the resulting products of collisions between quasi-particles.

In this paper, a systematic numerical investigation considering the influence of thermal effects, an external electric field and Coulomb interactions on the formation of excited states via recombination processes between an oppositely charged polaron pair is performed using a cis-polyacetylene chain in the framework of a nonadiabatic evolution method. An Ehrenfest Molecular Dynamics is performed by using a one-dimensional tightbinding model including lattice relaxation. Combined with the Extended Hubbard Model (EHM), a modified version of the SSH model is used to include the Brazovskii-Kirova symmetry breaking term. Temperature effects are included by means of a canonical Langevin equation. The aim of this work is to give a physical picture of the products derived from the recombination of oppositely charged polarons in conjugated polymers, when temperature effects are considered, and contribute to the understanding of these important processes, which may provide guidance to improve electroluminescence yields in PLDEs.

\section{Model and method}

A cis-polyacetylene chain is used to study the recombination process of two oppositely charged polarons in conjugated polymers. The overall Hamiltonian is given by $H=H_{\mathrm{SSH}}+H_{\mathrm{ee}}$. The first term is the SSH-type Hamiltonian ${ }^{12,13}$ modified to include an external electric field and the Brazovskii-Kirova symmetry-breaking, ${ }^{14}$ which has the following form:

$$
H_{\mathrm{SSH}}=-\sum_{n, s}\left(t_{n, n+1} C_{n+1, s}^{\dagger} C_{n, s}+\text { h.c. }\right)+\sum_{n} \frac{K}{2} y_{n}{ }^{2}+\sum_{n} \frac{p_{n}{ }^{2}}{2 M},
$$

where $n$ indexes the sites of the chain. The operator $C_{n, s}^{\dagger}\left(C_{n, s}\right)$ creates (annihilates) a $\pi$-electron state at the $n$th site with spin $s ; K$ is the harmonic constant that describes a $\sigma$ bond and $M$ is the mass of a $\mathrm{CH}$ group. The parameter $y_{n}$ is defined as $y_{n} \equiv u_{n+1}-u_{n}$, where $u_{n}$ is the lattice displacement of an atom at the $n$th site. $p_{n}$ is the conjugated momentum to $u_{n}$ and $t_{n, n+1}$ is the hopping integral, ${ }^{15}$ given by $t_{n, n+1}=\mathrm{e}^{-i \gamma A(t)}\left[\left(1+(-1)^{n} \delta_{0}\right) t_{0}-\alpha y_{n}\right]$, where $t_{0}$ is the hopping integral of a $\pi$-electron between nearest neighbouring sites in the undimerized chain, $\alpha$ is the electronphonon coupling, and $\delta_{0}$ is the Brazovskii-Kirova symmetrybreaking term, which is used to take the cis-symmetry of the polymer into account. $\gamma \equiv e a /(\hbar c)$, with $e$ being the absolute value of the electronic charge, $a$ is the lattice constant, and $c$ is the speed of light. The relation between the time-dependent vector potential $\mathbf{A}$ and the uniform electric field $\mathbf{E}$ is given by $\mathbf{E}=-(1 / c) \dot{\mathbf{A}} .^{16}$
View Article Online

PCCP

The last contribution denotes e-e interactions and can be written as

$$
\begin{aligned}
H_{\mathrm{ee}}= & U \sum_{i}\left(C_{i, \uparrow}^{\dagger} C_{i, \uparrow}-\frac{1}{2}\right)\left(C_{i, \downarrow}^{\dagger} C_{i, \downarrow}-\frac{1}{2}\right) \\
& +V \sum_{i}\left(n_{i}-1\right)\left(n_{i+1}-1\right),
\end{aligned}
$$

where $U$ and $V$ are the on-site and nearest-neighboring Coulomb repulsion strengths, respectively, and $n_{i}=C_{i, \uparrow}^{\dagger} C_{i, \uparrow}+C_{i, \downarrow}^{\dagger} C_{i, \downarrow}{ }^{17}$ It should be noted that the inclusion of the additional constant factors (related to the conventional description of the Hubbard model) is necessary in order to maintain the electron hole symmetry of the Hamiltonian. The many-body problem is treated using the unrestricted Hartree-Fock (UHF) approximation. The single determinant considered in this formalism is built through the use of a number of single-particle orbitals, which are optimized according to the variational principle. A modified version of the SSH model that includes Coulomb interactions using an UHF approach can capture the essential electronic properties of quasi-particle transport in conjugated polymers, and has been successfully applied in many relevant studies of the field for over three decades. ${ }^{5,18,19}$ The parameters used here are $t_{0}=2.5 \mathrm{eV}, M=1349.14 \mathrm{eV} \times \mathrm{fs}^{2} \AA^{-2}, K=21 \mathrm{eV} \AA^{-2}, \delta_{0}=0.05$, $\alpha=4.1 \mathrm{eV} \AA^{-1}, a=1.22 \AA$, and a bare optical phonon energy $\hbar \omega_{Q}=\hbar \sqrt{4 K / M}=0.16 \mathrm{eV}$. These values have been used in previous simulations and are expected to be valid for conjugated polymers in general. ${ }^{20-26}$

In order to solve these equations numerically, first a stationary state that is self-consistent with all degrees of freedom of the system (lattice and electrons) is obtained. The initial bond configuration and the electronic structure of a polymer chain containing a hole-polaron and an electron-polaron can be obtained by solving the following self-consistent equations of the bond configuration $\left\{u_{n}\right\}$ and the electronic wave functions $\left\{\phi_{n}\right\}$ :

$$
u_{n-1}-u_{n}=-\frac{2 \alpha}{K} \sum_{\mu} \phi_{\mu}(n) \phi_{\mu}(n+1)+\frac{2 \alpha}{N K} \sum_{\mu, n} \phi_{\mu}(n) \phi_{\mu}(n+1),
$$

$\varepsilon_{\mu} \phi_{\mu}(n)=-\left[t_{0}-\alpha\left(u_{n-1}-u_{n}\right)\right] \phi_{\mu}(n+1)-\left[t_{0}-\alpha\left(u_{n}-u_{n-1}\right)\right] \phi_{\mu}(n-1)$

where $\varepsilon_{\mu}$ is the eigenvalue of $\mu$ th energy level. We begin by constructing the Hamiltonian from a $\left\{y_{n}\right\}$ set of positions, that is, a composition of two isolated polarons. By solving the timeindependent Scrhödinger equation, a new set of coordinates $\left\{y_{n}^{\prime}\right\}$ is obtained. Iterative repetitions of this procedure yield a self-consistent initial state when $\left\{y_{n}\right\}$ is close enough to the solution. The equation of motion that describes the site displacement and provides the temporal evolution of the lattice is obtained by a classical approach. The nuclear dynamics is carried out with the Euler-Lagrange equations

$$
\frac{\mathrm{d}}{\mathrm{d} t}\left(\frac{\partial\langle L\rangle}{\partial \dot{u}_{n}}\right)-\frac{\partial\langle L\rangle}{\partial u_{n}}=0
$$


PCCP

View Article Online

Paper

where $\langle L\rangle=\langle T\rangle-\langle V\rangle$. Eqn (5) leads to a newtonian equation $M \ddot{u}_{n}=F_{n}(t)$. Thus,

$$
\begin{aligned}
F_{n}(t)=M \ddot{u}_{n}(t)= & -K\left[2 u_{n}(t)-u_{n+1}(t)-u_{n-1}(t)\right]+\alpha\left[B_{n, n+1}(t)\right. \\
& \left.-B_{n-1, n}(t)+B_{n+1, n}(t)-B_{n, n-1}(t)\right],
\end{aligned}
$$

where $F_{n}(t)$ represents the force on the $n$th site. ${ }^{27}$ Here, $B_{n, n^{\prime}}(t)=\sum_{k, s} \psi_{k, s}{ }^{*}(n, t) \psi_{k, s}\left(n^{\prime}, t\right)$ is the term that couples the electronic and lattice solutions. The primed summation represents a sum over occupied states.

The time dependent wave functions are constructed by means of a linear combination of instantaneous eigenstates of the electronic Hamiltonian. The solutions of the time-dependent Schrödinger equation can be put in the form

$$
\begin{aligned}
\psi_{k, s}\left(n, t_{j+1}\right)= & \sum_{l}\left[\sum_{m} \phi_{l, s}^{*}\left(m, t_{j}\right) \psi_{k, s}\left(m, t_{j}\right)\right] \\
& \times \mathrm{e}^{\left(-i \varepsilon_{l} \Delta t / \hbar\right)} \phi_{l, s}\left(n, t_{j}\right) .
\end{aligned}
$$

$\left\{\phi_{l}(n)\right\}$ and $\left\{\varepsilon_{l}\right\}$ are the eigenfunctions and the eigenvalues of the electronic part for the Hamiltonian (within the unrestricted Hartree-Fock approximation) at a given time $t_{j}{ }^{28}$ Eqn (6), which governs the evolution of the lattice system, can be numerically integrated using the method

$$
\begin{gathered}
u_{n}\left(t_{j+1}\right)=u_{n}\left(t_{j}\right)+\dot{u}_{n}\left(t_{j}\right) \Delta t, \\
\dot{u}_{n}\left(t_{j+1}\right)=\dot{u}_{n}\left(t_{j}\right)+\frac{F_{n}\left(t_{j}\right)}{M} \Delta t .
\end{gathered}
$$

Hence, the electronic wave functions and the lattice displacements at the $(j+1)$ th time step are obtained from the $j$ th time step. At time $t_{j}$ the wave function $\left\{\psi_{k, s}\left(i, t_{j}\right)\right\}$ is expressed as an expansion of the eigenfunctions $\left\{\phi_{l, s}\right\}$ at that moment: $\psi_{k, s}\left(i, t_{j}\right)=\sum_{l=1}^{N} C_{l, k}^{s} \phi_{l, s}(i)$, where $C_{l, k}^{s}$ are the expansion coefficients. The occupation number for each eigenstate $\phi_{l, s}$ is $\eta_{l, s}\left(t_{j}\right)=\sum_{k}^{\prime}\left|C_{l, k}^{s}\left(t_{j}\right)\right|^{2} \cdot \eta_{l, s}\left(t_{j}\right)$ contains information concerning the redistribution of electrons among the energy levels. ${ }^{9}$

Here, the temperature effects are simulated by adding thermal gaussian random forces with a zero mean value $\left\langle\zeta_{n}(t)\right\rangle \equiv 0$ and variance $\left\langle\zeta_{n}(t) \zeta_{n}\left(t^{\prime}\right)\right\rangle=2 k_{\mathrm{B}} T \gamma M \delta\left(t-t^{\prime}\right)$. We adopted a white stochastic signal $\zeta_{n}(t)$ as the fluctuation term. Also, in order to keep the temperature constant at its initial value after a transient period (named thermalization), it is necessary to introduce a damping factor, $\gamma$. Therefore, eqn (6) is modified to $M \ddot{u}=-\gamma \dot{u}+\zeta_{n}(t)+F_{n}(t)$. The modified eqn (4) no longer defines a set of ordinary differential equations (ODEs); rather, in this formalism we deal with a set of stochastical differential equations (SDEs). It is then important to find a proper integrator for solving SDEs. Since our model assumes a classical treatment for the lattice, it is possible to use the regular Langevin-type approach to take thermal effects into account. Various discretizations of the modified eqn (6) are available in the literature. We have used the velocity-verlet (eqn (6) and (7)) that is very similar to the popular BBK integrator. ${ }^{29}$ Furthermore, we have introin such a way to possess the power spectral density given by the fluctuation-dissipation theorem. In this way, the fluctuations can be obtained by using

$$
\zeta_{n}(t)=\sqrt{\left(2 k_{\mathrm{B}} T \gamma M\right) / \Delta t} \times Z^{n},
$$

where $Z^{n}$ is a random number. The damping constant can be determined by low temperature lattice thermal conductivity measurements. The $\gamma$ value used here has the same order of magnitude as expected from experimental data of the Raman spectral line width in polydiacetylene $\left(\gamma=0.01 \omega_{Q}\right) \cdot{ }^{30}$ It should be emphasized that this procedure of including temperature effects by means of a Langevin formalism has been extensively used in the literature and is known to yield excellent qualitative results. $^{31-37}$

\section{Results}

A systematic numerical investigation concerning the influence of temperature effects, an external electric field, and Coulomb interactions over the recombination dynamics of two oppositely charged polarons is performed in systems composed of 200-site cis-polyacetylene chains with periodic boundary conditions. For the electric field, turned on quasi-adiabatically, ${ }^{32}$ the values used in the simulations varied from 0.1 to $2.0 \mathrm{mV} \AA^{-1}$ with an increment of $0.1 \mathrm{mV} \AA^{-1}$, whereas the temperature regimes considered ranged from 0 to $200 \mathrm{~K}$ with a step of $10 \mathrm{~K}$. For the on-site electron-electron interactions the value considered is $U=0.2 t_{0}$. The nearest-neighboring Coulomb repulsion strength is determined by using the relation $V=U / 2$. It is worthy to mention that, although some relevant studies have reported results using $U$ levels higher than $4.0 \mathrm{eV},{ }^{34,38}$ recently a considerable amount of studies has been performed considering moderated values of electron-electron interactions in a range of $0.5-3.0$ eV. $^{3,5,7-9,11,18,19,39-44}$ In this context, Fig. 1 presents the schematic diagram of energy levels for a lattice containing a hole-polaron (positive carrier) and an electron-polaron (negative carrier). These charge carriers are formed by removing or adding electrons to the polymer chain, causing lattice distortions and the increase of energy levels to the inside of the band gap. A single polymer chain containing a hole-polaron is represented by the absence of one electron in the HOMO level and the absence of two electrons in the LUMO +1 level, which yields a $+1 e$ charge. On the other hand, an electron-polaron is represented by the levels HOMO - 1 and LUMO, in which the upper one is occupied by one electron and the lower one is occupied by two electrons, yielding a $-1 e$ charge.

The discussion concerning the products of the collision process between oppositely charged carriers starts by the analysis of the polaron-polaron interaction under a temperature regime of $50 \mathrm{~K}$ and an electric field strength of $1.5 \mathrm{mV}^{-1}$, as shown in Fig. 2. The temporal evolution of the staggered bond-length, $\bar{y}(t)=(-1)^{n}\left[y_{n-1}(t)+2 y_{n}(t)+y_{n+1}(t)\right] / 4$, depicts the dynamical process. An excited state is formed directly after the collision duced both the dissipative force and the gaussian random force 

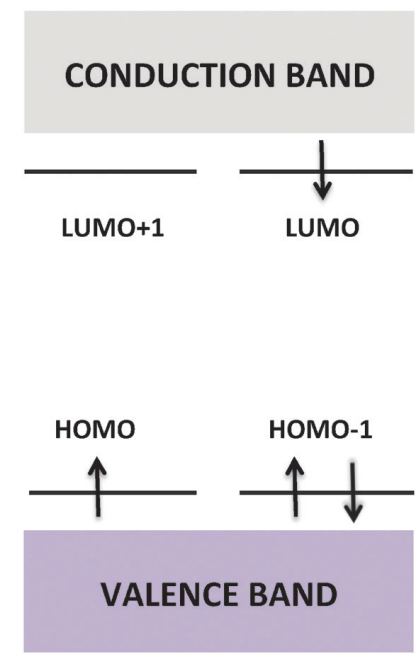

Fig. 1 The schematic diagram of energy levels for a polymer chain containing an electron-polaron and a hole-polaron.
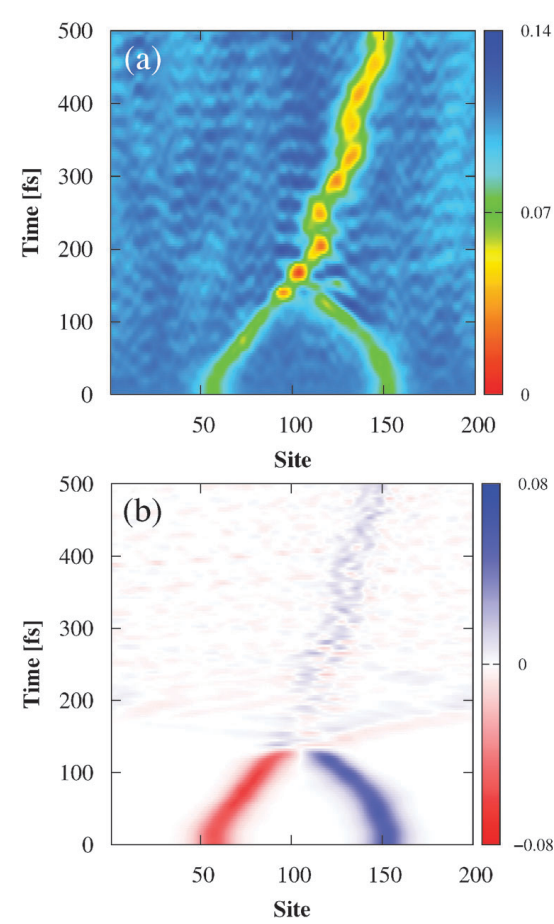

Fig. 2 (a) Staggered bond-length and (b) mean charge density time evolution for an electric field strength of $1.5 \mathrm{mV} \AA^{-1}$ and a temperature regime of $50 \mathrm{~K}$.

between the polaron pair, as presented in Fig. 2(a) (channel 1). Initially, the electron-polaron is located at the 60th site while the hole-polaron is at 150th site. The two charge carriers are separated far enough from each other so that they behave as independent structures. After a small transient time for the electric field response, the polarons begin to move towards one another due to the different charges. The polaron dynamics before the collisional processes occurs as already described in the literature..$^{33,37}$ At approximately $150 \mathrm{fs}$, when the collision takes place, one can see that the electron-polaron and the holepolaron recombine into a well-defined lattice structure from the lattice distortion point of view. This process, however, results in the formation of only one neutral excited state after the collision. Furthermore, it can be seen that after about $50 \mathrm{fs}$, the adding up of thermal energy begins to become visible through the blurring of the figure (cyan regions). The interaction between the two original polarons produces phonons after the collision that, combined with the thermal oscillations, generates even more energetic phonons represented mainly by the dark blue regions closer to the site where the collision has occurred, as shown in Fig. 2(a). Another important aspect that can be noted in this figure is obtained by comparing the lattice deformation of the excited state generated after the collision with the lattice defect that characterizes any of the polarons at the beginning of the simulation. The lattice distortion for the exciton is wider than that of the polarons, which shows that the distortion for neutral excitations involve a higher number of sites. Also, the red and yellow regions, not present in the polarons lattice defects, indicate a higher level of deformation of the lattice, which suggests that excitons are more stable structures than polarons. ${ }^{5}$

Fig. 2(b) depicts the temporal evolution of mean charge density $\bar{\rho}(t)=1-\left[\rho_{n-1}(t)+2 \rho_{n}(t)+\rho_{n+1}(t)\right] / 4$ for the polaron pair collision in this first channel - the recombination process reported so far. Immediately after their encounter, the charges of the two polarons cancel each other. It can be conjectured that the charge cancelation process occurs due to excited state formation. Also, it is possible to note small charge density fluctuations associated with the localized excitation, where the positive and negative charges are bounded together. Indeed, the charge density alternates across the lattice deformation for the localized excited state, creating an oscillating molecular electric dipole. Recently, Silva and coworkers reported a similar behavior where a neutral soliton showed an infrared signature that comes from a temperature-induced electric dipole on the lattice deformation of that well-localized excitation. ${ }^{36}$ This fact may suggest that the oscillating electric dipoles induced by temperature effects are some sort of general features when neutral excitations are considered. It is important to note that this channel of polaron pair recombining into a neutral structure is only observed for electric fields strengths between $0.8-1.5 \mathrm{mV}^{-1}$ and temperature regimes of $50-160 \mathrm{~K}$. It is due to the fact that the temperature damps the polaron motion avoiding the interaction between them. ${ }^{33}$ Furthermore, the simulation presented in Fig. 2(b) yields a neutral excited state with a better rate than those previously reported by An and colleagues, ${ }^{4}$ where exactly the same kind of calculations were performed in the absence of Coulomb interactions and temperature effects. In An's studies, for an electric field strength 


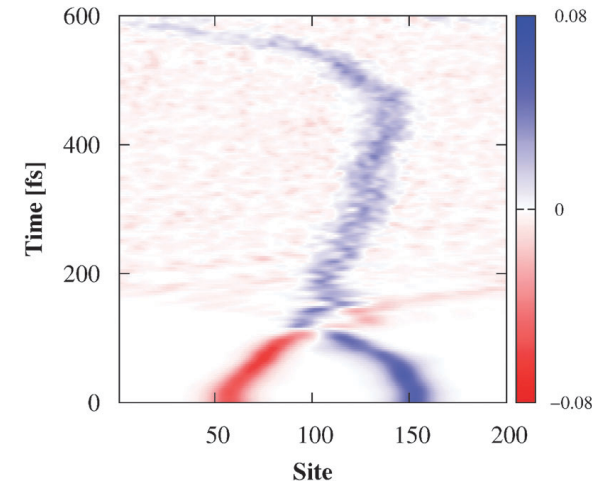

Fig. 3 Mean charge density time evolution for an electric field strength of $1.5 \mathrm{mV} \AA^{-1}$ and a temperature regime of $200 \mathrm{~K}$

of $1.2 \mathrm{mV} \AA^{-1}$, it was observed that an inelastic scattering process occurs between oppositely charged polarons to form a mixed state composed of polarons and excitons. The lattice fluctuations and the high energy of phonons provided by the inclusion of thermal effects, absent in An's work, are responsible for this better yield of neutral excitations.

The influence of temperature on the collisional process of the charge carriers can be better considered usng a thermal bath with temperature four times higher than the one considered in channel 1 and also an electric field strength of $1.5 \mathrm{mV}^{-1}$, as shown in Fig. 3 (channel 2). For the sake of conciseness we chose to present only the results of mean charge density evolution, for these are more instructive than the bond length evolution. In this case it can be seen that the electron-polaron is annihilated immediately after the collision, whereas the hole-polaron is not completely dissociated before $600 \mathrm{fs}$. This channel gives rise to a final state where a dimerized lattice is obtained. The effects of the temperature increase can be readily noted by comparing the diffusive pattern presented by the charge concentration of the charge carriers to that of Fig. 2(b). Also, the lattice oscillations imposed by the random forces are of such amplitudes that the polarons have reduced stability. The charge delocalization presented in Fig. 3 is the typical signature of the absence of the polaron structure in the system, which leads to a dimerized lattice. These results show that there are different products formed after the collisional process depending on temperatures and electric field strength. On one hand this is consistent with the critical electric field strength reported recently by An and collaborators for the formation of a polaron-exciton structure, ${ }^{4}$ but on the other hand the more realistic description we have made resulted in different values from the quantitative point of view. Our results point towards a critical field strength of $1.5 \mathrm{mV} \AA^{-1}$ where only neutral excitations are formed. Indeed, the markedly different behavior is caused by the influence of temperature on the system, a kind of effect absent in the work of An. ${ }^{4}$ The lattice vibrations damp the charge carrier motion for electric field strengths smaller than $1.5 \mathrm{mV} \AA^{-1}$, as already discussed, resulting in a transition of the quasi-particle dynamics from a drift driven by the electric field to a random walk dynamics imposed by the random forces.

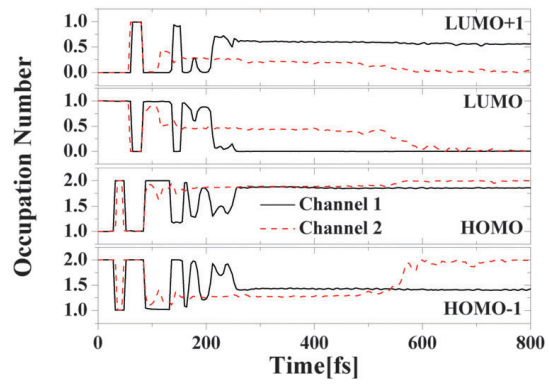

Fig. 4 Time evolution of the occupation number of the intra-gap energy levels, where the black lines represent channel $1\left(50 \mathrm{~K}\right.$ and $\left.1.5 \mathrm{mV} \AA^{-1}\right)$ and the red lines depict channel $2\left(200 \mathrm{~K}\right.$ and $2.0 \mathrm{mV}^{-1}$ ).

This can be responsible for the differences in the final products obtained here. In this way, we believe that an approach that takes into account thermal effects over the charge carrier collision is crucial for the correct description of more realistic polymers, which are suitable for actual technological applications.

The previous discussion on the creation of neutral excited states is confirmed by an analysis of the occupation number presented in Fig. 4. Note that this figure shows the time evolution of the occupation numbers for the intra-gap levels related to Fig. 1. Initially, there are four levels inside the band gap, which are localized electron states corresponding to the two lattice defects. At the beginning of the process (first few $100 \mathrm{fs}$ ) these energy levels are degenerated because the two polarons are initially far apart. During this transient regime, the occupation number of the LUMO level sharply decreases from 1 to 0 ; simultaneously, the occupation number of LUMO + 1 sharply increases from 0 to 1 . This change in the occupation number reflects the fact that these two levels interchange positions, a process that is energetically accessible due to the degeneracy. The electron transfer associated with this change is mostly due to the fact that the wavefunction corresponding to this level is spread over the whole chain. This process occurs in a similar fashion between HOMO -1 and HOMO levels. Once again, these changes do not correspond to the transfer of an electron from one level to another; instead, they occur as a result of changes in the eigenenergies of the respective levels. After $200 \mathrm{fs}$, the degeneracy is broken by the interplay between the electric field and the electron-electron interactions and by the coupling between the two polarons when they are close to each other. From that moment, the interchange between the positions of the energy levels is no longer observed. Very recently, a similar behavior for abrupt changes in the occupation number was reported by Sun and Stafström. ${ }^{34}$ Considering channel 1 (black lines in Fig. 4), before the transient period in which the occupation number oscillates due to the collision, it is possible to note that the final state in the LUMO + 1 level is not covered by an integer number, which is evidence of partial electron transfer from the LUMO level. Since the LUMO level stays completely empty after 300 fs the remaining fraction of this level, which was not transferred to LUMO +1 , decays to the HOMO level resulting in an photon emission process. Similar to the occupation process for the 
LUMO +1 , the HOMO level receives the electron fraction transferred from the HOMO - 1, while the occupation of the HOMO - 1 level remains unchanged after the partial transfer. For channel 2 (red lines in Fig. 4), after 600 fs the occupation number for the LUMO level drops to zero (a photon emission process) which indicates the complete annihilation of the polaron, whereas the HOMO level is fully occupied by two electrons. Both HOMO - 1 and HOMO levels are occupied by two electrons after $600 \mathrm{fs}$; on the other hand the LUMO and LUMO + 1 levels become empty. This final configuration for the occupation numbers reported in channel 2 results in a dimerized lattice where the polaron defect is absent. In this way, these results indicate that, in general, the combination of the mechanisms of partial electron transfer among the levels together with a photon emission will induce the formation of neutral states only for low temperature regimes (below $150 \mathrm{~K}$ ). Whenever the temperature is higher than this critical value a dimerized lattice is observed to take place.

While the occupation number analysis is the most suitable tool for studying the process that leads to the formation of new

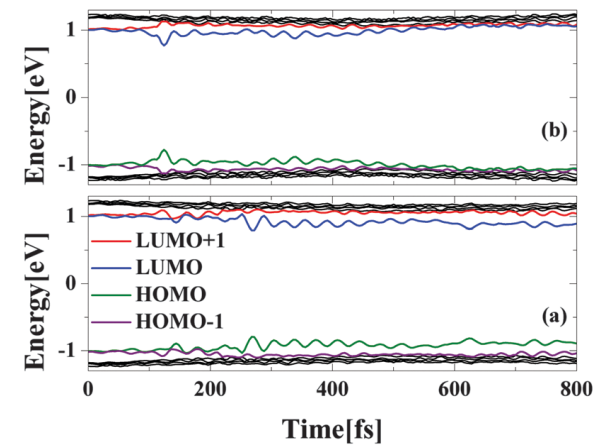

Fig. 5 Time evolution of the energy levels for (a) channel 1-50 K and (b) channel 2-200 K considering an electric field strength of $1.5 \mathrm{mV} \AA^{-1}$. species such as excited states, the stability of the quasi-particles is better described by means of the energy level time evolution. We finish our discussion by presenting in Fig. 5 the time evolution profile of the intra-gap energy levels of the simulations shown in Fig. 2 and 3. As can be seen from Fig. 5, at the beginning of the simulation there are four intra-gap levels, which are electron states caused by the presence of the polarons in the lattice. Fig. 5(a) depicts the process discussed in channel 1 , where an exciton is formed directly. At about $50 \mathrm{fs}$, the degenerate levels rapidly become nondegenerated due to the influence of temperature and the electron-electron interactions. The collisional process between the two polarons is noted to take place at about $200 \mathrm{fs}$, when the resulting phonons are associated with the slightly deeper oscillations of the energy levels inside the band gap. As both polarons are annihilated and one exciton arises after the interaction between the charge carriers, one can see that the two intra-gap levels HOMO - 1 and LUMO + 1 return to the valence and conduction bands, respectively. On the other hand, the HOMO and LUMO levels remain inside the band gap until the end of the simulation, presenting a new configuration that is located deeper inside the gap than at the beginning. According to their wave functions, we know that the exciton energy levels present a larger narrowing than those of polarons. This suggests that excitons are structures more stable than polarons. Regarding the process discussed in channel 2, the signature of the loss of stability of the polarons, that leads to a dimerized, lattice is related to the LUMO and HOMO levels returning to the conducting and valence bands, respectively, as shown in Fig. 5(b). Furthermore, the oscillations presented by the energy levels result from the motion of the sites of the lattice influenced by the thermal random forces, as can be seen in Fig. 5(a) and (b).

As discussed above, the polaron-polaron reaction induces electron redistribution among levels, i.e., there are other different electronic states after the collisional process between these charge carriers. As shown in Fig. 4 and 5, we found that there are mainly two new electronic states after collision, when temperature effects are taken into account, which are shown in Fig. 6.

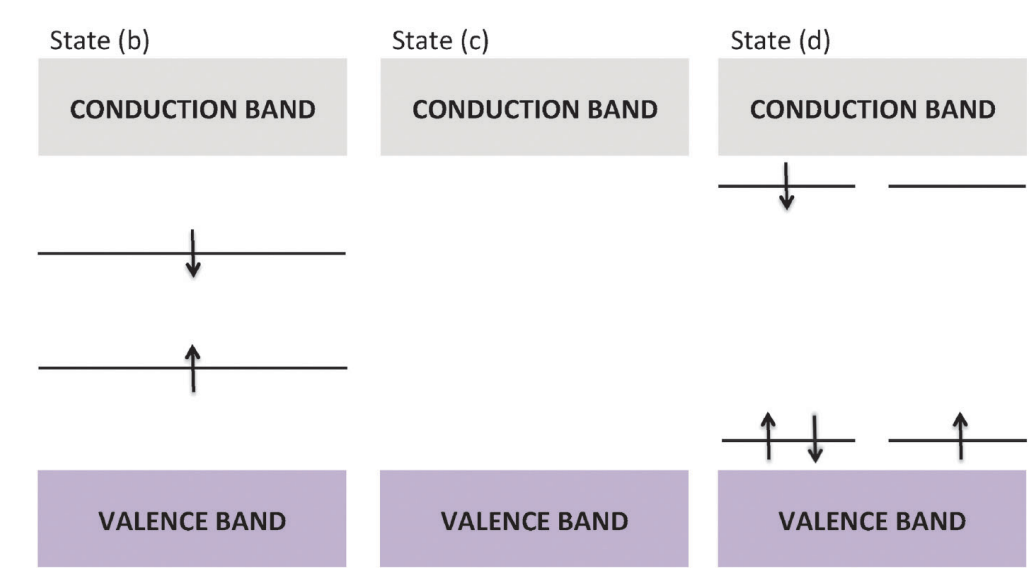

Fig. 6 The schematic diagram of energy levels for a polymer chain (b) containing a neutral excitation, (c) considering a pristine lattice, and (d) containing two oppositely charged polaron-excitons. 
However, a third electronic state (named polaron-exciton) can occur after a reactive scattering between an oppositely charged polaron-pair as mentioned above and reported in recent studies. ${ }^{3,4,6,9-11}$ Here, state (a) denotes the initial electron distribution, represented in Fig. 1, which contains an oppositely charged polaron-pair. State (b) denotes the final electronic configuration after the formation of a neutral excited state (channel 1). The dimerized lattice, channel 2, is represented in state (c). Finally, state (d) denotes the intra-gap configuration for a system that contains two polaron-excitons.

We now discuss the yields for these states using a projection method. ${ }^{18}$ After each evolution step, the lattice displacements $\left\{u_{n}\right\}$ are determined, and the Hartree-Fock Hamiltonian of the system is also obtained. One can, then, obtain all single electron eigenwavefunctions $\left\{\phi_{i}\right\}$ by diagonalizing the Hamiltonian matrix.

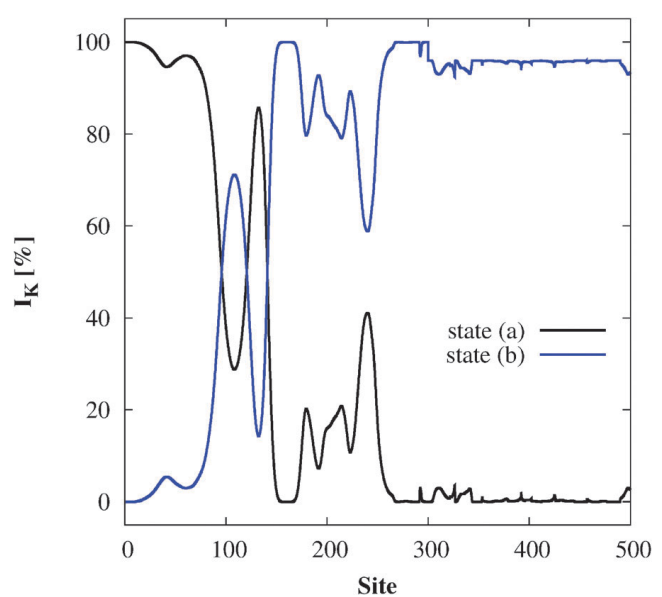

Fig. 7 Time dependence of the yields for states (a) and (b) which are shown in Fig. 1 and 6, respectively.
Any desired eigenstate of the system can thus be constructed using these single electron wavefunctions in the form of a Slater determinant, $\left|\Phi_{k}\right\rangle=\left|\phi_{1 \downarrow} \phi_{1 \uparrow} \phi_{2 \downarrow} \phi_{2 \uparrow} \ldots\right\rangle$. For example, the ground state involves all single electron states in the valence band. Here, the desired eigenstate is the neutral excited state. The yield of the eigenstates $\left|\Phi_{k}\right\rangle$ is obtained by projecting it on the evolutional wave function $|\Psi(t)\rangle$ which is also a Slater determinant constructed by single electron evolutional wave functions $\left\{\psi_{j}(t)\right\}$, $\left|\Psi_{k}\right\rangle=\left|\psi_{1 \downarrow} \psi_{1 \uparrow} \psi_{2 \downarrow} \psi_{2 \uparrow} \ldots\right\rangle$, i.e., $I_{K}=\left|\left\langle\Phi_{k} \mid \Psi(t)\right\rangle\right|^{2} \cdot{ }^{45,46}$ Fig. 7 shows the time dependence of the yields for states (a) and (b) during the polaron-polaron interaction. It can be seen that the yield of state (a) remains at $100 \%$ before 80 fs. This fact denotes that the polarons do not interact during this period. From 80 to $200 \mathrm{fs}$, the yield of state (a) drops about 30\% whereas state (b) increases approximately $70 \%$, with some fluctuations resulting from the collisional process. This suggests that, in this regime, these states are mixed. After $300 \mathrm{fs}$, the yield of state (a) sharply drops to zero. At the same time, the yield of state (b) increases to about $95 \%$. This explicitly shows that state (b) is mostly the unique state after the collision. As previously discussed, state (b) (channel 1) contains a neutral excited state. The remaining yield of state (a), which was not converted into state (b) $(\sim 5 \%)$, denotes a free carrier, i.e., the polaron-polaron interaction may transfer electrons from the intra-gap energy levels to the conduction band with some probability after collision. Thus, the results indicate that an excited neutral state and a free carrier can be produced by the polaron-polaron reaction.

We have calculated the yields of state (a) for different electric field and temperature regimes. The results are displayed in Fig. 8. The blue region denotes the regimes where the interplay between the electric field and temperature is not favorable for the formation of neutral excited states, i.e., the yield values are zero. Below the critical electric field strength of $0.8 \mathrm{mV}^{-1}$ and for temperature regimes smaller than $50 \mathrm{~K}$, the interplay between these two effects favors the polaron-exciton formation (state (d)) in the same way as reported in ref. 3 and 4 . If the temperature lies

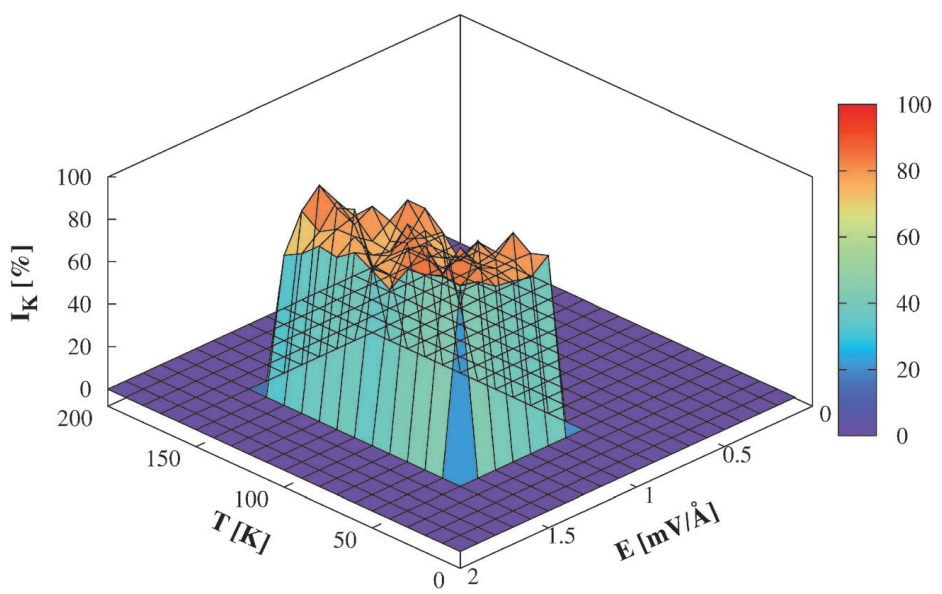

Fig. 8 Final yield values for state (a) after 1 ps as a function of the electric field strength and temperature effects. 
Paper

in the regime $50-200 \mathrm{~K}$, the polaron motion is damped and the reaction between them is no longer observed. However, just above the critical electric field strength, about $1.5 \mathrm{mV} \AA^{-1}$, the yield of state (a) sharply increases to about 60-95\%, considering a temperature regime that lies in the $50-160 \mathrm{~K}$ regime. It shows that the electric field strength dominates the motion of the polarons over the random motion imposed by the thermal effects. The abrupt change in the yield values of the species formed after the interaction between the oppositely charged carriers is recognized as a standard behavior of this process. ${ }^{3,18,47}$ Considering electric field strengths higher than $1.5 \mathrm{mV} \AA^{-1}$, the collision process annihilates the polarons producing a final state (state (c)) where a dimerized lattice is obtained. Also, the lattice oscillations imposed by the random forces are of such amplitudes that the polarons have reduced stability, as mentioned before. Considering temperatures higher than $200 \mathrm{~K}$, the interplay between the electric field and temperature annihilates the polaron, even before the collision process, as reported in the reference. ${ }^{33}$

\section{Conclusions}

In summary, using a tight-binding electron-phonon interaction model modified to include an external electric field, electronelectron interactions, and temperature effects the recombination processes between an electron-polaron and a hole-polaron have been simulated in a conjugated polymer lattice, by means of nonadiabatic molecular dynamics. The results show that there are two channels resulting from the recombination between the polarons: channel 1 , where a critical temperature regime exists, below which neutral excitation directly forms channel 2 , where a dimerized lattice is the resulting product of the collisional process for temperatures higher than the critical value. Furthermore, it is found that both channels depend sensitively on the strength of the applied electric field. These significant results reveal remarkable details concerning the polaron recombination reaction and provide guidance to understand electroluminescence processes in Polymer Light Emitting Diodes.

\section{Acknowledgements}

The authors gratefully acknowledge the Swedish Research Council (VR) for financial support, the Swedish National Infrastructure for Computing (SNIC), and the financial support from the Brazilian Research Councils CNPq, CAPES and FINATEC.

\section{References}

1 A. E. Jailaubekov, A. P. Willard, J. R. Tritsch, W.-L. Chan, N. Sai, R. Gearba, L. G. Kaake, K. J. Williams, K. Leung and P. J. Rossk, et al., Nat. Mater., 2013, 12, 66.

2 M. S. White, M. Kaltenbrunner, E. D. Gowacki, K. Gutnichenko, G. Kettlgruber, I. Graz, S. Aazou, C. Ulbricht, D. A. M. Egbe and M. C. Miron, et al., Nat. Photonics, 2013, 7, 811.

3 J. Lei, Z. Sun, Y. Zhang and S. Xie, Org. Electron., 2011, 10, 1489.
View Article Online

PCCP

4 Z. An, B. Di and C. Q. Wu, Eur. Phys. J. B, 2008, 63, 71.

5 Z. Sun and S. Stafström, J. Chem. Phys., 2011, 135, 074902.

6 Z. Sun and S. Stafstöm, J. Chem. Phys., 2012, 136, 244901.

7 B. Di, Y. Meng, Y. D. Wang, X. J. Liu and Z. An, J. Phys. Chem. B, 2011, 115, 964.

8 B. Di, Y. Meng, Y. D. Wang, X. J. Liu and Z. An, J. Phys. Chem. B, 2011, 115, 9339.

9 L. A. Ribeiro, W. F. da Cunha, P. H. O. Neto, R. Gargano and G. M. e Silva, J. Chem. Phys., 2013, 139, 174903.

10 Q. Lu, H. Zhao, Y. Chen and Y. Yan, Physica B, 2013, 412, 13.

11 Y. Li, K. Gao, Z. Sun, S. Yin, D. sheng Liu and S. jie Xie, Phys. Rev. B: Condens. Matter Mater. Phys., 2008, 78, 014304.

12 W. P. Su, J. R. Schrieffer and A. J. Heeger, Phys. Rev. Lett., 1979, 42, 1698.

13 W. P. Su, J. R. Schrieffer and A. J. Heeger, Phys. Rev. B: Condens. Matter Mater. Phys., 1980, 22, 2099.

14 S. Brazovskii and N. Kirova, Chem. Soc. Rev., 2010, 39, 2453.

15 C. S. Pinheiro and G. M. e Silva, Phys. Rev. B: Condens. Matter Mater. Phys., 2002, 65, 094304.

16 S. Stafström, Chem. Soc. Rev., 2010, 39, 2484.

17 Z. G. Yu, M. W. Wu, X. S. Rao and A. R. Bishop, J. Phys.: Condens. Matter, 1996, 8, 8847.

18 Z. Sun, Y. Li, K. Gao, D. Liu, Z. An and S. Xie, Org. Electron., 2010, 11, 279.

19 Z. Sun and S. Stafstöm, J. Chem. Phys., 2011, 134, 044906.

20 P. H. O. Neto, W. F. da Cunha, J. F. Teixeira, R. Gargano and G. M. e Silva, J. Phys. Chem. Lett., 2012, 3, 3039.

21 L. A. Ribeiro, W. F. da Cunha, P. H. O. Neto, R. Gargano and G. M. e Silva, Chem. Phys. Lett., 2013, 555, 168.

22 L. A. Ribeiro, W. F. da Cunha, P. H. O. Neto and G. M. e Silva, Phys. Procedia, 2012, 28, 112.

23 A. Johansson and S. Stafström, Phys. Rev. Lett., 2001, 86, 3602 .

24 A. Johansson and S. Stafström, Phys. Rev. B: Condens. Matter Mater. Phys., 2004, 69, 235205.

25 Z. An, C. Q. Wu and X. Sun, Phys. Rev. Lett., 2004, 93, 216407.

26 G. M. e Silva and A. Terai, Phys. Rev. B: Condens. Matter Mater. Phys., 1993, 47, 12568.

27 G. M. e Silva, Phys. Rev. B: Condens. Matter Mater. Phys., 2000, 61, 10777.

28 M. P. Lima and G. M. e Silva, Phys. Rev. B: Condens. Matter Mater. Phys., 2006, 74, 224303.

29 J. M. W. R. D. S. J. A. Izaguaire and D. P. Catarello, J. Chem. Phys., 2001, 114, 2090.

30 G. Cerullo, G. Lanzani, M. Z. S. S. de Silvestri, S. D. Comoretto, I. Moggio and G. Dellepiane, Synth. Met., 2001, 116, 57.

31 L. A. Ribeiro, P. H. O. Neto, W. F. da Cunha, L. F. Roncaratti, R. Gargano, D. A. da Silva Filho and G. M. e Silva, J. Chem. Phys., 2011, 135, 224901.

32 L. A. Ribeiro, W. F. da Cunha, P. H. O. Neto, R. Gargano and G. M. e Silva, Chem. Phys. Lett., 2013, 580, 108.

33 L. A. Ribeiro, W. F. da Cunha, P. H. O. Neto, R. Gargano and G. M. e Silva, New J. Chem., 2013, 37, 2829.

34 Z. Sun and S. Stafstöm, J. Chem. Phys., 2013, 138, 164905.

35 P. H. O. Neto, W. F. da Cunha, L. F. Roncaratti, R. Gargano and G. M. e Silva, Chem. Phys. Lett., 2010, 493, 283. 
View Article Online

PCCP

Paper

36 L. F. Roncaratti, R. Gargano, P. H. de Oliveira Neto, W. F. da Cunha, D. A. da Silva Filho and G. M. e Silva, Chem. Phys. Lett., 2012, 593, 214.

37 L. F. Roncaratti, R. Gargano and G. M. e Silva, J. Phys. Chem. A, 2009, 113, 14591.

38 C. Q. Wu, Phys. Rev. B: Condens. Matter Mater. Phys., 1993, 113, 4204.

39 H. Zhao, Y.-G. Chen, X.-M. Zhang, Z. An and C.-Q. Wu, J. Chem. Phys., 2009, 130, 234908.

40 Y. Meng, B. Di, X. J. Liu, Z. An and C. Q. Wu, J. Chem. Phys., 2008, 128, 184903.

41 Y. Meng, B. Di, X. J. Liu, Z. An and C. Q. Wu, Europhys. Lett., 2007, 79, 17002.

42 H. Ma and U. Schollwöck, J. Chem. Phys., 2008, 129, 244705.

43 H. Ma and U. Schollwöck, J. Phys. Chem. A, 2010, 114, 5439.

44 H. Ma and U. Schollwöck, J. Phys. Chem. A, 2009, 113, 1360.

45 Y. L. Zhang, X. J. Liu, Z. Sun and Z. An, J. Phys. Chem. C, 2014, 118, 2963.

46 Y. L. Zhang, X. J. Liu, Z. Sun and Z. An, J. Chem. Phys., 2013, 138, 174906.

47 Z. Sun, Y. Li, S. J. Xie, Z. An and D. S. Liu, Phys. Rev. B: Condens. Matter Mater. Phys., 2014, 79, 201310(R). 


\section{ApPENDIX J PAPER X}

\section{Temperature Effects on Intrachain Recombination of Bipolarons in Conjugated Polymers}

Luiz Antonio Ribeiro Junior Wiliam Ferreira da Cunha Antonio Luciano de Almeida Fonseca Ricardo Gargano Geraldo Magela e Silva

Chem. Phys. Lett. 614, 151-155, (2014). 


\title{
Temperature effects on intrachain recombination of bipolarons in conjugated polymers
}

\author{
Luiz Antonio Ribeiro Junior ${ }^{a}$, Wiliam Ferreira da Cunha ${ }^{c}$, \\ Antonio Luciano de Almeida Fonseca ${ }^{\mathrm{c}}$, Ricardo Gargano ${ }^{\mathrm{b}, *}$, \\ Geraldo Magela e Silva ${ }^{\mathrm{C}}$
}

a Department of Physics, Chemistry and Biology (IFM), Linköping University, SE-581 83 Linköping, Sweden

${ }^{b}$ Departments of Chemistry and Physics, University of Florida, Quantum Theory Project, Gainesville, FL, 32611, USA

' Institute of Physics, University of Brasilia, 70.919-970, Brasilia, Brazil

A R T I C L E I N F O

Article history:

Received 23 July 2014

In final form 12 September 2014

Available online 19 September 2014

\begin{abstract}
A B S T R A C T
The temperature influence over the intrachain recombination dynamics between oppositely charged bipolarons has been theoretically investigated using a version of the Su-Schrieffer-Heeger model. The results shown that, below of a critical temperature regime, these charge carriers can recombine into a biexciton. This excited state is a light emissive specie that can emit one photon and turn into an exciton state. This specie can thereafter decay to the ground state by other photon emission. This knowledge can enlighten the understanding needed to improve the internal quantum efficiency of electroluminescence in Polymer Light Emitting Diodes.
\end{abstract}

(c) 2014 Elsevier B.V. All rights reserved.

\section{Introduction}

Since the discovery of poly(paraphenylene vinylene) (PPV) and its electroluminescence properties, there has been intensive efforts to understand the physical processes necessary to improve the efficiency of Polymer Light Emitting Diodes (PLEDs) [1]. Considerable quantities of theoretical studies, focusing on this objective, have been performed by finding channels for the recombination between polarons [2-4], bipolarons [5,6], polarons and bipolarons [6], polarons and excitons [7], and bipolarons and excitons [8]. Nevertheless, studies considering the temperature influence on such processes remains lacking both theoretically and also experimentally.

Bipolarons can be created in PLEDs when the charge injection results in a considerable concentration of polarons. Two acoustic polarons with the same charge and antiparallel spins, for example, can recombine in order to form an acoustic bipolaron. In these devices, the generation of excited states is a fundamental physical process. Particularly, it is believed that bipolaron recombination is one of the key steps behind the mechanism of electroluminescence in PLEDs. Recently, Di and coworkers used

\footnotetext{
* Corresponding author.

E-mail addresses: luiju@ifm.liu.se (L.A. Ribeiro Junior), gargano@unb.br, gargano@fis.unb.br (R. Gargano).
}

the Su-Schrieffer-Heeger (SSH) and the extended Hubbard models to simulate the bipolaron recombination process in conjugated polymers [5]. Their results show that two bipolarons can scatter into singlet biexciton states in both mono-layer and multiplelayer electroluminescence polymeric materials. Sun and Stafström have simulated the same process depicting the effects of the electron-electron interactions for a system composed by two coupled conjugated polymer chains [9]. Their results indicate that there are four channels for the bipolaron recombination: (1) forming a biexciton, (2) forming an excited negative polaron and a free hole,

(3) forming an excited positive polaron and a free electron, and (4) forming an exciton, a free electron and a free hole. Our recent previous research has also shown that the presence of impurities in a conjugated polymer lattice, for several electric field regimes, favors the excited states formation and also improves the excitation yields when the scattering between an oppositely charged bipolarons is considered [6]. There are, however, still many controversial aspects regarding the influence of physical process such as electric field strength, electron-electron interactions, temperature, and impurity effects on the formation of excited states via collision process between oppositely charged carriers, which claims better phenomenological descriptions.

In this contribution, a systematic numerical investigation considering the influence of an external electric field, Coulomb interactions, and temperature effects on the formation of excited states via recombination process between an oppositely charged 
bipolaron pair is performed using a cis-polyacetylene chain. These excited states (biexcitons) are very important due to the fact that they can decay to an exciton, which can subsequently decay to the ground state, emitting two photons throughout the process. In this way, the understanding of these important processes may provide guidance to improve electroluminescence yields in PLDEs.

\section{Model and method}

The model Hamiltonian is given by $H=H_{S S H}+H_{e e} . H_{S S H}$ is the SSH-type Hamiltonian $[10,11]$ that assumes the following form:

$H_{S S H}=-\sum_{n, s}\left(t_{n, n+1} C_{n+1, s}^{\dagger} C_{n, s}+\right.$ h.c. $)+\sum_{n} \frac{K}{2} y_{n}^{2}+\sum_{n} \frac{p_{n}^{2}}{2 M}$,

where $n$ indexes the sites of the chain. In the classical part of $H_{S S H}$ (lattice backbone) $K$ represents the harmonic constant that describes a $\sigma$ bond, $M$ is the mass of a $C H$ group, and $p_{n}$ is the conjugated momentum to $u_{n}$, which is the lattice displacement of an atom at the $n$th site. Considering the electronic part of $H_{S S H}$, the operator $C_{n, S}^{\dagger}\left(C_{n, S}\right)$ creates (annihilates) a $\pi$ electron state at the $n$th site with spin $s, t_{n, n+1}$ is the transfer integral [12], given by $t_{n, n+1}=e^{-i \gamma A(t)}\left[\left(1+(-1)^{n} \delta_{0}\right) t_{0}-\alpha y_{n}\right]$, where $y_{n}$ is defined as $y_{n} \equiv u_{n+1}-u_{n}, t_{0}$ is the transfer integral of a $\pi$-electron between nearest neighbor sites in the undimerized chain, $\alpha$ is the electron-phonon coupling constant, and $\delta_{0}$ is the Brazovskii-Kirova-type symmetry-breaking term. In the transfer integral expression $\gamma \equiv e a /(\hbar c)$ with $e$ being the absolute value of the electronic charge, $a$ is the lattice constant, and $c$ is the speed of light. The electric field is included in the Hamiltonian as a scalar potential $A$ having the time dependence in the form of a half Gaussian $[13,14]$.

The term $H_{e e}$ in the model Hamiltonian denotes the contribution of the electron-electron interactions and can be written as

$$
\begin{aligned}
H_{e e}= & U \sum_{i}\left(C_{i, \uparrow}^{\dagger} C_{i, \uparrow}-\frac{1}{2}\right)\left(C_{i, \downarrow}^{\dagger} C_{i, \downarrow}-\frac{1}{2}\right) \\
& +V \sum_{i}\left(n_{i}-1\right)\left(n_{i+1}-1\right),
\end{aligned}
$$

where $U$ is the on-site and $V$ the nearest-neighbor Coulomb repulsion strengths and $n_{i}=C_{i, \uparrow}^{\dagger} C_{i, \uparrow}+C_{i, \downarrow}^{\dagger} C_{i, \downarrow}$ [15]. The parameters used here are $t_{0}=2.5 \mathrm{eV}, M=1349.14 \mathrm{eV} \times \mathrm{fs}^{2} / \AA^{2}, K=21 \mathrm{eV} \AA^{-2}, \delta_{0}=0.05$, $\alpha=4.1 \mathrm{eV} \AA^{-1}, a=1.22 \AA$, and a bare optical phonon energy $\hbar \omega_{Q}=$ $\hbar \sqrt{4 K / M}=0.16 \mathrm{eV}$. These values have been used successfully in previous simulations [16-22].

The lattice backbone dynamics is carried out by means of the Euler-Lagrange equations, that lead to a Newtonian equation

$$
\begin{aligned}
F_{n}(t)= & M \ddot{u}_{n}(t) \\
= & -K\left[2 u_{n}(t)-u_{n+1}(t)-u_{n-1}(t)\right] \\
& +\alpha\left[B_{n, n+1}(t)-B_{n-1, n}(t)+B_{n+1, n}(t)-B_{n, n-1}(t)\right],
\end{aligned}
$$

where, $F_{n}(t)$ represents the force on the $n$th site. Here, the term $B_{n, n^{\prime}}(t)=\sum_{k, s}^{\prime} \psi_{k, s}^{*}(n, t) \psi_{k, s}\left(n^{\prime}, t\right)$ couples the electronic and lattice solutions [23]. The primed summation represents a sum over occupied states. $F_{n}(t)$ can be numerically integrated using the method $u_{n}\left(t_{j+1}\right)=u_{n}\left(t_{j}\right)+\dot{u}_{n}\left(t_{j}\right) \Delta t$ and $\dot{u}_{n}\left(t_{j+1}\right)=\dot{u}_{n}\left(t_{j}\right)+$ $\left(F_{n}\left(t_{j}\right) / M\right) \Delta t$. The time dependent wave functions are constructed using a linear combination of instantaneous eigenstates of the electronic part of $H_{S S H}$. The solutions of the time-dependent Scrhödinger equation can be written in the form

$$
\begin{aligned}
\psi_{k, s}\left(n, t_{j+1}\right)= & \sum_{l}\left[\sum_{m} \phi_{l, s}^{*}\left(m, t_{j}\right) \psi_{k, s}\left(m, t_{j}\right)\right] \\
& \times e^{\left(-i \varepsilon_{l} \Delta t / \hbar\right)} \phi_{l, s}\left(n, t_{j}\right) .
\end{aligned}
$$

$\left\{\phi_{l}(n)\right\}$ and $\left\{\varepsilon_{l}\right\}$ are the eigenfunctions and the eigenvalues of the electronic part of $H_{S S H}$ at a given time $t_{j}$ [24]. Here, the temperature effects are simulated as detailed in one of our recent researches [3]. It should be emphasized that this procedure of including thermal effects by means of a Langevin Equation has been extensively used in the literature with a good track record [14,25-32].

\section{Results}

The recombination dynamics between the oppositely charged bipolaron pair is performed in systems composed of 200-site cispolyacetylene chains with periodic boundary conditions. For the electric field, turned on quasi-adiabatically [14], the values used in the simulations varied from 0.5 to $2.0 \mathrm{mV} / \AA$ with an increment of $0.5 \mathrm{mV} / \AA$, whereas the temperature regimes considered ranged from 50 to $300 \mathrm{~K}$ with a step of $25 \mathrm{~K}$. The on-site and nearestneighbor electron-electron interactions considered are $U=0.2 t_{0}$ and $V=U / 2$, respectively. In this context, Figure 1 presents the schematic diagram of energy levels for a lattice containing a hole-bipolaron (positive carrier) and an electron-bipolaron (negative carrier). These charge carriers are formed removing or adding electrons to the polymer chain, causing lattice distortions and the rising of energy levels to the inside of the band gap. A single polymer chain containing the hole-bipolaron, is represented by the absence of two electrons in the HOMO- 1 and LUMO+1 levels, which yields $\mathrm{a}+2 \mathrm{e}$ charge. On the other hand, an electron-bipolaron is represented by the levels HOMO and LUMO, in which both levels are occupied by two electrons, thus yielding a $-2 e$ net charge.

We start the discussion presenting the biexciton formation due to the bipolaron recombination under a temperature regime of $150 \mathrm{~K}$ and an electric field strength of $1.5 \mathrm{mV} / \AA$, as shown in Figure 2. The temporal evolution of the staggered bond-length $\bar{y}(t)=(-1)^{n}\left[y_{n-1}(t)+2 y_{n}(t)+y_{n+1}(t)\right] / 4$, that depicts the dynamical process where an biexcitonic state is formed directly after the recombination between the bipolarons, is presented in Figure 2(a). Initially, the electron-bipolaron is located at the 60th site while the hole-bipolaron is at 150th site. The two charge carriers are

\section{CONDUCTION BAND}

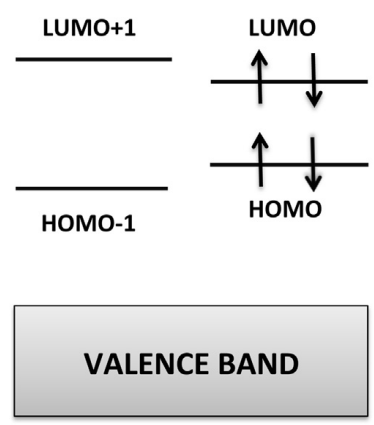

Figure 1. The schematic diagram of energy levels for a polymer chain containing an electron-bipolaron and a hole-bipolaron. 

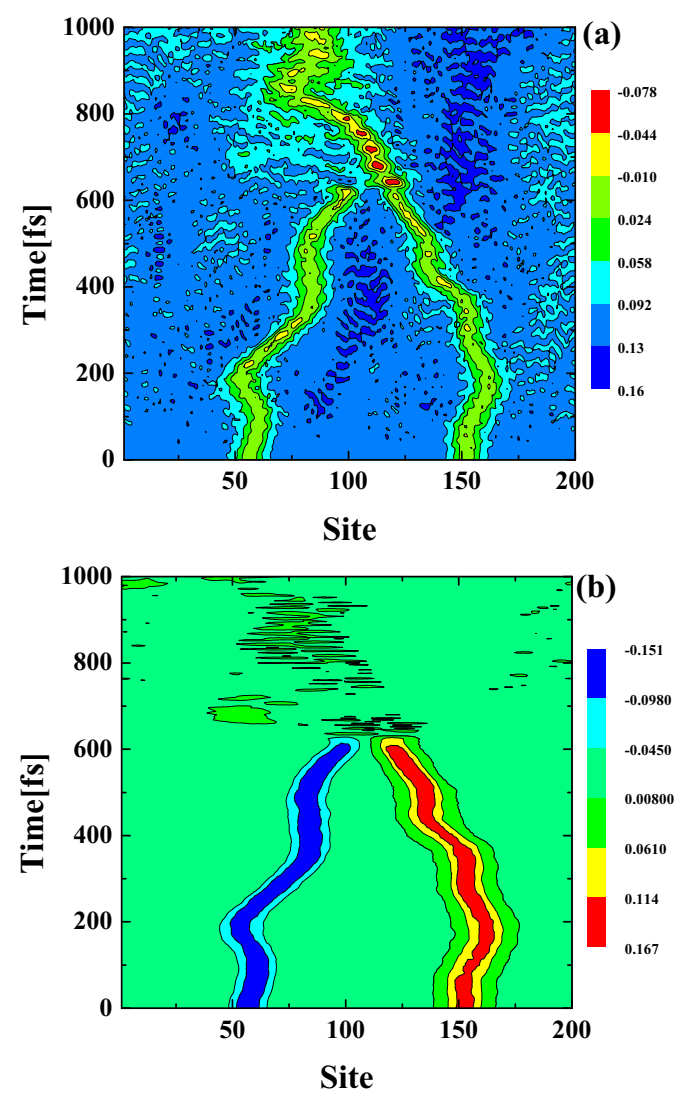

Figure 2. (a) Staggered bond-length and (b) mean charge density time evolution for an electric field strength of $1.5 \mathrm{mV} / \AA$ and temperature regime of $150 \mathrm{~K}$.

separated far enough from one another so that they behave as independent structures. After a small transient time for the electric field response, the bipolarons begin to move toward each other due to their opposite charges. However, at approximately $50 \mathrm{fs}$ the adding up of thermal energy begins to become considerable and the bipolarons start to perform a random walk along the chain influenced by the thermal random forces. The bipolaron dynamics before the collisional processes occurs as described in the literature $[13,19]$. At around $600 \mathrm{fs}$, when the bipolarons begin to interact, one can see that they quickly recombine into a well localized structure, yielding the formation of only one neutral excited state. In this process, their wave functions begin to overlap strongly and, as a result of the Coulomb interactions, the two bipolarons are attracted to each other loosing their kinetic energy acquired from the electric field. The Coulomb interactions together with the self-trapped state, produce a singlet biexciton deformation by transferring two electrons from the negative bipolaron to the positive one. Also, it can be seen that after about $100 \mathrm{fs}$, the adding up of thermal energy begins to become visible through the blurring of the figure. Another important aspect can be noted in Figure 2(a) comparing the lattice deformation for the biexciton with the lattice defect that characterizes a bipolaron. The lattice distortion for the biexciton is wider than that for the bipolaron, which shows that the distortion for neutral excitations involve a higher number of sites. Also, the red regions, not present in the bipolaron lattice defects, indicates a higher level in deformation of the lattice, which suggests that biexcitons are structures as stable as bipolarons.
The results of this process can be further clarified by analyzing the temporal evolution of the mean charge density $\bar{\rho}(t)=$ $1-\left[\rho_{n-1}(t)+2 \rho_{n}(t)+\rho_{n+1}(t)\right] / 4$ before and after the recombination, as shown in Figure 2(b). Immediately after the bipolarons collision, their charges cancel each other. It can be conjectured that this charge cancellation process occurs due to the excited state formation. Figure 2(b) also shows that there is a slight net charge coupled to the biexciton lattice deformation that is induced by the lattice fluctuations caused by thermal random forces. This channel for the bipolaron pair recombination is only observed for electric fields strengths between 1.5 and $2.0 \mathrm{mV} / \AA$ and temperature regimes smaller than $200 \mathrm{~K}$. For temperature higher than this critical value, the bipolaron motion is damped avoiding the interaction between the charge carriers. The lattice fluctuations reach such high amplitudes that the bipolarons are annihilated in the collision. This situation is similar to the one proposed in one of our recent works [6]. Furthermore, the system presented in Figure 2(b) yields an neutral excited state with a better rate than those previously reported in this previous research, where exactly the same kind of calculations were performed in absence of temperature effects. In this study it was obtained that, for electric field strengths between 1.0 and $2.0 \mathrm{mV} / \AA$, an inelastic scattering processes between oppositely charged bipolarons into an mixed state composed of bipolarons and excitons took place. Considering the temperature effects over the system, the same behavior is obtained in the present work for electric fields strengths between 1.0 and $1.5 \mathrm{mV} / \AA$ a and temperature regimes smaller than $150 \mathrm{~K}$. It is believed that the lattice fluctuations and the high energy of phonons provided by the inclusion of thermal effects - absent in results reported in reference [6] - are responsible for this yield for the biexciton formation.

In order to better understand the properties of the localized excitation formed after the recombination, it is useful to discuss the changes of the electronic structure of the system in the processes by means of the time evolution of the intra-gap levels and their occupation numbers [25]. During the transient regime (from 0 to $600 \mathrm{fs}$ ), the occupation number of the LUMO (HOMO) level sharply decreases from 2 to 0 whereas the occupation number of LUMO+1 (HOMO-1) sharply increases from 0 to 2 . This change in occupation number reflects the fact that these two levels interchange positions. Particularly, the electron transfer associated with this change occurs due to the fact that the wavefunction associated with this level spreads over the whole chain. After $600 \mathrm{fs}$, the nondegeneracy among the levels becomes significant by the interplay between electric field, the electron-electron interactions, and by the coupling between the two bipolarons when they starts to merge. From that moment, the interchange between the positions of the energy levels is no longer observed. The electronic transfer between the levels that leads to the biexciton production can be followed by analyzing the time evolution of the occupation numbers as shown in Figure 3. This figure shows the time evolution of the occupation numbers for the intra-gap levels related to Figure 1. After a transient period in which the occupation number oscillates due to the interaction, it is possible to note that the final state in the LUMO+1 level is covered by almost two electrons, whereas the occupation for the LUMO level falls dramatically close zero after roughly $700 \mathrm{fs}$. Also, the small remaining fraction of the LUMO level, which is not transferred to LUMO+1, decays to the HOMO level resulting in an photon emission process. Analogously to the occupation process for the LUMO+1, the HOMO level receives the electron transferred from the HOMO-1. However, in this case, only one electron is transferred among these levels. This process depicts an interesting behavior regarding the biexciton states structure in which such excited states can be formed by a suitable balance in the transfer of three electrons between the intra-gap levels. The final configuration for the occupation numbers reported here results 


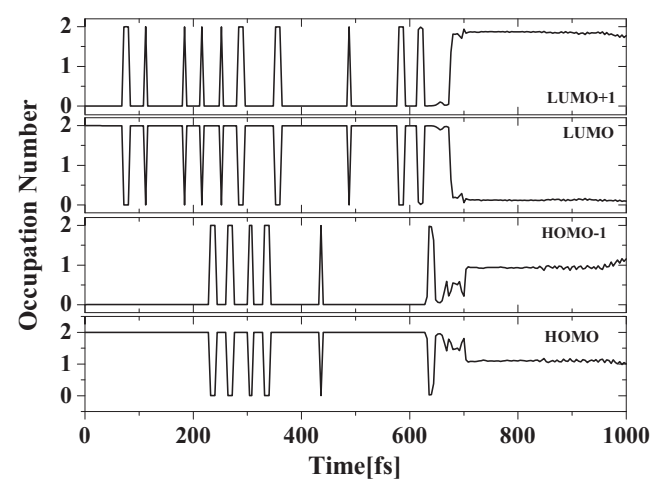

Figure 3. Time evolution of the occupation number.

in a lattice where a bipolaron defect is absent and only one well defined deformation is observed after the recombination process. In this way, these results indicate that, in general, mechanisms such as electron transfer among the levels and photon emission will induce the formation of biexciton states by two means: from a bipolaron recombination process at temperature regimes below of $150 \mathrm{~K}$. Otherwise a dimerized lattice (similar to the case reported in reference [6]) will take place for temperatures higher than this critical value, considering useful electric field strengths for technologic applications in conjugated polymers between 1.0 and $2.0 \mathrm{mV} / \AA$.

While the occupation number analysis is the most suitable tool in studying the process that leads to the formation of new species such as excited states, the stability of the quasi-particles is better described by the time evolution analysis of the energy levels. We finish our discussion by presenting in Figure 4 the time evolution profile of the intra-gap energy levels of the simulations shown in Figure 2. As can be seen from Figure 4, at the beginning there are four intra-gap levels, which are localized electron states caused by the presence of the bipolarons in the lattice. According to their wave functions, we know that the purple and red lines correspond to the positive bipolaron levels HOMO-1 and LUMO, while the green and blue levels are related to the negative bipolaron levels HOMO and LUMO+1, respectively. At the beginning of the simulation the degenerate levels become nondegenerated due to the temperature influence and the electron-electron interactions. The recombination process between the bipolaron pair is noted to take place at approximately $650 \mathrm{fs}$, when the LUMO and HOMO- 1 levels move to the conduction and valence band respectively, whereas

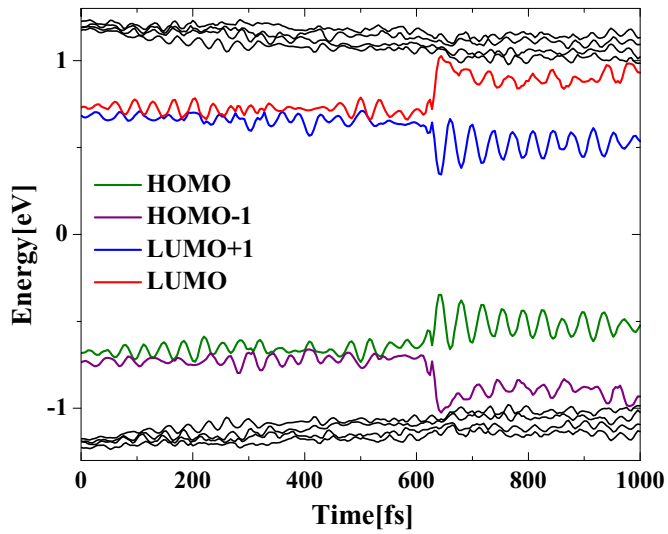

Figure 4. Time evolution of energy levels at the gap. the HOMO and LUMO+1 levels move towards the midgap region. Also, the resulting phonons produced from the recombination process are represented by the deeper oscillations of the energy levels inside the band gap. The final configuration of the energy levels shows a physical picture where both bipolarons are annihilated and one biexciton arises after the interaction between the original charge carriers. Still according to their wave functions, we know that the biexciton energy levels present a deeper narrowing when compared to those of the bipolaron. This fact suggests that the biexcitons structure are more stable than bipolarons. Furthermore, the oscillations presented by the energy levels in all the process are resulting from the motion of the carbons sites of the lattice influenced by the thermal random forces.

Finally, it is worth to mention some important aspects regarding the light-emissive characteristic of the biexciton. This neutral excited structure is represented by two intra-gap localized states (as shown in Figure 4), in which the upper one is doubly occupied whereas the lower one lies empty. The wavefunctions associated with these localized energy levels have opposite parity. Theoretical investigations have shown that the radiative process BIEXCITON $\rightarrow$ EXCITON $+h v$ has large transition dipole moments (8.8 eÅ). In this way, the biexcitonic state can decay to an exciton state by one photon emission [5]. Subsequently the emission, the biexciton becomes a singlet exciton. The resulting excitonic state has also two localized intra-gap energy levels with opposite parity, where these levels are now occupied by one electron with opposite spin. Moreover, the results have indicated that the radiative process EXCITON $\rightarrow$ GROUND STATE $+h v$ also has large transition dipole moments ( $5.5 \mathrm{e} \AA$ ), which are the same order of magnitude as those of the biexciton. After that, the exciton state can decay to the ground state by emitting one photon.

\section{Conclusions}

In summary, using a tight-binding electron-phonon interaction model modified to include temperature effects, external electric field, and electron-electron interactions, the recombination processes between an electron-bipolaron and a hole-bipolaron has been simulated in a conjugated polymer lattice. The results show that, depending on the considered situation, there are two different channels resulting from the recombination between charged bipolarons. For a given regime, there exists a critical temperature value, below which a biexcitonic state is directly formed. Differently, a dimerized lattice is the resulting product of the collisional process, when the temperature is higher than the critical value. Furthermore, it is found that both channels depend sensitively on the strength of the applied electric field. Obtaining such different regimes and the critical temperature values needed to reach one or the other channel is crucial to fully control the charge carrier density in conductive polymers. These significant results reveal remarkable details concerning the bipolaron recombination mechanism and provide guidance to understanding electroluminescence process in Polymer Light Emitting Diodes.

\section{Acknowledgements}

The authors gratefully acknowledge the Swedish Research Council (VR) for financial support, the Swedish National Infrastructure for Computing (SNIC), and the and the financial support from the Brazilian Research Councils CNPq, CAPES and FINATEC.

\section{References}

[1] M.S. White, et al., Nat. Phot. 7 (2013) 811.

[2] Z. An, B. Di, C.Q. Wu, Eur. Phys. J. B 63 (2008) 71. 
[3] W.F. da Cunha, L.A.R. Junior, R. Gargano, G.M. e Silva, Phys. Chem. Chem. Phys, 16 (2014) $17072-17080$

[4] L.A. Ribeiro, W.F. da Cunha, P.H.O. Neto, R. Gargano, G.M. e Silva, J. Chem. Phys. 139 (2013) 174903

[5] B. Di, Y. Meng, Y.D. Wang, X.J. Liu, Z. An, J. Chem. Phys. B 115 (2011) 9339

[6] L.A. Ribeiro, W.F. da Cunha, P.H. de Oliveira Neto, R. Gargano, G.M. e Silva, J. Phys. Chem. B 117 (39) (2013) 11801.

[7] Z. Sun, D. Liu, S. Stafstöm, Z. An, J. Chem. Phys. 134 (2011) 044906.

[8] Z. Sun, Y. Li, D.S. Li, Z. An, S. Xie, Phys. Rev. B 134 (2009) 201310 (R).

[9] Z. Sun, S. Stafström, J. Chem. Phys. 135 (2011) 074902

[10] W.P. Su, J.R. Schrieffer, A.J. Heeger, Phys. Rev. Lett. 42 (1979) 1698.

[11] W.P. Su, J.R. Schrieffer, A.J. Heeger, Phys. Rev. B 22 (1980) 2099

[12] C.S. Pinheiro, G.M. e Silva, Phys. Rev. B 65 (2002) 094304

[13] S. Stafström, Chem. Soc. Rev. 39 (2010) 2484.

14] L.A. Ribeiro, W.F. da Cunha, P.H.O. Neto, R. Gargano, G.M. e Silva, N. J. Chem. 37 (2013) 2829.

[15] Z.G. Yu, M.W. Wu, X.S. Rao, A.R. Bishop, J. Phys.: Condens. Matter 8 (1996) 8847 [16] P.H.O. Neto, W.F. da Cunha, J.F. Teixeira, R. Gargano, G.M. e Silva, J. Phys. Chem. Lett. 3 (2012) 3039

[17] L.A. Ribeiro, W.F. da Cunha, P.H.O. Neto, R. Gargano, G.M. e Silva, Chem. Phys. Lett. 555 (2013) 168.
[18] L.A. Ribeiro, W.F. da Cunha, P.H.O. Neto, G.M. e Silva, Phys. Proc. 28 (2012) 112

[19] A. Johansson, S. Stafström, Phys. Rev. Lett. 86 (2001) 3602.

20] A. Johansson, S. Stafström, Phys. Rev. B 69 (2004) 235205.

[21] Z. An, C. Wu, X. Sun, Phys. Rev. Lett. 93 (2004) 216407.

[22] G.M. e Silva, A. Terai, Phys. Rev. B 47 (1993) 12568.

[23] G.M. e Silva, Phys. Rev. B 61 (2000) 10777.

[24] M.P. Lima, G.M. e Silva, Phys. Rev. B 74 (2006) 224303.

[25] L.A. Ribeiro, P.H.O. Neto, W.F. da Cunha, L.F. Roncaratti, R. Gargano, D.A. da Silva Filho, G.M. e Silva, J. Chem. Phys. 135 (2011) 224901.

[26] L.A. Ribeiro, W.F. da Cunha, P.H.O. Neto, R. Gargano, G.M. e Silva, Chem. Phys. Lett. 580 (2013) 108.

[27] Z. Sun, S. Stafstöm, J. Chem. Phys. 138 (2013) 164905

[28] P.H.O. Neto, W.F. da Cunha, L.F. Roncaratti, R. Gargano, G.M. e Silva, Chem. Phys. Lett. 493 (2010) 283.

[29] L.F. Roncaratti, R. Gargano, P.H. de Oliveira Neto, W.F. da Cunha, D.A. da Silva Filho, G.M. e Silva, Chem. Phys. Lett. 593 (2012) 214

30] L.F. Roncaratti, R. Gargano, G.M. e Silva, J. Phys. Chem. A 113 (2009) 14591.

[31] L.A. Ribeiro Junior, W.F. da Cunha, G.M. e Silva, J. Phys. Chem. B 118 (19) (2014) 5250.

[32] Y. Zhang, X. Liu, Z. An, J. Chem. Phys. C 118 (6) (2014) 2963. 


\title{
APPBDOKK $\mathrm{K}$ \\ PAPER XI
}

\section{Reactive Scattering Between Excitons and Charge Carriers in Conjugated Polymers}

\author{
Wiliam Ferreira da Cunha \\ Luiz Antonio Ribeiro Junior \\ Antonio Luciano de Almeida Fonseca \\ Ricardo Gargano \\ Geraldo Magela e Silva
}

J. Phys. Chem. C., 118, 23451-23458, 2014. 


\title{
Reactive Scattering between Excitons and Charge Carriers in Conjugated Polymers
}

\author{
Wiliam Ferreira da Cunha, ${ }^{* \dagger}{ }^{\dagger}$ Luiz Antonio Ribeiro, Jr., ${ }^{*}, \dot{\dagger}$ Antonio Luciano de Almeida Fonseca, ${ }^{\dagger}$ \\ Ricardo Gargano, ${ }^{\dagger}$ and Geraldo Magela e Silva ${ }^{\dagger}$ \\ ${ }^{\dagger}$ Institute of Physics, University of Brasilia, 70.919-970, Brasilia, Brazil \\ ${ }^{*}$ Department of Physics, Chemistry and Biology (IFM), Linköping University, SE-581 83 Linköping, Sweden
}

ABSTRACT: The temperature influence on the scattering process between an exciton and a positively charged carrier (polaron or bipolaron) is theoretically investigated using a version of the $\mathrm{Su}-\mathrm{Sch}$ rieffer-Heeger (SSH) model modified to include temperature, Coulomb interactions, and an external electric field. In general, it is observed that the products of the reactive scattering are spin independent when thermal effects are taken into account. For the interaction between a polaron and an exciton, the polaron can be annihilated, when subjected to temperatures higher than a critical value, or pass through

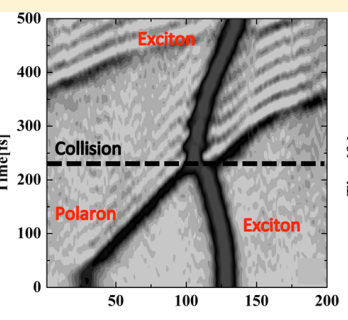

Sit

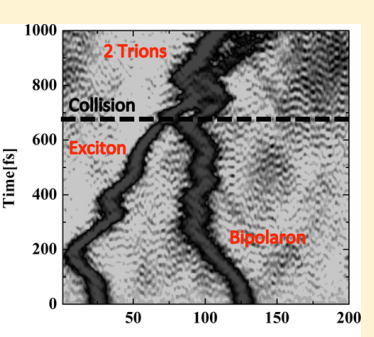

Site temperature regime is considered. Regarding the recombination between a bipolaron and an exciton, it is observed that the bipolaron can be annihilated whereas the exciton dissociates into two trions or into one polaron and one trion. In all cases, the recombination mechanisms depend on a suitable balance between temperature and electric field. These results may extend the knowledge about electroluminescence process in conjugated polymers, thus being of potential use to improve internal quantum efficiency in polymer light-emitting diodes.

\section{INTRODUCTION}

Conjugated polymers have attracted considerable interest as ideal candidates for the development of optoelectronic devices such as polymer light-emitting diodes (PLEDs). Their optoelectronic features combined with potential advantages in terms of ease of synthesis, flexibility, low cost, and large-area capability make them more attractive materials for the electronics industry than their inorganic counterparts to provide new display technologies. Furthermore, since the discovery of poly ( $p$-phenylenevinylene) (PPV) and its electroluminescence properties, there has been intensive efforts to understand the physical processes necessary to improve the efficiency of PLEDs. ${ }^{1,2}$ A considerable amount of theoretical studies has been performed by finding channels for the recombination between polarons, ${ }^{3-8}$ bipolarons, ${ }^{9,10}$ polarons and bipolarons, ${ }^{11}$ polarons and excitons, ${ }^{5}$ and bipolarons and excitons. ${ }^{12}$ Nevertheless, studies considering the temperature influence on such processes, although crucial to a more realistic description of the system, remain not available theoretically and are also very difficult to control experimentally.

In conjugated polymers, the usual self-localized charge carriers are polarons, which possess spin $1 / 2$ and charge $\pm e$. On the other hand, bipolarons are spinless structures with charge $\pm 2 e$ that, similarly to polarons, are generated upon doping or photoexcitation. Some experimental and theoretical studies have shown the existence of bipolarons mainly in doped polymer layers. ${ }^{13-17}$ Bipolarons can be created in PLEDs when the charge injection results in a large concentration of polarons.
For example, two acoustic polarons with the same charge and antiparallel spins can recombine to form an acoustic bipolaron. ${ }^{9}$ Another important excitation in the context of PLEDs is the exciton. Analogously to the conventional charge carriers in these materials, exciton are also structures associated with the strong coupling between charge and phonons and it is linked to a structural deformation. An exciton is created via absorption of a photon but can also be the result of the interaction between two polarons with opposite charges, ${ }^{4,8}$ thus resulting in zero net charge. Since polarons, bipolarons, and excitons coexist in PLEDs, there exists a high possibility of collision between these excitations in order to form new excited states. Studies concerning the interaction between charge carriers and excitons can provide a deeper understanding of the properties of conjugated polymers in general. Particularly, it is believed that the generation of excited states is one of the key steps behind the mechanism of electroluminescence in PLEDs.

It has been conjectured that when charge carriers and excitons overlap in space, they can collide and recombine to form excited species. The photon emission phenomena depends directly on the radiative decay of such species. Recently, Sun and colleagues studied the scattering processes of a bipolaron with an exciton reporting the products formed from this reaction using the $\mathrm{SSH}$ method. ${ }^{12}$ It was identified one

Received: May 12, 2014

Revised: September 25, 2014

Published: September 29, 2014 
channel for the formation of excited species, where a polaron and an excited polaron are created. Because of relaxation of the excited polaron, the exciton is annihilated and the bipolaron dissociates into two polarons. In other investigations performed also by Sun and collaborators, the scattering process between a polaron and an exciton, considering two composite spin states, is simulated in a polymer chain using the same approach. ${ }^{18}$ For one of the composite spin state, the repulsion between the polaron and the exciton is very weak. Therefore, the polaron can easily pass through the exciton even in weak external electric field. For the other composite spin state, the repulsion between the polaron and the exciton is much stronger. In this case the polaron may either be bounced back, be dissociated, or pass through the exciton, depending on the strength of the external electric field. However, there are still many controversial aspects regarding the influence of some physical process such as temperature, Coulomb interactions, composite spin configuration, and impurity effects on the formation of excited states via recombination process between oppositely charged carriers and excitons. Thus, these features require detailed phenomenological descriptions.

In this paper, a systematic numerical investigation considering the influence of an external electric field, Coulomb interactions, and temperature effects on the formation of excited states via recombination process between charge carriers and excitons is performed using a cis-polyacetylene chain in the scope of a nonadiabatic evolution method. An Ehrenfest molecular dynamics is carried out by using a onedimensional tight-binding model including lattice relaxation. Combined with the extended Hubbard model (EHM), a modified version of the SSH model is used to include an external electric field and the Brazoviskii-Kirova symmetry breaking term. Temperature effects are included by means of a canonical Langevin equation. The aim of this work is to give a physical picture of the excited states formation due to the recombination of charge carriers and excitons in conjugated polymers, particularly when temperature effects are considered. The excited states formed through this mechanisms can decay to the ground state, emitting photons after the recombination reaction. In this way, the understanding of these important processes may provide guidance to improve electroluminescence yields in PLDEs.

\section{MODEL AND METHOD}

In this work we considered a polyacetylene chain in the cis configuration in order to investigate temperature effects on the generation of excited states in organic semiconductors. As the SSH scheme consists on a semiempirical tight binding with lattice relaxation in a first-order expansion, our results are expected to be valid for conjugated polymers in general, provided an appropriated parametrization is conducted. ${ }^{19-25}$ The complete Hamiltonian of the model is given by ${ }^{26,27}$

$$
\begin{aligned}
H & =-\sum_{n, s}\left(t_{n, n+1} C_{n+1, s}^{\dagger} C_{n, s}+\text { h. c. }\right)+\sum_{n} \frac{K}{2} y_{n}{ }^{2} \\
& +\sum_{n} \frac{p_{n}^{2}}{2 M}+U \sum_{n}\left(C_{n, \uparrow}^{\dagger} C_{n, \uparrow}-\frac{1}{2}\right)\left(C_{n, \downarrow}^{\dagger} C_{n, \downarrow}-\frac{1}{2}\right) \\
& +V \sum_{n}\left(m_{n}-1\right)\left(m_{n+1}-1\right)
\end{aligned}
$$

The fundamental variables of the model are the quantities $y_{n} \equiv$ $u_{n+1}-u_{n}$ and $p_{n}$. The former represents the sites displacement, and the latter is the momentum conjugated to $u_{n}$, in the scope of the Hamiltonian formalism. When compared to the original model, the hopping integral $t_{n, n+1}$ used in the present work is modified to $t_{n, n+1}=\mathrm{e}^{-i \gamma A t}\left[\left(1+(-1)^{n} \delta_{0}\right) t_{0}-\alpha y_{n}\right]$. This was carried out so that the electric field can be considered by means of the potential vector $\mathbf{A}(t)$ through a Peirls modification on the phase factor, ${ }^{29}$ and the symmetry breaking associated with the cis configuration can be included by the presence of the Brazovskii-Kirova term $\delta_{0}$. It should be emphasized the semiclassical nature of our Hamiltonian: whereas the first summation is expressed in terms of annihilation $\left(C_{n, s}\right)$ and creation $\left(C_{n, s}^{\dagger}\right)$ operators-which, in the spirit of the second quantization formalism annihilates and creates a spin " $\mathrm{s}$ " electron in the $n$th site, respectively-the second and third summation stand for a linear harmonic lattice with elastic constant $K$ and mass $M$. The separation between the classical and quantum realms, however, is not complete since the $\alpha$ constant couples the electronic and the lattice part of the system. For the present particular case of cis-polyacetylene we used the following parameters, considering $\gamma \equiv e a /(\hbar c), a$ the lattice constant, $e$ the electronic fundamental charge, and $c$ the speed of light: $t_{0}=2.5 \mathrm{eV}, M=1349.14 \mathrm{eV} \times \mathrm{fs}^{2} / \AA^{2}, K=21 \mathrm{eV}$ $\AA^{-2}, \delta_{0}=0.05, \alpha=4.1 \mathrm{eV} \AA^{-1}, a=1.22 \AA$, and a bare optical phonon energy $\hbar \omega_{Q}=\hbar(4 \mathrm{~K} / \mathrm{M})^{1 / 2}=0.16 \mathrm{eV}$.

Finally, we consider the Hartree-Fock approach to treat the electronic correlation. Several theoretical and experimental results have demonstrated that the primary excitation is the exciton, and electron-electron interactions are dominant over electron-lattice interactions in luminescent polymers. ${ }^{30-35}$ Some theoretical studies that have considered the electronlattice interactions also show that introduction of the lattice relaxation effect would not lead to an increase in binding energy of the exciton. ${ }^{36,37}$ These facts lead us to treat electronelectron interactions in long chains and arrive at an understanding of electronic states in luminescent polymers without loss of essential physics. We consider correlation by means of the extended Hubbard model. The remaining two summations of the Hamiltonian 1 are adopted to consider onsite- of $U$ strength-and nearest-neighbor site Coulomb repulsion-given by $V$. It is worthy to remember that, in the last term, the number operator $m_{n}$ is equal to $C_{n, \uparrow}^{\dagger} C_{n, \uparrow}+$ $C_{n, \downarrow}^{\dagger} C_{n, \downarrow}{ }^{38}$ Note that the Hubbard terms are properly expressed in such a way that the Hamiltonian remains Hermitian; i.e., the electron-hole symmetry is preserved.

Given the hybrid nature of the Hamiltonian, an equivalent semiclassical treatment should be applied. In other words, the classical equations of motions, expressed by the EulerLagrange equations

$$
\frac{\mathrm{d}}{\mathrm{d} t}\left(\frac{\partial\langle L\rangle}{\partial \dot{u}_{n}}\right)-\frac{\partial\langle L\rangle}{\partial u_{n}}=0
$$

must be simultaneously solved with the time-dependent Schrödinger equation for the $\pi$ electrons.

$$
\begin{aligned}
& \psi_{k, s}\left(n, t_{j+1}\right)=\sum_{l}\left[\sum_{m} \phi_{l, s}^{*}\left(m, t_{j}\right) \psi_{k, s}\left(m, t_{j}\right)\right] \\
& \times \mathrm{e}^{\left(-i \varepsilon_{l} \Delta t / \hbar\right)} \phi_{l, s}\left(n, t_{j}\right)
\end{aligned}
$$

in which $\Delta t$ is the time step chosen to carry out the numerical solution and $\left\{\phi_{l}(n)\right\}$ and $\left\{\varepsilon_{l}\right\}$ are the eigenstates and the eigenenergies, ${ }^{6,39}$ at the instant $t_{j}$ of the electronic Hamiltonian. ${ }^{14}$ 
The solution procedure is carried out self-consistently: by beginning with a given set of positions $\left\{y_{n}\right\}$, one solves the quantum mechanics equation thus obtaining a whole new set $\left\{y_{n}^{\prime}\right\}$. When the new set is sufficiently close to the previous one, the self-consistency is achieved.

It is important to note that, when solving the lattice part of the system through the Euler-Lagrange equations (2), a Newton-like force equation arises:

$$
\begin{aligned}
& F_{n}(t)=M \ddot{u}_{n}=-K\left[2 u_{n}(t)-u_{n+1}(t)-u_{n-1}(t)\right] \\
& \quad+\alpha\left[B_{n, n+1}-B_{n-1, n}+B_{n+1, n}-B_{n, n-1}\right]
\end{aligned}
$$

where the term $B_{n, n^{\prime}}=\Sigma_{k, s}{ }^{\prime} \psi_{k, s}^{*}(n, t) \psi_{k, s}\left(n^{\prime}, t\right)$ couples the electronic and lattice solutions. The form of this expression is important in order to consider thermal effects in the system in a simple and elegant way. Indeed, a slight modification of eq 4 that includes the sum of a white stochastic signal $\zeta(t)$ (in the sense that $\langle\zeta(t)\rangle \equiv 0$ and $\left.\left\langle\zeta(t) \zeta\left(t^{\prime}\right)\right\rangle=\Lambda \delta\left(t-t^{\prime}\right)\right)$ on its righthand side simulates random displacements of the carbon cores in the same way that temperature does. In order to keep the systems energy stable, a Stokes-like dissipation term is also added to eq 4 , which finally reads

$$
M \ddot{u}=-\gamma \dot{u}+\zeta(t)+F_{n}(t)
$$

where $F_{n}(t)$ stands for the $0 \mathrm{~K}$ situation. It should be noted that both the white signal $\zeta$ and the dissipation term $\gamma$ are connected to the systems temperature $T$ through the fluctuationdissipation theorem, $\Lambda=2 k_{\mathrm{B}} T \gamma M$. We have been using the approach of including temperature in organic semiconductors with great success by obtaining good qualitative experimental agreement. $^{40-47}$

\section{RESULTS AND DISCUSSION}

A systematic numerical investigation concerning the influence of temperature effects and an external electric field on the collisional process is performed in systems composed of 200site cis-polyacetylene chains with periodic boundary conditions. For the electric field, turned on quasi-adiabatically, ${ }^{42}$ the values used in the simulations varied from 0.5 to $2.0 \mathrm{mV} / \AA$ with an increment of $0.5 \mathrm{mV} / \AA$, whereas the temperature regimes considered ranged from 25 to $300 \mathrm{~K}$ with a step of $25 \mathrm{~K}$. The on-site electron-electron (e-e) interaction $(U)$ values considered here ranging from 0.5 to $1.0 \mathrm{eV}$, with an increment of $0.1 \mathrm{eV}$. The nearest-neighbor Coulomb repulsion strength is adopted as being $V=U / 3$.

In this paper, we report two different kinds of collisional process: one between an exciton and a polaron and another one between an exciton and a bipolaron. In order to better present our results, we divided each of the aforementioned kinds of reactive systems in separated subsections.

Collision between Polaron and Exciton. Quasi-particles in conducting polymers are usually formed by adding, removing, or even exciting electrons between the energy levels of the system. The changes imposed to the electrons reflect in lattice distortions and the rising of energy levels inside the gap. For the case discussed in this section, the positively charged polaron is represented by the solely occupied HOMO-1 level and the empty LUMO+1 level. On the other hand, the exciton is represented by electrons with an antiparallel spin configuration in the HOMO and LUMO levels, as shown in Figure 1

A first important result to be observed in our simulations is that the scattering dynamics is independent of the spin
CONDUCTION BAND

$$
\text { LUMO+1 }
$$

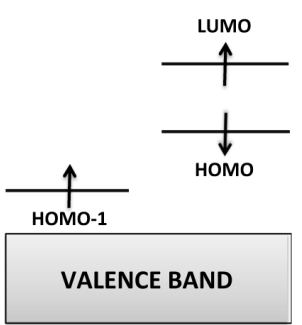

Figure 1. Schematic diagram of energy levels for a polymer chain containing a polaron and an exciton.

configuration presented by the system composed by a polaron and a exciton, considering on-site $\mathrm{e}-\mathrm{e}$ interactions smaller than $0.5 \mathrm{eV}$. In this way, in the absence of temperature, the polaron can pass through the exciton for on-site $\mathrm{e}-\mathrm{e}$ interaction strengths smaller than this value and considering an electric field strength of $2.0 \mathrm{mV} / \AA$. For regimes higher than this critical value, the polaron may be bounced back or be dissociated depending on the strength of the external electric field, as qualitatively described in ref 5 .

In this sense, Figure 2 presents the results concerning the collisional process between the polaron and the exciton for 100 $\mathrm{K}$. The time evolution of the system in terms of the staggered bond length order parameter defined as $\bar{y}(t)=(-1)^{n}\left[y_{n-1}(t)+\right.$ $\left.2 y_{n}(t)+y_{n+1}(t)\right] / 4$ and the charge density order parameter $\bar{\rho}(t)$ $=1-\left[\rho_{n-1}(t)+2 \rho_{n}(t)+\rho_{n+1}(t)\right] / 4$ is shown in Figure $2 \mathrm{a}$ and Figure $2 \mathrm{~b}$, respectively. These mean quantities are used in order to enable a better visualization of the dynamical process. Initially, the polaron is located at the 25 th site, whereas the exciton is positioned around the site 125. This separation guarantees that both structures initially act as independent particles. In the beginning of the simulation, the fast response of the polaron to the applied field of $2.0 \mathrm{mV} / \AA$ is clearly perceived. Before $25 \mathrm{fs}$, the charged structure is impelled by the electric field toward the exciton, whose position, in its turn, is only randomly affected by the lattice vibrations due to the fact that this structure presents no net charge. Before the collision, the polaron dynamics occurs as previously described in the literature. ${ }^{43}$ The main result obtained for this simulation concerns the product of the reactive mechanism between the polaron and the exciton. At approximately $375 \mathrm{fs}$ the structures collide generating a nearly complete disappearance of the polaron. This process gives rise to a final state where only the exciton remains in the lattice, although slightly modified due to the phonons arising from the collision.

One can confirm the aforementioned results by analyzing the charge density behavior, as shown in Figure $2 \mathrm{~b}$. The disappearing of the polaron after the collision is confirmed by the spreading of its charge over the chain at around $380 \mathrm{fs}$. Note that this time agrees with the vanishing of the lattice distortion signature of the structure presented in Figure 2a, therefore showing that the coupling between the lattice and charge is no longer present. The exciton, on the other hand, remains stable, as no oscillation of charge is observed for this neutral structure. This fact reaffirms the greater stability of the 

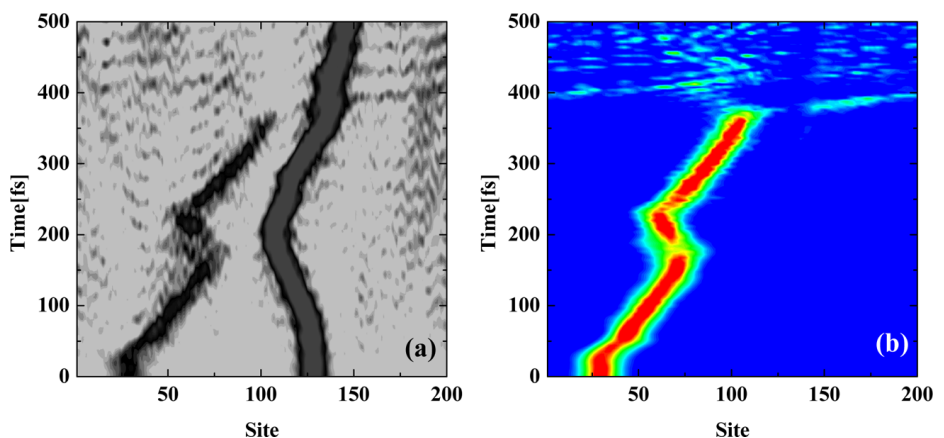

Figure 2. (a) Staggered bond length and (b) mean charge density time evolution for the polaron-exciton pair collisional process subjected to an electric field strength of $2.0 \mathrm{mV} / \AA$ and a temperature regime of $100 \mathrm{~K}$.
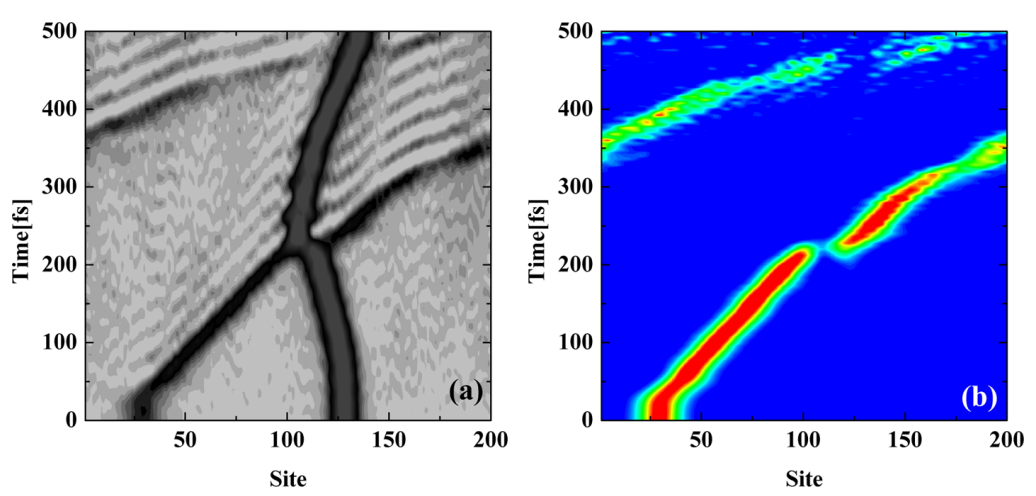

Figure 3. (a) Staggered bond length and (b) mean charge density time evolution for the polaron-exciton collisional process subjected to an electric field strength of $2.0 \mathrm{mV} / \AA$ and a temperature regime of $50 \mathrm{~K}$.

exciton compared to the polaron, a result that is widely reported in the literature. ${ }^{18}$

A last feature worthy to be mentioned for this set of simulations is that a similar pattern of disappearing polarons is observed for collisional processes carried out at higher temperatures and same electric field strength. For temperatures of $125,150,175,200,225,250,275$, and $300 \mathrm{~K}$, the collision between the exciton and the polaron resulted in a final state where only the exciton is present in the lattice. These results suggest that the dissociation of the polaron is, indeed, a phonon assisted process, which is an expected fact from the phenomenological point of view.

On the route of obtaining the phase transition temperature for the products of the collisional process between a polaronexciton pair, we consider $50 \mathrm{~K}$ for the thermal regime, maintaining all the remaining parameters unchanged. Figure $3 \mathrm{a}$ presents the lattice distortion profile of the polaron-exciton collision in this case. The first feature worthy to be mentioned is that, although the precollision process remains apparently unaffected from the qualitative point of view, the mean velocity of the polaron is considerably increased, as can be deduced from the slope of the particle's trajectory. This increase of velocity results in a sooner collision at approximately $225 \mathrm{fs}$, when compared with the $100 \mathrm{~K}$ situation. The fact that temperature negatively affects the charge carriers mobility is related to the fact that the competition between the systematic drift imposed by the electric field and the random vibration originated from thermal excitations is diminished in favor of the former. Therefore, the particle presents a smoother, and consequently faster, path through the lattice. The most strikingly important result, however, is related to the final product of the collisional process. One can clearly see, in Figure 3a, that two different well-defined localized lattice distortions arise from the collision. Each lattice defect can be associated with one of the initial structures, i.e., a polaron and an exciton. It can be seen that, in the present case, the polaron directly passes through the exciton, maintaining its integrity.

The mean charge density time evolution profile for the $50 \mathrm{~K}$ collisional system is presented in Figure $3 \mathrm{~b}$. Through this figure we can confirm that, in this lower temperature regime, the polaron remains stable even after the collision. One can see that the charge spreading reported in Figure $2 \mathrm{~b}$ is not present in Figure 3b. Moreover, the charge of the polaron remains strongly coupled to its lattice distortion, as can be inferred by the coincidence between the traits in Figures $3 \mathrm{a}$ and $3 \mathrm{~b}$. Thus, these results suggest that, for $2.0 \mathrm{mV} / \AA$, a thermal regime of 50 $\mathrm{K}$ conducts to a final state in which both the polaron and the exciton are stable structures. It is also important to note that, for this electric field strength, the same pattern is observed for smaller temperatures.

At this point, it should be emphasized that this so-called temperature phase transition on the final state is also electric field dependent. If one consider another electric field values, a shift in this critical temperature value is observed. Particularly, we observed that for electric fields of $0.5,1.0$, and $1.5 \mathrm{mV} / \AA$, temperatures higher than $150 \mathrm{~K}$ resulted in the dissociation of 
the polaron, similarly to that shown in Figure 2. On the other hand, temperatures smaller than $150 \mathrm{~K}$ induce a final state in which, similarly to Figure 3, both the polaron and the exciton are present.

In order to further investigate the formation of different products, we finish this subsection by performing an analysis of the occupation number. Figure 4 presents the time evolution of

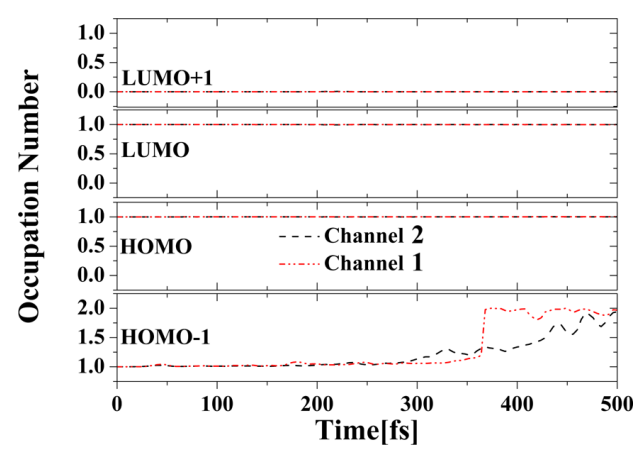

Figure 4. Time evolution of the occupation number of the intragap energy levels. The red lines represent the channel $1(100 \mathrm{~K})$, and the black lines represent the channel $2(50 \mathrm{~K})$.

the occupation number for the channel 1 , in which the collisional process at $100 \mathrm{~K}$ is considered, and for the channel 2, where the thermal regime is $50 \mathrm{~K}$. Note that whereas channel 1 (red lines) consists on the time evolution for the intragap levels related to Figure 2, channel 2 (black lines) is an analogous representative of Figure 3. Taking Figure 1 into consideration, one can clearly see that in the case of channel 1 the polaron dissociation is directly related to the returning of the energy levels to the valence and conduction bands. The behavior of the energy levels follows the pattern previously reported in our recent works. ${ }^{6,39}$

Collision between Bipolaron and Exciton. We now turn our attention to the collisional process between a positive bipolaron and an exciton. Figure 5 presents the schematic diagram of energy levels for a polymer chain containing these structures. Note that the bipolaron is represented by the absence of two electrons in HOMO-1 and LUMO+1 levels,

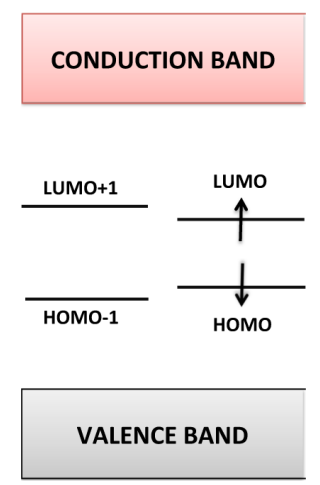

Figure 5. Schematic diagram of energy levels for a polymer chain containing an bipolaron and an exciton. thus yielding a $+2 e$ net charge. The exciton, on the other hand, is represented exactly as in the case of Figure 1.

Using the same parameters previously discussed, we now consider the bipolaron-exciton pair collision under effect of an electric field strength of $1.0 \mathrm{mV} / \AA$ and at a $100 \mathrm{~K}$ thermal regime. In this case, the bipolaron is initially positioned at the 125 th site, whereas the exciton is at site 25 . Figure 6a presents the time evolution for the lattice distortions. The lattice vibrations, imposed by the temperature effects, disturbs the system in such a level that the electric field is not able to drive a considerable velocity for the charge carrier. In this way, the bipolaron moves through the lattice performing a random walk dynamics imposed by the lattice fluctuations and the collision takes place at around $700 \mathrm{fs}$. After the structures begin to interact their wave functions interact strongly, giving rise to a mixed transition structure that is observed until about $850 \mathrm{fs}$. The recombination process is concluded with the formation of two different self-localized structures from this transition state. It can be clearly seen that the lattice distortion profile of the emerging structures appear to be different from the initial ones.

In order to unequivocally determine the nature of the products that arise from the collisional process, we resort to the time evolution profile of the charge density, as shown in Figure $6 \mathrm{~b}$. Indeed, we observe two distinct structures that follow exactly the path described by the lattice distortion. Also, by analyzing the charge density profile, one can conclude that each one of these structures are localized excitation that consists on the coupling of three charged quasi-particles: two originated from the bipolaron and one from the original exciton. Also, considering the distortion pattern, one concludes that the product of the bipolaron-exciton collision under this electric field and temperature regimes is a pair of trions, i.e., localized excitations consisting on the combination of three charged quasi-particles. The two new self-localized electronic states formed after the collision process can also be named "excited polarons". In fact, the excited polaron is equivalent to a charged trion, as can be inferred from their electronic states. In the electronic spectrum of a finite polymer chain containing a trion, there are two localized energy levels in the gap. This is analogous to the energy levels configuration for the exciton, as shown in Figure 1. For the negative trion, the lower level (HOMO) is occupied by one electron and the upper one (LUMO) by two; for the positive trion, the LUMO is occupied by one electron and the HOMO level is empty. Considering a positive trion, for example, when an electron located in the LUMO level moves to the HOMO, a photon can be emitted and the trion decays into a normal polaron. Furthermore, as a three-particle system, the trion possesses a larger binding energy and a deeper lattice distortion than the polaron does, as can be seen in Figure 6a.

The systematic set of simulations performed (but not presented for the sake of brevity) in this work allowed us to conclude that for electric fields between 0.5 and $1.5 \mathrm{mV} / \AA$ all temperature regimes yielded the same products from the bipolaron-exciton collisional process, i.e., a pair of trions. Therefore, in the search of different products, we considered the collision between the bipolaron and the exciton subjected to a electric field strength of $2.0 \mathrm{mV} / \AA$. Even at this regime, the same kind of products presented in Figure 6 are achieved for temperatures smaller than $100 \mathrm{~K}$. For this critical value, however, a whole different situation is observed as can be seen in Figure 7. In this case, the bipolaron is initially positioned at the 25 th site and the exciton at the 125th site. The higher 

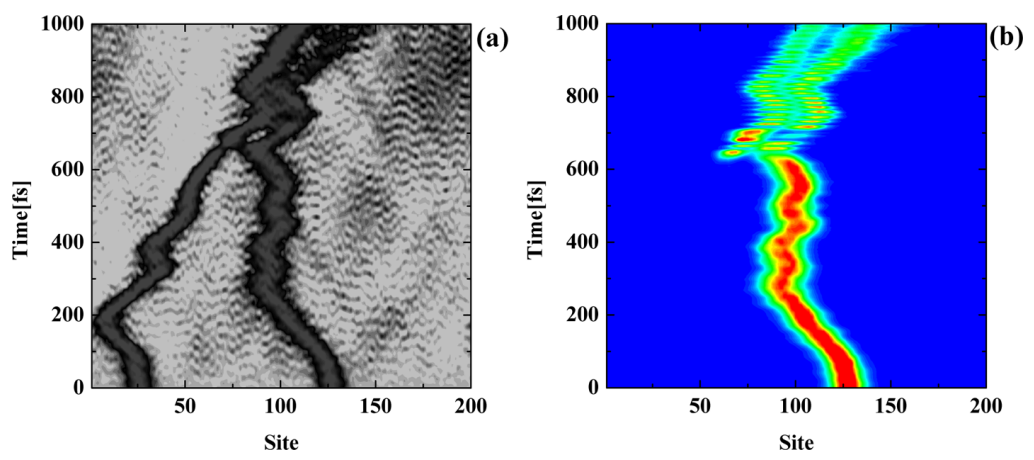

Figure 6. (a) Staggered bond length and (b) mean charge density time evolution for the bipolaron-exciton collisional process subjected to an electric field strength of $1.0 \mathrm{mV} / \mathrm{A}$ and a temperature regime of $100 \mathrm{~K}$.
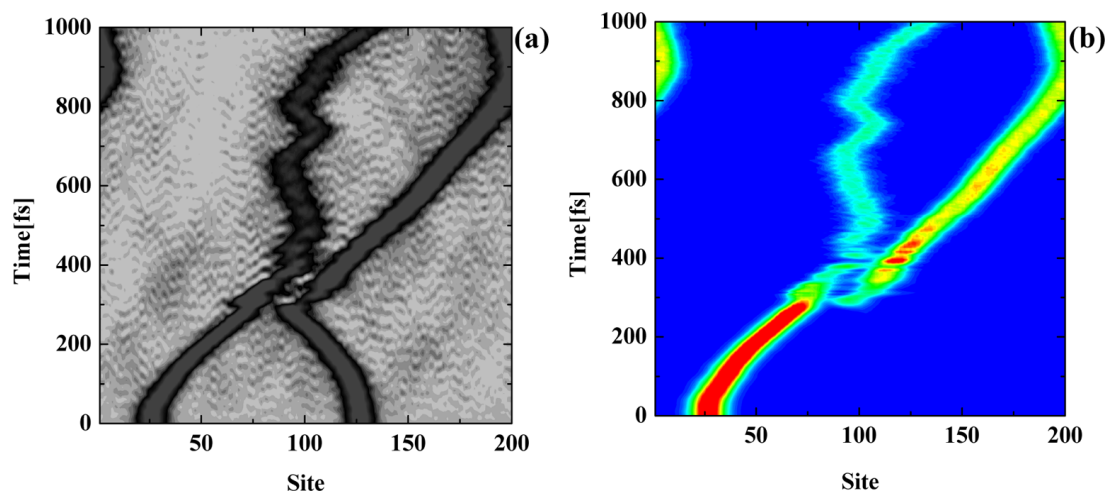

Figure 7. (a) Staggered bond length and (b) mean charge density time evolution for the bipolaron-exciton collisional process subjected to an electric field strength of $2.0 \mathrm{mV} / \AA$ and a temperature regime of $100 \mathrm{~K}$

electric field value induces a much sooner collision that takes place at around $300 \mathrm{fs}$.

Figure 7a shows that, after the collision, two self-localized lattice distortions with fairly different mobilities are present in the lattice. This is blatantly different from the results presented in Figure 6, in which the two arising trions perform an almost parallel path after the bipolaron-exciton annihilation. Also, the difference in the blurring of the figure related to each structure is another important indication of the difference in nature of each component of the reactive product. A more precise definition on the nature of the quasi-particles is given by the analysis of Figure $7 \mathrm{~b}$, which presents the mean charge density profile. Through an investigation of the typical responses to the applied electric field, the charge density values, and the dynamics pattern for the charge carriers in conjugated polymers, as reported in the refs 6 and 43, we can conclude that the more charged structure is a polaron, whereas the one with smaller field response is a polaron-exciton.

Figure 8 shows the occupation number time evolution for the case in which a polaron and a polaron-exciton are the final states after the recombination process. Besides the partial transfer between LUMO and LUMO+1 levels, it is observed a whole electron transference from HOMO to HOMO-1 level. These final states are the typical signature of the products observed for this regime. By observing first few 10 fs of Figure 8 very carefully, one can see that the occupation number of the LUMO level sharply decreases from 1 to 0 , while at the same

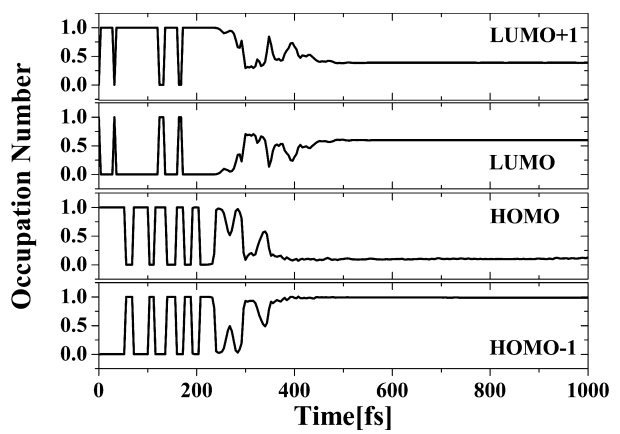

Figure 8. Time evolution of the occupation number of the intragap energy levels.

time the occupation number of LUMO+1 sharply increases from 0 to 1 . Furthermore, it is possible to note that this process occurs at other times during the first 200 fs. This change in occupation number reflects the fact that these two levels interchange positions. The exchange of the energy levels also occurs with the HOMO levels. However, after $400 \mathrm{fs}$, the energy levels exchange is no longer energetically favorable mainly due to the interplay between the electric field, temperature, electron-electron interactions, and the coupling between the bipolaron and the exciton when they begin to 
collide. A similar behavior has beeb recently reported in the literature. $^{44}$

The stability of the quasi-particles can be well described by the analysis of the energy levels time evolution. We finish our discussion by presenting, in Figure 9, the time evolution profile

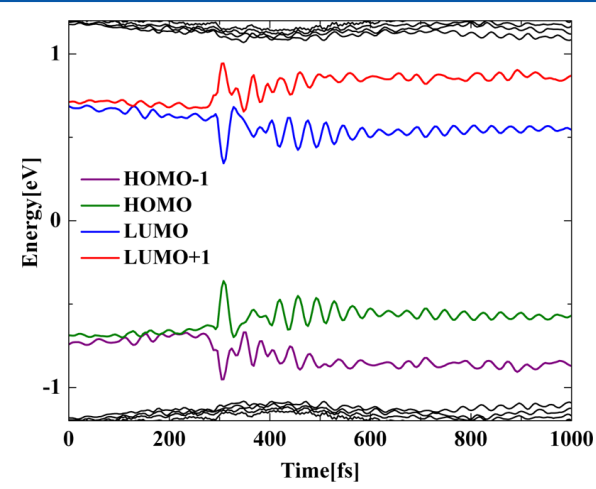

Figure 9. Time evolution of energy levels at the gap.

for the intragap energy levels for the cases shown in Figure 7. As can be seen from Figure 9, at the beginning there are four intragap levels, which are localized electron states caused by the presence of the bipolaron and the exciton in the lattice. According to their wave functions, we know that the purple and red lines correspond to the positive bipolaron levels HOMO-1 and LUMO+1, while the green and blue levels are related to the exciton HOMO and LUMO, respectively. Before the electric field is applied and in the absence of temperature effects, the two bipolaron intragap levels and the two exciton intragap levels are degenerate, which means that the ground state solution for a chain containing a bipolaron and an exciton is initially degenerated, as reported in ref 12 . However, considering the dynamical process, the degeneracy is removed by the first few femtoseconds of simulation due to temperature effects and the presence of an external electric field. Furthermore, when the bipolaron approaches the exciton, the interaction between them can also remove the degeneracy. Regarding the temperature influence on the degeneracy breaking, the equation for the hopping integral, showed in the previous section, takes into account the lattice displacements $\left(y_{n}\right)$ in order to calculate the hopping integrals between neighboring sites. These lattice displacements are randomically changed at each time step (dynamic disorder) when thermal effects are considered due to the action of thermal random forces, consequently causing random changes in the hopping integral values. Since the new eigenstates are obtained by diagonalizing the electronic part of the Hamiltonian at each step and this Hamiltonian matrix is exactly composed by the hopping integral values, the eigenstates will be different at each time step, which leads to the degeneracy breaking. Thus, the recombination process between the bipolaron and the exciton is noted to take place at approximately $300 \mathrm{fs}$, when the LUMO +1 and HOMO-1 levels move to the valence and conduction band, respectively, whereas the HOMO and LUMO+1 levels move toward the midgap region. Also, the resulting phonons produced from the recombination process are represented by the deeper oscillations of the energy levels inside the band gap. The final configuration of the energy levels shows a physical picture where both initial structure are substituted by a polaron and a polaron-exciton after the interaction between the original particles.

\section{SUMMARY AND CONCLUSIONS}

We have investigated the intrachain recombination process between two different charge carriers (polaron and bipolaron) and an exciton in conjugated polymers. The main goal was to find critical values of temperature that induced different products from the collisional process between the quasiparticles. It is shown that, unlike recently proposed at absolute zero temperature, in the case of polarons colliding with excitons, a spin-independent mechanism is obtained when temperature effects are included. This fact demonstrates the importance of considering such effects when describing the recombination process in a more realistic fashion. We performed a thorough description of the polaron exciton collision and determined the critical temperature for obtaining the integrity of the original polaron. We have also found this critical temperature value to be strongly field strength dependent. Regarding the intrachain recombination between a bipolaron and an exciton, two different regimes were also found. For low electric fields and temperatures, the products of the collision were two trions. Otherwise, a final state composed of a polaron and a polaron-exciton is observed.These results on obtaining critical values of controlled parameters for yielding different final states are crucial for mastering the technique of obtaining devices with the desired type and density of charge carriers. Therefore, this knowledge can be useful to improve the performance of organic based electronic devices such as polymer light-emitting diodes.

\section{AUTHOR INFORMATION}

\section{Corresponding Authors}

*E-mail wiliam@unb.br (W.F.d.C.).

*E-mail luiju@ifm.liu.se (L.A.R.J.).

\section{Notes}

The authors declare no competing financial interest.

\section{ACKNOWLEDGMENTS}

The authors gratefully acknowledge the Swedish Research Council (VR) for financial support, the Swedish National Infrastructure for Computing (SNIC), and the financial support from the Brazilian Research Councils CNPq, CAPES and FINATEC.

\section{REFERENCES}

(1) White, M. S.; et al. Ultrathin, Highly Flexible and Stretchable PLEDs. Nat. Photonics 2013, 7, 811-816.

(2) Lu, L. P.; Kabra, D.; Friend, R. H. Barium Hydroxide as an Interlayer Between Zinc Oxide and a Luminescent Conjugated Polymer for Light-Emitting Diodes. Adv. Funct. Mater. 2012, 22, 4165-4171.

(3) da Cunha, W. F.; Junior, L. A. R.; Gargano, R.; e Silva, G. M. Critical Temperature and Products of Intrachain Polaron Recombination in Conjugated Polymers. Phys. Chem. Chem. Phys. 2014, in press.

(4) An, Z.; Di, B.; Wu, C. Q. Inelastic Scattering of Oppositely Charged Polarons in Conjugated Polymers. Eur. Phys. J. B 2008, 63, 71-77.

(5) Sun, Z.; Liu, D.; Stafstöm, S.; An, Z. Scattering Process Between Polaron and Exciton in Conjugated Polymers. J. Chem. Phys. 2011, 134, 044906-5. 
(6) Ribeiro, L. A.; da Cunha, W. F.; Neto, P. H. O.; Gargano, R.; e Silva, G. M. Impurity Effects on Polaron-Exciton Formation in Conjugated Polymers. J. Chem. Phys. 2013, 139, 174903-6.

(7) Lu, Q.; Zhao, H.; Chen, Y.; Yan, Y. Dynamics Study of the Recombination of Polaron Pairs in Polymer Chain with Impurities. Physica B 2013, 412, 13-17.

(8) Lei, J.; Sun, Z.; Zhang, Y.; Xie, S. Effect of Spin Polarization on Exciton Formation in Conjugated Polymers. Org. Electron. 2011, 10, $1489-1494$.

(9) Di, B.; Meng, Y.; Wang, Y. D.; Liu, X. J.; An, Z. Electroluminescence Enhancement in Polymer Light-Emitting Diodes through Inelastic Scattering of Oppositely Charged Bipolarons. J. Phys. Chem. B 2011, 115, 9339-9344.

(10) Sun, Z.; Stafström, S. Spin-Dependent Polaron Recombination in Conjugated Polymers. J. Chem. Phys. 2012, 136, 244901-5.

(11) Sun, Z.; Li, Y.; Gao, K.; Liu, D. S.; An, Z.; Xie, S. J. Dynamical Study of Polaron-Bipolaron Scattering in Conjugated Polymers. Org. Electron. 2010, 11, 279-284.

(12) Sun, Z.; Li, Y.; Li, D. S.; An, Z.; Xie, S. Scattering Process Between Bipolaron and Exciton in Conjugated Polymers. Phys. Rev. B 2009, 134, 201310(R)

(13) Xie, Z.; Kang, Y. M.; An, Z.; Li, Y. C. Two-Dimensional Localized Vibrational Modes of Polythiophene Around a Bipolaron. Phys. Rev. B 2000, 61, 1096-1100.

(14) Lima, M. P.; e Silva, G. M. Dynamical Evolution of Polaron to Bipolaron in Conjugated Polymers. Phys. Rev. B 2006, 74, 224303-6. (15) Shimoi, Y.; Abe, S. Competition Between Polarons and Bipolarons in Nondegenerate Conjugated Polymers. Phys. Rev. B 1994, 50, 14781-14784.

(16) Furukawa, Y. Electronic Absorption and Vibrational Spectroscopies of Conjugated Conducting Polymers. J. Phys. Chem. 1996, 100, $15644-15653$.

(17) Genoud, F.; Guglielmi, M.; Nechtscheim, M.; Genesis, E.; Salmon, M. ESR Study of Electrochemical Doping in the Conducting Polymer Polypyrrole. Phys. Rev. Lett. 1985, 55, 118-121.

(18) Sun, Z.; Liu, D.; Stafström, S.; An, Z. Scattering Process Between Polaron and Exciton in Conjugated Polymers. J. Chem. Phys. 2011, 134, 044906-044911.

(19) Neto, P. H. O.; da Cunha, W. F.; Teixeira, J. F.; Gargano, R.; e Silva, G. M. Electron-Lattice Coupling in Armchair Graphene Nanoribbons. J. Phys. Chem. Lett. 2012, 3, 3039-3042.

(20) Ribeiro, L. A.; da Cunha, W. F.; Neto, P. H. O.; Gargano, R.; e Silva, G. M. Impurity Effects and Temperature Influence on the Exciton Dissociation Dynamics in Conjugated Polymers. Chem. Phys. Lett. 2013, 580, 108-114.

(21) Ribeiro, L. A.; da Cunha, W. F.; Neto, P. H. O.; e Silva, G. M. Dynamics of Photogenerated Polaron-Excitons in Organic Semiconductors. Phys. Proc. 2012, 28, 112-116.

(22) Johansson, A.; Stafström, S. Polaron Dynamics in a System of Coupled Conjugated Polymer Chains. Phys. Rev. Lett. 2001, 86, 36023605

(23) Johansson, A.; Stafström, S. Nonadiabatic Simulations of Polaron Dynamics. Phys. Rev. B 2004, 69, 235205-7.

(24) An, Z.; Wu, C. Q.; Sun, X. Dynamics of Photogenerated Polarons in Conjugated Polymers. Phys. Rev. Lett. 2004, 93, 216407-4.

(25) e Silva, G. M.; Terai, A. Dynamics of Solitons in Polyacetylene with Interchain Coupling. Phys. Rev. B 1993, 47, 12568-12577.

(26) Su, W. P.; Schrieffer, J. R.; Heeger, A. J. Solitons in Polyacetylene. Phys. Rev. Lett. 1979, 42, 1698-1701.

(27) Su, W. P.; Schrieffer, J. R.; Heeger, A. J. Solitons Excitations in Polyacetylene. Phys. Rev. B 1980, 22, 2099-2111.

(28) Pinheiro, C. S.; e Silva, G. M. Use of Polarons and Bipolarons in Logical Switches Based on Conjugated Polymers. Phys. Rev. B 2002, 65, 094304-5

(29) Stafström, S. Electron Localization and the Transition from Adiabatic to Nonadiabatic Charge Transport in Organic Conductors. Chem. Soc. Rev. 2010, 39, 2484-2499.
(30) Leng, J. M.; Jeglinski, S.; Wei, X.; Benner, R. E.; Vardeny, Z. V.; Guo, F.; Mazumdar, S. Optical Probes of Excited States in Poly(pPhenylenevinylene). Phys. Rev. Lett. 1994, 72, 156-159.

(31) Hsu, J. W. P.; Yan, M.; Jedju, T. M.; Rothberg, L. J.; Hsieh, B. R. Assignment of the Picosecond Photoinduced Absorption in Phenylene Vinylene Polymers. Phys. Rev. B 1994, 49, 712(R).

(32) Chandross, M.; Mazumdar, S.; Jeglinski, S.; Wei, X.; Vardeny, Z. V.; Kwock, E. W.; Miller, T. M. Excitons in Poly(para-Phenylenevinylene). Phys. Rev. B 1994, 50, 14702(R).

(33) Yu, Z. G.; Fu, R. T.; Wu, C. Q.; Sun, X.; Nasu, K. Excitons, Biexcitons, and the Band Gap in Poly(p-Phenylene Vinylene). Phys. Rev. B 1995, 52, 4849-4854.

(34) Guo, F.; Chandross, M.; Mazumdar, S. Stable Biexcitons in Conjugated Polymers. Phys. Rev. Lett. 1995, 74, 2086-2089.

(35) Soos, Z. G.; Ramasesha, S.; Galvao, D. S.; Etemad, S. Excitation and Relaxation Energies of Trans-Stilbene: Confined Singlet, Triplet, and Charged Bipolarons. Phys. Rev. B 1993, 47, 1742-1753.

(36) Ramasesha, S.; Pati, S. K.; Krishnamurthy, H. R.; Shuai, Z.; Brédas, J. L. Symmetrized Density-Matrix Renormalization-Group Method for Excited States of Hubbard Models. Phys. Rev. B 1996, 54, 7598-7600.

(37) Shuai, Z.; Brédas, J. L.; Pati, S. K.; Ramasesha, S. Exciton Binding Energy in the Strong Correlation Limit of Conjugated Chains. Phys. Rev. B 1998, 58, 15329-15331.

(38) Yu, Z. G.; Wu, M. W.; Rao, X. S.; Bishop, A. R. Excitons in Two Coupled Conjugated Polymer Chains. J. Phys.: Condens. Matter 1996, $8,8847-8857$

(39) Ribeiro, L. A.; da Cunha, W. F.; Neto, P. H. O.; Gargano, R.; e Silva, G. M. Dynamical Study of Impurity Effects on BipolaronBipolaron and Bipolaron-Polaron Scattering in Conjugated Polymers. J. Phys. Chem. B 2013, 117, 11801-11811.

(40) Ribeiro, L. A.; Neto, P. H. O.; da Cunha, W. F.; Roncaratti, L. F.; Gargano, R.; da Silva Filho, D. A.; e Silva, G. M. Exciton Dissociation and Charge Carrier Recombination Processes in Organic Semiconductors. J. Chem. Phys. 2011, 135, 224901-224906.

(41) Neto, P. H. O.; da Cunha, W. F.; Roncaratti, L. F.; Gargano, R.; e Silva, G. M. Thermal Effects on Photogeneration of Free Carriers in Organic Conductors. Chem. Phys. Lett. 2010, 493, 283-287.

(42) Ribeiro, L. A.; da Cunha, W. F.; Neto, P. H. O.; Gargano, R.; e Silva, G. M. Predicting the Equilibrium Structure of Organic Semiconductors with Genetic Algorithms. Chem. Phys. Lett. 2013, $555,168-172$.

(43) Ribeiro, L. A.; da Cunha, W. F.; Neto, P. H. O.; Gargano, R.; e Silva, G. M. Effects of Temperature and Electric Field Induced Phase Transitions on the Dynamics of Polarons and Bipolarons. New J. Chem. 2013, 37, 2829-2836.

(44) Sun, Z.; Stafstöm, S. Dynamics of Exciton Dissociation in Donor-Acceptor Polymer Heterojunctions. J. Chem. Phys. 2013, 138, 164905-8.

(45) Roncaratti, L. F.; Gargano, R.; de Oliveira Neto, P. H.; da Cunha, W. F.; da Silva Filho, D. A.; e Silva, G. M. TemperatureInduced Oscillating Electric Dipole in Conjugated Systems. Chem. Phys. Lett. 2012, 593, 214-217.

(46) Roncaratti, L. F.; Gargano, R; e Silva, G. M. Theoretical Temperature Dependence of the Charge-Carrier Mobility in Semiconducting Polymers. J. Phys. Chem. A 2009, 113, 14591-14594.

(47) Ribeiro Junior, L. A.; da Cunha, W. F.; e Silva, G. M. Exciton Recombination: Theoretical Insight into the Influence of High Density Regime of Excitons in Conjugated Polymers. J. Phys. Chem. B 2014, $118,5250-5257$. 


\title{
APPENDIX \\ PAPER XII
}

\section{Concentration Effects on Intrachain Polaron Recombination in Conjugated Polymers}

\author{
Luiz Antonio Ribeiro Junior \\ Wiliam Ferreira da Cunha \\ Antonio Luciano de Almeida Fonseca \\ Ricardo Gargano \\ Geraldo Magela e Silva
}

Phys. Chem. Chem. Phys., 2015, 17, 1299-1308 


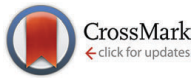

Cite this: Phys. Chem. Chem. Phys. 2015, 17, 1299

Received 6th October 2014, Accepted 18th November 2014

DOI: $10.1039 / c 4 c p 04514 a$

www.rsc.org/pccp

\section{Concentration effects on intrachain polaron recombination in conjugated polymers}

\author{
Luiz Antonio Ribeiro Junior, ${ }^{\text {a }}$ Wiliam Ferreira da Cunha, ${ }^{b}$ \\ Antonio Luciano de Almeida Fonseca, ${ }^{b}$ Ricardo Gargano ${ }^{c}$ and \\ Geraldo Magela e Silva ${ }^{\mathrm{b}}$
}

\section{Introduction}

Polymeric materials are now the focus of intensive research in order to expand the current knowledge about the science and technology of some optoelectronic devices, such as Polymer Light Emitting Diodes ${ }^{1}$ (PLEDs) and Organic Solar Cells ${ }^{2}$ (OSCs). By presenting unique traits, these materials are favorable to the development of cheaper and more efficient devices than their inorganic counterparts. In fact, for PLEDs the polaron recombination mechanism forming excited species is recognized as one of the most important physical processes to be fully understood in order to improve the electroluminescence yield in organic semiconductors. In this sense, relevant effort has been made to provide guidelines to the understanding of this mechanism in polymer based materials. ${ }^{3-12}$ Nonetheless, a detailed investigation about the impact of the polaronic concentration on the charge recombination process, albeit crucial, has not yet been performed and, therefore, requires further investigations.

Recently, Sun and Stafström used the Su-Schrieffer-Heeger (SSH) model to simulate the recombination process between an oppositely charged polaron pair in two coupled conjugated polymer chains. ${ }^{13}$ Their results show that the spin-dependent recombination mechanism can form an exciton or a bound interchain polaron pair depending on the interchain coupling

\footnotetext{
${ }^{a}$ Department of Physics, Chemistry and Biology (IFM), Linköping University, SE-58183 Linköping, Sweden. E-mail: luiju@ifm.liu.se

${ }^{b}$ Institute of Physics, University of Brasilia, 70.919-970, Brasilia, Brazil. E-mail: wiliam@fis.unb.br

${ }^{c}$ Department of Chemistry and Physics, University of Florida,

Quantum Theory Project, Gainesville, Florida, 32611, USA
}

and the external electric field strength. An et al. ${ }^{14}$ and Li et al. ${ }^{15}$ have also investigated the polaron recombination mechanism in the scope of a tight-binging approach. In general, their findings show that the external electric field can play an important role in the polaron-exciton formation through the scattering of two oppositely charged polarons. For field strengths smaller than $0.2 \mathrm{mV}^{-1}$, the two polarons recombine directly in a neutral excited state. Considering field strengths that lie in the range of $0.2-1.2 \mathrm{mV} \AA^{-1}$, the polarons scatter into a pair of two independent particles that are composed of a mixed state of polarons and excitons (polaron-excitons). Li and colleagues also briefly discuss the effect of electron-electron (e-e) interactions on the recombination dynamics between two oppositely charged polarons. They have reported that, for the products, a triplet exciton is much more energetically stable than a singlet one in the presence of on-site repulsions. Lei and collaborators have investigated the spin polarization effect on the formation of excited states via intrachain recombination of a polaron pair in conjugated polymers, using a version of the SSH model modified to include an external electric field and Coulomb interactions. ${ }^{16}$ Their results have pointed out that, neglecting e-e interactions, the yield of total excitons is independent of the spin orientation of the polaron pair. Moreover, the presence of e-e interactions shows a depression in the yield of singlet excited states in cases where the spin configuration between the polaron pair is not spin-parallel. As reported by Lei and collaborators, the e-e interaction strength does not change the content of the products formed from the recombination mechanism between two oppositely charged polarons, with spin-parallel or spin-antiparallel configuration, at all. Thus, in the present work, we can expect that relatively moderated variances in the e-e strength will not 
change the final products formed after the collision between the polarons. Very recently, our previous research studies have shown that impurity levels ${ }^{17}$ and temperature effects ${ }^{18}$ can change dramatically the recombination dynamics between two oppositely charged polarons. When influenced by impurities, the polaron-exciton formation also occurs for weak electric field strengths. The impurity levels can significantly improve the excitation yield in order to facilitate the polaron-exciton formation. Regarding the temperature influence on the recombination process, the results show that there are two possible channels in which (1) a neutral excitation is directly formed for temperatures below a critical regime and (2) a dimerized lattice is the final product of the collisional process for temperatures higher than the critical value.

From the results of all aforementioned research studies, one can see that further investigations of the polaron recombination mechanism, mainly considering the impact of the polaron concentration on the excited state formation, are strongly desired. We can see that the previous work in the literature usually deals with the interaction of a single pair of quasiparticles. As features such as electron-electron interactions as well as impurities showed a major influence over the final products of the system, one can readily conclude that the effect of different carrier concentrations is also a crucial issue, particularly when it is desired to study the behavior of the system in a more realistic physical situation.

In this manuscript, the impact of the charge carrier concentration on the intrachain recombination dynamics between oppositely charged polarons in conjugated polymers is numerically investigated considering the influence of an external electric field and Coulomb interactions. In the scope of a one-dimensional tight-binding approach, an Ehrenfest Molecular Dynamics is carried out in order to combine the Extended Hubbard Model and a modified version of the SSH model. The present investigation is aimed to provide a more realistic physical picture about the products formed after the recombination process by considering the concentration effects on the formation of excited states, which may provide guidance to improve the internal quantum efficiency in PLDEs.

\section{Methodology}

The model Hamiltonian is given by $H=H_{\mathrm{SSH}}+H_{\mathrm{ee}}$. The first part in this equation is the SSH-type Hamiltonian ${ }^{19,20}$ modified to include an external electric field and the Brazovskii-Kirova symmetry-breaking, in order to simulate a conjugated polymer chain with periodic boundary conditions. The SSH hamiltonian assumes the form

$$
H_{\mathrm{SSH}}=-\sum_{n, s}\left(t_{n, n+1} C_{n+1, s}^{\dagger} C_{n, s}+\text { h.c. }\right)+\sum_{n} \frac{K}{2} y_{n}{ }^{2}+\sum_{n} \frac{p_{n}{ }^{2}}{2 M},
$$

where $n$ labels the chain sites. Here, $y_{n} \equiv u_{n+1}-u_{n}$ in which $u_{n}$ is the lattice displacement of a particular site. $p_{n}$ is the conjugated momentum to $u_{n}, K$ is the harmonic constant for a $\sigma$ bond and $M$ is the mass of a $\mathrm{CH}$ group. The operator $C_{n, s}^{\dagger}$ $\left(C_{n, s}\right)$ creates (annihilates) a $\pi$-electron with spin $s$ at the $n$th site. $t_{n, n+1}=\mathrm{e}^{-i \gamma A(t)}\left[\left(1+(-1)^{n} \delta_{0}\right) t_{0}-\alpha y_{n}\right]$ is the transfer integral, where $t_{0}$ is the transfer integral of a $\pi$-electron between neighboring sites in the undimerized chain, $\alpha$ is the electron-phonon coupling, and $\delta_{0}$ is the Brazovskii-Kirova symmetry-breaking term. ${ }^{21} \gamma \equiv e a /(\hbar c)$, with $a$ being the lattice parameter, $e$ the absolute value of the electronic charge, and $c$ is the speed of light. The external electric field is introduced in the model through the time-dependent vector potential, in which $\mathbf{E}=$ $-(1 / c) \dot{\mathbf{A}}^{22}$ The last term in the model Hamiltonian denotes the contribution of the Coulomb interactions and can be written as

$$
\begin{aligned}
H_{\mathrm{ee}}= & U \sum_{i}\left(C_{i, \uparrow}^{\dagger} C_{i, \uparrow}-\frac{1}{2}\right)\left(C_{i, \downarrow}^{\dagger} C_{i, \downarrow}-\frac{1}{2}\right) \\
& +V \sum_{i}\left(n_{i}-1\right)\left(n_{i+1}-1\right),
\end{aligned}
$$

where $n_{i}=C_{i, \uparrow}^{\dagger} C_{i, \uparrow}+C_{n, \downarrow}^{\dagger} C_{i, \downarrow}$, and $U$ and $V$ are the on-site and nearest-neighbor electron-electron repulsion strengths, respectively. ${ }^{23}$ The parameters used here are recognized as standard and have the following values: $t_{0}=2.5 \mathrm{eV}, M=1349.14 \mathrm{eV} \times$ $\mathrm{fs}^{2} \AA^{-2}, K=21 \mathrm{eV} \AA^{-2}, \delta_{0}=0.05, \alpha=4.1 \mathrm{eV} \AA^{-1}$, and $a=1.22 \AA$. These values have been used successfully in previous simulations. ${ }^{24-30}$

The lattice backbone dynamics is described in a classical approach by a Newtonian equation $M \ddot{u}_{n}=F_{n}(t)$. Where,

$$
\begin{aligned}
F_{n}(t)= & M \ddot{u}_{n}(t)=-K\left[2 u_{n}(t)-u_{n+1}(t)-u_{n-1}(t)\right] \\
& +\alpha\left[B_{n, n+1}(t)-B_{n-1, n}(t)+B_{n+1, n}(t)-B_{n, n-1}(t)\right] .
\end{aligned}
$$

$F_{n}(t)$ represents the force experienced by a particular site $n$. Here, $B_{n, n^{\prime}}(t)=\sum_{k, s}^{\prime} \psi_{k, s}^{*}(n, t) \psi_{k, s}\left(n^{\prime}, t\right)$ is the term that couples the electronic and lattice parts of the model Hamiltonian. ${ }^{31}$ The primed summation represents a sum over occupied states. Eqn (3) can be numerically integrated using the method

$$
\begin{gathered}
u_{n}\left(t_{j+1}\right)=u_{n}\left(t_{j}\right)+\dot{u}_{n}\left(t_{j}\right) \Delta t, \\
\dot{u}_{n}\left(t_{j+1}\right)=\dot{u}_{n}\left(t_{j}\right)+\frac{F_{n}\left(t_{j}\right)}{M} \Delta t .
\end{gathered}
$$

In its turn, the time evolution of the electronic part is performed by solving the time-dependent Schrödinger equation (TDSE). The wave functions are constructed by means of a linear combination of instantaneous eigenstates of the electronic Hamiltonian. In this way, the solutions of the TDSE can be put in the form

$$
\psi_{k, s}\left(n, t_{j+1}\right)=\sum_{l}\left[\sum_{m} \phi_{l, s}^{*}\left(m, t_{j}\right) \psi_{k, s}\left(m, t_{j}\right)\right] \times \mathrm{e}^{\left(-i \varepsilon_{l} \Delta t / \hbar\right)} \phi_{l, s}\left(n, t_{j}\right),
$$

where $\left\{\phi_{l}(n)\right\}$ and $\left\{\varepsilon_{l}\right\}$ are the eigenfunctions and the eigenvalues of the electronic part for the Hamiltonian at a given time $t_{j}$, respectively. ${ }^{32}$ At time $t_{j}$ the wave functions $\left\{\psi_{k, s}\left(n, t_{j}\right)\right\}$ are expressed as an expansion of the eigenfunctions $\left\{\phi_{l, s}\right\}$ : $\psi_{k, s}\left(n, t_{j}\right)=\sum_{l=1}^{N} C_{l, k}^{s} \phi_{l, s}(n)$, in which $C_{l, k}^{s}$ are the expansion coefficients. Thus, the occupation number for each eigenstate $\phi_{l, s}$ is 
$\eta_{l, s}\left(t_{j}\right)=\sum_{k}^{\prime}\left|C_{l, k}^{s}\left(t_{j}\right)\right|^{2}$, which provides useful information about the redistribution of electrons among the energy levels.

\section{Results and discussion}

The main focus of the present work is to investigate the combined effects of different charge carrier concentrations and electric field values over the products of polaron scattering. In order to do so, we make use of polymer chain lengths of 200, $400,600,800$, and 1000 sites, depending on the number of charge carriers initially present. Accordingly, we considered 2, $4,6,8$, and 10 polarons as the initial state of the system and investigated the dynamics of these quasi-particles subjected to electric field strengths ranging from 0.1 to $2.0 \mathrm{mV} \AA^{-1}$ with an increment of $0.1 \mathrm{mV} \AA^{-1}$. In order to better consider interaction between the quasi-particles we included electron-electron correlation through the extended Hubbard formalism with $U=1.0 \mathrm{eV}$ and $V=U / 3$, in accordance with values recently used in the literature. ${ }^{33-39}$

We begin our discussion by presenting schematic diagrams of the energy levels for the different initial configurations considered in our work. In Fig. 1, " $\mathrm{H}$ " stands for the Highest Occupied Molecular Orbital (HOMO) level, " $\mathrm{H}-1$ " is the level immediately below it and so on. Analogously, "L" represents the Lowest Unoccupied Molecular Orbital (LUMO) level, "L + 1" the next one and so forth. Fig. 1(a) to (e) show how 2, 4, 6, 8, and 10 polarons were created in each particular chain by placing the electrons (represented by arrows) in the corresponding levels. The dynamics is carried out through a time evolution starting from the initial state described by Fig. 1. In all the cases we simulated initial carriers with opposite charges, so that collisions would take place when the electric field is turned on. From the experimental point of view, it is known that the generation dynamics of polarons from relaxed excited states in conjugated polymers may occur through two different channels: (1) impurity induced in the absence of an external electric field and (2) electric field induced in a pristine lattice, where the photocarriers are charged polaron pairs created directly. ${ }^{40}$ Moreover, it is well accepted that only a small part of the excited states generate free polarons in the absence of the electric field. On the other hand, when the influence of such an effect is taken into account, a substantial amount of excitons may relax into polaronic states forming stable charge carriers, which are useful to generate electrical current. Thus, the charge carriers result from the field-induced dissociation of excitons, which are primarily formed by photoexcitations. In our simulations, the initial configuration for the charge carrier mimics a photoexcitation mechanism followed by exciton relaxation, in which a substantial ratio of the excited states is converted into polaronic states. In polyacetylene lattices, at a half-filling band, the electronic band gap is approximately $1.8 \mathrm{eV}$. It was shown that the most probable dipole-allowed transitions are those from $\varepsilon_{i}^{\mathrm{v}}$ to $\varepsilon_{i}^{\mathrm{c}}(i=1,2,3)$, where $\varepsilon_{i}^{\mathrm{v}}$ and $\varepsilon_{i}^{\mathrm{c}}$ are the $i$ th energy level in valence (from the top) and conduction (from the bottom), respectively. The transition dipole moments between $\varepsilon_{i}^{\mathrm{V}}$ and $\varepsilon_{i}^{\mathrm{c}}$ considering $i$ raging from 1 to 4, for example, are 11.6, 11.1, 10.4, and $9.5 \mathrm{e} \AA$, and their transition energies are 1.76, 1.78, 1.81 , and $1.85 \mathrm{eV}$, respectively. ${ }^{29}$ Therefore, the numerical procedure adopted here, to generate the initial configuration, can represent fairly well the photoexcitation mechanics and (a) - 2 Polarons

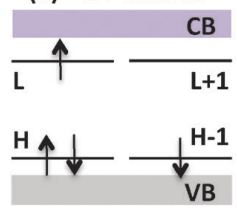

(b) -4 Polarons

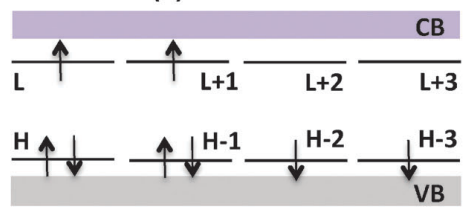

(c) -6 Polarons

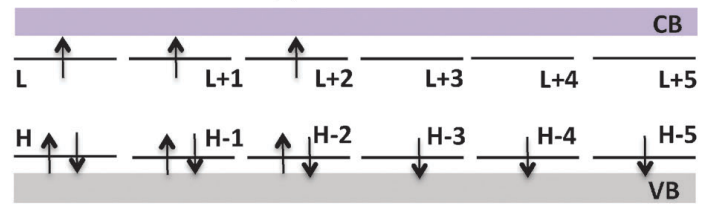

\section{(d) -8 Polarons}

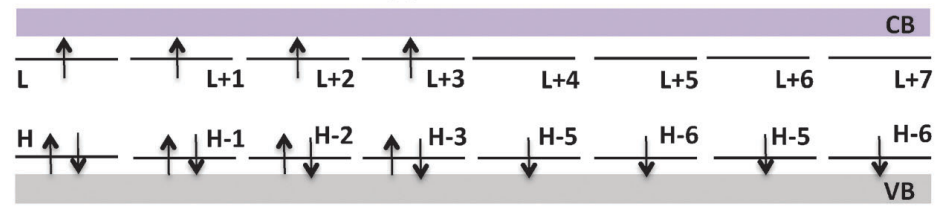

(e) -10 Polarons

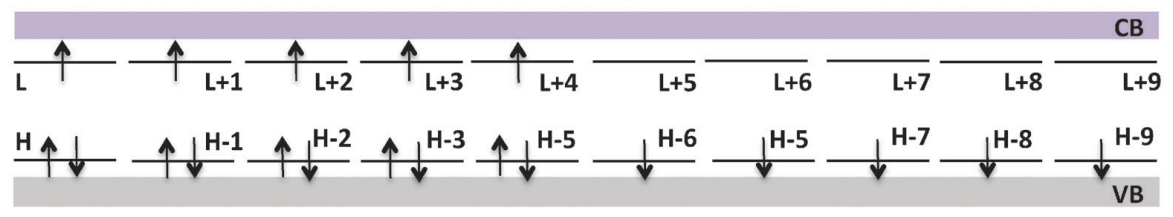

Fig. 1 Schematic representation of the energy levels for systems with different densities: (a) 2 polarons, (b) 4 polarons, (c) 6 polarons, (d) 8 polarons, and (e) 10 polarons. "CB" and "VB" stand for the conduction and the valence band, respectively. 
Paper
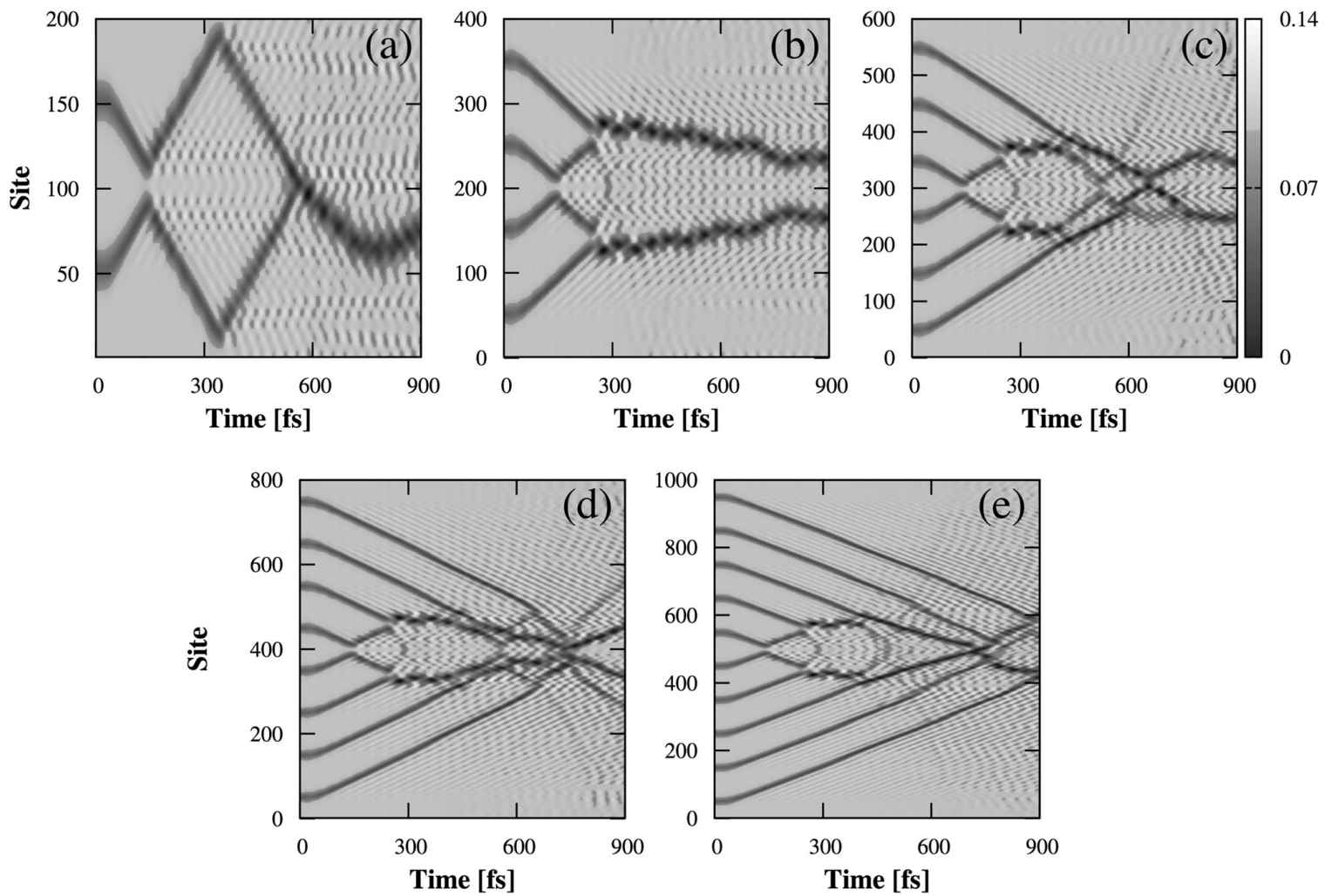

Fig. 2 Staggered bond-length time evolution for an electric field strength of $0.5 \mathrm{mV} \AA^{-1}$ for all the initial states in Fig. 1.

the subsequent exciton relaxation in order to generate free polarons.

Before we discuss the actual product nature of the polaron scattering, it is interesting to perform a general qualitative discussion on the influence of the carrier concentration on the product formation mechanism. In order to do so, we investigate the $0.5 \mathrm{mV} \AA^{-1}$ electric field induced dynamics of systems composed of the initial states presented in Fig. 1. Fig. 2 shows the staggered bond-length time evolution for this field strength, considering all different concentrations from 2 polarons in part (a) up to 10 polarons in part (e). The first feature the lattice distortion patterns suggest is that the product formed after the collisional process is strictly dependent on the initial polaron concentration. This can be inferred from the different shapes and patterns of the structures that arise from the scattering of the initial quasi-particles. As it will be further discussed, these systems can present very different products even when the same initial carrier concentration is considered. Moreover, as the charge carrier density $\left(N_{\mathrm{QP}} / n\right)$, in which $N_{\mathrm{QP}}$ is the number of quasi-particles and $n$ is the number of sites, is exactly the same, one can conclude that the products formed after the collisional process are concentration dependent instead of density dependent. In this work, the term "concentration" stands for a measure of the number of structures present in each chain, regardless of its length.
It is well known that two acoustic polarons with the same charge and antiparallel spins can recombine to form an acoustic bipolaron. These structures are spinless charge carriers and possess charge $\pm 2 e$. Bipolarons can be created in organicbased materials when the charge injection or the photoexcitation mechanism results in a large concentration of polarons. Recently, Di et al. have investigated the combination and scattering of two polarons with same charges and or antiparallel spin in conjugated polymers in the framework of a tight-binding approach. ${ }^{7}$ Their results have shown that collisions between polarons with the same charge and parallel spin are essentially elastic due to strong Pauli repulsion, whereas the combination between the polarons with the same charge and antiparallel spin generates a singlet bipolaronic state. To enable the bipolaron formation, the charge carriers with the same sign should accumulate in a certain region of the lattice. Thus, in the studies performed by Di et al., a conjugated polymer lattice with fixed ends was used in order that the polarons were accumulated at one of the extremities of the polymer. Note that, in our simulations, due to the periodic boundary conditions and concentration of polarons with opposite charge, the accumulation of polarons with the same charge in a certain region of the lattice is not favored. Moreover, the polarons with the same charge signal have spin parallel configuration (Fig. 1), which avoids the recombination between them to form new charged species. 

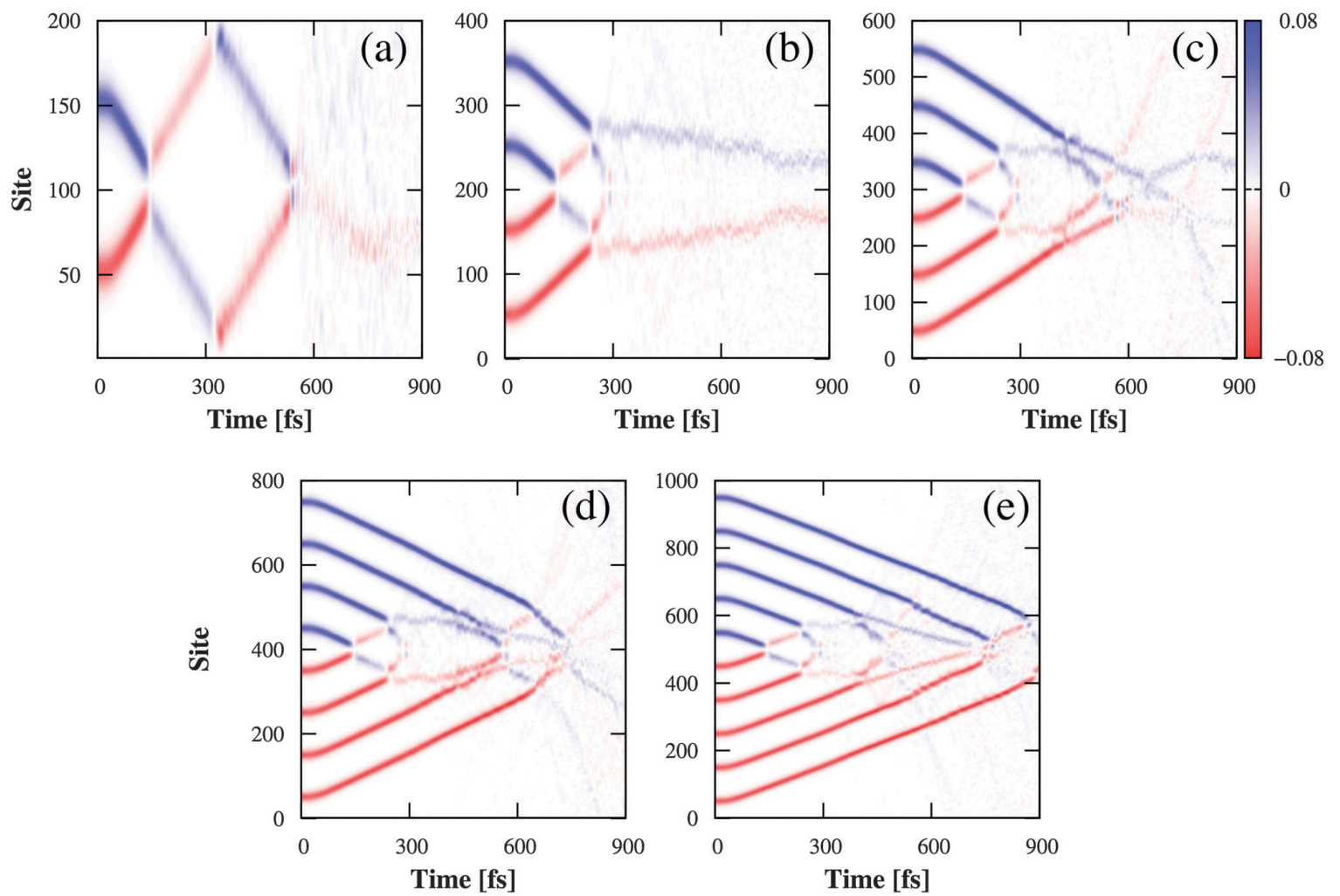

Fig. 3 Mean charge density time evolution for an electric field strength of $0.5 \mathrm{mV} \AA^{-1}$ for the cases shown in Fig. 2.

Therefore, we cannot expect the formation of bipolarons in the present work.

Fig. 3 depicts the time evolution of the mean charge density related to the cases presented in Fig. 2, where the figure labels are in connection with the cases just presented. By analyzing the mean charge density profile one can strictly assess the nature of the products. In Fig. 3(a), in which two polarons are initially considered, we observe a pair of polaron-excitons arising after the first collision, but a quasi-neutral excited state is also observed as the final state after the second collision. In other words, this quasi-neutral state is the final product of the collision between oppositely charged polaron-excitons, which work as complex products. Fig. 3(b) shows the collision between four polarons, giving rise to neutral excitations and also to polaron-excitons. Note that the first collision between the two inner polarons gives rise to a pair of polaron-excitons that subsequently collide with the two remainder outer polarons. After that, the collision between the polarons and the formed polaron-excitons forms two neutral excited states. This means that a recombination process between a charged state and a charged excited state tends to form only one neutral excited state. Interestingly, for the higher concentration regimes, Fig. 3(d) and (e), mixed final states between polaron-excitons and neutral excitations are achieved. For higher concentrations, one can see that it is difficult to distinguish between the nature of the products, but a general pattern is to observe that collisions at higher concentrations tend to favor the formation of polaronexcitons over neutral excitations. This pattern is confirmed by observing that, whereas in Fig. 3(a) the presence of neutral excitations is dominant, when the higher concentration cases of 3(d) and (e) are considered, one observes a higher ratio of polaron-excitons to neutral excitons. It should also be noted that the high degree of symmetry observed for small concentrations is lost for systems with a higher number of initial carriers, as shown in Fig. 3(c) to (e). This is mainly due to the higher amount of phonon states that is observed in these situations, and it is in close relation to the observed polarons.

It is worth mentioning here that the dissipative dynamics of polarons in conjugated polymers can be considered by including a damping term, a feature missing in the present work. Furthermore, the electric field is adiabatically turned on, reaching its full strength at about $75 \mathrm{fs}$, and is not turned off in these simulations. Thus, the polaron cannot lose its energy, at least before the collision process. Recently, the polaron dissociation in an organic molecule was theoretically investigated in the presence of dissipation. ${ }^{41}$ It was shown that the polaron's velocity is inversely proportional to the damping, which means that the relation between mobility and temperature is a power law. The damping term can suppress the step-like behavior of the polaron's velocity with regard to electric fields. Moreover, it 


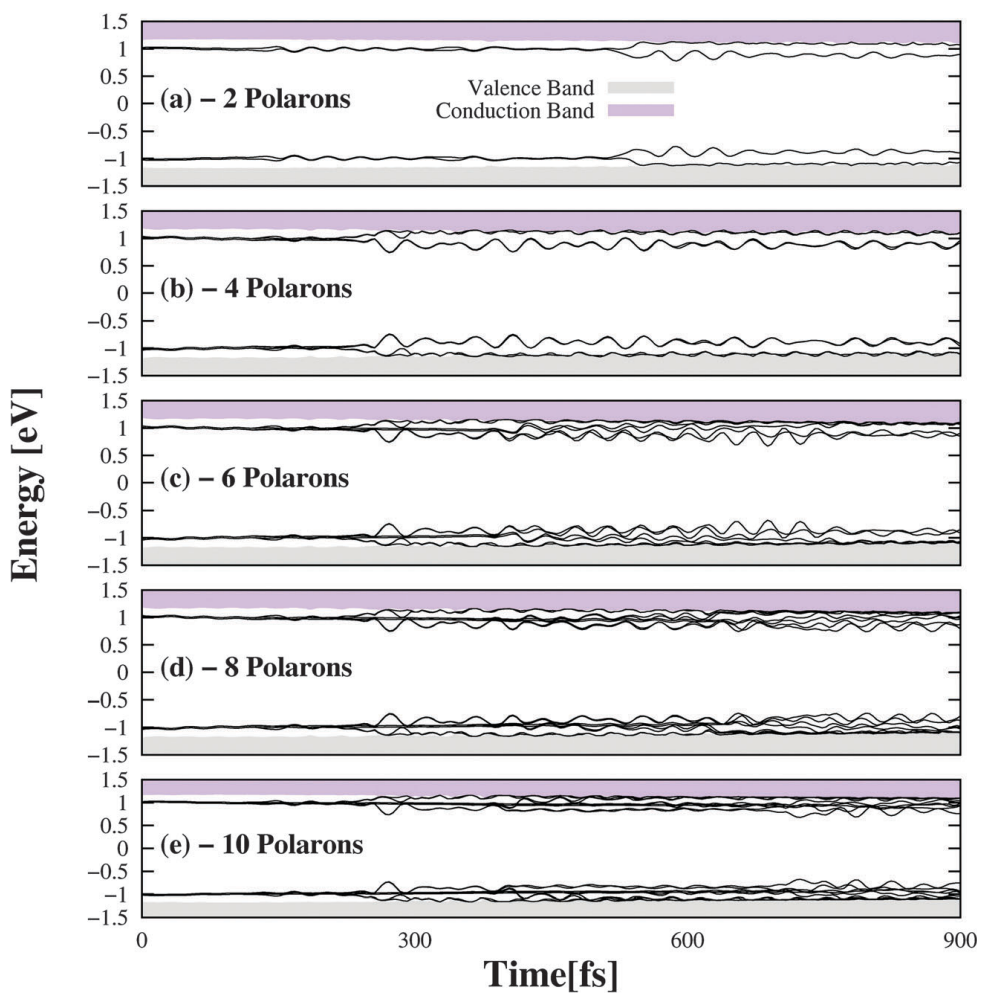

Fig. 4 Energy level time evolution for the systems shown in Fig. 2.

was obtained that, in the presence of a dissipative term, the threshold field that dissociates a polaron is reduced. As the main goal in this work is to figure out the possible products formed after the recombination mechanism between several oppositely charged polarons, we should ensure that these charge carriers might keep its integrity until the moment of the collision. So, the polaron dissipation mechanism is avoided in this work.

Another interesting manner to investigate the nature of the final states of the collisional process, as well as its evolution, is the study of its energy levels. It is well known that neutral excitations are more stable structures than the charged excitations. As a consequence, the energy levels related to the neutral excitations are positioned deeply inside the band gap, whereas the levels associated with the charged carriers and charged excitations lie in the band gap in regions closer to the valence and conduction bands. ${ }^{13,17}$ In this way, Fig. 4 confirms the previous discussion on the concentration dependence of the final products of the collision. The several levels inside the gap at the beginning of the simulation are a typical fingerprint of the presence of polarons. In this figure, the difference in creation time of the final structures is quite clear. Also, the presence of the neutral excitations can be easily noted in Fig. 4(a) and both the neutral excitation and the polaronexciton in Fig. 4(b). For the other concentrations, the mixture between neutral excitation and polaron-exciton states is strongly presented by the entanglement of the energy levels in the final part of the simulation. However, the dominant presence of states near the conduction and valence band inside the energy gap is an indication that, for systems of higher densities, the polaron-exciton formation is stimulated.

After discussing the results of different products depending on the concentration of charge carriers, we now turn our attention to a particular collision between three negative and three positive polarons subjected to an electric field strength of $0.6 \mathrm{mV} \AA^{-1}$ in a 600 sites polymer chain. Fig. 5(a) presents the bond-length and Fig. 5 (b) depicts the mean charge density time evolution of the system. Apart from the higher electric field
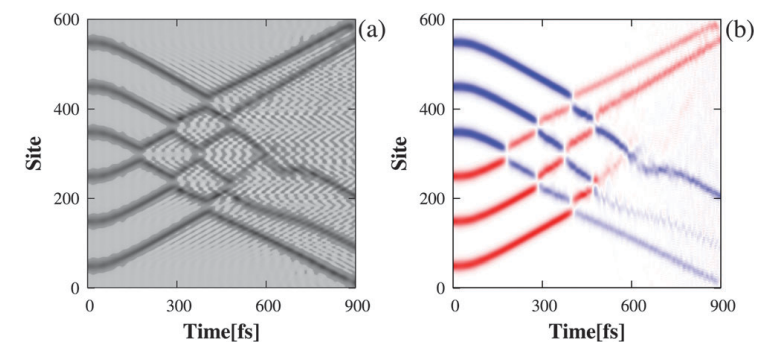

Fig. 5 (a) Staggered bond-length and (b) mean charge density time evolution for an electric field strength of $0.6 \mathrm{mV} \AA^{-1}$ of a polymer chain in the presence of three polaron-pairs. 

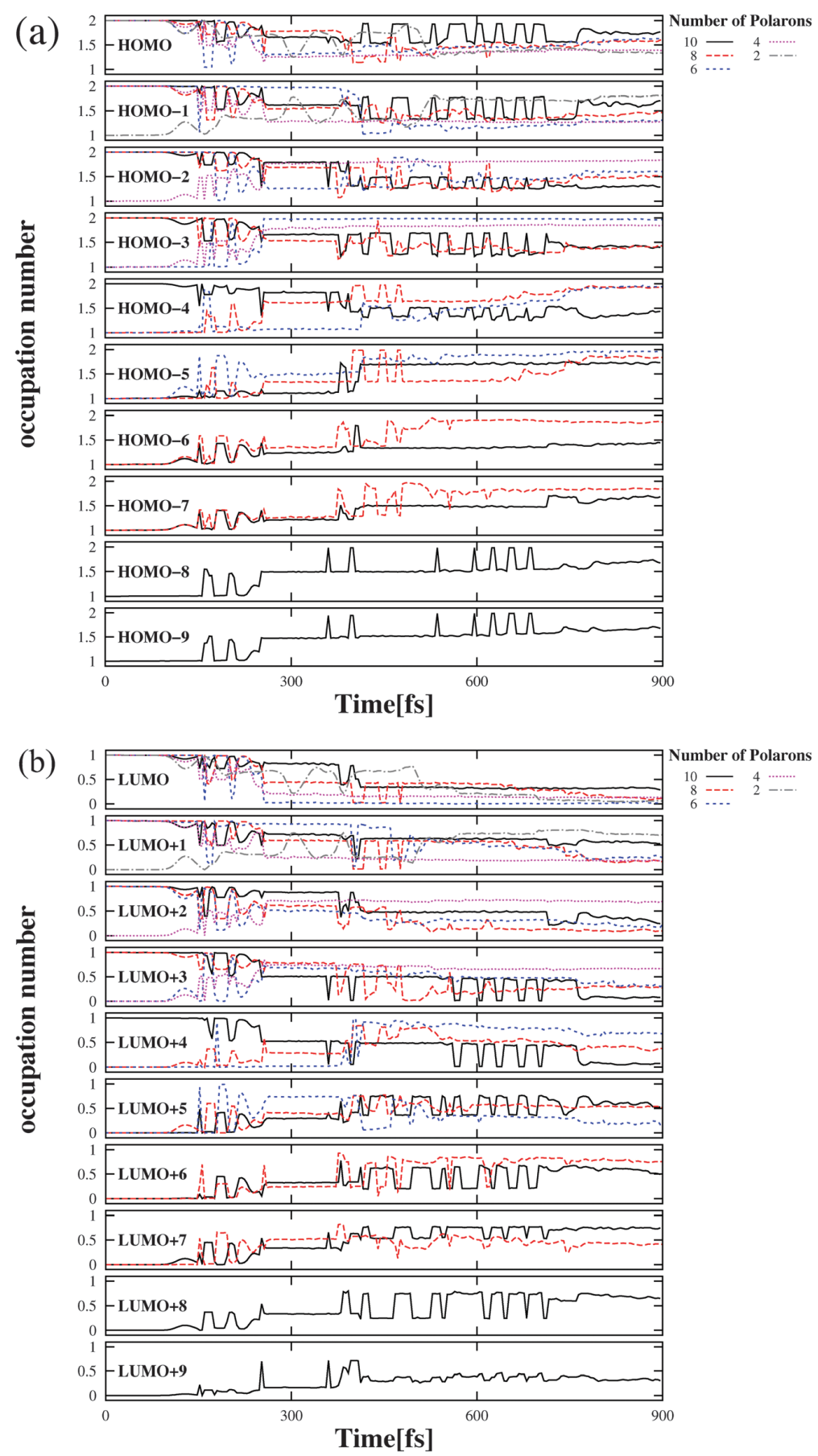

Fig. 6 Time evolution of (a) HOMO and (b) LUMO levels of the initial states presented in Fig. 2

applied, this situation is analogous to the simulation presented in Fig. 2(c) and, nevertheless, one can see that the formed products are quite different. After several mutual collisions, the final state is composed of polaron-exciton and neutral excitation states. This behavior is completely different to the results presented in Fig. 3(c), in which the final state is dominated by neutral excitations. Therefore, one should conclude that, similar to higher concentrations, higher electric 
field values also play the role of shifting the equilibrium towards the formation of more polaron-excitons rather than excitons.

Fig. 6 presents the time evolution of the (a) HOMO and (b) LUMO levels relative to the systems presented in Fig. 2. A quick glimpse to the figures allows one to observe the complementarity of the occupations between HOMO and LUMO levels. In other words, each HOMO level gains whatever fraction of electrons lost by the corresponding LUMO and vice versa. After around 150 fs of simulation, Fig. 6(a) shows that the electron begins to leave HOMO towards other energy levels. A similar pattern is observed for the LUMO in Fig. 6(b). As a general rule, it can be said that the simulations with higher concentrations present the lowest occupation of the LUMO and the highest occupation of the HOMO. This is directly connected to the higher concentration of polaron-exciton that results from these collisional processes. As a practical example, if one follows the line corresponding to the initial 10 polarons case, it is clear that although the LUMO presents a relative small occupation, it is emptied in a slower pace when compared to cases with smaller concentrations. This happens because the large number of carriers causes a rather complex succession of collisions that yields a final stable state at a longer time. It is worth mentioning here some important aspects regarding the final electronic occupation for the energy levels and the energy of the phonons after the collision process. In the relevant work performed by Sun and Stafström, ${ }^{42}$ it was shown that the charge distribution in higher levels inside the conduction band is mostly due to the energy excess of the electron, provided by the energy level offset, in the exciton dissociation mechanism developed by them, i.e., the charge may occupy the LUMO +8 even in the absence of an external electric field. Note that, for the results reported in the present manuscript, there is no exciton dissociation mechanism, instead, there is a recombination mechanism between oppositely charge carriers, which may contribute to the formation of excited states. Considering now the energy released in the collision process and the subsequent generation of phonons, studies performed by An et al. shown that, for electric field strengths higher than $1.2 \mathrm{mV}^{-1}$, the two oppositely charged polarons will break into irregular lattice vibrations after their collision. At the same time, the electron-hole localized in the lattice defects become free charges, where the electron and the hole move for different sides of the lattice driven by the electric field. In this case, no neutral excitation is formed (as can be inferred from the results presented in Fig. 5).

We finish our discussion by presenting the most important results of this work in Fig. 7, which is an extension of the analysis performed in the previous figures for other values of electric field strengths. Fig. 7(a) presents the sum of all the LUMO levels in the gap as a function of the applied electric field for all the simulated concentrations. Fig. $7(\mathrm{~b})$ is the equivalent figure for the HOMO. Thus, Fig. 7 consists of a useful tool to summarize our results besides providing new important results concerning the electric field dependence of the collision products. The first interesting feature of Fig. 7 regards the definition of a critical electric field value of $1.0 \mathrm{mV} \AA^{-1}$, above which, for all the simulated systems, a pristine lattice is obtained as the final state of the system. One can see that, for all values of electric fields greater than $1.0 \mathrm{mV} \AA^{-1}$, all the LUMO levels are back to the HOMO position, thus resulting in a lattice free of defects.
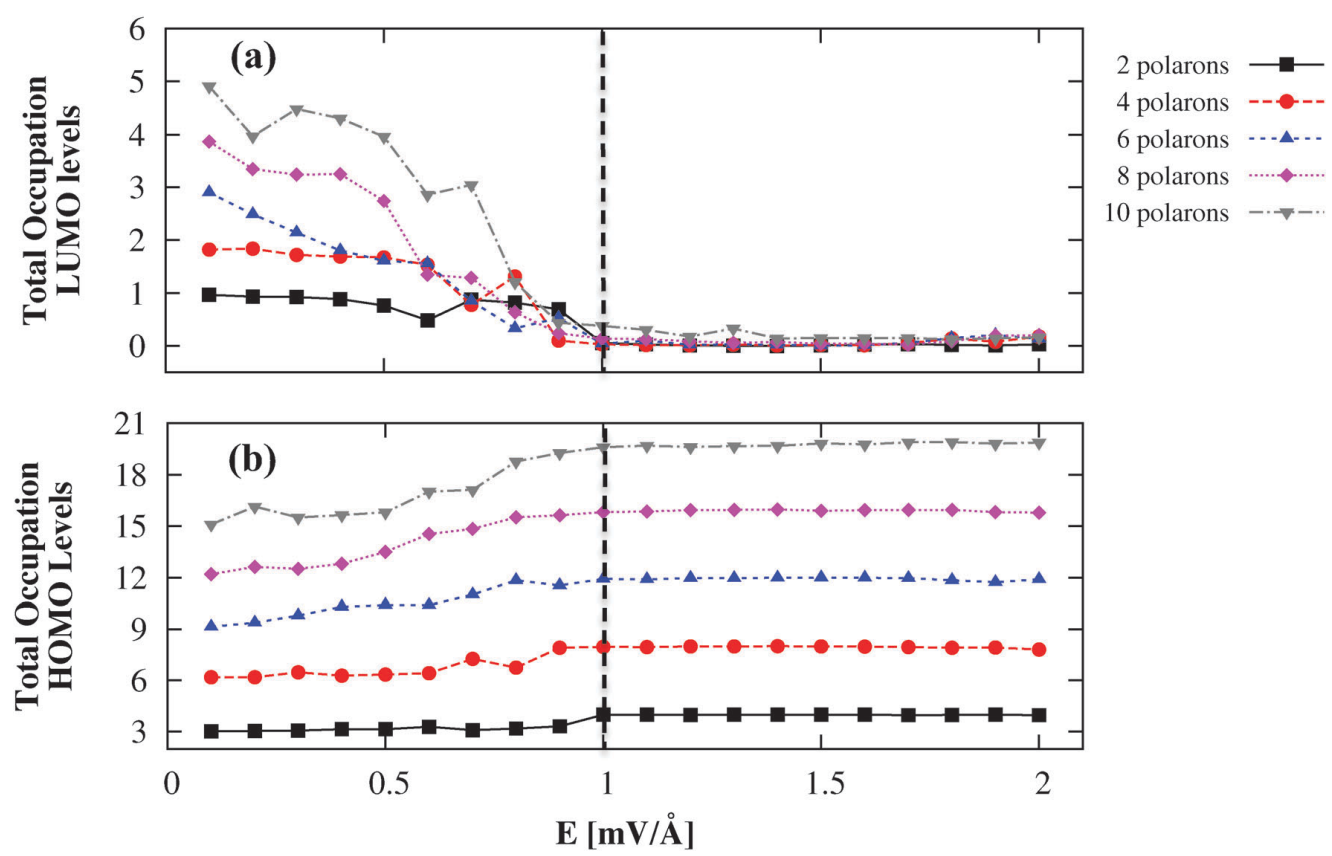

Fig. 7 Occupation numbers for the LUMO (a) and HOMO (b) levels for all the configurations of Fig. 6 with different electric field strengths. 
In other words, for electric fields higher than this critical value, no exciton nor polaron-excitons are present as the final state of the system. This is a consequence of the high energy that these electric field strengths impose on the system. The amount of energy is enough to dimerize the chain, thus yielding the neutral final state. As already discussed, for electric field strengths between 0 and $1.0 \mathrm{mV} \AA^{-1}$, a mixed final state between excitons and polaron-excitons is achieved. The relation between the HOMO and the LUMO also allows one to confirm the aforementioned hypothesis that with higher the concentrations, more polaron-excitons are formed compared to neutral excitations. This can be confirmed by the evaluation of the total HOMO/ LUMO ratio which shows that intermediate occupation is dominant for higher concentrations. This, again, is in accordance with the polaron-exciton presence picture. Therefore, the neutral excitation to polaron-exciton ratio depends directly on the final quantity of electrons in the LUMO (in relation to the HOMO). This dependence is observed to be a function of the initial charge carrier concentration. In the studies reported by An et $a l^{14}$ and Lie $e t a l .{ }^{16}$ the yield of the possible products formed from the recombination process between two oppositely charged polarons has been reported. An and coworkers have shown that the yield of the neutral excitation firstly increases with the electric field strength, reaching its maximum value $(\sim 47 \%)$ for $0.6 \mathrm{mV} \AA^{-1} .{ }^{14}$ For field strengths higher than this critical value, the yield for the neutral exciton decreases reaching $(\sim 35 \%)$ $1.0 \mathrm{mV} \AA^{-1}$. Lei and collaborators have reported that the total yield of excitons can reach at most $33.5 \%$ for an electric field strength of $0.5 \mathrm{mV} \AA^{-1}$, which indicates that it is not a complete annihilation of the polaron pair. ${ }^{16}$ Moreover, their results have shown that, for a collision of spin antiparallel polarons, the yield of singlet excitons reaches the maximum. In such case, the yields of singlet and triplet excitons are the same, and each is about $16.75 \%$. Just for the sake of information, very recently our previous research has shown that the presence of the temperature effects may increase the yield of neutral excitations to about $95 \%$, considering a temperature regime ranging from 50 to $160 \mathrm{~K}$ for a field strength of $1.5 \mathrm{mV} \AA^{-1}{ }^{18}$ These results give us a good insight into the products formed after the recombination dynamics between a polaron pair for different concentrations of charge carriers. We can conclude that, for electric field strengths smaller than $0.6 \mathrm{mV} \AA^{-1}$, the content of the products formed after the collision process is dominated by neutral excitations or quasi-neutral excitations. On the other hand, for electric field strengths ranging from 0.6 to $1.2 \mathrm{mV}^{-1}$, the content of the products is dominated by polaron-excitons. It is not difficult to conclude that for field strengths higher than $1.2 \mathrm{mV} \AA^{-1}$, the yield for the ground state reaches its maximum value $(\sim 100 \%)$, once that all the electrons have returned to the valence band, as shown in Fig. 7.

\section{Conclusions}

In this work we investigated the influence of different charge carrier concentrations and electric field strengths on the recombination dynamics between oppositely charged polarons. We were able to determine different regimes in which polaronexcitons or neutral excitations were mainly formed from the collisional process of different numbers of polarons. These regimes were observed to be dependent on both the electric field strength and on the concentration of the initial charge carriers. Moreover, it was found that, the higher the concentration of charge carriers the more probable it is to the collisional process to yield polaron-excitons rather than excitons. Furthermore, higher values of electric field also favor the formation of polaron-excitons until the critical value of $1.0 \mathrm{mV}^{-1}$, above which a dimerized chain is achieved. As actual electronic devices present high concentration of charge carriers, this study is of fundamental importance to the understanding of the particular conditions in which the desired kind of quasiparticle is to be formed. Therefore this kind of knowledge is important to improve the performance of organic photovoltaic devices such as Polymer Light Emitting Diodes.

\section{Acknowledgements}

The authors gratefully acknowledge the Swedish Research Council (VR) for financial support, the Swedish National Infrastructure for Computing (SNIC), and the financial support from the Brazilian Research Councils CNPq, CAPES and FINATEC.

\section{References}

1 Y.-H. Kim, T.-H. Han, H. Cho, S.-Y. Min, C.-L. Lee and T.-W. Lee, Polyethylene imine as an ideal interlayer for highly efficient inverted polymer light-emitting diodes, Adv. Funct. Mater., 2014, 24, 3808-3814.

2 K. Vandewal, S. Albrecht, E. T. Hoke, K. R. Graham, J. Widmer, J. D. Douglas, M. Schubert, W. R. Mateker, J. T. Bloking, G. F. Burkhard, A. Sellinger, J. M. J. Fréchet, A. Amassian, M. K. Riede, M. D. McGehee, D. Neher and A. Salleo, Efficient charge generation by relaxed charge-transfer states at organic interfaces, Nat. Mater., 2014, 13, 63-68.

3 Z. Sun, D. Liu, S. Stafström and Z. An, Scattering process between polaron and exciton in conjugated polymers, J. Chem. Phys., 2011, 134, 044906.

4 Z. Sun, Y. Li, K. Gao, D. S. Liu, Z. An and S. J. Xie, Dynamical study of polaron-bipolaron scattering in conjugated polymers, Org. Electron., 2010, 11, 279-284.

5 L. A. Ribeiro, P. H. O. Neto, W. F. da Cunha, L. F. Roncaratti, R. Gargano, D. A. da Silva Filho and G. M. e Silva, Exciton dissociation and charge carrier recombination processes in organic semiconductors, J. Chem. Phys., 2011, 135, 224901.

6 L. A. Ribeiro, W. F. da Cunha, P. H. O. Neto, R. Gargano and G. M. e Silva, Dynamical study of impurity effects on bipolaronbipolaron and bipolaron-polaron scattering in conjugated polymers, J. Phys. Chem. B, 2013, 117, 11801-11811.

7 B. Di, Y. Meng, Y. D. Wang, X. J. Liu and Z. An, Formation and evolution dynamics of bipolarons in conjugated polymers, J. Phys. Chem. B, 2011, 115, 964-971. 
8 Y. Meng, B. Di, X. J. Liu, Z. An and C. Q. Wu, Interchain coupling effects on dynamics of photoexcitations in conjugated polymers, J. Chem. Phys., 2008, 128, 184903.

9 Y. Meng, X. J. Liu, B. Di and Z. An, Recombination of polaron and exciton in conjugated polymers, J. Chem. Phys., 2009, 131, 244502.

10 L. A. R. Junior, W. F. da Cunha and G. M. e Silva, Temperature effects on the scattering of polarons and bipolarons on organic conductors, J. Phys. Chem. A, 2014, 118, 6272-6277.

11 L. A. R. Junior, W. F. da Cunha and G. M. e Silva, Temperature effects on intrachain recombination of bipolarons in conjugated polymers, Chem. Phys. Lett., 2014, 614, 151-155.

12 W. F. da Cunha, L. A. R. Junior, A. L. de Almeida Fonseca, R. Gargano and G. M. e Silva, Reactive scattering between excitons and charge carriers in conjugated polymers, J. Phys. Chem. C, 2014, 118, 23451-23458.

13 Z. Sun and S. Stafström, Spin-dependent polaron recombination in conjugated polymers, J. Chem. Phys., 2012, 136, 244901.

14 Z. An, B. Di and C. Q. Wu, Inelastic scattering of oppositely charged polarons in conjugated polymers, Eur. Phys. J. B, 2008, 63, 71-77.

15 Y. Li, K. Gao, Z. Sun, S. Yin, D.-S. Liu and S.-J. Xie, Intrachain polaron motion and geminate combination in donoracceptor copolymers: Effects of level offset and interfacial coupling, Phys. Rev. B: Condens. Matter Mater. Phys., 2008, 78, 014304.

16 J. Lei, Z. Sun, Y. Zhang and S. Xie, Effect of spin polarization on exciton formation in conjugated polymers, Org. Electron., 2011, 10, 1489-1494.

17 L. A. Ribeiro, W. F. da Cunha, P. H. O. Neto, R. Gargano and G. M. e Silva, Impurity effects on polaron-exciton formation in conjugated polymers, J. Chem. Phys., 2013, 139, 174903.

18 W. F. da Cunha, L. A. R. Junior, R. Gargano and G. M. e Silva, Critical temperature and products of intrachain polaron recombination in conjugated polymers, Phys. Chem. Chem. Phys., 2014, 16, 17072-17080.

19 W. P. Su, J. R. Schrieffer and A. J. Heeger, Solitons in polyacetylene, Phys. Rev. Lett., 1979, 42, 1698-1701.

20 W. P. Su, J. R. Schrieffer and A. J. Heeger, Solitons excitations in polyeacetylene, Phys. Rev. B: Condens. Matter Mater. Phys., 1980, 22, 2099-2111.

21 S. Brazovskii and N. Kirova, Physical theory of excitons in conducting polymers, Chem. Soc. Rev., 2010, 39, 2453-2465.

22 S. Stafström, Electron localization and the transition from adiabatic to nonadiabatic charge transport in organic conductors, Chem. Soc. Rev., 2010, 39, 2484-2499.

23 Z. G. Yu, M. W. Wu, X. S. Rao and A. R. Bishop, Excitons in two coupled conjugated polymer chains, J. Phys.: Condens. Matter, 1996, 8, 8847-8857.

24 L. A. Ribeiro, W. F. da Cunha, P. H. O. Neto, R. Gargano and G. M. e Silva, Impurity effects and temperature influence on the exciton dissociation dynamics in conjugated polymers, Chem. Phys. Lett., 2013, 580, 108-114.

25 L. A. Ribeiro, W. F. da Cunha, P. H. O. Neto, R. Gargano and G. M. e Silva, Effects of temperature and electric field induced phase transitions on the dynamics of polarons and bipolarons, New J. Chem., 2013, 37, 2829-2836.

26 L. A. Ribeiro, W. F. da Cunha, P. H. O. Neto and G. M. e Silva, Dynamics of photogenerated polaron-excitons in organic semiconductors, Phys. Procedia, 2012, 28, 112-116.

27 A. Johansson and S. Stafström, Polaron dynamics in a system of coupled conjugated polymer chains, Phys. Rev. Lett., 2001, 86, 3602-3605.

28 A. Johansson and S. Stafström, Nonadiabatic simulations of polaron dynamics, Phys. Rev. B: Condens. Matter Mater. Phys., 2004, 69, 235205.

29 Z. An, C. Q. Wu and X. Sun, Dynamics of photogenerated polarons in conjugated polymers, Phys. Rev. Lett., 2004, 93, 216407.

30 L. A. Ribeiro, W. F. da Cunha, P. H. O. Neto, R. Gargano and G. M. e Silva, Predicting the equilibrium structure of organic semiconductors with genetic algorithms, Chem. Phys. Lett., 2013, 555, 168-172.

31 G. M. e Silva, Electric-field effects on the competition between polarons and bipolarons in conjugated polymers, Phys. Rev. B: Condens. Matter Mater. Phys., 2000, 61, 10777-10781.

32 M. P. Lima and G. M. e Silva, Dynamical evolution of polaron to bipolaron in conjugated polymers, Phys. Rev. B: Condens. Matter Mater. Phys., 2006, 74, 224303.

33 H. Zhao, Y.-G. Chen, X.-M. Zhang, Z. An and C.-Q. Wu, Correlation effects on the dynamics of bipolarons in nondegenerate conjugated polymers, J. Chem. Phys., 2009, 130, 234908.

34 Y. Meng, B. Di, X. J. Liu, Z. An and C. Q. Wu, Effects of e-e interactions on the dynamics of polarons in conjugated polymers, Europhys. Lett., 2007, 79, 17002-17005.

35 H. Ma and U. Schollwöck, Dynamical simulations of charged soliton transport in conjugated polymers with the inclusion of electron-electron interactions, J. Chem. Phys., 2008, 129, 244705.

36 H. Ma and U. Schollwöck, Effect of electron-electron interactions on the charge carrier transitions in transpolyacetylene, J. Phys. Chem. A, 2010, 114, 5439-5444.

37 H. Ma and U. Schollwöck, Dynamical simulations of polaron transport in conjugated polymers with the inclusion of electronelectron interactions, J. Phys. Chem. A, 2009, 113, 1360-1367.

38 Y. L. Zhang, X. J. Liu, Z. Sun and Z. An, Dynamics of exciton transfer in coupled polymer chains, J. Chem. Phys., 2013, 138, 174906.

39 Y. L. Zhang, X. J. Liu, Z. Sun and Z. An, Temperature effects on the dynamics of photoexcitations in conjugated polymers, J. Phys. Chem. C, 2014, 118, 2963-2969.

40 D. Moses, H. Okumoto, C. H. Lee, A. J. Heeger, T. Ohnishi and T. Noguchi, Mechanism of carrier generation in poly(phenylene vinylene): Transient photoconductivity and photoluminescence at high electric fields, Phys. Rev. B: Condens. Matter Mater. Phys., 1996, 54, 4748-4754.

41 Y. Yao, Y. Qiu and C. Q. Wu, Dissipative dynamics of charged polarons in organic molecules, J. Phys.: Condens. Matter, 2011, 23, 305401.

42 Z. Sun and S. Stafström, Dynamics of exciton dissociation in donor-acceptor polymer heterojunctions, J. Chem. Phys., 2013, 138, 164905. 


\title{
Appegiox $M$ PAPER XIII
}

\section{Transport of Polarons in Graphene Nanoribbons}

\author{
Luiz Antonio Ribeiro Junior \\ Wiliam Ferreira da Cunha \\ Antonio Luciano de Almeida Fonseca \\ Geraldo Magela e Silva \\ Sven Stafström
}

J. Phys. Chem. Lett., 2015, 6, 510-514. 
PHYSICAL CHEMISTRY

\title{
Transport of Polarons in Graphene Nanoribbons
}

\author{
Luiz Antonio Ribeiro, Jr., ${ }^{\dagger \dagger}$ Wiliam Ferreira da Cunha, ${ }^{*}, \stackrel{*}{*}$ Antonio Luciano de Almeida Fonseca, ${ }^{+}$ \\ Geraldo Magela e Silva, ${ }^{*}$ and Sven Stafström
}

${ }^{\dagger}$ Department of Physics, Chemistry and Biology (IFM), Linköping University, SE-581 83 Linköping, Sweden

${ }^{\ddagger}$ Institute of Physics, University of Brasilia, 70.919-970 Brasilia, Brazil

S Supporting Information

ABSTRACT: The field-induced dynamics of polarons in armchair graphene nanoribbons (GNRs) is theoretically investigated in the framework of a twodimensional tight-binding model with lattice relaxation. Our findings show that the semiconductor behavior, fundamental to polaron transport to take place, depends upon of a suitable balance between the GNR width and the electronphonon (e-ph) coupling strength. In a similar way, we found that the parameter space for which the polaron is dynamically stable is limited to an even narrower region of the GNR width and the $\mathrm{e}-\mathrm{ph}$ coupling strength. Interestingly, the interplay between the external electric field and the e-ph coupling plays the role to define a phase transition from subsonic to supersonic velocities for polarons in GNRs.

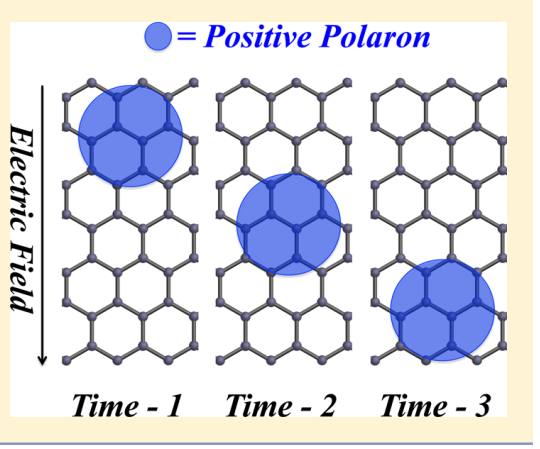

G raphene-based materials ${ }^{1-4}$ have emerged in the past few years as promising solutions to the design of a novel class of electronic devices. This carbon-based technology is expected to present greater efficiency, lower cost, as well as smaller environmental impact when compared to its inorganic counterpart for photovoltaic ${ }^{5}$ and energy storage ${ }^{6}$ applications. The strongly covalently bound two-dimensional structure of graphene results in unique properties such as high thermal and electrical conductivities, excellent mechanical strength, and reasonable transparency. Graphene nanoribbons (GNRs) are a special kind of structure derived from specific cuts of a graphene sheet. Besides sharing several of the interesting properties of the two-dimensional original material system, GNRs have, depending on the system's symmetry, the possibility to present a finite band gap. This feature is of crucial importance for electronics applications. Altogether, this makes GNRs of large interest with regards to both fundamental aspects, such as the basic mechanisms behind charge transport, and more applied studies related to device properties.

A great deal of theoretical ${ }^{7}$ and experimental ${ }^{8}$ efforts have been devoted to understanding the properties involved in the charge-transport mechanism in GNRs in order to enhance the performance of graphene-based optoelectronic devices. Some relevant studies have investigated the metallic-like behavior of the electronic transport in GNRs as a function of electric field, ${ }^{9}$ temperature, ${ }^{10}$ doping, ${ }^{11}$ variable widths, and different crystallographic orientations. ${ }^{12}$ Nonetheless, investigations regarding the semiconducting-like behavior and the possibility that the charge transport can be described by quasi-particles are still incipient, and further investigations that address the necessary conditions for stability and mobility of quasi-particles in GNRs are required.
It is well-established that low-dimensional carbon-based conductors, in particular, the one-dimensional polymeric system, ${ }^{13}$ present the novel property that its lattice structure can be locally distorted to form self-trapped charged states such as polarons. Moreover, it is known from studies of molecular crystals that both electron-lattice interactions and the system dimensionality are of fundamental importance to define the polaron characteristics. ${ }^{14}$ The charge associated with the polaron is distributed over the local deformation. It was both theoretically ${ }^{15}$ and experimentally ${ }^{16}$ reported that the charge carriers in GNRs are formed by more than one nonlocal lattice structure, which are symmetric regarding the charge density distribution and the lattice distortion degree. Interestingly, this structure presents a collective behavior for the charge transport in response of the applied electric field. Because all of these properties are common to regular polarons of other types of carbon-based conductors, we will throughout this work refer to these quasi-particles as polarons.

In this work, the transport of polarons in armchair GNRs is theoretically investigated in the framework of a two-dimensional tight-binding model in which the electron transfer (or hopping) integral includes the electron-phonon (e-ph) coupling as well as the force originating from an external electric field. Ehrenfest-like electron-lattice dynamics simulations were carried out in order to investigate the polaron behavior under different conditions of electric fields, GNR widths, and $\mathrm{e}-\mathrm{ph}$ coupling strengths. We chose this methodology due to its ease of implementation as well as satisfactory

Received: November 21, 2014

Accepted: January 22, 2015 
accuracy. It should be noted that, in this Letter, we are solely concerned with adiabatic processes. When considering the important problem of nonadiabatic transport, more sophisticated dynamical methodologies, such as surface hopping methods, should also be considered. ${ }^{17-19}$

It is the goal of this work to give a physical picture of the polarons' transport considering the interplay between the $\mathrm{e}-\mathrm{ph}$ coupling strength and the GNR width as well as the kinematic regime that these properties impose on the carriers.

In order to investigate the transport of polarons in GNRs, we have developed a two-dimensional generalization of a SSH-type Hamiltonian, ${ }^{13}$ modified to include an external electric field

$$
H=-\sum_{\langle i, j\rangle, s}\left(t_{i, j} C_{i, s}^{\dagger} C_{j, s}+\text { h.c. }\right)+\sum_{\langle i, j\rangle} \frac{K}{2} y_{i, j}^{2}+\sum_{i} \frac{p_{i}^{2}}{2 M}
$$

where $\langle i, j\rangle$ denotes summing over nearest-neighbor sites $i$ and $j$. For the electronic part, the operator $C_{i, s}^{\dagger}\left(C_{i, s}\right)$ creates (annihilates) a $\pi$-electron with spin $s$ at the ith site. $t_{i, j}=$ $\exp [-\mathrm{i} \gamma A(t) \delta]\left(t_{0}-\alpha y_{i, j}\right)$ is the hopping integral, where $t_{0}$ denotes the hopping of a $\pi$-electron between neighboring sites with no field present in an evenly spaced lattice, $\alpha$ is the $\mathrm{e}-\mathrm{ph}$ coupling constant, and $y_{i, j}$ is the relative displacement coordinate between neighboring sites. The electric field $\mathbf{E}(t)$, which was turned on adiabatically according to ref 20 in order to avoid numerical errors, is included in our model by means of the time-dependent vector potential $\mathbf{A}(t)$ through a Peierls substitution of the phase factor to the hopping integral. ${ }^{21}$ This is the simplest way of implementing electric fields consistently with periodic boundary conditions. $\gamma \equiv e a /(\hbar c)$, with $a$ being the lattice parameter, $e$ the absolute value of the electronic charge, and $c$ the speed of light. Depending on the bond type (BT) considered, as shown in Figure $1, \delta$ is 0.5 for BT-u, -0.5

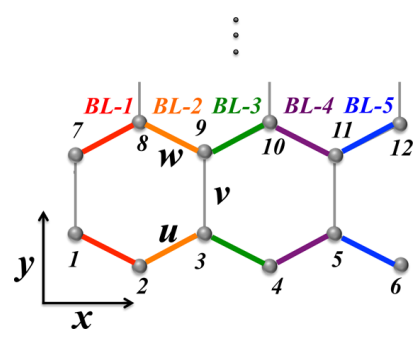

Figure 1. Schematic diagram representing the three different BTs considered in the expression for the vector potential vector. According to the convention adopted here, the atom index increases from the left to the right in the $x$-direction and upward in the $y$-direction.

for BT-w, and 1.0 for BT-v. The equation $\mathbf{E}(t)=-(1 / c) \dot{\mathbf{A}}(t)$ establishes the relation between the time-dependent vector potential and the electric field. The lattice backbone is described by the conjugated momentum $p_{i, j}$ to the displacement coordinate of each site, the harmonic force constant $K$, and the mass of a carbon atom $M$.

The lattice backbone dynamics is treated with a classical approach by means of a Newtonian equation $M \ddot{u}_{n}=F_{n}(t)$. Here, $F_{n}(t)$ represents the force experienced by a particular carbon atom $n$. The force expression is analogous to that originally developed by Silva and co-workers. ${ }^{15}$ Moreover, this equation can be numerically integrated according the methodology described in ref 22 . The electron dynamics is obtained by solving the time-dependent Schrödinger equation (TDSE). The wave functions are constructed by means of a linear combination of instantaneous eigenstates. Thus, the solutions of the TDSE can be expressed as

$$
\psi_{k, i}(t+\mathrm{d} t)=\sum_{l}\left[\sum_{m} \phi_{l, m}^{*}(t) \psi_{k, m}(t)\right] \mathrm{e}^{\left(-\mathrm{i} \varepsilon_{l} \mathrm{~d} t / \hbar\right)} \phi_{l, i}(t)
$$

in which $\left\{\phi_{l, i}(t)\right\}$ and $\left\{\varepsilon_{l}\right\}$ are the eigenfunctions and the eigenvalues of the Hamiltonian at a given time $t$, respectively. ${ }^{22}$ In this work, we have adopted the following values for the constants, $t_{0}=2.7 \mathrm{eV}$ and $K=57 \mathrm{eV} / \AA^{2}{ }^{21}$ The $\mathrm{e}-\mathrm{ph}$ coupling $\alpha$ is in the range from 3.5 to $10 \mathrm{eV} / \AA$, which according to the literature corresponds to suitable values for GNRs. ${ }^{23}$ This range for $\alpha$ is in very good agreement with values obtained in experimental studies for graphene, which are $6.0 \mathrm{eV} / \AA$, if the $\mathrm{e}-\mathrm{ph}$ coupling mechanisms other than the bond stretching can be neglected. ${ }^{23,24}$ Moreover, the energy band gaps obtained in our study (see the Supporting Information) are also in agreement with the band gap energies for GNRs obtained through electronic structure calculations. ${ }^{25,26}$

We report here results concerning armchair GNRs of 64 sites length $(90 \AA)$ and with periodic boundary conditions. This length exceeds by far typical polaron extensions and allows us to follow the polaron motion over relatively long time periods. Because one of the main goals of the present work is to investigate effects of different GNR widths on the transport mechanism, we considered several $64 \times n$ structures, where $n=$ 4-10 sites.

As polarons are structures that arise from the coupling between the charge of the system and the lattice distortion, it is crucial to accurately define this distortion through an order parameter. In order to do so, we display below the polaron in terms of both its charge distribution and the lattice distortion. The latter is defined by the difference in the carbon-carbon cond length $(\mathrm{BL})$ with respect to the $\mathrm{BL}$ of the pristine GNR. Figure 1 shows the five possible BLs from which the order parameter is constructed. Note that BL1 (BL2) is equivalent to BL5 (BL4).

As a starting point of our investigations, we have studied the behavior of the band gap value as a function of the $\mathrm{e}-\mathrm{ph}$ constant and the GNR width. The results are presented in the Supporting Information and are in very good agreement with earlier studies, which justifies the method used in this work. In the following, we restrict our studies of the features of the polarons to the parameter space that gives finite band gaps. In particular, from the two possible families of GNR widths, that is, $2 p$ and $2 p+1,9,12$ we limit the presentation below to the $2 p$ family $(n=4,6$, and 8$)$ because the band gap of the $2 p+1$ family is essentially zero and no polaron solution is possible.

The results presented in Figure 2 were obtained by varying $\alpha$ from 3.5 to $10 \mathrm{eV} / \AA$ with a step of $0.1 \mathrm{eV} / \AA$ for each nanoribbon. This range of $\alpha$ values includes the value of 6.0 $\mathrm{eV} / \AA$ obtained by Yan et al. ${ }^{23}$ The main information presented in this figure is the combination of widths and the values of $\alpha$ that can give rise to a charge localization corresponding to a stable polaron. The atoms in the nanoribbon are labeled according to Figure 1. The gray scale is a measure of charge localization in each site of the nanoribbon (displaced on the horizontal axis) as a function of the $\mathrm{e}-\mathrm{ph}$ coupling constant (vertical axis). The higher the charge localization, the darker the pattern.

Two interesting results stand out. The first, as already mentioned above, is the absence of a stable polaron solution for the $2 p+1$ family of GNRs. In other words, only representatives 


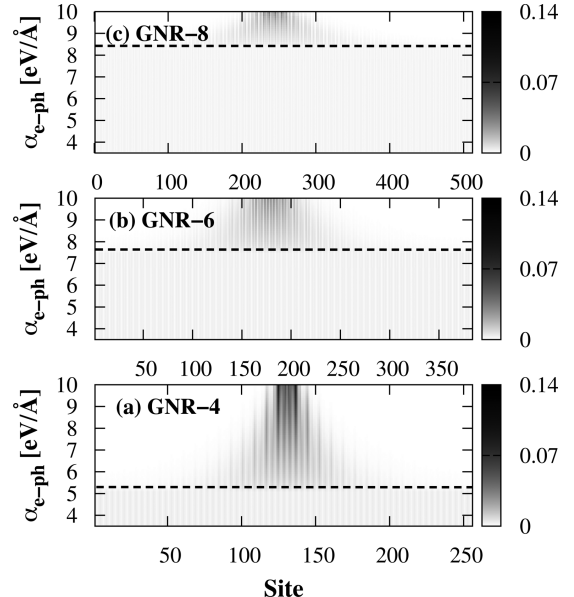

Figure 2. Charge density in the GNRs as a function of the e-ph coupling constant and for different GNRs: (a) $n=4$, (b) $n=6$, and (c) $n=8$.

of even families presented charge localization. The second important result is that the smaller the nanoribbon width, the higher the charge localization. This result reflects of the expected tendency of large nanoribbons to reach graphene in which no stable polaron solution exists. Overall, we have observed that the following critical values of $\alpha$ were needed in order to observe stable polarons, considering different GNR widths: $5.2 \mathrm{eV} / \AA$ for $n=4 ; 7.6 \mathrm{eV} / \AA$ for $n=6$; and $8.5 \mathrm{eV} / \AA$ for $n=8$. Theoretically, it is possible to obtain a polaron solution above $n=8$, but this requires a value of $\alpha$ that is far outside of the regime of physically reasonable values. Moreover, from Figure 2, it is possible to conclude for which regimes the charge-transport mechanism can be described as band-like transport. Considering $\mathrm{e}-\mathrm{ph}$ coupling strengths below the dashed line, it is clear that the charge is completely delocalized through the system, that is, there is no structure to mediate the charge transport in the physical picture of quasiparticles in semiconducting GNRs. It worth mentioning here that the phase transition from a critical polaron structure toward a completely delocalized state, depicted in Figure 2, is artificial because a certain GNR has one specific e-ph coupling strength value. In this way, this smooth transition indicates that, considering $\mathrm{e}-\mathrm{ph}$ coupling strengths higher than a critical value, the system has a semiconducting behavior and the charge transport can be mediated by a polaronic structure $(\mathrm{e}-\mathrm{ph}$ coupling values above the line).

We now turn to the kinetics of polarons under the influence of an external electric field. Figure 3 shows how the polaron's velocity is related to the $\mathrm{e}-\mathrm{ph}$ coupling for an electric field of $0.5 \mathrm{mV} / \AA$ (Figure 3a) and $2.0 \mathrm{mV} / \AA$ (Figure 3b) and for different GNR widths.

The onset of the polaron velocities naturally coincides with the different critical values of $\alpha$ needed to observe stable polarons (see above) and are not electric-field-dependent. A further increase in the $\mathrm{e}-\mathrm{ph}$ coupling strength induces larger lattice distortions and therefore a large polaron effective mass. This is the reason for the decaying velocities with increasing $\alpha$. Also, as should be expected, the higher electric field strength leads to an increase in the polaron velocities for a given value of $\alpha$. We can see that even the decreasing tendency of the carrier

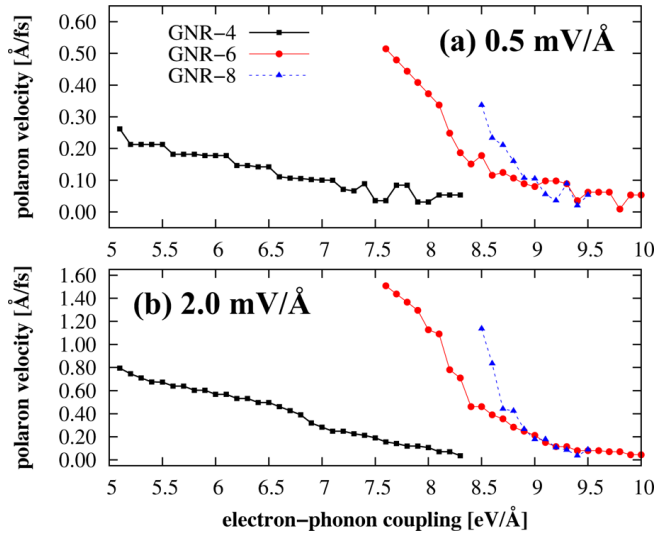

Figure 3. Polaron dynamics in GNRs of different widths subjected to (a) 0.5 and (b) $2.0 \mathrm{mV} / \AA$ electric fields.

velocity with $\alpha$ values, which is observed to vary among different widths, remains unaffected (i.e., varies in the same way) by different values of electric fields. Thus, the presence of polarons in the lattice is determined by a suitable balance between $\mathrm{e}-\mathrm{ph}$ and width, and therefore, the electric field plays no role in this matter.

A straightforward calculation carried out according to ref 27 shows that the sound velocity in an armchair GNR is approximately $0.3 \AA / \mathrm{fs}$. With this data at hand, we can analyze the velocity regime of the polarons and its relation to other properties of the system. It was found that for electric fields up to $0.5 \mathrm{mV} / \AA$, the $n=4$ nanoribbon presents charge carriers exclusively with subsonic velocities. As the nanoribbon size grows, it is possible to obtain supersonic regimes even for this smaller electric field. This is due to the delocalization observed in broader nanoribbons that tends to raise the polaron's mobility. Although $n=4$ presents a large occurrence of polarons for several values of $\mathrm{e}-\mathrm{ph}$ constant, their velocities are observed to be typically small. The $n=6$ nanoribbon, for instance, presents higher mobility of the polarons, particularly for $\alpha$ values between 7.4 and $8.1 \mathrm{eV} / \AA$. For $n=8$, the occurrence of fast polarons is intermediate, although it falls even faster with $\alpha$, and the main concentration of velocities is around low values. It should be noticed that these distributions can be inferred from the number of points (which corresponds to a given polaron) in each regime. As a summary, we observe an acoustic regime for polarons in $n=4$ and a varying, yet $\alpha$ dependent, distribution of acoustic and optical polarons for the other widths. The exception was for odd values of $n$ that do not present a polaron-mediated transport. Typically, we note that larger GNRs tend to present a higher incidence of polarons with larger velocities, but this behaviors exhibits some saturation effect for $n=8$.

Naturally, as we increased the electric field to $2.0 \mathrm{mV} / \AA$ (Figure $3 \mathrm{~b}$ ), even the $n=4$ nanoribbon presented supersonic components, and the occurrence of supersonic polarons increased for the other widths except for odd values of $n$, which were still free of polarons. One interesting feature that should be emphasized concerns the electric field values for which these supersonic regimes are observed in GNRs. It is known that for conjugated polymers, the critical electric field for a phase transition between the subsonic and supersonic regimes is on the order of $0.1 \mathrm{mV} / \AA^{28}$ On the other hand, we 
have found that for GNRs, no such transition took place for fields smaller than $0.5 \mathrm{mV} / \AA$ (and even greater for narrower GNRs). This is due to the very nature of the transport mechanism in these materials.

Conjugated polymers naturally present localized structures as charge carriers. In this case, the electric field is merely used to impose velocity on the system. GNRs, on the other hand, do not present such (almost) spontaneous localization. Therefore, the electric field is first necessary to yield some charge localization, generating the polaron that is following acceleration. Thus, the critical electric field to observe such a transition is, indeed, expected to be higher for GNRs compared to conjugated polymers. Another interesting remark concerns the velocities related to Figure 3. It is observed that when we compare their values to those observed in molecular systems such as a pentacene crystal, ${ }^{14}$ the obtained velocities are much greater than those on the molecular scale yet smaller than those on the atomic scale.

We now turn to results concerning the polaron dynamics in GNRs. Figure 4 shows the actual path of a polaron through an

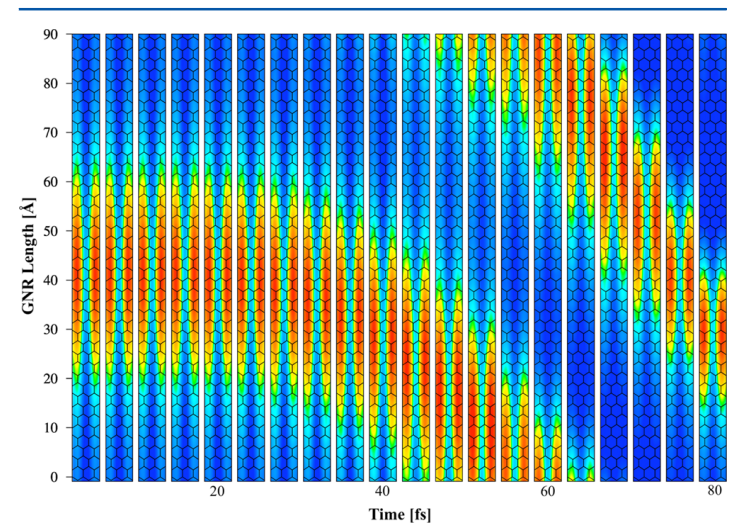

Figure 4. Polaron dynamics in an $n=6$ width armchair nanoribbon subjected to an electric field of $1.5 \mathrm{mV} / \AA ̊$.

armchair GNR with width $n=6$ and with periodic boundary conditions. The $\mathrm{e}-\mathrm{ph}$ coupling constant is set to $7.6 \mathrm{eV} / \AA$, and the external electric field is $1.5 \mathrm{mV} / \AA$, applied in the direction along the nanoribbon. It is important to note that the hexagonal grid presented in the figure is a mere representation of the GNR structure. Therefore, one should take the charge disposition over bondless regions of the ribbon as a qualitative pattern.

Two interesting properties stand out in the polarons dynamics of Figure 4. First is the delay in the polaron response to the applied electric field, and second is the difference between the initial and the steady-state extension of the polaron. Naturally, these two features are connected and have to do with the aforementioned two-fold role the electric field plays in polaron dynamics for GNRs. The electric field initially plays the role of assisting the charge localization in order to create a stable polaron; it is only after this task is accomplished that the collective behavior is fully manifested as the movement through the lattice. One can observe that as time goes by, a higher degree of localization is achieved. It is important to note that this is an expected feature derived from the quasi-particle gain of velocity through the electric field. Therefore, we can conclude that the applied electric field favors the polaron stability. It is worth mentioning that after $80 \mathrm{fs}$, the polaron achieves a supersonic regime of velocity, which is consistent with the discussion performed in Figure 3. For the cases shown in Figure 2, depending on the electric filed and e-ph coupling strengths for a certain GNR width, the time to arrive at constant polaron velocities ranges between 60 and 130 fs. In the case depicted in Figure 4, the polaron reaches its saturation velocity at about 80 fs.

Figure 5 presents the time evolution of the $\mathrm{BL}$ order parameter of the bonds shown in Figure 1 corresponding to the

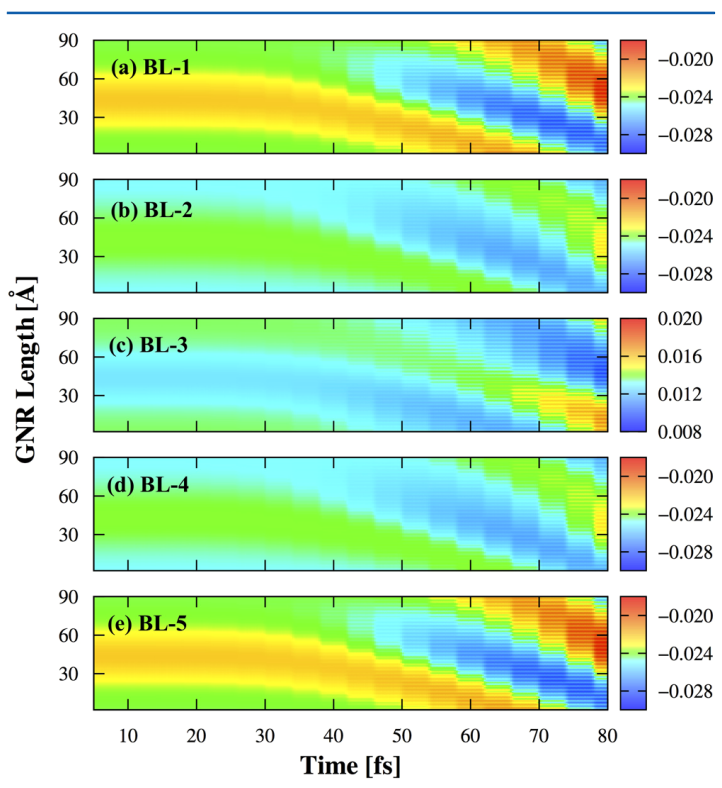

Figure 5. Time evolution of the lattice distortion for BTs from 1 (a) to 5 (e), according to what is defined in Figure 1.

charge density presented in Figure 4. Panels (a), (b), and (c) show the time evolution for BL1, BL2, and BL3, respectively. As mentioned above, it is obvious from Figure 1 that BL1 and BL5 are identical. The same holds for BL2 and BL4.

It is interesting to note that the bond lengths BL1, BL2, and BL3 decrease in the center of the polaron, just as in the case of conducting polymers. By comparing Figures 4 and 5 , we observe that the charge of the polaron is localized to the region of $\mathrm{BL} 1$ and $\mathrm{BL} 2$ and there is no charge in the central region around BL3. The presence of the charge results in a change in the bond order in such a way that a compression is favored. The expansion of BL3 is a compensation effect to the compression of $\mathrm{BL} 1$ and $\mathrm{BL} 2$. It is also clear that as the simulation time increases, the BLs' distortions become more and more localized. This is an already reported fact that has to do with the electric field role of promoting charge concentration in GNRs by providing velocity to the polaron.

An interesting fact easily noted from the figures is that not only the deformations associated with charge carriers but also those originating from the lattice vibrations increase with time. This can be evaluated by the blurring of the image that is a manifestation of the phonon normal modes. In other words, as the polaron travels trough the lattice, a considerable amount of phonon modes are generated by this movement. Although these phonons are usually spread over the whole lattice, it is 
possible to recognize some vibrations of large amplitudes, particularly toward the end of the simulation.

In summary, we have performed a systematic numerical study on charge carrier dynamics in armchair GNRs by means of a two-dimensional tight-binding model with lattice relaxation. By comparing different GNR widths and $\mathrm{e}-\mathrm{ph}$ constants, we could determine the critical conditions for a polaron-mediated transport to take place. Our methodology was able to accurately predict the band gap dependence on the width of the GNRs as well as their values (results are reported in the Supporting Information). A careful investigation on the velocity regimes of polarons was also performed. We observed that electric fields favor the occurrence of supersonic polarons. Although the electric field is not able to favor their formation, a role played by $\mathrm{e}-\mathrm{ph}$ coupling and width, a considerable gain in stability was implemented in the polarons. It was also observed that wider nanoribbons give rise to faster polarons. Their velocities were determined, and an investigation concerning their distribution was performed. Finally, the movement of polarons is followed by different phonons that can, in turn, interact with the polarons, thus affecting their dynamics.

\section{ASSOCIATED CONTENT}

\section{S Supporting Information}

Information about the energy gap calculations. This material is available free of charge via the Internet at http://pubs.acs.org.

\section{AUTHOR INFORMATION}

\section{Corresponding Authors}

*E-mail: luiju@ifm.liu.se (L.A.R.Jr.).

*E-mail: wiliam@fis.unb.br (W.F.d.C.).

Notes

The authors declare no competing financial interest.

\section{ACKNOWLEDGMENTS}

The authors gratefully acknowledge the Swedish Research Council (VR) for financial support, the Swedish National Infrastructure for Computing (SNIC), and the financial support from the Brazilian Research Councils CNPq, CAPES, and FINATEC

\section{REFERENCES}

(1) Novoselov, K. S.; Geim, A. K.; Morozov, S. V.; Jiang, D.; Zhang, Y.; Dubonos, S. V.; Grigorieva, I. V.; Firsov, A. A. Electric Field Effect in Atomically Thin Carbon Films. Science 2004, 306, 666-669.

(2) Stankovich, S.; Dikin, D. A.; Dommett, G. H. B.; Kohlhaas, K. M.; Zimney, E. J.; Stach, E. A.; Piner, R. D.; Nguyen, S. T.; Ruoff, R. S. Graphene-Based Composite Materials. Nat. Lett. 2006, 442, 282-286.

(3) Geim, A. K.; Novoselov, K. S. The Rise of Graphene. Nat. Mater. 2007, 6, 183-191.

(4) Li, X.; Wang, X.; Zhang, L.; Lee, S.; Dai, H. Chemically Derived, Ultrasmooth Graphene Nanoribbon Semiconductors. Science 2008, 319, 1229-1231.

(5) Yip, H.-L.; Jen, A. K. Y. Recent Advances in Solutions-Processed Interfacial Materials for Efficient and Stable Polymer Solar Cells. Energy Environ. Sci. 2012, 5, 5994-6011.

(6) Pumera, M. Graphene-Based Nanomaterials for Energy Storage. Energy Environ. Sci. 2011, 4, 668-674.

(7) Son, Y. W.; Cohen, M. L.; Louie, S. G. Half-Metalic Graphene Nanoribbons. Nat. Lett. 2006, 444, 347-342.

(8) Baringhaus, J.; Ruan, M.; Edler, F.; Tejeda, A.; Sicot, M.; TalebIbrahimi, A.; Li, A.-P.; Jiang, Z.; Conrad, E. H.; Berger, C.; et al. Exceptional Ballistic Transport in Epitaxial Graphene Nanoribbons. Nat. Lett. 2014, 506, 349-354.
(9) Mak, K. F.; Lui, C. H.; Shan, J.; Heinz, T. F. Observation of an Electric-Field-Induced Band Gap in Bilayer Graphene by Infrared Spectroscopy. Phys. Rev. Lett. 2009, 102, 256405-256409.

(10) Han, M. Y.; Brant, J. C.; Kim, P. Electron Transport in Disordered Graphene Nanoribbons. Phys. Rev. Lett. 2010, 104, 056801-056805.

(11) Martins, T. B.; Miwa, R. H.; da Silva, A. J. R.; Fazzio, A. Electronic and Transport Properties of Boron-Doped Graphene Nanoribbons. Phys. Rev. Lett. 2007, 98, 196803-196807.

(12) Han, M. Y.; Özyilmaz, B.; Zhang, Y.; Kim, P. Energy Band-Gap Engineering of Graphene Nanorribbons. Phys. Rev. Lett. 2007, 98, 206805-206809.

(13) Su, W. P.; Schrieffer, J. R.; Heeger, A. J. Solitons in Polyacetylene. Phys. Rev. Lett. 1979, 42, 1698-1701.

(14) Mozafari, E.; Stafström, S. Polaron Dynamics in a TwoDimensional Holstein-Peierls System. J. Chem. Phys. 2013, 138, 184104-184111.

(15) de Oliveira Neto, P. H.; Teixeira, J. F.; da Cunha, W. F.; Gargano, R; e Silva, G. M. Electron-Lattice Coupling in Armchair Graphene Nanoribbons. J. Phys. Chem. Lett. 2012, 3, 3039-3042.

(16) Yang, H.; Mayne, A. J.; Boucherit, M.; Comtet, G.; Dujardin, G.; Kuk, Y. Quantum Interference Channeling at Graphene Edges. Nano Lett. 2010, 10, 943-947.

(17) Wang, L.; Prezhdo, O. V. A Simple Solution to the Trivial Crossing Problem in Surface Hopping. J. Phys. Chem. Lett. 2014, 5, 713-719.

(18) Wang, L.; Beljonne, D. Flexible Surface Hopping Approach to Model the Crossover from Hopping to Band-Like Transport in Organic Crystals. J. Phys. Chem. Lett. 2013, 4, 1888-1894.

(19) Wang, L.; Trivedi, D.; Prezhdo, O. V. Global Flux Surface Hopping Approach for Mixed Quantum-Classical Dynamics. J. Chem. Theory Comput. 2014, 10, 3598-3605.

(20) Ribeiro, L. A.; da Cunha, W. F.; Neto, P. H. O.; Gargano, R.; e Silva, G. M. Effects of Temperature and Electric Field Induced Phase Transitions on the Dynamics of Polarons and Bipolarons. New J. Chem. 2013, 37, 2829-2836.

(21) Neto, A. H. C.; Guinea, F.; Peres, N. M. R.; Novoselov, K. S.; Geim, A. K. The Electronic Properties of Graphene. Rev. Mod. Phys. 2009, 81, 109-162.

(22) Lima, M. P.; e Silva, G. M. Dynamical Evolution of Polaron to Bipolaron in Conjugated Polymers. Phys. Rev. B 2006, 74, 224303224309.

(23) Yana, J.; Zhang, Y.; Golerb, S.; Kim, P.; Pinczuk, A. Raman Scattering and Tunable Electron-Phonon Coupling in Single Layer Graphene. Solid State Commun. 2007, 143, 39-43.

(24) Yan, J.; Zhang, Y.; Kim, P.; Pinczuk, A. Electric Field Tunin of Electron-Phonon Coupling in Graphene. Phys. Rev. Lett. 2007, 98, $166802-166806$

(25) Yang, L.; Park, C.-H.; Son, Y.-W.; Cohen, M. L.; Louie, S. G. Quasiparticle Energies and Band Gaps in Graphene Nanoribbons. Phys. Rev. Lett. 2007, 99, 186801-186805.

(26) Son, Y.-W.; Cohen, M. L.; Louie, S. G. Energy Gaps in Graphene Nanoribbons. Phys. Rev. Lett. 2006, 97, 216803-216807.

(27) Vos, F. L. J.; Alberts, D. P.; van Saarloos, W. Simple Method for Calculating the Speed of Sound in Tight-Binding Models: Application to the Su-Schrieffer-Heeger Model. Phys. Rev. B 1996, 53, 59865989(R).

(28) Johansson, A. A.; Stafström, S. Nonadiabatic Simulations of Polaron Dynamics. Phys. Rev. B 2004, 69, 235205-235211. 